\title{
Appalachian Caregivers of Persons with Dementia Non Use of Services
}

Kristina M. Childers

West Virginia University, ckchilz1@live.com

Follow this and additional works at: https://researchrepository.wvu.edu/etd

Part of the Nursing Commons

\section{Recommended Citation}

Childers, Kristina M., "Appalachian Caregivers of Persons with Dementia Non Use of Services" (2019). Graduate Theses, Dissertations, and Problem Reports. 3909.

https://researchrepository.wvu.edu/etd/3909

This Dissertation is protected by copyright and/or related rights. It has been brought to you by the The Research Repository @ WVU with permission from the rights-holder(s). You are free to use this Dissertation in any way that is permitted by the copyright and related rights legislation that applies to your use. For other uses you must obtain permission from the rights-holder(s) directly, unless additional rights are indicated by a Creative Commons license in the record and/ or on the work itself. This Dissertation has been accepted for inclusion in WVU Graduate Theses, Dissertations, and Problem Reports collection by an authorized administrator of The Research Repository @ WVU.

For more information, please contact researchrepository@mail.wvu.edu. 
Appalachian Family Caregivers' of Persons with Dementia Non-use of Services

\author{
Kristina M. Childers \\ Dissertation submitted to the \\ School of Nursing at West Virginia University \\ in partial fulfillment of the requirements for the degree of \\ $\mathrm{PhD}$ in \\ Nursing
}

Georgia L. Narsavage, PhD, APRN, FNAP, Chair

Barbara L. Nunley, PhD, RN

Marilyn Smith, PhD, PMHNP-BC, APRN

Eric Rankin, $\mathrm{PhD}$

Shirley M. Neitch, MD

Department of Nursing

\title{
Morgantown, West Virginia \\ 2019
}

Keywords: caregivers, dementia, Appalachian, rural, non-use of services

Copyright 2019 Kristina M. Childers 


\section{Abstract \\ Appalachian Caregivers of Persons with Dementia Nonuse of Services}

Kristina M. Childers

Background: Despite evidence indicating that supportive resources can improve the experience of caregivers (CG) and care recipients (CR), many underutilize available services or do not use services at all. Understanding the factors associated with service use, and barriers to using available resources is important for health care providers to improve caregiver and care recipient outcomes. To comprehensively address CG needs for support services, the factors that impact non-use of services must be identified in Appalachia. The purpose of this current study was to explain factors most strongly associated with caregivers' non-use of formal and informal services in Appalachia.

Methods: A correlational explanatory design using validated self-report surveys was used to explain the association of selected factors with Appalachian caregiver's non-use of formal and informal services. The design followed a substruction of Andersen's Behavioral Model for Health Services Research. The purposive sample of family caregiver/person with dementia (PWD) dyads was recruited from the Appalachian region, primarily West Virginia, via caregiver support groups, geriatric clinics, primary care clinics, contact lists from geriatric associations, county agencies on aging, home health agencies, and senior centers within Appalachia. Data were collected via an in-person or mailed survey that participants completed in writing. Data were analyzed using descriptive statistics and ANOVA tests, calculating for medium effect size, considering two to four independent variables (eight in total) from the Andersen model entered into each research question, using a sample of $43 \mathrm{CG} / \mathrm{CR}$ dyads.

Results: The results of the analyses were used to answer the four research questions:

1) What estimate of the variance in the non-use of services in Appalachia can be explained by predisposing characteristics of demographic variables, social variables, and (health and service) belief variables? Data support that the Social Provisions Scale (SPS) explained $8.3 \%$ of variance in the dependent variable Non-Use of Services (R Square 0.107 - 10.7\%; Adjusted R Square 0.83 $-8.3 \%)$.

2) What estimate of the variance in the non-use of services in Appalachia can be explained by enabling factors of financial and organizational resource variables? Data support that Caregiving Service Availability explained $11.7 \%$ of the variance for Non-Use of Services (R Square 0.14 14.1\%; Adjusted R Square 0.117 - 11.7\%).

3) What estimate of the variance in the non-use of services in Appalachia can be explained by the need factors, both perceived and evaluated needs variables? Evaluated Need, a calculated variable that included having a primary care provider, the health care provider recommending services for the person with dementia (PWD), added to Paid and Volunteer hours of help received per week, explained 7.9\% of the variance for Non-Use of Services (R Square 0.102 $10.2 \%$; Adjusted R Square $0.079-7.9 \%$ ).

4) What combination of predisposing, enabling, and need factors constitutes the strongest explanatory or associative model of non-use of services? The Non-use of services final regression analysis entering the three significant variables of the Social Provisions Scale, Caregiving Service Availability, and Evaluated Need explained $40 \%$ of non-use of services. A theory-based forced-entry logistic regression on a dichotomous variable, User/Non-User of 
services, found the same variables significant. Linear Regression provided more explanatory strength.

Implications: In order to reduce non-use of services, more caregiving support services need to be available for CGs of PWD, and health care providers' recommendation of the use of support services would be helpful for CGs of PWD. 


\section{Table of Contents}

Chapter 1

Background of the Problem $\quad 2$

Impact of Caregiver non-use of formal and informal services.

$\begin{array}{ll}\text { Appalachian Region and Culture } & 5\end{array}$

$\begin{array}{ll}\text { Significance of Caregiver Non-Use of Formal and Informal Services } & 7\end{array}$

$\begin{array}{lr}\text { Major Components of the Study } & 8\end{array}$

Purpose: The purpose of this study was to explain factors most strongly associated with caregivers' non-use of formal and informal services in Appalachia. $\quad 8$

$\begin{array}{lc}\text { Research Questions. } & 8\end{array}$

$\begin{array}{ll}\text { Definition of terms. } & 8\end{array}$

$\begin{array}{ll}\text { Method } & 11\end{array}$

$\begin{array}{ll}\text { Theoretical Rationale. } & 12\end{array}$

$\begin{array}{ll}\text { Summary } & 13\end{array}$

$\begin{array}{ll}\text { Chapter } 2 & 15\end{array}$

$\begin{array}{ll}\text { Overview and Literature Search Process } & 15\end{array}$

$\begin{array}{ll}\text { Dementia. } & 15\end{array}$

Types of Dementia. 16 
$\begin{array}{lr}\text { Stages of Dementia } & 18\end{array}$

$\begin{array}{ll}\text { Trajectory of dementia. } & 20\end{array}$

$\begin{array}{ll}\text { Impact of dementia on caregivers } & 22\end{array}$

$\begin{array}{ll}\text { Caregiving Challenges } & 23\end{array}$

$\begin{array}{lr}\text { Progressive Loss and Cost } & 24\end{array}$

Caregivers and Use of Services for Persons with Dementia

$\begin{array}{ll}\text { Andersen's Model of Health Service Use } & 28\end{array}$

Predisposing, enabling, and need characteristics related to outcomes. 28

$\begin{array}{ll}\text { Qualitative Studies. } & 29\end{array}$

$\begin{array}{ll}\text { Quantitative Studies. } & 32\end{array}$

Individual Enabling Characteristics Related to Outcomes. 35

$\begin{array}{ll}\text { Qualitative Studies. } & 35\end{array}$

$\begin{array}{ll}\text { Quantitative Studies. } & 36\end{array}$

Expanding the Social Provision Scale (SPS). 43

Individual Need Characteristics Related to Outcomes 45

$\begin{array}{ll}\text { Qualitative Studies. } & 45\end{array}$

$\begin{array}{ll}\text { Quantitative Studies. } & 46\end{array}$

$\begin{array}{ll}\text { Outcomes Use/ Non-Use of Formal and Informal Services } & 47\end{array}$

$\begin{array}{ll}\text { Qualitative Studies. } & 47\end{array}$

$\begin{array}{ll}\text { Quantitative Studies. } & 47\end{array}$ 
$\begin{array}{ll}\text { Research Design } & 52\end{array}$

Population and Sample Selection.

$\begin{array}{ll}\text { Inclusion Criteria. } & 54\end{array}$

$\begin{array}{ll}\text { Exclusion Criteria. } & 54\end{array}$

$\begin{array}{lc}\text { Instrumentation } & 55\end{array}$

$\begin{array}{ll}\text { Predisposing Measures } & 55\end{array}$

Demographics and Social Variables.

Beliefs about Health (Dementia) Variable. $\quad 56$

Beliefs about Service (Dementia) Variable. $\quad 56$

$\begin{array}{ll}\text { Enabling Measures } & 57\end{array}$

$\begin{array}{ll}\text { Enabling Financing. } & 57\end{array}$

$\begin{array}{ll}\text { Enabling Organizing. } & 58\end{array}$

$\begin{array}{lr}\text { Need Measures } & 58\end{array}$

$\begin{array}{ll}\text { Perceived Need Variable. } & 58\end{array}$

Evaluated Need Variable. $\quad 59$

Non-Use of Services Measures $\quad 59$

Non-Use of Services (Dependent Variable). 59

The Final Survey/Questionnaire $\quad 60$ 
Data Analysis

$\begin{array}{lr}\text { Regression Analyses } & 69\end{array}$

$\begin{array}{ll}\text { Dependent Variables. } & 69\end{array}$

$\begin{array}{ll}\text { Predisposing Factors. } & 70\end{array}$

$\begin{array}{ll}\text { Enabling Factors. } & 72\end{array}$

$\begin{array}{ll}\text { Need Factors. } & 74\end{array}$

Combination of Predisposing, Enabling, and Need Factors Explaining the Model of Non-Use of Services. 
Overview of Problem. $\quad 80$

$\begin{array}{ll}\text { Review Purpose statement. } & 80\end{array}$

$\begin{array}{ll}\text { Review of the methodology. } & 81\end{array}$

$\begin{array}{ll}\text { Review Research Questions. } & 81\end{array}$

Review of Major Findings. $\quad 82$

Review of Additional Findings. $\quad 82$

$\begin{array}{ll}\text { Discussion } & 83\end{array}$

$\begin{array}{ll}\text { Predisposing. } & 83\end{array}$

Demographic and Social. $\quad 83$

Health Beliefs (Dementia) and Health Beliefs Services (Dementia). 84

$\begin{array}{ll}\text { Enabling. } & 85\end{array}$

$\begin{array}{ll}\text { Financial. } & 85\end{array}$

$\begin{array}{ll}\text { Organizing. } & 85\end{array}$

$\begin{array}{lr}\text { Need. } & 86\end{array}$

$\begin{array}{ll}\text { Evaluated. } & 86\end{array}$

$\begin{array}{ll}\text { Perceived. } & 87\end{array}$

$\begin{array}{ll}\text { Services Not Used. } & 88\end{array}$

$\begin{array}{lr}\text { Conclusions } & 89\end{array}$

Enabling Organizing Total Caregiving Services Available. 90

Opportunities for Nurses in Primary Care. $\quad 98$

Implications of Qualitative Data. $\quad 99$

$\begin{array}{ll}\text { Health Literacy. } & 100\end{array}$

$\begin{array}{ll}\text { Predisposing Social Support. } & 101\end{array}$ 
$\begin{array}{lr}\text { Limitations } & 102\end{array}$

$\begin{array}{ll}\text { Recruitment and Sample. } & 102\end{array}$

$\begin{array}{ll}\text { Surveys. } & 102\end{array}$

$\begin{array}{ll}\text { Recommendations } & 105\end{array}$

$\begin{array}{ll}\text { Lessons Learned. } & 106\end{array}$

$\begin{array}{lr}\text { References } & 108\end{array}$

Appendix A Abbreviations used throughout text of dissertation in alphabetical order 135

Appendix B Table 1: CG variables used in study Descriptive Statistics 138

Appendix C Figure 1: Andersen’s Behavioral Model of Service Use 154

Appendix C Figure 2: Andersen’s Behavioral Substructed Model of Service Use with variables from this Study (following data collection) 155

Appendix D Table 2 Inclusion and Exclusion Literature Search 156

Appendix E Table 3: Andersen's Model, Study Variables, and Measures Literature

$\begin{array}{ll}\text { Review and Survey } & 157\end{array}$

Appendix F Table 4. Expanded SPS and supporting literature $\quad 167$

Appendix F Social Provisions Scale-Short Version 177

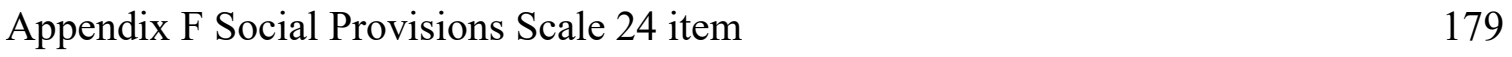

Appendix F Expanded Social Provisions Scale $\quad 182$

Appendix G Permission Letters for Measures 187

Appendix G Permission Letter Philadelphia Geriatric Center Multilevel Assessment

$\begin{array}{ll}\text { Instrument (PCG-MAI) } & 188\end{array}$ 
Appendix G Permission Letter Caregiver Mastery Subscale, part of the Caregiving Appraisal Scale

Appendix G Permission Letter Dr. Lyn Phillipson

Appendix G Letter of Permission Social Provisions Scale (SPS) Russell and Cutrona 2015

Appendix H Expanded Social Provisions Scale

Appendix I Research Survey

Appendix J Tables 6.1 Q1A - 6.12 Q4C Correlations and Linear Regressions from

Research Questions

Appendix J Table 6.2 Q1B Predisposing characteristic variables related to non-use of

formal services in Appalachia $(\mathrm{N}=40)$

Appendix J Table 6.3 Q1C Predisposing characteristic variables related to non-use of informal services in Appalachia $(\mathrm{N}=40)$

Appendix J Table 6.4 Q2A Enabling characteristic variables related to non-use of both services in Appalachia $(\mathrm{N}=38)$

Appendix J Table 6.5 Q2B Enabling characteristic variables related to non-use of formal services in Appalachia $(\mathrm{N}=38)$

Appendix J Table 6.6 Q2C Enabling characteristic variables related to non-use of informal services in Appalachia $(\mathrm{N}=38)$

Appendix J Table 6.7 Q3A Need characteristic variables related to non-use of both services in Appalachia $(\mathrm{N}=42)$

Appendix J Table 6.8 Q3B Need characteristic variables related to non-use of formal services in Appalachia $(\mathrm{N}=42)$ 
Appendix J Table 6.9 Q3C Need characteristic variables related to non-use of informal

services in Appalachia $(\mathrm{N}=42) \quad 228$

Appendix J Table 6.10 Q4A Strongest explanatory model of non-use of services both services $(\mathrm{N}=42)$

Appendix J Table 6.11 Q4B Strongest explanatory model of non-use of formal services

Appendix J Table 6.12 Q4C Strongest explanatory model of non-use of informal services

Appendix K Table 7 Inclusion and Exclusion Criteria for Study

Appendix L Map of Counties in WV and surrounding States with Respondent Counties

Identified

Appendix M All Questions Unmet Needs Assessment (UNA)

Appendix N Variables by Research Questions with Measures Table 8

Appendix P Family Caregiver Supports Included with Survey Packet

Appendix Q Revised Scales and Subscale Internal Consistencies and Reliabilities 


\section{Chapter 1}

Family CGs report both positive and negative outcomes of caring for a person with dementia. Positive outcomes include increased faith, a sense of reciprocity, as well as spiritual and personal growth (Brodaty \& Donkin, 2009). Negative consequences of caregiving involve emotional and physical distress, a reported poorer quality of life, guilt, social isolation, and financial burden (Brodaty \& Donkin, 2009). Despite evidence indicating that supportive resources can improve the experience of caregivers (CG) and care recipients (CR), many do not use, or underutilize, available services (Brodaty, Thomson, Thompson, \& Fine, 2005; Brown \& Chen, 2008; Robinson, Buckwalter, \& Reed, 2013; Wilks \& Croom, 2008). Understanding the factors associated with service use and barriers to using available resources are important to health care providers to improve caregiver and care recipient outcomes. Numerous caregiver outcomes are reported in the literature, both qualitatively and quantitatively. However, over the past 25 years, there has been a paucity of Appalachian caregiver literature. The most closely associated literature regarding caregivers of persons with dementia is related to rural caregivers, such as those in Appalachia. Research findings from studies of rural caregivers indicate the caregiving role is complicated by issues of access to care, transportation, affordability, adequacy, and awareness of services (Ervin \& Reed, 2015; Gerdner, Tripp-Reimer, \& Simpson, 2007; Goins, Spencer, \& Byrd, 2009; Kosberg, Kaufman, Burgio, Leeper, \& Sun, 2007; Morgan, Semchuk, Stewart, \& D’Arcy, 2002; Orpin, Stirling, Hetherington, \& Robinson, 2014). The purpose of this chapter is to explain why family caregiver use or non-use of available services in Appalachia is meaningful to nursing, the health care team, and public health workers, and provide support for the current study to add to the body of nursing knowledge. 
Appendix A includes a list of frequently used abbreviations in the study.

\section{Background of the Problem}

There may be many reasons why caregivers of persons with dementia do not use available formal (paid) or informal (unpaid) support services (Brodaty et al.,2005; O’Connell, Hawkins, Ostaszkiewicz, \& Millar, 2012; Phillipson, Jones, \& Magee, 2014). Caregiving requires energy, time, and commitment, and places the caregiver at increased risk for physical and psychological health problems. The use of formal and informal services, such as respite care, allows the caregiver intermittent relief from caregiving pressures, and can help to improve the overall health related quality of life of the caregiver and care recipient (Phillipson et al., 2014).

\section{Prevalence of Dementia}

Over five million Americans had Alzheimer's disease or related dementia (ADRD) in 2014 according to the Alzheimer's Association. Dementias cause progressive impairment in a person's ability to provide safe self-care, and the impairments eventually necessitate someone else assuming responsibility for supporting the PWD. Informal (unpaid) caregivers provided the majority of care to those affected by Alzheimer's and other dementias, contributing over 17 billion hours of care annually, with a current value of over two hundred billion dollars (Alzheimer's Association, 2014). Approximately 15 million Americans, typically spouses or adult children, provide this unpaid care. Caregiving tasks may include assistance with basic and instrumental activities of daily living (ADLs), including bathing, grooming, paying bills, shopping, transportation, managing medications, providing a safe environment, arranging or finding support services, and numerous other tasks (Alzheimer's Association, 2014). Family CGs of persons with dementia experience physical, psychological, social, and financial demands that may negatively affect their own health and quality of life (Alzheimer's Association, 2014). 
Complicating the problem in Appalachia, there are limited caregiver resources in rural regions (Goins et al., 2009; Kosberg et al., 2007; Thorpe, Van Houtven, Sleath, \& Thorpe, 2010). Also, the rural caregiver may be caring for another dependent. Kosberg et al. (2007) indicated that nearly one fifth of rural caregivers in their study in Alabama $(\mathrm{N}=28$ of 141) reported providing more than 30 hours of care per week to a second recipient. Additionally, lack of access to formal care providers has an impact on the cost of healthcare, and rates of hospitalizations. Care recipients with dementia living in rural areas had four times more hospital admissions than urban persons without dementia (Thorpe et al., 2010). Studies of caregivers of persons with dementia specifically living in Appalachia were not found.

One outcome of inadequate resources is that CGs have less access to accurate information about dementia and the needs of patients with the disorder. Information deficits include poor understanding of the etiology of dementia, with many rural caregivers crediting stress or suffering hardships in life as causing their own cognitive decline (Gerdner et al., 2007). Other rural caregivers attributed dementia decline to natural aging, or to a genetic predisposition (Gerdner et al., 2007). Obtaining an accurate diagnosis may be more difficult when resources are sparse. Morgan and colleagues (2002) found that rural caregiving families of persons with dementia reported that both health care providers and the family of the PWD appeared to have problems in recognizing dementia.

\section{Impact of Caregiver non-use of formal and informal services.}

Results from the study by Morgan et al., in 2002 identified a significant stigma associated with dementia as a major impediment to service use for rural caregivers. Seven other barriers to use of services for rural caregivers were the lack of privacy/anonymity; beliefs and attitudes (caregiver would be negatively viewed as unable to manage without help if he or she used a 
support service); lack of awareness (availability of support resources and importance of early use); financial barriers; acceptability of services; and challenges in service delivery (Morgan et al., 2002).

Caregiver declining health is also an escalating problem that would generally be expected to result in higher use of services, but which has not always led to this outcome in Appalachian CGs. Compared to non-caregivers, family caregivers had higher levels of stress hormones (23\%) and lower levels of antibody (15\%) responses (American Psychological Association, 2006). High levels of stress hormones can contribute to diabetes and heart disease, and lower antibody levels reduce the body's defense against infection, which can lead to poor physical health (American Pyschological Association, 2006). According to Reinhard, Given, Petlick, and Bemis (2008), there was a greater than $60 \%$ higher rate of death for spousal caregivers compared to noncaregivers of the same age. Caregivers were at risk for sleep impairment, fatigue, reduced immune function, higher insulin levels, elevated blood pressure, increased cardiovascular disease, symptoms of depression, greater levels of anxiety, compromised self-care such as exercise, and having a chance of using more prescription medications (Reinhard et al., 2008). Overwhelmed or fatigued caregivers place the care recipient at higher risk for elder abuse and medication errors. There was also an increased threat of family conflict with an exhausted caregiver (Reinhard et al., 2008). Not surprisingly, in the face of these health facts, Reinhard and colleagues found that $75 \%$ of caregivers of persons with dementia reported unmet needs, but less than $20 \%$ used any support services (9\% used respite services, $11 \%$ attended support groups). The researchers suggested a need to help caregivers navigate the service delivery system in identifying and using available resources (Reinhard et al., 2008). 
At least one of every two caregivers reported their emotional stress levels as being high or very high, and more than one in three suffered from depression (Alzheimer's Association, 2015a). According to the Alzheimer's Association (2015a) more than $66 \%$ of caregivers are women and over $34 \%$ of these are age 65 years or older. The physical and emotional toll on the caregivers' health resulted in over nine billion dollars in healthcare costs (hospitalizations and emergency room visits) in 2012 (Alzheimer's Association, 2014). Health care costs for PWD receiving Medicare resulted in almost $\$ 10,000$ in out-of-pocket expenses for patients and their families in 2013 (Alzheimer's Association, 2014). The cost of dementia care was higher than other chronic illness expenses, and the time required providing care was progressively longer. Consequently, assisting caregivers early in the caregiving process can improve their emotional and physical outcomes, and using formal and informal services may be one method to reduce health decline and health care expenses.

\section{Appalachian Region and Culture}

The Appalachian Regional Commission (ARC) identifies Appalachia as a geographic area following the Appalachian Mountains through 420 counties in 13 states, with West Virginia the only state entirely designated as “Appalachian" (Appalachian Regional Commission, 2018). There are sub regions of Appalachia: Northern (includes designated Appalachian counties in Maryland, New York, Ohio, and Pennsylvania), Central (includes designated Appalachian counties in Kentucky, some counties in Tennessee, some counties in Virginia, and West Virginia), and Southern Appalachia (designated Appalachian counties in Tennessee, Virginia, Alabama, Georgia, Mississippi, North Carolina, and South Carolina) (Cuoto, 2006). There are also large metropolitan areas near Appalachian designated counties, including Pittsburgh, Pennsylvania; Cincinnati, Ohio; Columbus, Ohio; Atlanta, Georgia; and Nashville, Tennessee. 
People in Appalachia often refer to themselves as "mountaineers" or mountain people" rather than as "Appalachian" (Russ, 2010). Russ posits that

“Appalachian culture is person oriented rather than task oriented, and a person's identity is dependent on their community and kinship ties. Since identity is dependent on the group, Appalachians avoid confrontation that might jeopardize their standing in the group and interfere with its smooth operation. Complaints and dissatisfaction are usually expressed indirectly" (2010, p. 2).

Culture itself is viewed as a way of life and thinking, and there are two contrasting lists of descriptors of Appalachian culture. McGarvey, Leon-Verdin, Killos, Guterbock, and Cohn (2011) describe the culture in Appalachia as having a strong belief in community, family support, and social ties; deep religious faith, strong work principles, being dependable, and a feeling of "belonging in the mountains" (p.348). Others have used more negative descriptors including having a resigned philosophy of life (fatalism), geographically isolated, distrusting of people from outside the local region, and suspicious of the organized health care systems (McGarvey et al., 2011; Rosswurm, Dent, Armstrong-Persily, Woodburn, \& Davis, 1996). Rosswurm and colleagues (1996) discussed Appalachians and illness, noting sickness or debility may be viewed as "normal" aging, or the "will of God", with heavy reliance on family to provide care (p.443).

In response to the challenges of rural life, the Appalachian Regional Commission (ARC), founded in 1965 , was created to address poverty in the region by diversifying the economy and providing better health care and educational opportunities to the people (Ziliak, 2010). In the Executive Summary of Health Disparities in Appalachia, the ARC reports that despite the Appalachian region making progress in lowering poverty rates and unemployment rates, while 
increasing secondary education levels and income amount, Appalachian areas continue to suffer with poverty and poor health compared to the entire United States [US] (2018). Mortality rates are incongruent between Appalachia and the rest of the US, with the overall Appalachian region having a 25\% higher rate (ARC, 2018). Compared to the US, Appalachia has higher rates of heart disease (17\%), Cancer (10\%), Chronic Obstructive Pulmonary Disease (27\%), Injury (33\%), Stroke (14\%), Diabetes (11\%), Suicide (17\%), and Drug overdose (37\%) (ARC, 2018, p.

5-8). Persons receiving Medicare living in Appalachia report a 16.7\% higher rate of depression than the rest of the US (ARC, 2018). The ARC reports social determinants of health are perversely lower in Appalachia than US and many state social determinants of health (median household income, household poverty rate, percentage of population receiving disability benefits, percentage of population with some level of college education, and social association rate). Health outcomes are worse in Appalachia. The ARC reported that socioeconomic insufficiencies and health deficiencies are interconnected, contributing to health disparities. With a cultural focus on community and kinship, use of available resources may be self-limiting in this culture and attempting to understand factors that contribute to non-use of resources could assist nurses and other clinicians in providing culturally-sensitive information and resources.

\section{Significance of Caregiver Non-Use of Formal and Informal Services}

Use of support services by caregivers has been shown to help PWD continue to live in the community. By better understanding explanations of service use or non-use, nurses can screen caregivers at risk for problems, intervene with education about available resources in an informed manner, and offer more individualized assistance for the caregiver and care recipient dyad living in Appalachia. To better understand how to address CG needs for support services, the factors that impact non-use of services must be identified in Appalachia. 


\section{Major Components of the Study}

Purpose: The purpose of this study was to explain factors most strongly associated with caregivers' non-use of formal and informal services in Appalachia.

\section{Research Questions.}

1) What estimate of the variance in the non-use of services in Appalachia can be explained by the predisposing characteristics of demographic variables, social variables, and belief variables?

2) What estimate of the variance in the non-use of services in Appalachia can be explained by enabling factors of financial and organizational resource variables?

3) What estimate of the variance in the non-use of services in Appalachia can be explained by the need factors, both perceived and evaluated needs variables?

4) What combination of predisposing, enabling, and need factors constitutes the strongest explanatory or associative model of non-use of services?

\section{Definition of terms.}

Caregiver (in the survey and analysis) is the unpaid (informal) family member providing care including responsibilities for the person with dementia which he or she can no longer accomplish without assistance. Characteristics of the study sample are included in Table 1 (Appendix B). The caregiver had to assist the person with dementia in some manner with household activities (e.g. housework, meal preparation, supervising outside services), personal care (e.g. hygiene, grooming, toileting), managing finances, shopping and transportation, emotional or physical support (e.g. social activities, cheerful friend), or monitoring care (e.g. evaluating treatment outcomes, administering medications). 
Care Recipient (CR) is the person with dementia as diagnosed by a health care provider who requires assistance with daily activities, or help processing or understanding information.

Person with Dementia (PWD) is the care recipient who has been diagnosed by a health care provider as having dementia.

Predisposing characteristics are demographics, social characteristics, and health (dementia) beliefs. Demographic characteristics included CG age and CG gender. Social characteristics include CG level of education, CG relationship to PWD, and Faith/Spiritual beliefs that assist CG in decision making. Social relationship support is measured by The Social Provisions Scale Short Version (Cutrona \& Russell, 1987; Russell, Cutrona, Rose, \& Yurko, 1984).

Health (Dementia) Belief, in this study identified as beliefs about dementia, according to Phillipson, Magee, and Jones (2013) and as defined by Andersen's model is a predisposing individual characteristic and includes values, attitudes, and knowledge people have about health (dementia) beliefs that influence their perception of dementia treatment, stigma of dementia, and knowledge about dementia progression, and use or non-use of services.

Service (Dementia) Belief according to Phillipson et al. (2013) [adapting Andersen's model] is a predisposing individual characteristic and includes the CGs values, attitudes, and knowledge about dementia services. The CG may believe a service is or is not useful, or potentially harmful to the PWD.

Enabling Financing and Organizational variables are financial and organizational capabilities such as income, health services available, and access to the services as part of 
the Andersen model individual characteristics. Enabling financial variable addresses a need for help understanding or managing finances. Enabling organizing addresses the total caregiving services available (both formal and informal) and three subscales of the UNA including family concerns, emotional support, and finding services.

Evaluated Need is a health care professional evaluation of a need, such as a need for a higher level of medical care or durable medical equipment. In this study, evaluated need includes a combined variable including having a health care provider, the health care provider recommending services to the CG for the PWD, and both formal (paid) and informal (volunteer or unpaid) hours help received per week.

Perceived Need is an individual characteristic indicating the factor a person believes he or she lacks and needs help to overcome or to achieve, such as a caregiver recognizing he or she needs assistance with the care recipient's activities of daily living. Perceived need is measured in this study by combining scores of three subscales from the Unmet Needs Assessment (UNA) (Bass et al., 2012) health information, daily living tasks, and living arrangements as well as the total score from the Caregiver Mastery Subscale Revised (Lawton, Moss, Hoffman, \& Perkinson, 2000) that assesses the caregivers perceptions of ability to provide care More information on measures can be found in Chapter 3 . Non-Use of Services (Dependent Variable(s) are support services not used by the CG. Formal (paid) and informal (unpaid) support services were analyzed as 14 services (formal and informal combined), eight formal services individually, six informal services individually, and a dichotomous variable of users (not using 1-8 services) and non-users (not using 9-14) services. 


\section{Method}

A quantitative method using validated self-report surveys fits the study purpose to explain factors most strongly associated with Appalachian caregiver non-use of formal and informal services in Appalachia. A sample of family caregiver/care recipient dyads living in Appalachia was recruited from physician's offices, health care clinics, dementia support groups, geriatric associations, the West Virginia Alzheimer's Association, and other community groups via flyers, online website notices, specific email addresses from the named services, community contacts, and pamphlets. Recruitment was focused in Appalachia, primarily West Virginia and the measures

Appalachian counties. Inclusion criteria specified that the care recipient was an adult over 18 years of age, diagnosed with dementia by a health care professional, and not suffering from cognitive impairment from brain cancer or acute causes, such as cerebrovascular accident or traumatic brain injury.

Social characteristics were measured via demographic questions as well as questions added from the Caregiver Research Network: Benjamin Rose survey. The "Unmet Needs Survey" questionnaire was added en bloc to the survey, (Bass, Clark, Looman, McCarthy, \& Eckert, 2003; Bass et al., 2012) measured caregivers' health beliefs about dementia, and health service beliefs.

Financial characteristics were measured by the "Unmet Needs Survey" questionnaire added en bloc to the survey (Bass et al., 2003; Bass et al., 2012). Organizational characteristics were measured by questions added from the Caregiver Research Network Benjamin Rose survey. The Philadelphia Geriatric Center Short Length Multi-Level Assessment Instrument (PGC-MAI), now identified as the Caregiver Appraisal Measure (CAM) Caregiver Mastery 
Scale, was used to measure perceived need (Lawton et al., 1982; Lawton et al., 2013). Questions from the "Unmet Needs Survey" questionnaire (Bass et al., 2003; Bass et al., 2012) were used to measure evaluated need.

The collected data were coded and entered into SPSS software. Data were analyzed using descriptive statistics and logistic regression. The desired sample size of 157 caregivers of persons with dementia was originally calculated based upon the percent of population of West Virginians who are caregivers of persons with dementia, estimating a ten percent $(10 \%)$ return rate for mailed surveys. As this study was not a randomized clinical trial, a sample calculation based on population was considered appropriate. Additional information on sample size calculation is included in Chapter 3. A large sample was not attainable, possibly due to the burden of caring for a PWD, and with committee approval, data collection was ended after 14 months at 43 dyads.

The research questions were then analyzed by multiple regressions of independent variables (demographic, social, health (dementia) beliefs, service beliefs, financing, organizing, perceived need, and evaluated need) on the outcome of Non-Use of Services. The final sample of 43 dyads was used for the calculations, and due to the strength of the effects, the study demonstrated power greater than 0.8 in analyses.

Theoretical Rationale. Andersen's Behavioral Model of Health Service Use (Andersen, 1995; Andersen, 2008; Phillipson et al., 2013) was the theoretical model used to guide the study. Andersen's Behavioral Model of Health Service Use indicates use of health services is guided by predisposing, enabling, and need factors (Andersen, 1995; 2008). The Behavioral Model of Service Use began in the late 1960 s as a method to address, define, and measure equal access to health care (Andersen, 1995). Andersen's model evolved to include both individual and contextual determinants of health service use. 
The current study focused on individual characteristics identified in the model and was not designed to test the contextual characteristics also identified by Andersen (2008). Contextual characteristics incorporate systems, such as hospital organizations and health policy, which were not included because the focus was on the caregiver/care recipient dyad in the community setting (Andersen, 2008). Theoretical substruction was applied to Andersen's model as a dynamic process, thus allowing beginning research to determine relationships among variables to clarify the results of theory testing (Bekhet \& Zausniewski, 2008, p.206; Wolf \& Heinzer, 1999, p.33). The substructed model was appropriate to guide this study and included components that measured individual determinants of health service use, health care system resource use, personal health practices, health status outcomes, and provided a broad overview of the multiple individual components affecting use of health services in Appalachia (Andersen, 2008).

The health behaviors section of Andersen's model, including personal health practices, process of medical care, use of personal health services, and outcomes of perceived health, evaluated health, and consumer satisfaction are in the substructed model (Andersen, 2008). For the current study, the outcome variable was non-use of formal and informal services. Figure 1 is a model of the theory, extrapolated from Phase 5 of Andersen's Behavioral Model of Health Service Use (Andersen, 2008, p. 651). The variables were measured using validated and reliable instruments, and survey via questionnaire was a fitting method. Please see Figures 1 and 2 in Appendix C.

\section{Summary}

In summary, this chapter has introduced the background of the problem, definitions, theoretical framework, and the plan of the study. Framed within Andersen's Behavioral Model of Health Service Use and guided by four research questions, the study determined factors most 
strongly associated with non-use of services by family caregivers of persons with dementia in Appalachia. 
Chapter 2 Literature Review

The literature on caregivers of persons with dementia is vast, although studies of rural caregivers are less common. Chapter two includes an overview of dementia and a synthesis of the relevant literature. The methodological research review includes qualitative and quantitative information about caregiver use or non-use of services. Extant research findings related to the phenomenon of caregiving complete the chapter.

\section{Overview and Literature Search Process}

\section{Dementia.}

Caregiving in dementia is a complicated process, with variations related to the type and extent of dementia in the care recipient. The term dementia broadly describes a complex syndrome of progressive cognitive decline resulting from damaged neurons and includes impairment in at least two of the following areas: memory, communication, attention/focus, judgment or reasoning, and visual perception (Alzheimer's Association, 2015d; World Health Organization (WHO) and Alzheimer's Disease International (ADI), 2015). Cognitive impairment in dementia is significant enough to interfere with daily activities, and not part of normal aging (Alzheimer's Association, 2015d; WHO and ADI, 2012). There are many forms or types of dementia, and many disorders eventuate in dementia and Alzheimer's is just one of the etiologies; and, one presentation is not a stage of another type of cognitive disorder. There are some pharmacologic and behavioral therapies for dementia, but there are no cures, preventives, or treatments that effectively halt the progression for a predictable period in all persons with dementia (Alzheimer's Association, 2015e).

The healthy brain coordinates and regulates actions, thoughts, and life sustaining 
processes incredibly rapidly, relying on billions of neurons to communicate seamlessly.

However, aging, trauma, neurotoxins, inflammation, genetic abnormalities, vitamin deficiencies, hormone inconsistencies, nutritional imbalances, and other insults can interrupt the central nervous system and brain processes with devastating consequences (Daultzai, 2013; Rajmohan \& Mohandas, 2007; Rea, 2015). While there are some treatable disorders which can cause dementia, such as vitamin deficiencies and thyroid disorders, all signs of dementia do not necessarily disappear when the cause is treated. And occurring more commonly than treatable issues, there are many dementias which arise from irreversible or unknown origins (Alzheimer's Association, 2015d). Dementia is not a normal part of aging, but some degenerative changes do occur in the body and brain with increased age. Neurotoxins may initiate a more rapid gray matter atrophy and decreased cerebral volume leading to cognitive decline according to Daultzai (2013). Neurotoxins include aging, stress, depression, hypoxia (e.g. sleep apnea), hypertension, diabetes mellitus and insulin resistance, obesity, alcohol abuse, smoking, polypharmacy, oxidative damage, neurofibrillary tangles (abnormal Tau protein), beta amyloid overproduction, and systematic inflammation (Alzheimer's Association, 2015e; Cunningham \& Hennessy, 2015; Daultzai, 2013). All these factors and even more have been associated with dementias identified by the Alzheimer's Association.

\section{Types of Dementia.}

The most common type of dementia (more than 50\%) is Alzheimer's disease, followed by vascular dementia (formerly known as_multi-infarct or post-stroke dementia accounting for at least $10 \%$ of dementias) (Alzheimer's Association, 2015d). Alzheimer's dementia (AD) is caused by abnormalities in Beta Amyloid protein deposits in plaques, and neurofibrillary tangles (abnormal Tau protein) (Alzheimer's Association, 2015d). Vascular dementia is caused by 
problems in the micro-vasculature, such as microscopic bleeding or blood vessel blockages in the brain. According to the National Institutes of Health (NIH), National Institute on Aging (NIA), and National Institute of Neurological Disorders and Stroke (NINDS), Lewy bodies are abnormal deposits of alpha-synuclein in the brain (2013). Other differences in Dementia with Lewy Bodies (DLB) include the presence of visual hallucinations, sleep disturbances in which the person with DLB acts out dreams (Rapid Eye Movement [REM] sleep disorder), and extreme sensitivity to certain medications atypical antipsychotics (NIH, NIA, \& NINDS, 2013).

Additionally, Dementia with Lewy Bodies (DLB) accounts for $10 \%$ to $25 \%$ of cases; other types of dementias such as Creutzfeldt-Jakob disease (CJD) are less common (Alzheimer's Association, 2015d).

Mild Cognitive Impairment (MCI) is a diagnostic category wherein clinical change is noticeable but does not interfere with daily life or independent function. Persons with MCI may progress to dementia, while others improve, or stay the same with symptoms which do not worsen. Those with moderate impairment are estimated to be two times more likely to develop full-blown dementia and severe cases are 4.5 times more likely to progress to dementia. People with Traumatic Brain Injury are at increased risk of developing Alzheimer's or other dementias (Alzheimer's Association, 2015d).

The type of dementia affects the course of the disease, and therefore is a factor in determining when the CG's presence is required for the safety of the patient, and also the length of time care giving needed; for example, CJD dementia is typically an acute illness, with a shorter life expectancy than other dementias, like AD and vascular dementia. 


\section{Stages of Dementia}

Many researchers and clinicians divide symptoms of dementia into progressive stages based upon gradual decline in memory, judgment, communication, mobility, eating (appetite, chewing, swallowing), in addition to incontinence and possible behavioral disturbances (Alzheimer's Association, 2015e; Gauthier et al., 2006; Hamuro et al., 2007; Masumi et al., 2003; Prichep et al., 2006). By understanding stages of dementia, the patient, caregiver, and health care provider may anticipate changing needs of the person with dementia and recognize the need for additional treatment and services.

Barry Reisberg, MD of New York University identified seven clinical stages of Alzheimer's dementia (Reisberg et al., 2014; Reisberg \& Franssen, 1999). Reisberg's seven stages range from no impairment to complete dependence on another for care. The Alzheimer's Association alse has adopted Reisberg's framework as a useful construct (2015e). Stage 1 is normal, described as free of "objective or subjective symptoms of cognitive or functional deterioration, as well as absence of behavioral or mood disturbances" (Reisberg \& Franssen, 1999, p. 11). Stage 2 is described as normal forgetfulness, such as reported difficulty recalling names, and forgetting where objects were placed (Reisberg \& Franssen, 1999). Stage 3 (Reisberg \& Franssen, 1999) is mild cognitive impairment, such as asking repetitive questions, coworkers noting work difficulties, and some social impairment with organizing events or decreased concentration. In some individuals with cognitive impairment, no future decline was noted beyond minimal cognitive impairment; in others, progression of symptoms occurs over the next two to seven years (Reisberg \& Franssen, 1999). In these preliminary stages (2 and 3), a person with dementia (PWD) has some changes in the ability to think or learn, and forgetfulness, but can-do activities of daily living and carry on a conversation (Alzheimer's Association, 2015e). A 
PWD in the early stage may need support from friends and loved ones emotionally, but typically does not need a caregiver for daily activities such as grooming, bathing, eating, etc. He or she may need to transfer financial responsibilities to trusted loved ones, and to begin planning for the future changes that occur during the progression of the disease.

Reisberg and Franssen (1999) identified stage 4 as mild dementia, when cognitive impairment is noticeable to others, and the PWD loses some abilities to manage money, shop for groceries, and manage certain other activities of daily living. Throughout stages 3 and 4, the PWD begins having difficulty expressing thoughts, recalling information, and performing routine tasks (Alzheimer's Association, 2015e). The symptoms continue to increase in severity as the disease persists. Friends and family may observe behavior changes, and the PWD becomes confused in once familiar surroundings (e.g. lost while driving to the post office or walking in neighborhood of ten years residence) (Alzheimer's Association, 2015e). During stage 5, identified as moderate dementia, the patient's deficits in cognitive and functional abilities are impaired to the degree he or she is no longer able to live alone safely in the community (Reisberg \& Franssen,1999). A caregiver for a PWD may not begin to participate in daily care until the middle or late stages of the illness, but may assist with health care, financial activities, living arrangements, and safety concerns during the early time of the illness. Stage 6 , moderately severe dementia, describes a person unable to dress and groom himself or herself without assistance, and may develop incontinence. In stage 6 , the patient's memory is impaired to the degree he or she does not recognize current popular figures (such as the United States president) and may be unable to recall names of close family, prior schools attended, or may confuse the identities of family members (e.g. spouse for parent) (Reisberg \& Franssen, 1999). Finally, stage 7 is severe dementia, evidenced by limited speech digressing from a few spoken words to 
complete aphasia, impaired mobility worsening from needing assistance with ambulation to the inability to sit upright without support (Reisberg \& Franssen, 1999). Throughout the late stages of dementia (stages 6 and 7), the PWD remains confused much or all the time, has difficulty eating and/or swallowing, and requires constant care for his or her safety (Alzheimer's Association, 2015e). The use of services would be expected to escalate as the state of dementia increases.

\section{Trajectory of dementia.}

Disease trajectory provides a description of probable prognosis (time frame) and an outline of what is expected to happen throughout the rest of the illness (Murray, Kendall, Boyd, \& Sheikh, 2005). A time frame for dementias is notoriously difficult to accurately project, although the expectations for patterns of decline are more predictable. PWD live an average of four years from the time of diagnosis with the disease, although some persons have lived up to twenty years (Alzheimer's Association, 2015c). In terms of functional decline, patients may be expected to have progressive loss of memory, ongoing change in behavior and mood, and may experience movement disorders. In many persons with dementia, wandering and falls occur, and death is inevitable.

Bynum (2014) stated that no discussion of disease trajectory was complete without including consideration of prevention, risk factors, and treatment. However, in dementia, research has not yet clearly identified risk factors or preventive measures, other than that modifying risk factors for cardiovascular disease and diabetes may also favorably alter the risk of dementia (Bynum, 2014).

The medical care needs of PWD also change on a continual trajectory. Causes of cognitive impairment and dementias are multifactorial, and assessment, treatment of underlying 
sources of illness, and routine health maintenance are essential for care of the PWD. Exercise helps increase cerebrovascular blood volume, as well as assisting with control of hypertension and diabetes, and may play a role in neurogenesis (Daultzai, 2013). Non-pharmacologic prevention and control of risk factors includes managing stress, depression, sleep apnea, environmental toxins, and smoking (Daultzai, 2013). The cholinesterase inhibitors delay worsening of symptoms of dementias in approximately 50\% of PWD for six to twelve months (Alzheimer's Association, 2015c; Bynum, 2014; Daultzai, 2013). Some health care providers add alpha tocopherol (vitamin E) to the pharmacologic regimen for PWD, but the evidence is limited, and the drug interacts with other antioxidants, cholesterol medications, and anticoagulants (Alzheimer's Association, 2015c). Currently, behavior and sleep symptoms are treated with available medications on an individual basis, but risks of death with atypical antipsychotics, and fall risks with benzodiazepines and anticholinergic drug side effects are concerns (Alzheimer's Association, 2015c). These multiple ongoing physical and mental events make care coordination and hands-on caregiving for PWD extremely challenging, and services to assist in managing care in these multiple areas may not be available or known to the caregivers. Including a measure of availability of services is therefore important.

Adding to the complexity of planning for caregiving services is the increasing volume of people affected, along with the evolving treatment options and cost. In 2011, the Lewin Group developed a model estimating the number of persons in the United States living with Alzheimer's Disease from 2015 through 2050, assessing costs and disease severity (Alzheimer's Association, 2015a), including accounting for Garre-Olmo, López-Pousa, Vilalta Franch, Blanco, and Vilarrasa's (2009) report on neuropsychiatric disturbances in dementia (personality changes, psychomotor disorders, and disturbances in sleeping and eating). According to the 
Lewin Group projections for 2050, person with Alzheimer's disease in the United States (Alzheimer's Association, 2015c), there will be over 3 million in the early stages; 3.8 million in the moderate stage; and over 6.5 million in the severe stage. The projected costs in 2050 to Medicare and Medicaid is $70 \%$ of one trillion dollars, or more than one in five Medicare dollars (Alzheimer's Association, 2015a).

Research is focusing on earlier detection techniques, such as biomarkers, and newer pharmacologic treatments (Alzheimer's Association, 2015c). Newer drugs target neurotrophic factors, to assist with new neuron formation or to assist with countering the effects of neurotoxins like Beta amyloid excess, abnormal Tau proteins, inflammation, excess corticosteroids, and oxidative stress (Alzheimer's Association, 2015c; Daultzai, 2013). Daultzai (2013) suggested pharmacogenetics are be another route to new drugs, for those dementias with genetic components (e.g. Huntington's disease dementia). But currently, dementia treatment focuses on preparing the PWD and caregiver(s) for progressive loss of abilities during the illness, along with support of the caregiver to prepare for the stresses and changes, grief, loss, and end of life. A review of the varied factors surrounding dementing illness and the caregiving required for demented victims revealed that identifying services that could be used (were available) and were used by the caregivers was critical.

\section{Impact of dementia on caregivers}

The diagnosis of dementia impacts the PWD, his or her loved ones, coworkers, social systems, health care systems, and the financial and legal systems (WHO and ADI, 2012). Depending on the suspected etiology, there may be different predominant symptoms, but every dementia patient has devastating symptoms, such as difficulty remembering names, events (AD), problems organizing or making decisions (vascular dementia), movement disorders or 
hallucinations (DLB), or personality changes (Frontotemporal dementia [FTD]) (Alzheimer's Association, 2015e). After the PWD is diagnosed, he or she likely learn there is no cure, and very little symptomatic treatment available for a progressive illness leading to increasing loss of memory, communication, control of movement, and ability to care for oneself (Alzheimer's Association, 2015a; Bynum, 2014). The employed PWD may also lose his or her job, either through dismissal or forced early retirement (Flynn \& Mulcahy, 2013). Along with the inability to procure other employment, the loss of income, health insurance, contributions to pensions and retirement funds, and contributions to social security may lead to financial losses (Flynn \& Mulcahy, 2013). Most employers are not prepared to handle PWD and have only the Family Medical Leave Act (FMLA) in place as a resource for caregivers (Cox \& Pardasani, 2013). Caregivers of PWD (80\% are family members) must begin to adjust to changes in a loved one's personality, a changing relationship, shifting social, emotional, and financial situations, and often discover their own employment suffers as well (Cox \& Pardasani, 2013; Reed, 2012; Robinson, Clare, \& Evans, 2005).

\section{Caregiving Challenges}

Caregivers of PWD compared to caregivers of persons with other chronic conditions reported the need to acquire less demanding jobs, take early retirement, refuse promotions, or quit work because of the requirements of caregiving (Ory, Hoffman, Yee, Tennstedt, \& Schulz, 1999). Cox and Pardasani (2013) reported more than $64 \%$ of CG of PWD required changes at work, arrived late, requested to leave early, and/or asked for a leave of absence because of caregiving demands. Using data from 1996, Ory et al. (1999) findings suggested caregivers of PWD were more likely to give up pleasurable activities; had increased family conflict; and reported a higher level of emotional, physical, and financial strain than caregivers of persons 
without dementias. Certain caregivers of PWD have reported more use of services such as support groups, adult day care, nursing services, meal services, home modifications, and/or assistive devices than caregivers of persons without dementias (Ory et al., 1999). Equivalent data for CG in Appalachia has not been identified. The trajectory of dementia would suggest an increasing need for services. However, for caregivers in Appalachia, available services are less known; what services are being used and why are not known.

\section{Progressive Loss and Cost}

Dementia is a condition of progressive loss, and the caregiver and PWD, as well as their family and social network may experience multiple bereavements over time. Loos and Bowd (1997) suggest caregivers experience loss of control, less time for personal interests, decreased social activities and well-being, and occupational changes. Other losses include the loss of the PWD as he or she used to be, such as a problem solver, decision maker, discussion partner, sexual partner, or being independent (Ingebrestsen \& Solem, 1998). Impact on the social, healthcare, financial, and legal systems becomes entangled and compounded from both individual and family levels. As indicated in chapter 1, there are higher costs of healthcare associated with dementia, in part due to at least a doubling of hospital admissions compared to similar persons without dementia (Bynum, 2014). According to Bynum (2014), the Area Agencies on Aging and the Administration for Community Living assist many PWD with housing, and locally funded community-based services can support the elderly (including those with dementia), such as meals on wheels, caregiver services, day care centers, and senior centers.

Support of the caregiver to meet needs of the PWD can allow the PWD to avoid nursing home or long-term care placement. Bynum (2014) reports an average nursing home stay for oneyear costs around $\$ 75,000$, and over $75 \%$ of residents in nursing homes have a diagnosis of 
dementia. The Medicare system is typically responsible for hospitalizations. Coverage for nursing home or long-term care under Medicare is of very limited duration, however, and payment typically is transferred to the Medicaid system in long term chronic illness, such as dementia (Bynum, 2014). Financial support for caregivers is difficult to determine, with the need for and use of assistive services unclear.

Clearly PWDs living in the community have a progressively worsening illness that increasingly costs more healthcare dollars in provider visits, medications, personal supplies or equipment for care, costs of in-home or day care services, health insurance, and the majority of persons with dementias have more than one chronic illness (Bynum, 2014). The increasing number of PWD requires a growing quantity of persons able to provide care, using not only informal, but also formal (paid) services. Bynum in 2014 reported that currently there was an inadequate labor force, with low wages, and little to attract new talent to the formal caregiving field. Informal or family CGs may have limited access to formal caregivers as the number of PWD increases. The Alzheimer's Association Legal Plan (2015b) identifies the potential legal impact including a need for living wills, durable power of attorney, power of attorney for healthcare, guardianship/conservatorship, advance directives, a will, and a living trust. Caregivers of PWD need direction and support to access formal and informal support services, medical, legal, and financial expert knowledge to continue optimal provision of care. The challenges in understanding how use (or non-use) of services by caregivers of PWD is evidenced by the gaps in the literature review.

\section{Caregivers and Use of Services for Persons with Dementia}

The review of caregiver literature was completed based on the steps of a systematic review as well as an integrative literature review (Polit \& Beck, 2008; Torraco, 2005). An 
integrative review of the literature helped gain an understanding of measures of formal and informal services that may be available to caregivers of persons with dementia. This search included an analysis of relevant multidisciplinary studies of caregivers in the past 10 years. The review of literature for rural or Appalachian caregivers included an analysis of relevant multidisciplinary studies in the past fifteen years, in three separate literature reviews. Databases from West Virginia University libraries were: Academic Search Complete, Academic Search Premier, Ageline, CINAHL with Full text, Health Source-Nursing/Academic Edition, MEDLINE, PUBMED, PsycARTICLES, PsycINFO, and Women's Studies.

Keywords used for the literature search were rural, rural spousal caregivers, dementia patient, rural caregivers, dementia, caregiver, Appalachia, and Appalachia*. Each term entered into the keyword function was combined using the AND function. The researcher searched the following databases: Academic Search Complete, CINAHL with Full text, Health Source: Nursing/Academic Edition, Education Research Complete, Health and Psychosocial Instruments, MEDLINE, Mental Measurements Yearbook with Tests in Print PsycARTICLES, PsycINFO, PubMed, Social Work Abstracts, and Women's Studies. The researcher also conducted a separate search using Google Scholar. The search keywords were "multidimensional measures", “multidimensional assessment", "multidimensional instruments" dementia patient, dementia caregivers, dementia, caregiver, help seeking, use of services, use of social support, use of resources, service use, and resource use. Each term individually entered into the keyword function was combined using the AND function. Inclusion criteria included research articles from peer-reviewed journals, English language, human subjects, adult (ages 18 and above), quantitative measures, outcome of use or non-use of services and caregivers of persons with dementia residing in the community. The search netted 94 articles (excluding repeated articles) 
about CG of PWD, but many did not have "non-use" of formal and/or informal services as variables of interest. Please see Table 2 for inclusion and exclusion criteria for the literature review in Appendix D.

Articles not meeting the inclusion criteria listed above were excluded from review. The author read the full text of each remaining research article, paying attention to written purpose statements, investigator identified research questions and/or hypotheses, methods, and results sections. By reading the full text, the researcher identified the main ideas and themes of each article. The researcher removed one final article (Valle, Yamada, \& Barrio, 2004), because the variable was help-seeking behavior, and not service use. The researcher reviewed the reference lists of articles meeting inclusion criteria for possible articles published within the past 10 years but did not identify any additional references.

A strong theoretical foundation for the study was sought to advance the science, guide the research, and inform clinical practice (Alligood \& Tomey, 2010). According to Alligood and Tomey (2010), a theory organizes empirical knowledge into a formal explanation of a phenomenon. Walker and Avant (2011) describe the foundation of a theory as concepts. Theory helps expand the knowledge of a discipline, in this case, nursing. Finally, Walker and Avant indicate theory organizes relevant concepts and statements about a phenomenon into a theory, which is beneficial for "description, explanation, prediction, prescription [direction], and control" (p. 61). The review of literature for measures of caregiver use of resources identified three theories used in studying caregiver's use or non-use of services. The theories were Pearlin's Coping Stress Model (Sun, Roff, Klemmack, \& Burgio, 2008), Andersen’s Behavioral Model of Service Use (Phillipson et al., 2014), and the Theory of Planned Behavior with Andersen's Behavioral Model of Service Use (Phillipson et al., 2013). Andersen's model was 
chosen to guide this study because this theory addressed the characteristics that could potentially impact service use or non-use in Appalachian healthcare systems.

\section{Andersen's Model of Health Service Use}

Andersen's model has been used in many studies, and several systematic reviews of studies are available (Andersen, 1995; Andersen \& Newman, 1973; Babitsch, Gohl, \& von Lengerke, 2012). While there are hundreds of studies using Andersen's model, Babitsch and colleagues (2012) found a lack of consistency in associations in many of the study variables, and a need for further study. The authors noted the predisposing factors of demographic variables (age, marital status, and gender), social variables (education, ethnicity) as being most commonly studied. The more common enabling factors researched were income/financial situation, health insurance, and having a usual source of care/family doctor (Babitsch et al., 2012). The most commonly studied need factors included evaluated health status, and self-reported/perceived health, in addition to "a very wide variety of diseases" (Babitsch et al., 2012, p. 1). Phillipson and colleagues have used the model in both qualitative and quantitative studies (Phillipson \& Jones, 2011; Phillipson et al., 2013; (Phillipson et al., 2014). The study in 2013 by Phillipson et al. is a review of the literature regarding factors associated with respite services by caregivers in Australia. The model has not been previously applied to Appalachian caregivers.

\section{Predisposing, enabling, and need characteristics related to outcomes.}

Table 3, included in Appendix E, provides a summary of research studies, variables, and measures. Several studies reviewed included findings related to more than one of the individual characteristics from Andersen's model. The author organized the studies using the individual predisposing, enabling, and need characteristics, and the related findings for each variable (predisposing, enabling, or need) are included with the article review. 
Qualitative Studies. A review of available qualitative literature guided the identification of factors that relate to caregivers use or non-use of services when caring for PWD. StockwellSmith, Kellett, and Moyle (2010) indicated caregivers identified the physical care of the person with dementia as central to the caring role and did not wish to surrender these tasks for respite care providers (p. 2061). The authors' qualitative descriptive study of 16 CGs explored reasons why respite services in Australia were underutilized, solutions to the under-use, methods to help CGs find formal community services, and benefits and/or limitations in supporting the caregiving role (Stockwell-Smith et al., 2010). The researchers identified three themes from focus group data: commitment, needing help, and support (Stockwell-Smith et al., 2010). Commitment was identified as role reciprocity, definition, and frustration (Stockwell-Smith et al., 2010). Role reciprocity considered how the PWD would do the same for the CG if the positions were reversed. Definition identified the functions of a caregiver, such as a mediator between services or encourager to accept services or altered living arrangements (StockwellSmith et al., 2010). Role frustration described the challenges experienced as a CG, such as caregiving tasks, challenging behaviors of the PWD, and the time commitment required to obtain services (Stockwell-Smith et al., 2010). Needing help referred to the concerns of CG about the quality and safety of formal and informal resources, frustration of having to explain instructions to informal supports (e.g. how to give medications), and fear and resistance of losing a portion of the caring role by allowing others to assist (e.g. house cleaning services) (Stockwell-Smith et al., 2010). Stockwell-Smith and colleagues described support as informal networking, having misinformation or a lack of knowledge about available services for the PWD. Caregivers in the study suggested improving knowledge about available services, making access to the resources 
more navigable, and seeking CG/CR input for necessary resources would help with using respite care services (Stockwell-Smith et al., 2010).

Phillipson and Jones (2011) conducted a qualitative study in New South Wales, Australia to understand why many CG of PWD postponed use of residential respite care. There are two articles published describing the same study, the one in 2011 focusing on the research study, the 2012 manuscript a continuing education focus. The researchers (Phillipson \& Jones, 2011) used individual interview and focus groups guided by the Theory of Reasoned Action (Fishbein \& Ajzen, 1975 as cited in Phillipson \& Jones, 2011) and the Theory of Planned Behavior (TPB) (Ajzen, 1991 as cited in Phillipson \& Jones, 2011)) with 36 CG (N=36) of PWD. Phillipson and Jones (2011) explored behavioral, normative, and control beliefs using open-ended questions, which were audio-recorded, then transcribed, followed by a content analysis. The researchers used inductive analysis to explore the beliefs noted above, and Phillipson coded the initial interviews. Jones reviewed the themes identified by Phillipson (2011). Face validity and credibility of the analysis were completed using member checks during community forums, as well as with research participants. Findings of the study suggested CG who were non-users of services (or delaying use of services until the CR experienced further deterioration) reported they did not perceive a current need for services (behavioral belief), although they may in the future (Phillipson \& Jones, 2011). A second reported CG normative belief was that the service may result in a negative outcome for the CR (Phillipson \& Jones, 2011). The identified negative outcomes were deterioration in cognitive and behavioral function of the $\mathrm{CR}$, discord between the CG and CR, and CG guilt regarding placement of PWD in care (Phillipson \& Jones, 2011). Normative CG beliefs related to service non-use of residential respite care indicated the CG believed family should provide care for loved one and not place a PWD in care of others, and 
residential care services were low quality (Phillipson \& Jones, 2011). CG who were non-users of services described control beliefs that residential care services were unable to meet the needs of the PWD, the CR did not want to be placed in the facility, the residential care services would not be available when needed, and that to losing control of caregiving responsibilities was disagreeable (Phillipson \& Jones, 2011, p.704).

Phillipson and Jones (2011) described caregivers using support as having positive beliefs about the day center services. The researchers conducted a qualitative descriptive study of $36 \mathrm{CG}$ using focus groups and interviews to guide interventions promoting use of day centers for PWD (Phillipson \& Jones, 2011). Caregiver beliefs associated with the use of day centers were reported to include: PWD profit from social and mental stimulation, and achieve positive outcomes from a change in environment; CG must take care of themselves as well as the PWD by meeting social and practical needs, and need respite from caregiving responsibilities periodically; and a CG needed to overcome guilt associated with thinking he or she was not providing safe care for the PWD by using day center services. CG beliefs associated with nonuse of day centers included: potential negative outcome for the PWD in unfamiliar environment; using a support service indicated the CG was not meeting his or her responsibilities to the PWD; had no benefit for the CR or the CG; the CR refused to attend; and the day center was unable to meet physical or emotional needs of the CR (Phillipson \& Jones, 2011). In summary, the qualitative literature about individual predisposing characteristics suggested non-use of services included lack of knowledge and access to formal community services and barriers to use. Additionally, CGs not using services resisted relinquishing care to others. The CG belief that the respite service was unable to provide appropriate or quality care was another contributing factor to service non-use, or if the CG feared there would be a negative outcome for the PWD. 
Quantitative Studies. A review of quantitative studies was consistent with qualitative literature, expanded knowledge of what is known, and identified gaps in understanding caregiver non- use of services for PWD. Predisposing characteristics include demographics, social, beliefs about health, and beliefs about services (Andersen, 1995). Phillipson et al., (2013) conducted a quantitative descriptive survey using mailed questionnaires, as well as those distributed in person by participating medical and Alzheimer's Association Telephone helpline in Australia to identify factors associated with CG non-use of day care or residential respite services. The researchers utilized the Theory of Planned Behavior (Fishbein \& Ajzen, 1975 as cited in Phillipson \& Jones, 2011), within an expanded Andersen Behavioral Model of Health Service Use (Andersen, 1995) to obtain self-completed surveys from 113 caregivers of PWD. The researchers reported four hypotheses about the use of day or residential respite services: 1) Negative behavioral beliefs would be associated with non-use; 2) Negative control beliefs would be associated with non-use; 3) Negative normative beliefs would be associated with non-use; and 4) Negative service beliefs would be more strongly associated with non-use than negative health (dementia) beliefs (Phillipson et al., 2013, p. 413). Phillipson and colleagues summarized items about beliefs of health and services from past studies, as well as dichotomous questions about predisposing, enabling, and need variables (2013, p. 413-414). Survey questionnaire included predisposing factor questions for both CG/CR dyad about age, gender, relationship of CG to CR, co-residency, language spoken, 11 health belief statements (embarrassing to take PWD in public, selfconscious in pubic with PWD, avoid in home visitors, dementia as a mental illness, embarrassment about CR memory issues), four questions regarding the efficacy of dementia treatment including medications, and two questions about the government's role (provide more services, and help for at-home caregivers) (Phillipson et al., 2013, p. 413). Nine questions were 
also included about service beliefs, including the CG belief about potential positive outcomes for the $\mathrm{CR}$, benefits to $\mathrm{CR}$ and $\mathrm{CG}, \mathrm{CR}$ or family refusal to use services, access to services, suitability (centers able to meet physical and emotional needs of CR), and CG belief he or she must perform all care duties for PWD (or is not fulfilling CG tasks). Enabling factors were assessed using questions about CG income, educational level, availability of informal support in the caregiving role, and geographic location (Phillipson et al., 2013). Phillipson et al. (2013) measured need variables using questions about CR activities of daily living (ADLs), wandering, behavior, and cognitive status, and employed the Zarit Burden Screener (Bédard et al., 2001) and CES-D depression screener (Radloff, 1977) to assess CG need, assessing burden and depressive symptoms respectively. Two of their four hypotheses were supported: that negative behavior beliefs were associated with day or residential respite service non-use, and negative service beliefs were more strongly associated with service non-use than negative health (dementia) beliefs (Phillipson et al., 2013). Although CGs reported needing day and residential care respite, over $40 \%$ were not using day services, and $60 \%$ were not using residential services (Phillipson et al., 2013). The strongest associated factor for day (OR 13.11); 95\% CI $(3.75,45.89)$ or residential (OR 6.13); 95\% CI $(2.02,18.70)$ respite service non-use was the predisposing belief the respite use would result in a negative outcome for the CR (Phillipson et al., 2013). These studies suggest that non-use of services has a critical impact on care recipients.

Phillipson et al. (2014) conducted a literature synthesis guided by Andersen's Behavioral Model of Service Use, to determine factors associated with CG's non-use of respite services. Phillipson and colleagues reviewed 14 articles, including six cross-sectional studies; one longitudinal; one retrospective cohort; and one quasi-experimental pre- \& posttest intervention measuring factors associated with use of adult day centers. Additionally, they examined cross- 
sectional studies including two that used interviews and surveys measuring in-home and day center use as separate dependent variables, a CG survey investigating factors associated with short-term use of residential care, and one assessing study regarding factors associated with the use of day center programs and in-home services. A retrospective cohort study designed to examine variables influencing the use of 10 CG services was included (Phillipson et al., 2014). There were 10 types of CG services described by Douglass and Fox (1999) cited in Phillipson et al. (2014). Content validity was confirmed by having two researchers independently code for the variable "non-use of service" with a common data extraction form, and any discrepancies were reviewed by a third researcher. Reliability (internal consistency) was described when interpreting the quality of the research designs in assessing consideration of bias, statistical analysis, study design, comparison groups, length of follow up, a measure of eighty percent data completion at follow up, and consideration of the randomization process (Phillipson et al., 2014, p.3).

The 10 types of services were: "physician services, senior center services, homedelivered meals, home health care, homemaker-chore services, adult day care, case management, transportation services, residential care, and nursing home care” (Phillipson et al., 2014, p. 103). Also, 10 types of respite available to CG of PWD in the review were coded into four respite services: senior citizen centers; adult day care; short-term use of residential care; and nursing home respite used in the past 12 months (Phillipson et al., 2014, p. 4). Phillipson et al. (2014) reported non-users of respite service tended to be spouses, especially females over the age of 70 years. Obviously, if a CG was unaware of available services, he or she was a non-user (Phillipson et al., 2014). Likewise, if a CG did not believe a service was useful, he or she was a non-user of the resource. CGs living in less densely populated areas, and those who were embarrassed to be seen in public with the PWD tended to be non-users of in-home services 
according to Phillipson and colleagues. CGs with a sense of duty did not utilize day center respite services (Phillipson et al.). CGs using services were inclined to value reliability in schedules. Service non-use associated with predisposing variables CG/CR age and gender, and spouses were more prone to service non-use, especially with respite care (Phillipson et al., 2014). Non-spousal caregivers used more respite care resources than spouses did. Findings for ethnicity regarding use of services were mixed (Phillipson et al., 2014). Phillipson and colleagues (2014) reported use of in-home respite services was associated with embarrassment to be in public with the person with dementia, although not associated with day center use.

In summary, the quantitative literature about individual predisposing characteristics supported non-use of formal and informal services is affected by CG belief of negative outcome for the CR, and CG negative beliefs about service were more strongly associated with non-use than negative beliefs about health (dementia). The primary gap found in the research literature was the lack of studies in non-urban settings and no studies of CG for PWD non-use of services in Appalachia.

\section{Individual Enabling Characteristics Related to Outcomes.}

Qualitative Studies. Enabling characteristics include financial and organizational characteristics (Andersen, 1995). In a qualitative descriptive study, researchers employed semistructured telephone interviews of Australian CG of PWD to understand barriers to use of day respite and methods to facilitate use of the services (Robinson et al., 2012). The researchers reported credibility, dependability, confirmability, and transferability were established using criteria identified by Lincoln and Guba (1985). The $27 \mathrm{CGs}$ interviewed $(\mathrm{N}=27)$ included those caring for CRs refusing day respite $(n=10)$, and those attending the day services $(n=17)$. Researchers constructed six key interview questions, with optional clarifying questions for 
interviewer use in eliciting information as needed (Robinson et al., 2012). The six key questions asked about the decision to access day respite care, the assessment and referral process, if the CG or CR visited the center after the referral, knowledge about the center's facilities and activities, reasons for stopping attendance if appropriate, and the $\mathrm{CG} / \mathrm{CR}$ needs if attending or not attending the day center (Robinson et al., 2012). The researchers coded the findings into five themes: getting the correct information to access the center (being confused by information or feeling overwhelmed by the volume of information); feeling unsafe as an attendance barrier (leaving the comfort and safety of home, potential embarrassment to be seen by former colleagues or friends); reacting to the $\mathrm{CR}$ refusal to attend (all $\mathrm{CG}$ reported initial CR refusal); helping to ease the transition to the center (promoting safety and security, persevering in attendance); and benefits of attendance for the CG and CR (Robinson et al., 2012, p. 204). The findings indicated caregivers were at times overwhelmed by the magnitude of information (unable to organize), confused about the process of receiving in-home care, and worried about the care recipient's safety in new surroundings (Robinson et al., 2012, p. 196). In summary, the qualitative literature about individual enabling characteristics suggested CG needed correct, concise, and clear information about informal and formal services to use the resources and must feel safe when using the services.

Quantitative Studies. A quantitative cross-sectional descriptive study used self-report data to explore CG of PWD (N=62) experiences using respite care, including the motivation for using the service, impact on both the CG and CR, degree of satisfaction with the respite help, and request suggestions for enhancement (O'Connell et al., 2012, p.112). O'Connell and colleagues (2012) reported CG of PWD indicated the main reasons for use of respite services were to attend to $\mathrm{CG}$ own health care needs, cope with the duties of CG role, and to have time off from the 
tasks of caregiving. O’Connell et al. (2012) utilized The Carers Respite Survey (CRS) and the Carers' Perceptions of Respite Services Scale (CPRSS). The CRS included questions about demographics of the CG and CR (one form), and the first and second most commonly used forms of respite (O'Connell et al., 2012) in the previous 12 months. The researchers assessed frequency, cost, adequacy, CG satisfaction, and perceptions of the respite care on the CG and CR using the CPRSS (O'Connell et al., 2012). The CPRSS included 11 items with a five-point Likert rating scale, ranging from 1-strongly agree to 5-strongly disagree. The researchers added five open ended questions about what CG most preferred and least enjoyed regarding the respite service, the effect of the respite service on the health of the $\mathrm{CG}$ and the $\mathrm{CR}$, and suggestions for improving services (O’Connell et al., 2012). Although the variable was not exactly use of services, the study informs researchers about the reason for service use and suggestions for improvement. The Carer's Perceptions of Respite Scale has a reported $\alpha=0.84$, but there are no data available regarding test-retest reliability. Day care respite was the most used service $(\mathrm{n}=18$, $29 \%)$, followed by residential $(n=14,23 \%)$, then in-home $(n=9,15 \%)$, cottage $(n=6,1 \%)$, and finally regular outings $(\mathrm{n}=2)$. Of important note is that $13 \mathrm{CG}$ reported no preference for respite services (20\%). Respite services were either day center (day care in a community center), inhome, residential (day and night care for more than a few days), regular outings (excursions of 1 day), or cottage care (overnight care in a house or cottage for short periods of a day or weekend). CGs in the O'Connell et al. (2012) study suggested respite care services need a higher quality of care and administration, accommodating schedules, permanent staff, increased communication, and more affordable pricing. O'Connell and colleagues reported the main reasons for CG nonuse of respite care were the CG's own health, and CG needs regarding the caregiver role. CG who were employed outside the caregiver role tended to use respite services (O'Connell et al., 
2012). O'Connell and colleagues' study supported inclusion of available respite services and degree of CG satisfaction with the services, in addition to CG information about level of education, age, status of employment, and CG health issues. Findings from O'Connell et al. (2012) address enabling factors, as well as need variables from Andersen's model. CG health status and perceived needs were primary reasons explaining use or non-use of formal and informal services (O’Connell et al., 2012). O'Connell and colleagues' work suggested that studies of CG use of formal and informal services should include questions about respite care and CG degree of satisfaction with the services.

Canadian researchers conducted a cross-sectional descriptive study using a telephone survey $(\mathrm{N}=1,152)$ to gain a description of an awareness of community resources, and then used a vignette methodology to gather information from participants regarding hypothetical situations about caring for a parent with dementia (Ploeg et al., 2009). Vignettes, fictional short narratives that were similar to everyday situations, were used by the researchers to gain an understanding of the decision-making process of respondents and identified relevant use of resources in a narrative format (Ploeg et al., 2009). Dementia-care service providers developed the vignettes, which were pretested and modified as necessary, lending face and content validity (Ploeg et al., 2009, p. 362). A professional firm hired to conduct the telephone survey also coded the responses, collaborating with the researchers during coding (Ploeg et al., 2009). Content validity was assessed per peer check, and inter-rater reliability among professional firm and researchers when sorting interview data initially to 150 themes, then into 20 "meaningful" themes.

This study (Ploeg et al., 2009) was informative in use of resources categorized into meaningful themes: 1) Community support services (includes 37 agencies such as adult day center, Meals on Wheels, the Alzheimer's Society, and transportation services); 2) Spouse 
(informal support); 3) Son/daughter (informal support); 4) Friends and neighbors (informal support); 5) Relatives (informal support); 6) Physician; 7) Emergency; 8) Clinics/hospitals; 9) Other health professionals; 10) Non-health professionals; 11) Pastor/clergy/faith community; 12) Social and recreation services; 13) Nothing; 14) Home health services; 15) Long-term care/residential care; 16) Self-help/personal strategy (informal support); 17) Government; 18) Information and referral sources; 19) Disease-specific agencies; and 20) Community Care Access Centre (home health services) (Ploeg et al., 2009, p. 364).

Ploeg and colleagues (2009) then incorporated the 20 meaningful themes into 12 vignettes, and one of a possible three vignettes was read to respondents to answer three research questions about which resources to use when caring for a parent with dementia, factors associated with identifying those resources, and determining if CGs added awareness of resources (Ploeg et al., 2009). Participants identified resources to use when faced with caring for parent with dementia (from vignette) and were asked to identify community health and support services, as well as home health services, and physician use. Investigators asked respondents what they would do in the scenario, followed by the prompt "Anything else?" up to four times to collect the sources of help. Next, participants were asked to name an organization or community program, which could be helpful, again questioned up to four times, until the respondent mentioned a community service (Ploeg et al.) The respondents were a combination of CGs $(\mathrm{n}=$ 474) and non-CGs $(n=678)$. CGs identified the first choice of help as physicians $(25 \%)$, followed by informal sources (20\%), then home health services (19\%), community support services (9\%), and finally long-term care (8\%) (Ploeg et al.). The only statistically significant demographic variable was increasing age $\left(\chi^{2}[4, \mathrm{n}=1,152]=11.2, \mathrm{p}=0.03\right)$ (Ploeg et al., 2009, $\mathrm{p}$. 365). Ploeg and colleagues' results showed that participants identified the physician as the first 
choice of support (37\%), followed by informal support (33\%), with home health services the third selection (31\%). As CG age increased, they were less likely to suggest informal support resources. CGs in this study who were females were $40 \%$ more likely to identify the physician as a source of support, and if the CG had a higher level of education, the odds increased by $250 \%$ he or she would identify the physician as a source of support (Ploeg et al., 2009). Participants in the study who were married were less likely to identify informal support (Ploeg et al., 2009). Being a CG was not a statistically significant associated variable with identifying community health or support service resources (Ploeg et al., 2009). In summary, the quantitative literature about individual need characteristics related to non-use of services suggested age, level of education, and both formal and informal support identification as important study variables in understanding CG use of services.

Robinson, Buckwalter, and Reed (2005) performed a secondary analysis of data from a prior multisite longitudinal 1-year study titled the National Caregiver Training Project (Hall \& Buckwalter, 1987 as cited in Robinson, Buckwalter et al., 2005) that used repeated measures to answer two questions: 1) What is the strength and direction of relationships among CG and CR demographic variables and use of community services; 2) Of the variables identified in the literature as being relevant to use of services (severity of disease, problem behavior, and social support) and the demographic variables, which is the best predictor of use of community services? The sample consisted of $241 \mathrm{CG} / \mathrm{CR}$ dyads living in the community (Robinson, Buckwalter et al.). The researchers employed the Social Provision Scale (SPS) (Cutrona \& Russell, 1987). The SPS computed total score (sum of all items) reliability coefficient is $\alpha=0.85$, with reported $\alpha=0.53-0.70$ for each of four-item subscales (Robinson, Buckwalter et al., p. 131). Robinson, Buckwalter et al., 2013, also used the SPS scale. Robinson, Buckwalter and colleague 
reported variables effecting use of community resources were the CR frequency of ADL problems $(\mathrm{p}=0.003), \mathrm{CR}$ frequency of memory or behavior problems $(\mathrm{p}=0.012), \mathrm{CG}$ spousal relationship to $C R(p=0.001)$, and CG social support $(p=0.002)$. Spousal CG were significantly less likely to use services $(r=-0.232)$, but the strongest relationship with use of services was CR frequency of problem behaviors and memory problems (ADLs, $r=0.35$; memory problems, $r=$ 0.34). This differs from O'Connell et al. (2012), whose findings suggested CG health and needs were the strongest contributors to CG use or non-use of services. Researchers identified informal and professional resources used as respite service or caregiving assistance (Robinson, Buckwalter et al., 2005). Robinson, Buckwalter et al. (2005) reported caregivers who were spouses were less likely to use respite services.

Robinson, Buckwalter et al., (2013) later completed another secondary data analysis from the National CG Training Project $(\mathrm{N}=241)$ to answer four research questions about CG use of help, support groups, community service, and CG characteristics. The four questions were related to use of services: 1 . How much help do CGs use? 2. What percentage of CGs attend support groups; 3. What are the demographic characteristics with different patterns of community service use; and 4. What are the differences between users and non-users of community services in terms of CR behavior and CG depression and social support? (Robinson, Buckwalter et al., 2013, p.502). According to Robinson, Buckwalter and colleague, community service use was help from adult day care centers, home health care, respite care, therapists, social workers, nurses, and/or formal providers either inside or outside the residence of the PWD. Informal service was aid or support from unpaid persons, such as family, neighbors, friends, or volunteers. Robinson, Buckwalter et al. (2013) stated CG considering respite services reported 
feeling puzzled by the process, apprehensive regarding the volume of information, concerned about the CR safety, and anxious about public knowledge of the CR having dementia.

In the Robinson et al., (2013) study, the 24-item Social Provision Scale (SPS) (Cutrona \& Russell, 1987) measured perceived social support of the CG. Robinson and colleagues (2013) reported the SPS (Cutrona \& Russell) had "good" ( $\alpha=0.85-0.92)$ reliability across varying populations, with individual subscales ranging from $\alpha=0.64$ to $\alpha=0.76$ (Dukes Holland \& Holahan, 2003 as cited in Cutrona \& Russell, 1987). The researchers reported validity of the SPS was supported in studies by Cutrona et al. (1986), Russell \& Cutrona (1991), and Dukes Holland and Holahan (2003) via added factor analyses. The SPS, developed by Cutrona \& Russell (1987), measures how much social support one receives from social networks. Cutrona and Russell (1987) based the 24-item questionnaire on Weiss' (1974) six social provisions, attachment, guidance, social integration (feeling of belonging), opportunity for nurturance (helping others), reliable alliance (can depend on social network to be present in time of need), and reassurance of worth (Cutrona \& Russell, 1987). In the literature review, the SPS was thus the most frequently used instrument to assess caregivers and was important to include.

Services used by family CG in the study were community services, support groups, informal help, professional help, and adult day care (Robinson et al., 2013). Robinson and colleagues reported CR problem behaviors and relationships with CG were strongly associated with the CG use of services ( $p<0.001$, and $p=0.001$ respectively). CG using both caregiving assistance and respite were significantly younger than CG using neither service $(p=0.010)$. The average informal service use of CG in the study was nine hours per week, and four hours of professional help (in home or adult day care) (Robinson et al., 2013). Service non-users lived with PWD (78\%) and were spouses (77\%), were older, reported more depression, and received 
less social support (Robinson et al., 2013). Interestingly, the non-service user CG provided fewer hours of care to a CR with milder cognitive, functional, and behavioral complications (Robinson et al., 2013). Robinson et al., (2013) reported the level of problem behaviors of the care recipient was strongly associated with use of services. Robinson et al. (2013) findings suggested non-users were older, more depressed, received less social support, but provided fewer hours of care per week to the care recipient that had less cognitive and functional deterioration and fewer behavioral problems. Data collected by Buckwalter and analyzed by Robinson, Buckwalter et al. $(2005,2013)$ supported use of the SPS as a reliable measure of social support to include in a study of CG use/non-use of formal and informal resources. Appendix F Table 4 includes the Expanded SPS.

Expanding the Social Provision Scale (SPS). The SPS was the most frequently used instrument to assess CG use of resources in the recent literature (Robinson, Buckwalter et al., $2005 ; 2013)$, however the scale was not adequate for assessing all variables of interest in Andersen's model, or identifying important information suggested from the review of literature (Brown, Chen, Mitchel, \& Province, 2007; Johnston et al., 2011; O’Connell et al., 2012; Phillipson et al., 2014; Phillipson et al., 2013; Phillipson \& Jones, 2012; Phillipson \& Jones, 2011; Ploeg et al., 2009; Robinson, Buckwalter et al., 2005; Robinson et al., 2012; Robinson et al., 2013; Stockwell-Smith et al., 2010; Sun et al., 2008). Table 4 in Appendix F connects the literature review to the expanded SPS questions. For the current study, an expansion of the SPS was tested in a pilot study with former caregivers of PWD to have a more complete understanding of CG non-use of services. Volunteer former CG of PWD completed the SPS 24item questionnaire first (Cutrona \& Russell, 1987), included in Appendix G with permission from Dr. Cutrona (and other letters of permission for instrument use). The respondents then 
completed the Expanded SPS, included in Appendix H. Five West Virginian former caregivers of a loved one with dementia were asked to pilot the expanded SPS questionnaire, and three were returned. There were no suggestions of positive or negative aspects, and no suggestions for changes. While the information may be valuable, the collection of respondent comments may add to respondent burden. The 24 item SPS was included in the compiled questionnaire without a separate request for comments.

For this study, all selected instruments and questions were compiled into one questionnaire (Appendix I). Seven Appalachian former or current caregivers of loved ones with dementia were asked to pilot the full questionnaire and provide information regarding length of time for completion, positive or negative aspects, and suggestions for change. Five (5) questionnaires were returned to the researcher. The average time for completion was twenty-five minutes (ranged from 18 minutes to 30 minutes). In reporting potential problem areas, one respondent indicated the portion of the questionnaire in table format asking about resources was confusing. The comment was "I did not know if I should identify a service or not". There were no other suggestions for change or clarification. The table directions for the survey indicated that all services should be identified. This compiled questionnaire was used in the current study. In summary, the quantitative literature about individual enabling characteristics suggested CG age, level of education, residing with $\mathrm{CR}$ or not, and relationship to $\mathrm{CR}$ are important factors to include in the study of CG of PWD. The enabling factors supporting CG use of services are available physician services, CG awareness of available formal and informal resources, CR health insurance to pay for formal services, and an informal support with caring tasks. Individual enabling factors associated with CG non-use of services include the CG being older, a spouse, and lacking knowledge of available resources. 


\section{Individual Need Characteristics Related to Outcomes}

Qualitative Studies. Andersen described a need characteristic as being perceived and evaluated (1995). In a grounded theory study, Brown et al. (2007) noted help-seeking was preceded by the care recipient's change in behavior, health or relating patterns of the care recipient, caregiver, or family members for older husbands caring for wives with dementia. The change resulted in a perceived need for help with the caregiving role (Brown et al., 2007). Brown et al. (2007) proceeded to do a secondary data analysis in 2008 and added a grounded theory study discussed in the next paragraph.

Researchers Brown and Chen (2008) used data from two prior grounded theory studies to conduct a secondary content analysis of spousal caregivers to explore the help-seeking process of spousal caregivers. The sample consisted of $20(\mathrm{~N}=20)$ spousal $\mathrm{CG}$ of a PWD, who were aged 60 years or greater. Help-seeking was identified as a process that began in response to a change in behavior or health of the CG or spouse (PWD), and the CG perceived a need for help with the CR (Brown \& Chen, 2008). Brown \& Chen (2008) found older spousal male caregivers were at ease with others assuming caring duties. Participants in the study underutilized both formal and informal services, but husbands and wives sought care differently. Husbands in the study tended to direct care provision by finding others to provide direct care or specific services, while wives performed most caregiving duties themselves (Brown \& Chen, 2008). Wives in the study were slower to recognize the symptoms of dementia in their spouses, thus delaying diagnosis. Both husband and wife $\mathrm{CG}$ preferred to pay for services rather than be beholden to friends or family for caregiving assistance (Brown \& Chen, 2008). Spousal CG of both genders reported feeling others were unable to provide the same quality of care to the CR. In summary, the qualitative literature about individual need supported gender as an important variable of study regarding CG 
use of formal and informal resources. Another reason CGs began sought formal and informal services occurred when the CR experienced a decline in health (both physical and mental). Thus, the CG gender and beliefs about the health of the CR are included in the current study.

\section{Quantitative Studies.}

Johnston et al. (2011) conducted a dual-purpose study. The first purpose was to assess a telephone screening measure in identifying persons with memory problems to assess those individuals with positive screens for possible memory problems in their homes. Purpose two was to develop a multidimensional needs assessment tool to determine the kind and number of unmet needs regarding persons with memory disorders. The researchers did not assess use of resources or services directly, however, the study informed the selection of a measure of service use because the findings helped identify unmet needs for the PWD and the CG, which could lead to CG use of resources. The categories of unmet needs suggest that resources for these needs should be available to the CG. There are no formal psychometric properties available for the Johns Hopkins Dementia Care Needs Assessment (JHDCNA); content validity can be found in the interdisciplinary party of dementia care experts guided by the best evidence-based practices in dementia care who developed the survey (Black et al., 2013). The JHDCNA assessed needs of CGs and CRs and established how well the needs were met. Black and colleagues encourage use of the instrument for clinicians and researchers (Black et al., 2012). Additionally, in another study the researchers demonstrated quality of life measures that had concurrent validity with the instrument developed by the researchers, the JHDCNA (Black et al., 2013). The top three unmet needs for a person with dementia $(n=13)$ were: 1$)$ a physical and psychosocial evaluation for dementia $(n=9 / 11) ; 2)$ general health care $(n=7 / 9)$; and 3) environmental safety $(100 \%)$ (Johnston et al., 2011). The top three unmet CG needs were: 1$)$ education about dementia $(n=5 / 6), 2)$ 
information about resources and services for PWD (n=3/3); and 3) CG mental health $(n=5 / 5)$

(Johnston et al., 2011, p. 296). In summary, the quantitative study findings suggested that a study of CG/CR should include assessing unmet needs for both members of the dyad.

\section{Outcomes Use/ Non-Use of Formal and Informal Services}

Qualitative Studies. There were no additional qualitative studies identified regarding the use of formal and informal services. Study findings identified earlier in the literature review identified individual predisposing and enabling factors could impact the outcome variable of use or non-use of formal and informal services.

\section{Quantitative Studies.}

Sun et al., (2008) analyzed existing data from African American and Caucasian CG (N= 720, $n=165$ male, and $n=555$ female), from the Resources for Enhancing Alzheimer's CG Health I (REACH I) project sites in Birmingham, Memphis, Philadelphia, and Boston. The dependent variables were use of formal services and use of informal support (Sun et al.). Formal service use included in-home services of homemakers, home health care, visiting nurses, meals delivered to the home, and out of-home services of transportation, day care, and group support. Sun et al. (2008) stated findings from the study indicated male caregivers used more in-home services than female caregivers, although females used more transportation services. Females in this study reported using more informal support resources.

Gitlin et al. (2003) developed a measure of use of formal services for the Resources for Enhancing Alzheimer's Caregiver Health (REACH) I study composed of seven questions about the CG using or not using a particular formal service within the last month. The REACH I and II studies were multisite, longitudinal research projects funded by the National Institute on Aging (NIA), REACH I September 15, 1995 through August 31, 2000, and REACH II September 30, 
2001 through September 31, 2004 (Stanford Medicine Older Adult and Family Center [SMOAFC], 2015). The researchers evaluated multi-component interventions on White, Hispanic, and African American caregivers of PWD (American Psychological Association, 2015). They reported a Cronbach's $\alpha$ of 0.60 for these questions. Informal support included "tangible support (help with transportation), emotional support (others listening or showing concern), informational support (offering suggestions)" (Sun et al., 2008, p. 4). An 11-item instrument based upon Krause and Markides (1990) publication (as cited in Sun et al., 2008) was used to measure informal support. Sun and colleagues reported a factor analysis of the items indicated "reasonable" loadings on a single factor, and a coefficient $\alpha$ of 0.81 (Sun et al., 2008, p. 4).

Brodaty et al. (2005) used findings from a literature review to identify CG motive for non-use of services and develop a typography of CG service non-use. The researchers then applied the typography to data collected from1991 to 1994 from the Victorian Carers Program (Brodaty et al., 2005). The Victorian Carers Program (VCP) was a national population based longitudinal survey conducted in Australia, investigating the effects of caregiving for persons with various “disabilities or special needs" (Schofield, Herrman, Bloch, Howe, \& Singh, 1997, p.60) to understand caregiving in the community. The VCP researchers used the Australian Institute of Family Studies' Computer Assisted Telephone Interviewing (CATI) system (Schofield, 1998; Schofield, Bloch, Nankervis, Murphy, Singh, \& Herrman, 1999; Schofield \& Herrman, 1993). The questionnaire for the VCP (Brodaty et al., 2005) incorporated questions from many instruments, including a measure of life satisfaction derived from Heady and Wearing (1992) (as cited in Brodaty et al., 2005), CG overload (Pearlin, Mullan, Semple, \& Skaff, 1990) and caring role resentment (Murphy et al., 1997). The authors were contacted to 
obtain more information regarding the measures used to construct the VCP survey, and other articles were obtained (Howe, Schofield, \& Herrman, 1997; Schofield et al., 1999 [refers reader to Schofield, Murphy, Bloch, Herrman, \& Singh, 1997 which was no longer in print for psychometrics and measures]; Schofield \& Herrman, 1993; Schofield, Murphy et al., 1997). Brodaty et al., (2005) reported that despite describing many unmet needs, one primary reason caregivers described for not seeking services was their belief they did not need the services. Two out of three CGs of PWD living in the community do not use supportive services or resources, and three out of four CG underutilize available support, despite reported unmet caregiving needs (Brodaty et al., 2005).

Schofield and Herrman (1993, p. 3) reported that their questionnaire was developed with the inclusion of measures of service use, however there were no psychometric properties reported for instruments in the 1993 article. In 1997, Howe et al. reported additionally that the respondent who self-identified as the primary CG identified those who helped with the caregiving, and further questions determined more information. The diagram displaying the questions are less about the resources used and more about the definition of the person providing most of care for the care recipient (Howe et al., 1997); nevertheless, the inclusion of resources can assist in identifying those services that need to be measured. Schofield, Murphy et al. (1997) included use or non-use of specific researcher-identified services. In summary, the quantitative literature about the outcome variable non-use of formal and informal service characteristics suggested the inclusion of researcher identified services as well as those identified in previous studies, and whether the service was used within a specific time frame.

Another quantitative study by Bass et al. (2003) and Bass et al. (2012) used their Unmet Needs Scale, based upon five constructs from the Stress Process Model as follows: 1) Primary 
objective stressors, 2) Caregiver context, 3) Primary subjective stressors, 4) Role and intrapsychic secondary strains, and 5) general well-being (Bass et al., 2012). The questionnaire measures help seeking by asking the CG or CR questions about the five constructs. The Unmet Needs Scale consists of 40 questions, and consists of eight subscales: 1) understanding dementia and its symptoms [7 questions], 2) care tasks [4 questions], 3) accessing services [6 questions], 4) legal and financial issues [4 questions], 5) organizing family care [6 questions], 6) alternative living arrangements [3 questions], 7) emotional support [4 questions], and 8) medications and medical follow-up [6 questions] (Bass et al., 2003; Bass et al., 2012). The Unmet Needs Scale has good structural validity, with factor loadings from 0.63 to 0.84 , and reported reliability of Cronbach's $\alpha=0.92$. Individual items measuring strain were scored from 0 to 3 ("strongly agree" to "strongly disagree") and independence and structural validity was confirmed by factor loadings ranging 0.42 to 0.75 on their respective factors (Bass et al., 2003; Bass et al., 2012). Emotional strain has a Cronbach's $\alpha$ of 0.87 . Health strain Cronbach's $\alpha$ is 0.83 . Relationship strain has six items and a Cronbach's $\alpha$ equal to 0.78 . Social isolation has a Cronbach's $\alpha$ of 0.90. The Unmet Needs Scale is relevant to perceived needs within Andersen's model. The subscale Understanding memory problems relates to predisposing health (dementia) beliefs; Accessing services relates to enabling organizing characteristics as does Organizing family care; and Legal and financial questions relate to enabling financing variables. All eight subscales relate to the outcome of service use, therefore the instrument fits within the theoretical model of the current study and was relevant to the variables of interest.

The current study added recommendations from the Family Caregiver Alliance National Center on Caregiving (FCANCC) about questions for assessments of caregivers (2012), including measures of CG resources (FCANCC, 2012). As no single measure was more highly 
used or recommended than another to measure CG use of informal and formal resources, the FCANCC and Benjamin Rose "Selected caregiver assessment measures: a resource inventory for practitioners" (2012) guided selection of resources for the current study. Appendix I contains the current study questionnaire with all included instruments.

\section{Summary and Theoretical Rationale}

The study sought to understand the contribution of predisposing, enabling, and need factors to formal and informal service non-use by Appalachian CG of persons with dementia. Chapter two included a description of the literature search, with the process for inclusion and exclusion of studies. Recent theoretical models used to guide caregiver non-use of formal and informal services research, as well as Appalachian or rural caregiving were identified, and Andersen's model selected for this study. The theoretical model including the variables is provided in Figure 2, Appendix C. Figure 3 in Appendix C includes the Measures from Andersen's Behavioral Substructed Model of Service Use. Qualitative and quantitative literature of caregiver non-use of formal and informal services revealed many quantitative studies had been conducted, but none were in Appalachia. The literature synthesis of studies within the past 10 years supported the selection of variables within the framework of Andersen's Behavioral Model that had a potential impact on the outcome of non-use of services. The next chapter will discuss methodology. 


\section{Chapter 3 Methodology}

Chapter 3 provides the descriptions of the research design, population, sample selection, instrumentation, and data analyses of Appalachian family caregivers of persons with dementia non-use of services. This chapter also describes the feasibility, advantages, and limitations of the proposed design, as well as ethical concerns and rigor of the study.

\section{Research Design}

The study used a correlational explanatory design (Frankel \& Wallen 2000). Data collection occurred from August 2016 to October 2017 with Appalachian caregivers responding at one point in time to a questionnaire integrating psychometrically tested instruments. The study did not incorporate an intervention. Correlational explanatory design using self-report survey was appropriate to answer the research questions because survey research is useful in collecting data about a population from individual persons (Polit \& Beck, 2014). Correlational data reported for multiple variables is found in Appendix J. All participants were analyzed as a single group. Data for the individual variables were collected from each participant. Outcomes using correlational explanatory design did not establish causal relationships, but rather suggested the strongest combination of predictive, enabling, and need factors explaining non-use of services by CG of PWD (Fraenkel \& Wallen, 2000).

An apriori sample size calculated using G-power considering a medium effect size, with eight independent variables from the Andersen model, suggested a sample size of 175 caregivers for a power of 0.8 would be needed (Faul, Erdfelder, Buchner, \& Lang, 2009). The eight variables included independent variables of predisposing (demographic, social, health beliefs , and beliefs services), enabling (financing and organizing), and need (perceived and evaluated) factors, and the dependent variable outcome non-use of formal or informal services. An 
alternative calculation of 157 caregivers needed was based on the 2010 population of $1,852,994$ people in WV (West Virginia Bureau for Public Health Statistics Center, 2015); and the estimated 36,000 West Virginians living with dementia. The Alzheimer's Association in the Latest Facts and Figures (2015a, p. 251) reported an estimated 58\% of older adults with dementia lived in the community with caregivers. Therefore, $58 \%$ of 36,000 West Virginians would be 20,880 PWD in WV including 75\% living with a caregiver, approximates 15,660 caregivers of PWD live in WV. Considering an expected $10 \%$ response rate to a mailed questionnaire (Funkhouser et al., 2017; Sadler, Lee, Lim, \& Fullerton, 2010; Sinclair, O’Toole, Malawaraarachchi, \& Leder, 2012), a sample size of 157 was the potential number of completed surveys based on WV population data. However, as has been found in previous research with this population and with health care surveys, the response rate was low, and it was not possible to attain the desired sample size (Sinclair et al., 2012). After 14 months of data collection, completed surveys from 43 dyads in Appalachia had been obtained, data collection was ended, and data analysis began. The sample was satisfactory for this study based on Beta weights in the analyses. Beta is the probability of Type II error in the hypotheses tests (incorrectly concluding no statistical significance), and since 1 - Beta can be considered as the power, the strength of the analyses for the sample of $43 \mathrm{CG} / \mathrm{CR}$ dyads would be greater than 0.80 (range of betas from .04 to .188) (Faul, Erdfelder, Lang, \& Buchner, 2007).

\section{Population and Sample Selection.}

The purposive convenience sample of 43 informal (unpaid) family caregivers was recruited from West Virginia and surrounding Appalachian counties via caregiver support groups, geriatric clinics, primary care clinics, advertisements in caregiver newsletters, ads on caregiver websites, Appalachian county area agencies on aging, home health agencies, and 
senior centers within Appalachian counties. An email was sent to the identified recruitment sites identifying inclusion and exclusion criteria to providers, support group leaders, clinic office personnel, agencies, and senior centers. Advertisements identified inclusion criteria as well as phone numbers to contact researchers for questions. Prior to securing informed consent, the researcher reviewed inclusion and exclusion criteria to self-report eligibility. Appendix K contains a table of inclusion and exclusion criteria.

A map of West Virginia (and the surrounding Appalachian counties) with counties of residence identified by the respondents is provided (Appendix L). As indicated in Table 3 (Appendix E), 43 CG of PWD had a mean age of 61.78 years $(\mathrm{SD}=11.66$; range 31-90 years); the mean age of the PWD was 80.13 years $(\mathrm{SD}=9.42$; range $61-95$ years). In the sample, 42 of the 43 CG/PWD dyads identified as Caucasian (Non-Hispanic) with only one CG/PWD dyad identifying themselves as African American (Non-Hispanic) race. There was no variance in language spoken in home - all spoke English. The most common relationships of CG to PWD were Adult Child ( $n=19,44.2 \%)$, and Spouse or Partner $(n=13,30.2 \%)$.

Inclusion Criteria. The inclusion criteria were that the caregiver had a family identifiable relationship to the care recipient and resided within an Appalachian designated county per the Appalachian Regional Commission designation. Further inclusion requirements were that the caregiver was age 18 years or older and spoke/read/comprehended written and spoken English. The care recipients were adults over the age of 18 years and had been diagnosed with dementia (either Alzheimer's or related dementia [ADRD]) by a health care provider.

Exclusion Criteria. The exclusion criteria included a neurocognitive impairment known not to be dementia (such as mass, traumatic brain injury without dementia, etc.); either the 
caregiver or care recipient lived outside the state of West Virginia (WV); or the care recipient had not been diagnosed by a health care provider as having dementia. The instructions stated only one caregiver for a person with dementia was to complete the survey.

\section{Instrumentation}

Demographic data were collected using a researcher designed demographic assessment guided by Andersen's model with information from the Family Caregiver Alliance: National Center on Caregiving and the Benjamin Rose Institute on Aging (2012). The literature review identified measures used in the past 10 years, and surveys/subscales of questionnaires to elicit relevant information, based upon psychometrically sound evidence, guided by the Andersen model, and keeping in mind respondent burden. The psychometrically validated measures were combined into one survey and completed by the CG to evaluate predisposing, enabling, need, and outcome measures for the proposed study. Table 2 in Appendix D reflects measures selected following the literature review.

\section{Predisposing Measures}

\section{Demographics and Social Variables.}

All studies reviewed collected demographic data; items chosen for the survey for the proposed study were guided by Andersen's model, consistent with Phillipson et al. $(2013,2014)$ use of Andersen's model. Demographic characteristics included CG age and CG gender. Social characteristics included CG level of education, CG relationship to PWD, and Faith/Spiritual beliefs that assist $\mathrm{CG}$ in decision making. Social relationship support is measured by The Social Provisions Scale Short Version (Cutrona \& Russell, 1987; Russell \& Cutrona, 1991). The Social Provisions Scale Short Version includes 10 questions to which the CG responds on a four-point 
Likert scale from 1 Strongly Disagree to 4 Strongly Agree regarding having people to depend on if he/she is in need, not having close relationships to others, not having anyone to turn to for guidance during stress, believing there are people who enjoy similar social activities, thinking others may not respect the CG skills and abilities, if something went wrong, no one would come to the $\mathrm{CG}$ assistance, having close relationships that provide CG with a sense of emotional security and well-being, having relationships where competence and skills are recognized, no one sharing CG interests and concerns, and CG believing there is a trustworthy person to whom the CG could turn for advice if there were problems (Cutrona \& Russell, 1987).

\section{Beliefs about Health (Dementia) Variable.}

Health (dementia) beliefs identified by Phillipson et al. (2013) included 12 questions about the CG beliefs about dementia were measured via a four-point Likert scale, ranging from 1- Strongly Disagree to 4- Strongly Agree, with a reported $\alpha=0.64$. Validity measures were not reported for the questions from Phillipson et al. (2013).

\section{Beliefs about Service (Dementia) Variable.}

Beliefs about service were measured using 12 additional questions reported in Phillipson et al. (2013), incorporating two questions about government service (dementia) beliefs, and 10 questions about dementia service beliefs. The questions were about CGs values, attitudes, and knowledge about dementia services. The CG may believe a service is or is not useful, or potentially harmful to the PWD (Phillipson et al., 2013). The questions were measured on a 4point Likert scale, ranging from 1- Strongly Disagree to 4- Strongly Agree. The questions about government were also measured on a 4-point Likert scale, 1- Agree or Strongly Agree, 2-

Disagree or Strongly Disagree, $\alpha=0.66$. There was no additional reported information regarding validity for the questions. 


\section{Enabling Measures}

\section{Enabling Financing.}

Enabling financing was assessed using combined variables from the Phillipson et al.

(2013) CG level of income question, plus the UNA (Bass et al., 2003; 2012; 2013) total subscale scores for UNA Legal Financial (UNALF). All questions in the UNA instrument ask if the CG needs more information or assistance with a topic and was answered with a 1- yes or 2- no. Dr. Bass granted permission to use the entire instrument for the study. See Appendix G for permission emails. The Legal and Financial questions addressed if the CG needed more information or help with managing the PWD bank accounts, updating wills, advanced directives, and power of attorney, or assistance understanding Medicare or Medicaid, or help with paying services not covered by insurance (Bass et al., 2012). This information about the CG need for help or information about legal and financial issues, used a yes or no answer scale (Bass et al., 2003; 2012; 2013). The Phillipson et al. (2013) CG level of income question was specifically about Australian income (less than $\mathrm{AU} \$ 30,000$ or equal to or more than $\mathrm{AU} \$ 30,000$ ) and was modified to reflect United States (US) dollars $(1=$ under $\$ 10,000 ; 2=\$ 10,000-\$ 39,999 ; 3=$ $\$ 40,000-\$ 79,999 ; 4=\$ 80,000-\$ 124,000 ; 5=\$ 125,000-\$ 150,000 ; 6=$ Over $\$ 150,000 ;$ there was an option "would rather not say"). Appendix M includes the UNA in entirety. The Phillipson et al. (2013) level of income was added to the score from the UNALF subscale total score. There were no published reliability and validity data for the level of income question to measure enabling financing. The questions from the Unmet Needs subscale Legal and Financial questions were measured for the instrument, not individual subscales. 


\section{Enabling Organizing.}

Enabling organizing is measured by combining the total caregiving services available (both formal and informal) and three total scores of subscales of the UNA including Family Concerns (UNAFC), Emotional Support (UNAES), and Finding Services (UNAFS) (Bass et al., 2012). The Caregiving Services Available include 14 services the CG identified as "yes, available in your area; no, not available in your area; and don't know if they are available in your area" and include eight formal services and six informal services. Family concerns (FC) included needing information or help about caring for the PWD, discussing memory problems and/or the course of dementia with others, help with caring for the PWD, dealing with family disagreement about care for PWD, and accepting the diagnosis of dementia. Emotional support (ES) evaluated the CG need for information or help finding someone to speak with who understands the CG situation, getting ES or counseling for the PWD and/or the CG. Finding services (FS) assessed if the CG needed information or help finding services or asking the health care provider for assistance, finding county services such as Area on Agency on Aging (AAA) or a local Alzheimer's chapter, getting the PWD to accept help from other service providers, getting health care providers to work together, finding services, and getting transportation to support services.

\section{Need Measures}

\section{Perceived Need Variable.}

Perceived Need was measured by a calculated variable combining total scores from three subscales of the UNA (Bass et al., 2012) and the Caregiver Mastery Subscale Revised (Lawton et al., 1989; 2000; 2013). The three subscales of the UNA are Health Information (UNAHI), Daily Living Tasks (UNADLT), and UNA Living Arrangements (UNALA). The UNA Health information (HI) included the CG needing information or help trying things to prevent worsening 
of dementia, keeping the PWD healthy, diagnostic tests for dementia, memory problems, the causes of dementia, dealing with other health conditions the PWD has, making plans for the future of PWD, taking the right medications at the right times, understanding how medications are supposed to help, possible medication side effects, future course of PWD and memory problems, keeping written notes to guide talks with health care providers, getting care instruction for PWD, and scheduling follow up visits with health care providers (Bass et al., 2012). The UNA Daily living tasks (DLT) included questions about the CG needing more information or help with daily chores, bathing or dressing the PWD, having a safe place for PWD to live, finding someone to stay with PWD so he/she are not alone, and stopping the PWD from driving a car (Bass et al., 2012). The UNA Living arrangements (LA) contained questions about the CG needing more information or help with where the PWD can live if he/she cannot stay in current living situation, paying for assisted living or long-term care, and getting information about assisted living or long-term care (Bass et al., 2012). The Caregiver Mastery subscale assesses the caregivers guilt about needing to do more for PWD, uncertainty about what to do about PWD, how often the CG feels he/she should be doing more for the PWD, and how often the CG feels he/she could do a better job for the PWD (Lawton et al., 2013).

\section{Evaluated Need Variable.}

Evaluated Need was measured by a combined variable including that the PWD has a health care provider, the health care provider recommended services, plus both formal and informal services received in hours per week.

\section{Non-Use of Services Measures}

Non-Use of Services (Dependent Variable). The dependent variable is support services not used by the CG. Formal (paid) and informal (unpaid) support services were analyzed as 14 
services (formal and informal combined) $(\alpha=.710)$, eight formal services individually, six informal services individually, and a dichotomous variable of users (not using 1-8 services) and non-users (not using 9-14) services. The outcome variable was calculated based on summing the eight Gitlin formal services and the six Caregiving Services Available informal services.

\section{The Final Survey/Questionnaire}

The survey took an average of approximately 30-45 minutes to complete the entire questionnaire. The time to complete the demographic information was approximately seven minutes, the Caregiver Appraisal Measure questions (Lawton et al., 1989; 2000) about one and a half minutes, the beliefs statements (Phillipson \& Jones, 2010; Phillipson et al., 2013) about 7 minutes, the Unmet Needs Assessment (Bass et al., 2003; 2012; 2013; 2014) approximately 10 to 15 minutes, questions from REACH I (Gitlin, Roth, \& Huang, 2014; Hughes, Lepore, Walberg, Gould, \& Walsh, 2018; Shulz, 2006) about 2 minutes, and additional questions about services approximately 3-5 minutes. Table 3 in Appendix E included a list of each variable and the instrument used for measurement for the proposed study. In order to assess comprehension and length of time to complete the survey, five former caregivers were asked to provide feedback regarding the survey/questionnaire. The five former caregivers reported understanding the survey and time to complete ranged from 17-26 minutes.

\section{Data Collection}

Data were collected via mailed or hand delivered surveys that participants could complete at home and returned by using a self-addressed United States postal envelope. For participants who needed assistance with completing the questionnaire, the researcher offered completion via telephone, or in person when an individual private area was available. The researcher identified potential participant dyads via the primary care provider offices, geriatric clinics, county 
agencies of aging, home health agencies, senior centers in Appalachian counties. There were 43 returned surveys, one completed in person in office by $\mathrm{CG}$, the rest returned via mail to the researcher.

\section{Data Analysis}

The collected data were coded and entered into SPSS v. 24 software as questionnaires were returned. Descriptive statistics as well as appropriate statistical testing for violation of assumptions occurred. The researcher performed data entry. Multiple linear stepwise regression was used to analyze variables for each research question, for the dependent variable, Non-Use of Services, calculations were completed for both (combined) formal and informal service non-use, formal service non-use, and informal service non-use. The ratio level outcome measure using stepwise linear regression was non-use of 14 services (combined both formal and informal) from Gitlin et al. (2003) and Caregiving Services Available, non-use of formal services, and non-use of informal services respectively. The categorical variable non-use of services (not using 1-8 were users, 9-14 were non-users) was used with logistic regression. All research questions were examined using both linear and logistic regression. Please see Table 8 in Appendix $\mathrm{N}$ for similarities and differences in the 14 services.

One hundred (100) questionnaire packets were delivered in person at caregiver support groups, geriatric clinics, area agencies on aging, and Home Health agencies. Forty-two were returned via US Postal Service and one was returned at the end of the physician visit. An additional 784 packets were provided to other sites and apparently not distributed for multiple reasons such as perceived complexity of the survey and limited time to interact with CGs. 


\section{Analysis of Research Questions}

1) What estimate of the variance in the non-use of services in Appalachian caregivers of persons with dementia can be explained by the predisposing characteristics of demographic variables, social variables, and belief (dementia) variables? Demographic variables CG gender and age were respectively categorical and continuous levels. Social variables included the CG level of education (ordinal level), Faith/Spiritual beliefs affecting important life decisions (categorical level), and the total score of the SPS 10 Revised (Russell et al., 1984), a ranking scale (ordinal level). Health (dementia) beliefs were measured by the total score of the Phillipson et al. (2013) 12 questions about the CG beliefs about dementia ranking scale (ordinal level). Services (dementia) beliefs were measured by the total score of the Phillipson et al. (2013) questions about services (dementia) and the CG ranking scale regarding the usefulness or potential harm of 12 services (ordinal level). Correlation analysis was used to determine the association between variables, namely the direction and strength of the relationship (Pallant, 2012). Reliability measured by Cronbach's alpha for SPS-10 R was 0.496; Beliefs Health (Dementia) was 0.449; and Beliefs Services (Dementia) was 0.657. The missing data in SPSS version 24 indicated was addressed by listwise deletion based on all variables in the procedure. Multiple linear stepwise regression was used to examine the predisposing variables in relation to the dependent variable non-use of both formal and informal (combined) services, non-use of formal services, and non-use of informal services. Logistic regression using block entry was used to examine the predisposing variables in relation to the dependent variable non-use of services, a dichotomous variable measured those CG who were not using 1-8 services were considered "users", and those CG not using 9-14 services were considered "non-users". 
2) What estimate of the variance in the non-use of services in Appalachian caregivers of persons with dementia can be explained by enabling factors of financial and organizational resource variables? Enabling financial was a combined variable from the level of income which was a rank scale (ordinal) and the UNALF total score of the subscale. Reliability for UNALF total subscale score $\alpha=0.811$. Enabling organizing was a combined variable of Total Caregiving Services Available and three total scores of subscales UNAFC, UNAES, and UNAFS. Reliability for Total Caregiving Services available was 0.680; UNAFC $\alpha=0.798$; UNAES $\alpha=$ 0.816 ; and UNAFS $\alpha=0.732$. The missing data in SPSS version 24 indicated was addressed by listwise deletion based on all variables in the procedure. Correlation analysis was used to determine the association between variables, namely the direction and strength of the relationship (Pallant, 2012). Multiple linear stepwise regression was used to examine the enabling financing variable and the enabling organizing variable in relation to the dependent variable non-use of both formal and informal (combined) services, non-use of formal services, and non-use of informal services. Logistic regression using block entry was used to examine the enabling financing and enabling organizing variables in relation to the dependent variable nonuse of services, a dichotomous variable measured those CG who were not using 1-8 services were considered "users", and those CG not using 9-14 services were considered "non-users".

3) What estimate of the variance in the non-use of services in Appalachia CGs of PWD can be explained by the need factors, both perceived and evaluated needs variables? Perceived need was a combined variable of the total score on the Caregiver Mastery subscale (Lawton, et al., 2013) with three subscale total scores for UNAHI, UNADLT, and UNALA (Bass et al., 2012). Reliability for Caregiver Mastery subscale Cronbach's alpha was 0.800; UNAHI $\alpha=$ 0.922; UNADT $\alpha=0.743$; UNALA $\alpha=0.903$; and for the combined perceived needs variable $\alpha$ 
$=0.928$. Evaluated need was a combined variable of having a health care provider, the health care provider recommending services for the PWD, plus both hours received weekly of both formal and informal services. The missing data in SPSS version 24 indicated was addressed by listwise deletion based on all variables in the procedure. Correlation analysis was used to determine the association between variables, namely the direction and strength of the relationship (Pallant, 2012). Multiple linear stepwise regression was used to examine the perceived need variable and the evaluated need variable in relation to the dependent variable non-use of both formal and informal (combined) services, non-use of formal services, and nonuse of informal services. Logistic regression using block entry was used to examine the perceived need and evaluated need variables in relation to the dependent variable non-use of services, a dichotomous variable measured those $\mathrm{CG}$ who were not using 1-8 services were considered "users", and those CG not using 9-14 services were considered "non-users".

4) What combination of predisposing, enabling, and need factors constitutes the strongest explanatory or associative model of non-use of services? Correlation analysis was used to determine the association between variables, namely the direction and strength of the relationship (Pallant, 2012). Multiple linear stepwise regression was used to examine the total score of the SPS 10-R variable (predisposing), total formal and informal caregiving services available, and the evaluated need variables in relation to the in relation to the dependent variable non-use of both formal and informal (combined) services, non-use of formal services, and nonuse of informal services. Logistic regression using block entry was used to examine the total score of the SPS 10-R variable (predisposing), total formal and informal caregiving services available, and the evaluated need variables in relation to the dependent variable non-use of 
services, a dichotomous variable measured those CG who were not using 1-8 services were considered "users", and those CG not using 9-14 services were considered "non-users".

\section{Human Rights and Ethics}

The researcher obtained WVU Institutional Review Board (IRB) approval. All eligible participants were provided informed consent and were able to stop participation at any time during the study, or decline to answer any questions, without penalties or repercussions. No persons who met eligibility criteria were excluded from the study based upon gender, lifestyle, religion, culture, or ethnicity. Mailed letters and recruitment emails to physician offices, area agencies on aging, how the data were to be used, and the methods ensuring the data were kept anonymous (separate locked areas for consent forms and numbered questionnaires; data destroyed after five years from close of study; data analyzed as group, not individually). Advertisements, flyers, and recruitment materials contained information about the purpose of the study, the voluntary participation, IRB approval number, whom to contact about the research, and the time required to participate to complete the study. For participants needing assistance to complete the questionnaire, the researcher offered in person completion with the respondents in two locations, and all respondents had access to an email account and phone number of the student researcher for any questions or concerns about the study. In locations with private rooms to maintain confidentiality, the student researcher aided the caregiver in person to complete the survey. No caregiver selected any of the options offered. The consent forms for each CG/CR dyad included the same information as the flyers, as well as information about methods to ensure anonymity, and the ability to skip questions if desired. The researcher did not expect the participants to incur any expense, as the questionnaire was mailed or handed to the CG/CR dyad at the caregiver association meeting, senior center, area agency on aging, or health provider 
office, and a pre-paid envelope were provided to return the completed survey. Participants were informed that they could take as much time as they needed. The researcher did not anticipate risks to the dignity, rights, health, or welfare of the human participants above those of everyday life. It may have been inconvenient to complete the questionnaire. No participants expressed any distress or strong emotional responses to any question content or reported experiences of psychological distress from memories evoked by the questions. The number for the Alzheimer's Association helpline at 1.800.272.3900 was provided as a resource, as well as the website URL http://www.alz.org/ (Alzheimer's Association, 2016). Additionally, the Area Agency on Aging national number 18006771116 was provided to give direction to local providers who may assist with developing a caregiving plan, specific issues related to the CG/CR dyad, and identify support services in the area (National Association of Area Agencies on Aging, 2015). Please see Appendix $\mathrm{N}$ for a list of national resources for caregivers, and a brief list of steps to take to seek services.

\section{Methods to Assure Rigor}

The researcher demonstrated rigor using psychometrically sound measurement tools, a representative sample of West Virginian caregivers, and scientific principles in research design and conduct. The validity of hypothesized relationships, called theoretical grounding, was one method used to demonstrate rigor in quantitative research design. Other methods to assure rigor were design validity, and ensuring statistical assumptions were met. Design validity describes threats to internal and external validity, including threats of history (intervening event), maturation (developmental change), testing (change cause by instrument), instrumentation (reliability of a measure), mortality (participant drop out), selection bias (poor sample selection), and low generalizability (external validity and unable to generalize findings) (Polit \& Beck, 
2014). The researcher ensured the instruments were psychometrically sound to assure design validity (Polit \& Beck, 2014). Rigor means to follow scientific techniques and strategies for increasing the trust and confidence in research findings. Correlational explanatory design was used to eliminate threats to history, maturation, and mortality as data were collected at one point in time. The use of reliable and valid instruments helped combat threats to testing and instrumentation. Recruitment of volunteer CG/CR dyads in West Virginia via caregiver support groups, geriatric clinics, primary care clinics, West Virginian county (and surrounding Appalachian counties) area agencies on aging, home health agencies, and senior centers helped selection of volunteer family CG of persons with dementia meeting eligibility criteria. Statistical assumptions included scaling, normal curve, linear relationships between variables, and test specific criterion.

\section{Summary}

In summary, chapter three explained the design and methods used to answer the four research questions regarding Appalachian caregivers of PWD. The researcher described the participants, quantitative research procedure, proposed measurements, and protection of human subjects. Outcomes of data collection included 43 mailed surveys, and analysis procedures using SPSS software were described. The next chapter includes the results. 


\section{Chapter 4 Results}

The factors most strongly associated with caregivers' non-use of formal and informal services in Appalachia (focused in WV and surrounding Appalachian counties) were identified from the 43 returned surveys. This chapter is organized in terms of the four specific research questions, guided by Andersen's model. The first three questions examined the estimate of variance in the non-use of services in Appalachia (focused in WV and surrounding counties) explained first by predisposing characteristics, then enabling factors, and finally need factors. The last question examines the combination of significant predisposing, enabling, and need factors constituting the strongest explanatory or associative model of non-use of services. Descriptive statistics, including means, standard deviations, and ranges were used to examine each variable as listed in Table 2: Selected Caregiver Measures of Service Non-use. Cronbach's alphas were calculated for all scales/instruments.

\section{Analysis of Scale Reliability}

Cronbach's alpha reliability estimates were computed for each multiple-item summated rating scale used in the study. In Appendix Q, descriptive statistics and internal consistency reliabilities were reported for scales and subscales. The SPS-10 scale had a Cronbach's alpha in the current study of 0.496 - below a desirable 0.70 reliability. After carefully evaluating the reverse coded item (questions 2, 3, 9, and 10; Cutrona \& Russell, 1987) no items were removed because scales with 10 or fewer items sometimes do not achieve recommended alphas (Pallant, 2012). These questions were also examined in relationship to the 170 -question total survey completed by participant caregivers. The mean inter-item correlation is 0.070 . Phillipson et al. (2013) Beliefs about Dementia Subscale Cronbach's alpha is 0.449, and Phillipson’s Beliefs 
about Services Subscale internal consistency is 0.657 (See Appendix Q). As noted above, these are lower than desired, and did not provide significant explanatory evidence.

The variables from Question \#2 from The Unmet Needs Assessment Legal Financial subscale Cronbach's alpha is 0.811 (see Appendix Q), had satisfactory internal consistency. Total Caregiving Services Available (both Formal and Informal) Cronbach's alpha was 0.680 for the thirteen items, approaching the desired 0.70. The Enabling Organizing questions from the three Unmet Needs Assessment sub-scale were strong Family Concerns, Emotional Support, and Finding Services had an internal consistency alpha of 0.848 .

For the four items in the Evaluated need subscale, there were too few responses to calculate a Cronbach's alpha. The Cronbach's alpha for Perceived Need sub-scales from the Unmet Needs Assessment of Health Information, Daily Tasks, and Living Arrangements was 0.928 for those 23 items (see Appendix Q). The internal consistency for the four item Perceived Need Total Caregiver Mastery sub-scale was 0.800, a strong Cronbach’s alpha. The linear regression outcome 14 item scale for non-use of both formal and informal services, consisting of eight formal care services from Gitlin et al. (2003), and six informal Caregiving Services, had an internal consistency of 0.710 (see Appendix Q).

\section{Regression Analyses}

\section{Dependent Variables.}

The dependent variable for linear regression used was 14 services, including eight formal (Gitlin) and six informal (Caregiving services) not used. The independent variables for each research question were entered into a stepwise regression for the 14 total formal and informal services not used, eight formal services not used, and six informal services not used to examine the robustness of the relationships in terms of formal versus informal services non-use. 
Both linear and logistic stepwise regression were conducted for all four questions (output available Appendix J, Tables 6.1 Q1A through 6.12 Q4C). The linear regression calculations used both formal and informal services ( $\mathrm{n}=14$ services) combined as the (ratio) dependent variable. Appendix O Table 9 describes the similarities and differences in the combined services (eight formal and six informal services). Additionally, to distinguish whether the formal services $(n=8)$ or informal services $(n=6)$ were not used, the eight formal services and the six informal services were individually examined by logistic regressions for all four questions. The logistic regression calculations outcome was a dichotomous variable using zero coding for "users" of services defined as those not using 1-8 (therefore using more than 8) services, and "non-users" were identified as those caregiver dyads not using 9-14 services (coded as 1). There were 17 users, and 23 Non-users of services.

\section{Predisposing Factors.}

The predisposing variables included demographic variables of CG gender (male or female); CG age in years; CG level of education (highest level of education completed less than high school, high school or GED, post high school, college degree, professional or graduate degree); CG relationship to the PWD (spouse or partner; adult child; sibling; friend; other); and faith or spiritual belief affecting important decisions.

Predisposing social variables were measured by the combined variable Social Provisions Scale Score- Short Version (Cutrona \& Russell, 1987) discussed above. Predisposing beliefs were measured by Beliefs about dementia and Beliefs about services for dementia (Phillipson et al., 2014; Phillipson et al., 2013) discussed above (Appendix D).

What amount of variance was supported by examination of predisposing factors was found to be the Social Support Scale (SPS) (Cutrona \& Russell, 1987) when using the 14 
combined services $(\mathrm{N}=40$, Adjusted $\mathrm{R}$ square $=0.083, \mathrm{~F}=4.550, \mathrm{p}=0.039)$. When selecting the eight formal services, no variables loaded. After using the six informal services as the dependent variable, the SPS scale was a predictor, but only for the six informal services $(\mathrm{N}=40$, Adjusted $\mathrm{R}$ square $=0.076, \mathrm{~F}=4.196, \mathrm{p}=0.047)$. The use of both formal and informal services was the most robust explanatory variable regarding predisposing factors for research question one.

One explanation for informal services not being used is that there are limited formal services in Appalachian counties (WV and surrounding counties). It may have to do with Appalachian cultural values, including strong family support systems, independent self-reliance, or distrust of structured health care systems (McGarvey, Leon-verdin, Killos, Guterbock, \& Cohn, 2011). Also, according to the ARC, socio-economic factors are worse for Appalachian counties compared to the rest of the United States (ARC, 2018).

Stepwise linear regression of the demographics, social, and belief variables on non-use of services was conducted to identify predisposing factor variables that best explained the estimate of variance. A logistic regression examined the same data using the dichotomous variable of user/non-user of services and there was no meaningful difference between the results. The Social Provisions Scale Revised-10 (SPS-10) explained 8.3\% to $10.7 \%$ of the estimate of variance for the dependent variable total (14) formal and informal services $\left(\mathrm{R}^{2}=0.107\right.$ and adjusted $\mathrm{R}^{2}=$ 0.083) (Table 6.1 Q1A in Appendix J). The only significant variable for logistic regression was also the SPS Scale Score $(\mathrm{p}=0.048)$. As the only significant predictor, the SPS Scale Score had an odds ratio of 0.728 , indicating those with higher social support were .728 times less likely to report not using a service. Basic descriptive statistics, correlations, and regression coefficients are shown in Tables 6.1 Q1A-6.12 Q4C Appendix J. 
To attain additional insight regarding the differences in non-use of formal services and informal services independently, stepwise multiple linear regression analyses were repeated on the outcomes of eight formal services and then on the six informal services. No variables loaded into the equation in Table 6.2 Q1B in Appendix $\mathrm{J}$ which examined the outcome of non-use of the eight formal services. Basic descriptive statistics and regression coefficients for the six informal services as shown in Table 6.3 Q1C in Appendix J identified the only significant explanatory predisposing characteristic variable was the Social Provisions Scale 10 Revised score $(p=.047)$. The model accounted for $13.4 \%$ of the variance in Non-use of Informal Services (Adjusted $\mathrm{R}^{2}=$ $0.076 ; \mathrm{F}=4.196, p=.047)$.

Based on these analyses in Appendix J (combined formal and informal services as the dependent variable), supported by Table 6Q1C (informal services as the dependent variable), the final regression analysis used the Total SPS score as the variable representative of a significant predisposing characteristic. Higher SPS scores indicate more perceived social support which explained higher non-use of services.

\section{Enabling Factors.}

The suggested explanatory variable for the combined services (formal and informal) regarding enabling factors were not as robust $(\mathrm{N}=38$, Adjusted $\mathrm{R}$ square $=0.117, \mathrm{~F}=5.894, \mathrm{p}=$ $.020)$ as the six informal caregiving services $(\mathrm{N}=38$, Adjusted $\mathrm{R}$ square $=0.137, \mathrm{~F}=6.878, \mathrm{p}=$ .013). The eight formal services did not load into the model. Enabling factors of total caregiving services (both formal and informal) predicted the non-use of the six informal services. Again, this may be because there are limited formal resources available in Appalachian counties in WV and surrounding states. Additionally, it may also be the culture of the area, as noted in Question 1 above. 
Stepwise linear regression of the financial and organizational variables on non-use of services was conducted to identify enabling factor variables that best explained the estimate of variance. A logistic regression examined the same data using the dichotomous variable of user/non-user of services and there was no meaningful difference between the results. The enabling financial variable consisted of the Unmet Needs Assessment Legal and Financial subscale, and household income; the enabling organizational variable included Total Caregiving Services Available, both formal and informal, and Unmet Needs Assessment sub-scales of Family Concerns, Emotional Support, and Finding Services. The linear stepwise regression used the outcome variable of 14 services (both formal and informal) Users/Non-users of service was the categorical variable for the logistic regression. The variable Caregiving Services Availability explained $11.7 \%-14.1 \%$ of the estimate of variance in the non-use of 14 services $\left(\mathrm{R}^{2}=0.14\right.$; adjusted $\mathrm{R}^{2}=0.117$ ) (see Table 6.4 Q2A in Appendix J). Logistic regression revealed no significant variables and no loadings.

Basic descriptive statistics and regression coefficients are shown in Table 6.4 Q2 A in Appendix J. The only significant explanatory enabling characteristic variable was the Total Caregiving Services Available (both formal and informal) $[p=.020]$. The linear regression results for Enabling variables with the dependent variable Formal (Table 6.5 Q2B in Appendix J) and the dependent variable informal services (Table 6.6 Q2C in Appendix J) are available.

In order to attain additional insight regarding the differences in non-use of formal services and informal services independently, stepwise multiple linear regression analyses were repeated on the outcomes of eight formal services and then on the six informal services. Nothing was significant on the outcome in the model with the dependent variable Non-use of eight Formal Services (Table 6.5 Q2B in Appendix J). Basic descriptive statistics and regression 
coefficients for the six informal services as shown in Table 6.5 Q2C in Appendix J, identify the only significant explanatory enabling characteristic variable was the total caregiving services available (both formal and informal) $[p=.020]$. The model accounted for $13.7 \%$ to $17.6 \%$ of the variance in Non-use of Informal Services $\left(\mathrm{R}^{2}=0.176 ; \mathrm{F}=2.382, p=.020\right.$; Adjusted $\mathrm{R} 2=$ $0.137)$.

Based on these analyses (combined formal and informal services as the dependent variable) and, supported by the findings in Table 6.5 Q2C in Appendix J (informal services as the dependent variable), the final regression analysis used the Total Caregiving Services Available (both formal and informal) score as the variable representing enabling characteristics. The fewer formal and informal caregiving services available explained higher non-use of services.

\section{Need Factors.}

After comparing the combined 14 formal and informal services, the eight formal services, and the six informal services, evaluated need of having a health care provider (HCP), HCP recommending services, paid hours help with volunteer hours help per week was the more robust predictor for the non-use of formal services. The output for the combined 14 services $(\mathrm{N}=42$, Adjusted $\mathrm{R}$ square $=0.162, \mathrm{~F}=2.456, \mathrm{p}=0.078$ ), the eight formal services of evaluated need were the hardier $(\mathrm{N}=42$, Adjusted $\mathrm{R}$ square $=0.255, \mathrm{~F}=15.004, \mathrm{p}=0.000)$. The factors did not load for the six informal services.

The reason for a more robust evaluated need and the formal services may be related to the HCP recommending the services. Perhaps having an HCP providing "permission" or an order for additional care creates a positive acceptance of the care. However, as noted regarding questions one and two, this would seem to contradict some aspects of Appalachian culture as discussed by 
McGarvey et al., 2011). Another reason for evaluated need and the eight formal services may be that the CG was unaware of how to access the services until the HCP recommended them.

Stepwise linear regression of the Evaluated and Perceived need variables on non-use of services was conducted to identify need factor variables that best explained the estimate of variance. A logistic regression examined the same data using the dichotomous variable of user/non-user of services and there was no meaningful difference between the results. Evaluated Need encompassed that the PWD had a primary care provider, that the health care provider recommended services, and included the total paid and unpaid hours of help/services used per week. Perceived need encompassed the Unmet Needs Assessment subscales of Health Information (HI), Daily Tasks (DT), and Living Arrangements (LA), and the total Caregiver Mastery sub-scale score. The linear stepwise regression used the outcome variable of 14 services (both formal and informal). Users/Non-users of service was the categorical variable for the logistic regression. Basic descriptive statistics and regression coefficients are shown in Table 6.7 Q3A in Appendix J. The only significant explanatory Need characteristic variable was Evaluated Need which explained $7.9 \%$ to $10.2 \%$ of the estimate of variance in the non-use of 14 services $\left(R^{2}=0.102\right.$; Adjusted $\left.R^{2} 0.079\right)$. The only significant variable for logistic regression was also Evaluated Need $(\mathrm{p}=0.029)$. As the only significant predictor, Evaluated Need had an odds ratio of 0.964 , indicating that those with higher evaluated need were .964 times less likely to report not using a service.

In order to attain additional insight regarding the differences in non-use of formal services and informal services independently, stepwise multiple linear regression analyses were repeated on the outcomes of eight formal services and then on the six informal services. Basic descriptive statistics and regression coefficients for the non-use of eight Formal services as 
shown in Table 6.8 Q3B in Appendix J identify that the only significant explanatory need characteristic variable was the Evaluated Need $[p=.002]$. The model accounted for $26.4 \%$ of the variance in Non-use of Formal Services (Adjusted R2 $=0.264 ; \mathrm{F}=5.861, \mathrm{p}=.002$ ). Nothing was significant on the outcome in the model with the dependent variable Non-use of six informal Services (Table 6.9 Q3C in Appendix J). Based on these analyses in Table 6.7 Q3A (combined formal and informal services as the dependent variable) and, supported by Table 6.8 Q3B (formal services as the dependent variable), the final regression analysis used the Evaluated Need score as the variable representing need characteristics. If the Health care provider recommended the use of services, there was a lower non-use of formal services.

\section{Combination of Predisposing, Enabling, and Need Factors Explaining the Model of} Non-Use of Services.

To address Question \#4, what combination of predisposing, enabling, and need factors constitutes the strongest explanatory or associative model of non-use of services, the findings of the first three research questions were entered into a linear regression equation. The three significant predictors included the "SPS-10 Revised scale score" as the predisposing factor, the "availability of services" (both formal and informal) as the enabling factor, and "evaluated need" as the need factor that were entered into the regression analyses. The linear stepwise regression dependent variable was 14 formal and informal services not used. A logistic regression examined the same data using the dichotomous variable of user/non-user of services and the logistic model outcome did not include the predisposing factor (SPS scale score) but was consistent with the explanatory roles of Caregiving Services Available and Evaluated Need factors.

The SPS scale dropped out of the model for the eight formal and six informal services. The model outcomes for question four regarding the strongest predictor for combined formal and 
informal $(\mathrm{N}=42$, Adjusted $\mathrm{R}$ square $=0.353, \mathrm{~F}=12.197$, and $\mathrm{p}=0.002)$, and a Beta weight for total caregiving services available of 0.210 indicating this is $80 \%$ trustworthy. The strongest two predictors of non-use of services accounted for $35.3 \%$ of the variance (Adjusted $\mathrm{R}$ square $=$ $0.259, \mathrm{~F}=15.350$, and $\mathrm{p}=0.000)$ for evaluated need was the most robust predictor, and total caregiving services available was the second most robust predictor. The six informal services $(\mathrm{N}=42$, Adjusted $\mathrm{R}$ square $=0.169, \mathrm{~F}=9.364$, and $\mathrm{p}=0.004)$ with the total caregiving services available.

This outcome supports the importance of the HCP recommending services and the availability of the services. Logically, accessing services was influenced by the availability of services, and the HCP recommendations appear to be the strongest predictor of lower non-use of services.

A forced entry linear regression analysis based on the theoretical model suggested that the Total SPS Scale score, Caregiving Services Available score, and Evaluated Need score together would explain $40 \%$ of the variance in non-use of services. However, the low reliability of the SPS scale score, as well as the inconsistency with the logistic regression analysis, decreased confidence in this result. A multiple linear regression stepwise analysis was then used to identify the strongest explanatory model of non-use of services (both formal and informal), using the variables of the Total SPS Scale score, Caregiving Services Available score, and Evaluated Need score. Basic descriptive statistics and regression coefficients are shown in Table 6.10.Q4A through 6.12 Q4C in Appendix J. Each predictor variable had a significant $(p<.05)$ zero-order correlation with non-use of both formal and informal services, but only Caregiving Services Available and Evaluated Need variables had significance $(p<.05)$ in the full model. The two-predictor model was able to account for $35.3 \%$ of the variance in the outcome which 
measured a participant's non-use of both formal and informal services (Adjusted $R^{2}=.353, F=$ $12.20, p<.001)$

In order to attain additional insight regarding the differences in non-use of formal services and informal services independently, stepwise multiple linear regression analyses were repeated on the outcomes of eight formal services and then on the six informal services. Basic descriptive statistics and regression coefficients examining non-use of formal services are shown in Table 6.11 Q4B in Appendix J. Evaluated Need accounted for $25.9 \%$ of the variance in Nonuse of Formal Services (Adjusted R2 $=0.259 ; \mathrm{F}=15.350, \mathrm{p}=.000$ ). If the Health care provider recommended the use of services, there was a lower non-use of formal services.

Basic descriptive statistics and regression coefficients examining non-use of informal services are shown in Table 6.12 Q4C in Appendix J. Availability of Services (both formal and informal) accounted for $16.9 \%$ of the variance in Non-use of Informal Services (Adjusted R2 = 0.169; $\mathrm{F}=9.364, \mathrm{p}=.004)$. If Caregiving Services were available, there was a lower non-use of informal services. According to Pallant (2012), when variables are highly correlated, there is a risk of collinearity which can mask the true relationship between variables by inflating or trending measures toward the null value. In the case with highly correlated variables, a variance inflation factor (VIF) is recommended to examine the possibility of (multi)collinearity with a value above 10 suggesting the need to adjust for multicollinearity. In research question four, the variables were evaluated and with no VIF was found to exceed 10. While there were correlational relationships between variables, multicollinearity was not supported in the models.

\section{Summary}

The results of the analyses for each of the four research questions explained the estimate of the variances in Non-use of services by Appalachian caregivers of persons with dementia 
guided by Andersen's Behavioral Model. The significant predisposing factor was social beliefs, the significant enabling factor was caregiving services availability, and the significant need factor was evaluated need. When the three factors/variables were entered together based on the theoretical model into a linear regression, they together explained $40 \%$ of the variance in non-use of services. However, when a stepwise linear regression was used, the social beliefs variable was removed due to lack of significance, leaving caregiving services availability and evaluated need explaining $35.3 \%$ of the variance in non-use of services. The results indicate that not having support services available, and not having a primary care provider who recommends services including paid and unpaid help weekly were the strongest explanatory model for the non-use of services by caregivers of persons with dementia. 


\section{Chapter 5}

This chapter presents a summary of the study and conclusions drawn from the data presented in Chapter 4. Chapter five provides a discussion of the implications for action and recommendations for further study.

\section{Summary of Study}

\section{Overview of Problem.}

Caregivers of Persons with Dementia do not use support services to assist with caregiving, despite multiple research studies indicating there are interventions improving the CG and PWD lives, decreasing admission to long term care facilities, and helping to decrease stress and burden on the CG. The non-use of services may be worsened in an Appalachian setting, where there are fewer people in the population, a higher concentration of older adults compared to younger adults, fewer services, transportation difficulties, and long-distance travel may be required to use any of the specialty health services.

\section{Review Purpose statement.}

The purpose of the study was to determine factors that contribute to Non-use of services by CG for PWD. The Anderson Behavioral Model of Service Use guided the study, employing variables that predispose, enable, and identify needs contributing to the use or Non-use of services. The first three research questions identified predisposing, enabling, and need factors that explained the estimated variance in the model. The fourth question identified the strongest factors that together resulted in the Non-use of services. 


\section{Review of the methodology.}

The study employed a correlational explanatory design (Frankel \& Wallen, 2000). A mailed or hand-delivered survey was used to obtain demographic, predisposing, enabling, and need factors about CG of PWD and Non-use of services. These data can then be used to design interventions to address the needs of CGs of PWD in Appalachia. The researcher used IRB approved flyers, newsletter ads, agency contact emails, or phone calls, and individual agency approved advertisement methods (invitation to group meetings, dinners, etc.) to notify eligible caregiver dyads about the research study. In-person distribution of questionnaires was also completed in two geriatrician offices (one medical, one psychiatric), with the participants returning the surveys in a self-addressed postage paid envelope or completing on site and leaving with a staff member to place in mail. The rationale of a multiple method distributed survey was to collect information from individuals about the non-use of services, allow time and privacy to complete the questionnaire, avoid bias of "expected answer" if researcher was in room awaiting survey hardcopy. The researcher collected data on the availability and use of services within the CG/CR community (See Appendix I).

\section{Review Research Questions.}

1) What estimate of the variance in non-use of services in Appalachia can be explained by the predisposing characteristic of demographic, social, and belief variables?

2) What estimate of the variance in non-use of services in Appalachia can be explained by the enabling factors of financial and organizational resource variables?

3) What estimate of the variance in non-use of services in Appalachia can be explained by the need factors, both perceived and evaluated? 
4) What combination of predisposing, enabling, and need factors constitutes the strongest explanatory or associative model of non-use of services?

\section{Review of Major Findings.}

The most significant predisposing factor was social support, measured by the Social Provisions Scale Short (SPS-10 R) Version (Cutrona \& Russell, 1987; Russell et al., 1984), although a low reliability for the SPS-10R may have been the reason this factor dropped from the

final explanatory model. The enabling organizing factor that was most robust was the availability of caregiving services, both formal and informal. The most significant need factor contributing to non-use of services was evaluated need. The combination of the three factors explained $40 \%$ of the variance in the model of non-use of services. The most robust variable in the model was enabling organizing, availability of caregiving services (both formal and informal), and the the second strongest was evaluated need (having health care provider [HCP], $\mathrm{HCP}$ recommending services, and total weekly hours of formal and informal help received per week); the third variable of significance in research question one but not in the final model was social support (SPS 10R; from demographic, social, and belief variables).

\section{Review of Additional Findings.}

The survey used in the research study had space for respondents to write comments about caregiving and using services and asked if they wanted to expand on something "missing" from the questionnaire. Twenty- four out of 43 caregiver respondents included comments. A focused content analysis identified twelve topics contributed by CGs (See Table 10 Appendix P, Focused Content Analysis Respondent Comments). The twelve topics included: "Don't need additional services/don't need services yet"; "Not Qualifying/Not eligible for services"; "Lack of Knowledge of existing services"; "No stranger in home"; "Too much information too soon 
(overwhelmed)"; "Needs help accessing resources"; " Opposition/Struggle between CG/PWD about need/refusal of services (Hard for PWD to accept help or PWD may deny need for help)"; "Wanting permission to seek more resources/support help"; " Navigation", "Need for financial resources"; "Feeling family obligation to provide care"; and "CG lacks trust someone can do as well as he/she can". Research supported these themes and was included in Table 10 Appendix P.

\section{Discussion}

The purpose of the current study was to estimate the variance in non-use of services using Andersen's model of predisposing, enabling, and need factors. The following section discusses the findings in relation to the literature.

\section{Predisposing.}

\section{Demographic and Social.}

The current study did not identify any of the demographic variables (CG age and gender) explanatory in variance of non-use of services. Similarly, Phillipson et al. (2013) also reported no predisposing demographic variables to be associated with non-use of day respite or residential respite services. Robinson et al. (2013) reported non-users of services lived with the PWD, were spouses, older, reported more depression and received less social support. The current study finding that more social support explained higher non-use of services.

On the contrary, Sun et al. (2008) reported males were more likely to use more in-home services, while females used more transportation. Ploeg et al. (2009) reported demographic variable of age and social variable level of education were significant in understanding CG use of services. Martindale-Adams, Nichols, Zuber, Burns, and Graney (2016) described CGs who were users of services to be older, Caucasian verses Latino, married, and a spouse to the PWD, and CG had a higher level of education. Vecchio, Fitzgerald, Radford, and Fisher (2016) also 
indicated positive and significant relationships for using services (domestic assistance, centerbased care, and respite service hours).

Data from this study indicate more social support explained higher non-use of services. Likewise, Robinson et al. (2005) found social support affected use of services. However, Robinson et al. (2005) reported the spousal CG relationship to PWD was associated with non-use of services, which was not supported by the findings of this study. Differing from the current study results, Robinson et al. (2013) reported there was an association with use of services and the CG relationship to the PWD, and younger CGs were more likely to use assistance and respite. The current study does not support these findings. Another finding in the research literature by Brandảo, Ribeiro, and Martin (2016) reported non-users of residential respite services (day centers or home nursing care) reported a lower level of education, while level of education was not a significant factor in this study.

\section{Health Beliefs (Dementia) and Health Beliefs Services (Dementia).}

The current study identified no health beliefs (dementia) or health beliefs services (dementia) to be statistically significant in explaining the variance in non-use of services. The findings are partially consistent with Phillipson et al. (2013) regarding predisposing health beliefs. In contrast, Phillipson et al. (2013) identified negative service beliefs (dementia) as the strongest correlates with the non-use of respite services in-home. If a CG believed there would be a negative outcome for the PWD, there was a strong association with non-use of day care and residential care (Phillipson et al., 2013). 


\section{Enabling.}

\section{Financial.}

Enabling financial factors were not significant in the model in this research study.

Conversely, Brown, Friedemann, and Mauro (2014) stated lower CG income was associated with use of adult day services as compared to CGs with higher income. Brandảo, et al. (2016) reported non-users of residential respite services (day centers or home nursing care) reported a lower income. The findings in the current study were unexpected, as literature from the Resources for Enhancing Caregiver's Health (REACH I) study indicated the CG absorbs much of financial costs for the PWD care, $\$ 23,436$ for informal care and $\$ 8,064$ for formal services (Harrow et al., 2004). Martindale-Adams et al. (2016) described CG who used services had a higher income and were more likely to be retired. Vecchio et al. (2016) described one PWD enabling characteristic as receiving a government pension.

\section{Organizing.}

Findings from the research study indicated organizing resources explained the greatest estimate of variance of enabling factors in the non-use of services, in that the fewer formal and informal caregiving services available, the higher non-use of services was explained. Phillipson et al., (2013) identified enabling organizing to be "personal and community resources" (p. 416). Phillipson and colleagues asserted if CG did not have someone to assist them in finding services to help with the caregiving role, they were more likely to be non-users of day centers for respite. Ploeg et al. (2009) reported both formal and informal support were significant in understanding CG use of services. O’Connell, Hawkins, Ostaszkiewicz, and Millar (2012) identified CG who were employed outside the home were more likely to use respite services. Vecchio et al. (2016) identified PWD enabling characteristics to include availability of informal support (i.e. living 
arrangements) and geography. Dizzazo-Miller, Pociask, and Samuel (2013) identified when

PWD were in initial stages of dementia, the most used resource was the Alzheimer's'

Association training (unclear if a course or group), but CG reported distance and travel issues were barriers to using services.

\section{Need.}

\section{Evaluated.}

The combination variable for evaluated need included the following: PWD had health care provider (HCP); HCP recommended services; and total weekly hours of formal and informal help received per week. Evaluated need was the strongest explanatory variable for nonuse of services in this study. Conversely, Phillipson et al., (2013) reported no need factors were significantly associated with non-use of day centers. In Australia, Harrison, Low, Barnett, Gresham, and Brodaty (2014) identified that CGs of PWDs receive a "community care package" provided by the government, did not match with their needs, primarily in social and recreational activities, eating, and both mental and physical health. Jarott, Zarit, Stephens, Townsend, and Greene (2005) described a significant outcome when formal care hours increased, which was a negative association with indicators of CG stress (caregiver stressors decreased with the addition of formal care hours). Liu, Almeida, Rovine, and Zarit (2018) reported adult day service use had a positive impact on CG health as indicated by levels of cortisol (stress measure). In the qualitative study by Forbes et al., (2015), reported CGs identified difficulties obtaining resources for HCPs when counseling CGs, and there were challenges with communication and difficult relationships with physicians. The qualitative study by Heinrich, Laporte Uribe, Wubbeler, Hoffman, and Roes (2016), listed CG needs to obtain dementia-specific information through dementia care networks. DiZazzo-Miller et al. (2013), identified in the late stage of dementia, the 
most commonly used support resource was self, or family and the least valuable resource was the physician. Findings from a phenomenological study (DiZazzo-Miller et al., 2013) suggested in the early stage of dementia, CGs preferred referrals from health care professionals in relation to appropriate support services. Górska et al., (2013) found in a qualitative study most CGs were satisfied with services available and used, but there were still many unmet needs. CGs in the study (Górska et al., 2013) identified obstacles to obtain a diagnosis, dismissal of concerns and symptoms reported by family members, and a perceived lack of communication between all services involved in supportive care services. Other barriers reported include poor coordination of services after diagnosis of dementia, and a lack of care continuity (same people involved caring service), and problems with access to non-pharmacological interventions (Górska et al., 2013). Non-pharmacological interventions included access to therapies, such as occupational, speech and language, physical activities, day services, psychological services, and social engagement (Górska et al., 2013).

\section{Perceived.}

The combined perceived need variable included the Unmet Needs Assessment (Bass et al., 2003; 2012; 2014) subscales of Health Information (UNAHI), Daily Tasks (UNADT), and Living Arrangements (UNALA), plus the total Caregiver Mastery Subscale Revised (Lawton et al., 1989; 2013). The Perceived Need variable did not load into the model, suggesting multicollinearity among the independent variables, although further testing did not support multicollinearity (Pallant, 2015). On the contrary, Phillipson et al. (2013), stated the needs of the PWD (such as assistance with ADLs) were associated with non-use of respite services; those PWD who required no help with ADLs and were less cognitively impaired, were more likely to 
not use the service. During the middle stage of dementia, CGs reported internet resources were most helpful in finding health (dementia) information (DiZazzo-Miller et al., 2013).

\section{Services Not Used.}

The results of this study did not overwhelmingly indicate more formal or informal services were not used. Predisposing variables in research question one and enabling variables in research question two indicated informal services were not used the most. However, for question three, formal services were not used the most - this may relate to the importance of a health care provider recommending the use of formal services. Further studies should seek to evaluate the number of formal and informal services available, and which ones are used or not used. One method of ascertaining the information is to conduct a survey of available resources (formal and informal) in each county in Appalachia. The researcher used the Alzheimer's Association website community resource locator (2018) for adult day services near the 25541-zip code in West Virginia. Of the first 10 listed services, one was eliminated due to distance. The other nine were called. Seven had phone numbers "no longer in service", one number accepted recorded messages, but no one returned two calls in two weeks. The only one number answered indicated "we have not had adult day services for over two or three years" (Personal Communication, August 5, 2018). The employee of the agency indicated "there is the F. A. I. R. program (Family Alzheimer's In-home Respite), but it [has a] long wait list, is income dependent, and provides home health." The researcher contacted the general number of the Alzheimer's Association website 1-800-272-3900 and notified two different operators of the incorrect information. One provided more numbers of the "firm that has managed our resource contact information for over five years". Researchers may need to conduct a physical surveillance of each county, or work with the Area Agency on Aging contacts in each county to obtain accurate information. 


\section{Conclusions}

By encouraging the 16 million CGs of PWDs to sustain emotional, physical, and social well-being, PWD may live longer in the community and avoid costly long-term care (Alzheimer's' Association, 2018). The community must address access to quality dementia care services, assist with financial barriers, and provide person-centered appropriate care for CGs and PWDs. The current study identified enabling organizing, evaluated need, and social support as explanatory factors in non-use of services by CGs of PWD in Appalachia.

Although Andersen's model is useful to study non-use of services by CG of PWD, there are some limitations in identifying the best measures of the variables. The researcher used a substructed model of Andersen's Model of Behavioral Health, guided in part by Phillipson et al., (2013). The factors identified in the literature as predisposing, enabling, and need factors vary in category and measurement by researcher, and caregiving situation. For example, in a secondary analysis survey using Andersen's Behavioral Model of Health Services Use, enabling factors included income and availability of services, comparable to this study, but also included insurance status (Brown et al., 2014). Need factors in the current study included evaluated and perceived need, both combination variables, including having a $\mathrm{HCP}$, a $\mathrm{HCP}$ recommending services, and total hours services used per week for enabling need. Perceived need included the UNAHI (health information), UNADT (daily tasks), and UNALA (living arrangements) combined with the total score of Caregiver Mastery Subscale (how caregiver feels about caregiving experience). In contrast, Brown et al. (2014) included measures of level of illness, emotional distress, functional ability, cognitive status, CG perceived need for adult day services, and CG depression. While this study and Brown et al., (2014) categorized social support as a predisposing variable, Martindale-Adams et al. (2016) considered social support to be an 
enabling variable. Potter (2018) described how being a member of an ethnic or racial group may be both predisposing and enabling, because members of groups may have beliefs predisposing them to non-use of services, while there may also be an ethnic/racial barrier when trying to access services, such as language or acceptance of help (Parveen, Peltier, \& Oyebode, 2017). The variables from Andersen's Model for this study are discussed from the strongest explanatory factor in the model, enabling organizing, to the weakest, predisposing social support.

\section{Enabling Organizing Total Caregiving Services Available.}

Having both formal and informal caregiving services available is the first step in providing support to CGs of PWD. Findings from the study supporting available caregiving services was expected, although what was slightly unexpected was that available caregiving services was the strongest explanation of variance in the non-use of services. The services would need to address family concerns, provide emotional support, and be well-known in the community to help CGs of PWD. Phillipson et al. (2013) also reported enabling factors of the CG not having someone to assist them to find services was associated with non-use of respite services. In reviewing the additional findings from the respondent comments, the themes "lack of knowledge of existing services", "needs help accessing resources", "wanting permission to seek more resources/support help", and "navigation" may relate to enabling organizing. The study suggests that not knowing about services that are available, wanting someone to help identify (provide knowledge) available formal and informal services, providing emotional support for a CG to give himself or herself permission to access the service, and providing assistance to navigate available resources could decrease the non-use of services. The idea of one central person to coordinate community resources, the health care team, volunteer services, and communicate with the family is supported by Reuben et al. (2013), who used a Dementia Care 
Manager (a nurse practitioner). Using a nurse practitioner to coordinate with all stakeholders and manage medications may be ideal. One potential detractor to this service would be the cost of implementing Dementia Care Navigators to the health care system. An argument can be made that the initial costs may be money-saving in the long term, reducing hospitalizations and longterm care placement of PWDs. Additionally, having a team of a nurse practitioner, nurse, and social worker may be ideal as a regional resource. The team would be able to provide in-home needs assessments (nurse) to personalize a plan of care for each CG/PWD dyad, the social worker may identify appropriate resource referrals, and the NP can communicate with all the stakeholders, manage physical care, and work with the team to form one point of access for health care professionals, community and volunteer resources, and families. Also, the team could market their program and services, involve community and faith-based organizations as liaisons to $\mathrm{CG} / \mathrm{PWD}$ in community, and offer professional education for health care professionals. Again, the initial cost would be concerning, but the overall cost savings to prevent unnecessary hospitalizations and ER visits for the CG and PWD, as well as avoid or prolong long term care placement of the PWD, would justify the initial expense.

Another way to provide support for CGs and PWD is for academic institutions, community services, faith-based organizations, and health care facilities to work together and provide CG training and emotional support, while at the same time offering care and activities for the PWD (perhaps a Community Academic Family Faith Health Organization [CAFFHO]). Faith-based institutions and community volunteers may partner to provide facilities and volunteers to assist in offering one or two four-hour sessions weekly while faculty supervised health professions students (nursing, medicine, pharmacy, social work, psychology, physical therapy, nutrition, dentistry, occupational therapy, etc.) attend scheduled community respite care 
centers as part of clinical rotations. These respite sites could offer health care monitoring; classes for both caregivers and persons with dementia; occupational, nutritional, and physical services; health insurance expertise to assist with health care coordination; emotional support on site; and respite care provided by supervised students a certain number of hours per week. The cost would need to be income-adjusted. Health care professionals associated with the health care facilities (local medical centers including VA Medical Centers), academic institutions (West Virginia University, Marshall University, and other institutions of higher learning), and community services (Alzheimer's Association, Area Agencies on Aging, American Association of Retired Persons [AARP], and the West Virginia Bureau of Senior Services Family Alzheimer's InHome Respite [FAIR]), could provide evidence-based multicomponent interventions for CGs and PWDs, tailored to CGs who are caring for PWD across the stages of dementia (early, middle, late). In early dementia, while a PWD may not need ADL/IADL support, receiving education about dementia (progression, online sources of information, and support to help cope with the impact of dementia, including legal and financial issues, cessation of driving, etc.) and alternative therapies (yoga, exercise, music and art therapies, meditation) to help develop coping and relaxation techniques may be useful (Whitlatch \& Orsulic-Jeras, 2018). Evidence-based programs such as Support, Health, Activities, Resource, and Education (SHARE) have been useful to identify and explain PWD/CG values and preferences, develop a person-centered plan of care, and provide a base for future decision making (Whitlatch \& Orsulic-Jeras, 2018, p. S63).

During the middle stage of dementia, information/educational support personalized to the type of dementia affecting the CG/PWD dyad is warranted. The behavioral and psychological symptoms (BPSD) of dementia and Alzheimer's Dementia and related dementias (ADRD) become more frequent and obvious during the middle stages (Whitlatch \& Orsulic-Jeras, 2018). 
The exception is Frontotemporal Dementia, in which early symptoms are personality and behavioral changes (Morhardt, 2011). During the middle-stage of caring for a PWD, the CG needs help dealing with unwelcome behaviors by the PWD, emotional support to reduce isolation and grief, and perhaps professional help coping with the changes (Reed, 2012). The CG may start using respite care, home health, or some assistance with household duties to care for himself or herself. Other concerns the CG in the middle-stage may find difficult are environmental safety for the PWD living at home, medical care, and planning future living arrangements. Unfortunately, during the late-stage of caregiving, the CG has financial and legal responsibilities, and consider long-term-care placement, or Hospice services (Reed, 2012).

There are evidence-based programs such as Advanced Caregiver Training (ACT) and Caregiver Skill Building (CSB) that help with recognizing, managing, and coping with BPSD (Whitlatch \& Orsulic-Jeras, 2018), which may be offered for CG/PWD dyads attending the CAFFHO. At this phase, the PWD may need supervised activities (exercise, education, support) while the CG might attend a CG program and support group, and then the dyad may come together for a snack or meal. Evidence-based caregiver programs include: The Benjamin Rose Institute Care Consultation (BRI-CC) (Bass et al., 2014); CarePRO Care Partners REACHING OUT (Coon et al., 2016); Powerful Tools for Caregivers (Kuhn, Hollinger-Smith, Presser, Civian, \& Batsch, 2008); New York University Caregiver Intervention (NYU-CI) (Gaugler, Roth, Haley, \& Mittelman, 2008; Gaugler, Mittelman, Hepburn, \& Newcomer, 2010). The BRICC (Bass et al., 2014) offers phone coaching and support. CarePRO Care Partners REACHING OUT (Coon et al., 2016) supports education, skills training, communication, and promotes selfcare. Powerful Tools for Caregivers (Kuhn et al., 2008) helps CGs maintain own health and manage stress, communicate effectively with family members and service providers, and manage 
challenging emotions. The NYU-CI (Gaugler et al., 2008; Gaugler et al., 2010) is a multicomponent intervention program for CGs and PWD supplying education, service referrals, counseling, and management of stress. There are also programs through the Alzheimer's' Association, Family Caregiver Alliance, and Rosalyn Carter Institute for Caregiving. Other multicomponent interventions include Care of Persons with Dementia in Their Environment (COPE) (Gitlin et al., 2010), and Resources for Enhancing Alzheimer's Caregiver Health (REACH II) (Belle et al., 2006; Lykens, Moayad, Biswas, Reyes-Ortiz, \& Singh, 2014). For the later stage of dementia, discussion of placement in care facility, end-of-life-care, and/or palliative care may be offered (Whitlatch \& Orsulic-Jeras, 2018).

\section{Team Approach.}

The next step would be to develop a Dementia Liaison Team of a nurse practitioner (NP), registered nurse (RN), and master's prepared social worker (MSW) working with a physician as a regional resource. Adding a psychologist may offer the team insight for approaches to certain CG/PWD barriers to accepting care, at least in the beginning. The team RN would be able to offer in-home needs assessments to personalize a plan of care for each CG/PWD dyad. The MSW would find and ease appropriate resource referrals. The NP would be the point of contact for the team to manage physical care, with one point of access for health care professionals, community and volunteer resources, and families. The NP would communicate any care delivered and/or recommended for the CG/PWD dyad to the primary health care provider, and the rest of the involved services. The physician would be able to give input, recommendations of care, and act as a resource for the Dementia Liaison Team as needed. Also, the team could market their program and services, involve community and faith-based organizations as liaisons to CG/PWD in a community, and offer professional education for 
health care professionals. The Dementia Liaison Team may need expansion to offer a one-stop shop availability during health care provider appointments. A team member presence in the office during certain times, or via telecommunications from the health care provider office. The idea would be the health care provider might go with the CG/PWD dyad to the team office (or via a private Skype) to introduce the dyad to the Dementia Liaison Team. The program could be based on the findings of this study to decrease non-use of services by conducting research addressing service availability and health care provider recommendation of services.

\section{Evaluated Need (HCP Recommends Services).}

In the study, evaluated need was a combined variable including having an HCP, the HCP recommending services, and the total weekly hours formal and informal support received per week. HCP recommending service use was the second strongest explanatory variable of variance in non-use of services. The health care provider needs to be able to recognize symptoms of dementia, assess individual's needs, and make proper referrals. The findings are not unexpected, as one study showed the first choice for help in caregiving is the physician, especially if the CG is female and has a higher level of education (Ploeg et al., 2009). Brandảo et al. (2016), found most CGs learned about available services for the PWD from a health professional, mostly nurses. DiZazzo-Miller et al. (2013), described CGs as wanting proper service referrals from the HCP in the early stage of dementia, but in the late stage of dementia, specified the least valuable resource as the physician. Forbes et al. (2015) described differing results, reporting CGs indicated difficulties obtaining resource referrals from physicians, communicating, and had strenuous relationships with physicians.

Clearly, all HCPs (physicians, nurses, social workers, psychologists, etc.) should be alert to an individual's need for care and recognize CGs as at risk for negative health effects of the 
caregiving role. As part of the health care system, nurses could recognize CG burnout and suggest the PWD may need more help than the CG is able to provide. The approach should be individualized to provide care at a level needed by the PWD. An important skill for the nurse is assessment of the PWD and CG to identify needs and help with developing a plan of care. Nurses could promote the benefits of adult day services for the PWD in addition to the CG and address any negative service beliefs (Phillipson \& Jones, 2012).

The recommendation from the physician/clinician to the CG to use services may relate to "granting permission" as identified in the focused qualitative review. CGs perceive the physician recommendation as an order or prescription, rather than abdicating responsibility of providing care, or a way to ease guilt. Some CGs have an incredible resistance to using services to assist in the caregiving role. The physician referral to services may allow the CG to see obtaining help as "following orders" to improve the caregiving condition. Petersen, Hahn, Lee, Madison, and Atri (2016) reported CGs anticipate the primary care provider will suggest, approve, and direct them to specific support services that meet the individual CG/PWD dyad needs.

One reason CG/PWD dyads endure unmet needs is the lack of dementia specific education for health care professionals in primary care. Research indentifies "significant effects" when educational translational programs were implemented; there were positive outcomes for health care providers knowledge and attitudes about dementia, a more person-centered approach to care, and a constructive outcome on the clinical practice of dementia care (Wang, Xiao, Ullah, He, \& De Bellis, 2017, p.1). Turner, Eccles, Elvish, Simpson, and Keady (2017) indicated the same lack of knowledge and comprehension about dementia among health care staff in general hospital settings. The outcomes of the knowledge deficit include poor staff confidence in 
providing communication and managing undesirable behaviors of PWD, and negative attitudes towards PWD, and incompetence delivering person-centered care (Turner, 2017).

Weiss et al. (2017) found gaps in dementia education and training for health care professionals and informal caregivers. Recommendations by the researchers were to identify evidence-based training to create a dementia capable workforce; recognize minimal proficiencies required by health care providers; and for institutions of higher learning, health care professional accrediting bodies, licensing boards, federal agencies, and health care professional organizations to advance dementia care competencies (Weiss et al., 2017). Dementia capable workforce includes members of health care professions students, faculty, clinicians, assistive medical staff, and PWD/CG and families (Weiss et al.).

Despite the United States health care system relying on family CGs to provide $80 \%$ of care for PWD, CGs are overlooked and unrecognized in the planning of care and policy (Gitlin \& Hodgsen, 2016). The majority of CGs are thrust into a role for which they are unprepared, and at the same time are losing pieces of a loved one, who is also experiencing changes in role, function, and capabilities, while both CG and PWD are experiencing grief. CGs handle maintaining the PWD safety, health (both psychological and physical), and activities of daily living. However, CGs have little input into the current health or social systems affecting themselves and their loved ones with dementia.

Education of health care professionals is needed to identify caregivers and community resources. One method to help HCPs is to provide one or two community contacts for sources of support. The HCP may choose to offer a "geriatric" day in the practice, offering CG classes, respite care during the class for the PWD, and emotional support providers (both professional and community members) available to assist with positive coping and relaxation techniques. The 
"geriatric day" may include community resource contacts providing information about support services individually to the CG, naming services the CG and PWD may need. Ideally, CGs would be able to contact one person or group to manage the navigation to appropriate support services. Primary care providers can periodically screen CGs for complications of caregiving such as depression, stress, and anxiety, but assessment tools and referral networks should be in place to help the already busy provider with readily available measures and a one-point referral to care access for further needs assessment.

\section{Opportunities for Nurses in Primary Care.}

According to researchers, approximately $50-80 \%$ of persons with dementia are not diagnosed or misdiagnosed in the primary care setting, even in countries with advanced medical care systems (Cordell et al., 2013; Eichler, Thyrian, \& Hoffman, 2014; Eichler et al., 2015; Pathak \& Montgomery, 2015; \& Valcour, Masaki, Curb, \& Blanchette, 2000). Weiss et al. (2017) identified a gap in the interprofessional education of health care professionals about dementia care coordination and management. Study findings supported using a two-stage system when screening to help early detection of cognitive impairment (Grober, Wakefield, Ehrlich, Mabie, \& Lipton, 2017). Eichler et al. (2015) suggested when screening for dementia in primary care, more diagnostic tests be mandatory because of a high number of false positive screening results. Cordell and colleagues (2013) recommended using a structured algorithm in primary care screening to avoid missed or delayed diagnosis of dementia. Providing support for the primary care team in the early identification and diagnosis of dementia rather than placing blame is important. A primary care practice is not focused on one stage of the lifespan, or a group of diagnoses and treatments, but a variety of clients along the lifespan and health continuum. 
Delivering provider education, support, staff training, and a point of contact is essential in meeting the needs of the CG/PWD in a family centered care model.

\section{Implications of Qualitative Data.}

The focused qualitative review of participant comments yielded results consistent with the literature (Appendix R). Seven CGs indicated the PWD was either not qualified or not eligible for services (Medicaid or Medicare), or had too much income to receive aid. Paying for out of pocket expenses may be impractical for many CG/PWD dyads. If the PWD has no supplemental service, or even a sliding scale coverage, obtaining support services for the CG/PWD would not be possible. Five respondents indicated either the PWD or the CG did not want a third party in the home ("no stranger in the home"). The literature did not specifically support "no stranger in the home", but CG/PWD dyads did report a desire for continuity of staff and building trusting relationships with staff providing home services (Górska et al., 2013). Macleod, Tatangelo, McCabe, and You (2017) and O'Connell et al. (2012) reported CGs may not use services because of the undesirability of an unknown person in the home, or a lack of stable staff. A possibility is that the respondents in this study were experiencing a slice of Appalachian culture wherein there is a distrust of "outsiders" (Russ, 2010).

The other themes identified by at least four respondents were “don't need additional services/don't need services yet”, “opposition/struggle between CG/PWD about need/refusal of services (Hard for PWD to accept help or PWD may deny need for help), and "wanting permission to seek more resources/support help". For those not needing additional or current services, their needs are met by current services, or the PWD is in an early stage of dementia. One other possibility is the CG does not recognize unmet needs or is uncomfortable finding he/she is not personally meeting all the needs. The CG/PWD opposition/struggle about 
need/refusal of services may be related to the behavior and cognitive process changes of the PWD, or it could be a long-established pattern of the CG/PWD prior relationship. The PWD may have misunderstandings or misconceptions about using a service or receiving support. A PWD may even have paranoia that someone is trying to cause harm or steal something. The CG can be supported and educated on communication techniques with a PWD to help alleviate misunderstandings, clarify expectations, and frame the support service as relevant to the gifts of the PWD. The CGs wanting permission to seek more services or support may be experiencing feelings of being overwhelmed and guilt about being overwhelmed at the same time. The CG needs to have someone recognize he/she is doing a wonderful job in the caregiver role but needs help to keep the role at the high standard. Another possibility is that the CG is resistant to letting anyone else assume any responsibility for the PWD. The CG may not be prepared emotionally to share any part of caregiving with someone else for fear of a negative outcome or what other persons might think if they do accept help.

\section{Health Literacy.}

There is a delicate balance between providing too much information (overwhelming) and too little information about dementia. Health literacy, as defined by the Institute of Medicine (IOM) is "the degree to which individuals have the capacity to obtain, process, and understand basic health information and services needed to make appropriate health decisions" (Ratzan \& Parker, 2000, p. 32). Another warning is providing information to a CG/PWD dyad that is clear and understandable. The CG/PWD dyad must know what dementia is as a disease and how the disease affects the CG and the PWD, that is the way roles change, how family, socialization, finances, and every aspect of life change over an unpredictable period. 


\section{Predisposing Social Support.}

The current study measured social support using CG level of education, CG relationship to PWD, and the Social Provisions Scale Revised. The SPS-10 (Cutrona \& Russell, 1987) measures how CG social connections offer aspects of support, including availability, emotional help, social integration, and encouragement that the CG is valuable as a person. Social support was the strongest predisposing variable, but the weakest to explain variance in non-use of services in this study. The finding is logical, because if a CG has an active social support system, one may expect family or friends to intervene to assist $\mathrm{CG}$ in caregiving role, therefore, the $\mathrm{CG}$ would require fewer services. Tremont et al., (2017) described non-users of services as being older, having more depressive symptoms, and receiving less social support than CGs who were users of services. However, Dam, Vugt, Klinkenberg, Verhay, and Boxtel (2016) reported research studies about social support as inconsistent (some reported positive findings, others no improvement in symptoms), but if a social support intervention worked, multicomponent interventions were the most effective for caregivers. Dam et al. (2016) recognized social support helps decrease CG burden but does not seem to be a mediating factor to CG wellbeing. Social support may be enhanced by attending a dementia caregiver support group. Further investigation of the definition of social support and social networks in relation to CG/PWD is needed to clarify meaning and identify proper measures.

CGs have both emotional and instrumental care needs. Emotional needs can be met by social support, and friends or family who have long term relationships with emotional investments. Home health services or long-term care can meet the instrumental needs of the PWD/CG dyad (bathing, eating, etc.), but the need for emotional care remains. The CG role 
changes throughout the stages of dementia, but the CG role is primary and central to the care of the PWD.

\section{Limitations}

\section{Recruitment and Sample.}

The literature described caregivers as having many time constraints, an increased workload, elevated physical and emotional demands, and the potential for higher stress and depression. Recruitment of these individuals was problematic because of the time factor to complete the survey, the lack of emotional resources the caregiver had to attend to the questionnaire, and the ability of the researcher to contact interested caregivers of persons with dementia.

The sample was recruited from a population of persons identified as having decreased access to care, and there were challenges reaching the dyads of interest. Generalizability of the findings of the study were limited to $\mathrm{CG} / \mathrm{CR}$ in Appalachia. Other disadvantages included the time of 30-45 minutes to complete a questionnaire, as most respondents were busy, and many potential volunteers chose not to complete a research packet.

\section{Surveys.}

There were inherent weaknesses with self-report surveys. The distributed self-report survey had advantages and disadvantages. The advantages included the convenience of collecting substantial amounts of information in a standardized format from many people in an abbreviated period (Kelley, Clark, Brown, \& Sitzia, 2003). Other benefits were the relative low cost, shorter time investment, and ease of statistical analysis of data via software (Kelley et al., 2003; Polit \& Beck, 2014). Self-administered questionnaires offered privacy to respondents, 
eliminated interviewer bias, and allowed participation at a convenient time. One final value, the researcher hoped to obtain many responses, because researchers obtain statistically significant data from a highly representative sample (Kelley et al., 2003). The relative expense $(\$ 2,000)$ of mailing and printing surveys, flyers, and contacting health care providers, area agencies on aging, the Alzheimer's Associations, and other organizations mentioned was not cost prohibitive. Strengths of questionnaires (surveys) were the number of people the researcher accessed, the relative ease of mailing or distributing a hardcopy survey to an eligible participant, comparative reduced cost of mailed surveys versus in-person interviews, and convenience for the participant. Limitations of mailed surveys included sampling error, non-response error, the lack of a resource person to clarify respondent questions about a survey question (although this was offered at two sites, and a phone number and email address of the student researcher were provided, but none were accessed by any respondent), and verification of person completing the questionnaire. Each questionnaire had an assigned study number for each CG/CR dyad, in place of names which helped de-identify the data, and the coded data were stored in a locked file separately from the locked file of consent forms with names of participants. Consent forms included how the data were stored, and information that the data were destroyed five years from the closing date of the study. Additionally, data were analyzed at a group level, rather than individually to further protect individual information. Confidentiality of data and participants from the research team was assured, and research team members were certified via CITI. To examine representativeness of sample, the socio-demographic and CG variables for the population under study (Appalachian counties identified by the Appalachian Regional Commission) were compared to salient data from the United States Census or the National Agencies on Aging. 
Mailed surveys may have been inappropriate for respondents with low literacy rates, or for someone who was forgetful or not answering the questions in a thoughtful manner. Mailed surveys had a low response rate, sometimes around 20\% return (Kelley et al., 2003). Researchers suggested disadvantages of questionnaires also involved lack of validity, because there was not a measure of veracity or intention of the respondent (Polit \& Beck, 2014). Another contention with surveys were the inflexible design of the answer format, in that the answer choices were limited, and may not have been a choice of the respondent (Kelley et al., 2003). A final criticism of distributed surveys was misinterpretation of the question by the respondent, as there was not a researcher available to clarify misconceptions or to address a questions subjectivity with a respondent (Kelley et al., 2003) all the time. The researcher was available at certain times to offer telephone or email questions about the survey, and at times in person where a secluded area was available to allow for maintenance of confidentiality.

\section{Contribution to the Discipline of Nursing.}

The study is guided by Andersen's Behavioral Model of Health Service Use, which is a health systems theory. The study informs the discipline of nursing within this health systems framework . The discipline of nursing according to Newman, Smith, Dexheimer Pharris, and Jones (2008) is the "process as caring in the human health experience" (p. 1). According to Newman et al. (2008), the fundamental concepts are health, caring, consciousness, mutual process, patterning, presence, and meaning. Like Nightingale (2003), health and wholeness of the human being and the essence of nursing practice is a nurse-patient relationship as central to the discipline of nursing (Newman et al., 2008; Nightingale, 2003; Watson, 2008b). Watson (2008a; 2008b) suggested the discipline of nursing is based in Caring Science. She defines Caring Science as "an evolving philosophical-ethical-epistemic field of study, grounded in the 
discipline of nursing and informed by related fields" (Watson, 2008a, p. 18). The discipline guides and informs the practice of nursing (Watson, 2008a).

In the current study, the caring experience is a multi-dimensional process that is ongoing and changing. The CG/PWD dyad experiences a constant accommodation to changing symptoms and suspicion of cognitive changes, then diagnosis and acceptance, along with changing of roles and abilities, that may lead to institutional care. The predisposing, enabling, and need characteristics of Andersen's model clearly contributed to the non-use of services. Caregivers may fear they are being judged as "not good enough" if services, especially formal services, are used. Nursing assistance in helping CG perceive social support and developing services that can be available could decrease service non-use . Caregivers experience loneliness, grief, and have both emotional and instrumental (physical) care demands and changes during the caring process. The findings of this study support the importance of healing relationships (as with their clinicians/nursses) that can support use of services and enhance well-being of CGs and PWDs physical and psychological health. It may be that combining Andersen's model with a more delineated "caring" or nursing model could explain more of the estimate of variance in non-use of services. The challenge of accepting a diagnosis and using services is not unique to the Appalachian culture, and these findings contribute to nursing and the caring experience need.

\section{Recommendations}

Findings from this study suggest more caregiving support resources are necessary and are used more if the physician recommends the assistance. Future studies about CG/PWD non-use of services using Andersen's Behavioral Model of Service Use need to be more uniform in defining and measuring predisposing, enabling, and need factors to provide a clearer understanding of the variables, and to inform policy and practice. Adding a measure of burden might connect 
anecdotal findings from this study to understanding non-use of services. Unexplained variance could also be addressed by qualitative studies about CG/PWD non-use of service to find relevant or complementary theoretical models and offer guidance about combining theoretical models with Andersen's Behavioral Model of Service Use.

\section{Lessons Learned.}

To obtain a larger sample, establishing relationships will be crucial. In their study about Appalachian diabetics, Carpenter and Theeke (2018) recommended using strategies such as careful selection of collection sites (clinics), establishing cooperative relationships with the directors of the research sites, and creating relationships between the research and clinical staff. They used the Purnell Model Concepts which emphasize that communication is essential between the researchers and clinical staff (Carpenter \& Theeke, 2018). The Purnell Model requires sustaining collaborative relationships with research sites and establishing a relationship early in the planning stages of the study. Carpenter \& Theeke used the strategies of "explaining the purpose of the research, offering incentives, and minimizing participant burden” (p. 234). Another important recruitment strategy was the use of cultural competence (Carpenter \& Theeke, 2018). In future studies, using the Purnell Model (Carpenter and Theeke) could improve recruitment of participants. Additionally, future studies should examine the way we teach health care providers about the need for and importance of referrals to identify what is missing and what is essential. Based on the qualitative findings of this study, future research could benefit from including measures of loneliness, CG and PWD fears (such as strangers in the home, financial or physical abuse, or being judged as a failure in the caregiving role) that inhibit the use of support services. 
As recruitment of participants for the study was problematic, further study to determine the best methods of CG of PWD recruitment in Appalachia are needed. Larger samples would support analyses by type of CG and available services in the Appalachian region; future research should show all caregiving resources in the geographical context of the study. Knowledge of what is available may inform survey questions specific to the CG area and if each service is known and used or not. Findings from future studies are needed to determine what services are needed, used, and most effective, allowing policy makers to direct resources to the most needed and effective services. 


\section{References}

Ajzen, I. 1991. The theory of planned behavior. Organizational Behavior and Human Decision Processes, 50: 179-211. doi: 10.1016\%25252F0749-5978\%25252891\%2525

Alligood, M. R., \& Tomey, A. M. (2010). Nursing theorists and their work (7th ed.). Maryland Heights, Missouri: Mosby.

Alzheimer's Association. (2014). Alzheimer's disease facts and figures. Retrieved April, 20, 2014, from http://www.alz.org/alzheimers_disease_facts_and figures.asp\#prevalence

Alzheimer's Association. (2015a). Alzheimer's disease facts and figures. Retrieved June 13, 2015, from http:/www.alz.org/facts/

Alzheimer's Association. (2015b). Legal plans. Retrieved from https://www.alz.org/national/documents/brochure legalplans.pdf

Alzheimer's Association. (2015c). The changing trajectory of Alzheimer's disease: How a treatment by 2025 saves lives and dollars. Retrieved June 13, 2015 from http://www.alz.org/documents_custom/trajectory.pdf

Alzheimer's Association. (2015d). Types of dementia. Retrieved from http://www.alz.org/dementia/types-of-dementia.asp

Alzheimer's Association (2015e). Seven stages of Alzheimer's. Retrieved from http://www.alz.org/nyc/in my community 63259.asp

Alzheimer's Association. (2016). Alzheimer's and Dementia. Retrieved from https://www.alz.org/alzheimer_s_dementia

Alzheimer's Association (2018). Facts and figures. Retrieved from https://www.alz.org/alzheimers-dementia/facts-figures 
Alzheimer's Association Resource Locator. (2018). Community resources. Retrieved from https://www.communityresourcefinder.org/

Alzheimer's Disease International. (2015). About dementia. Retrieved from https://www.alz.co.uk/about-dementia

Alzheimer's Association and National Alliance for Family Caregivers. (2004). Families care: Alzheimers caregiving in the United States. Retrieved from https://www.alz.org/national/documents/report_familiescare.pdf

American Psychological Association. (2006). The high costs of caregiving, from http://www.psychologymatters.org/caregiving.html

American Psychological Association. (2015). About the REACH I study. Retrieved from http://www.apa.org/pi/about/publications/caregivers/practicesettings/intervention/reach.aspx

Andersen, R. (1995). Revisiting the behavioral model and access to medical care: Does it really matter? Journal of Health and Social Behavior, 36, 1-10. Retrieved from http://mph.ufl.edu/files/2012/01/session6april2RevisitingBehavioralModel.pdf

Andersen, R. M. (2008). National health surveys and the Behavioral Model of Health Services Use. Medical Care, 46(7), 647-653. doi: 10.1097/MLR.0b013e31817a835d

Andersen, R., \& Newman, J. F. (1973). Societal and individual determinants of medical care utilization in the United States. Milbank Quarterly, 83(4), Online Only. doi: 10.1111/j.1468-0009.2005.00428.x

Appalachian Regional Commission (ARC). (2018). Counties in Appalachia. Retrieved from https://www.arc.gov/appalachian region/CountiesinAppalachia.asp 
Babitsch, B., Gohl, D., \& von Lengerke, T. (2012). Re-revisiting Andersen’s Behavioral Model of Health Services Use: A systematic review of studies from 1998-2011. GMS PsychoSocial-Medicine, 9. Retrieved from http://www.ncbi.nlm.nih.gov/pmc/articles/PMC3488807/pdf/PSM-09-11.pdf

Bass, D. M., Clark, P. A., Looman, W. J., McCarthy, C. A., \& Eckert, S. (2003). The Cleveland Alzheimer's managed care demonstration: Outcomes after 12 months of implementation. The Gerontologist, 43(1), 73-85 doi: 10.1093/geront/43.1.73

Bass, D. M., Judge, K. S., Snow, A. L., Wilson, N. L., Looman, W. J., McCarthy, C., . . Kunik, M. E. (2012). Negative caregiving effects among caregivers of veterans with dementia. American Journal of Geriatric Psychiatry, 20(3), 239-247. doi:

10.1097/JGP.0b013e31824108ca

Bass, D. M., Judge, K. S., Snow, A. L., ... Kunik, M. E. (2013). Caregiver outcomes of partners in dementia care: Effect of a care coordination program for veterans with dementia and their family members and friends. Journal American Geriatrics Society, 61, 1377-1386. doi: $10.1111 /$ jgs. 12362

Bass, D. M., Judge, K. S., Snow, A. L., Wilson, N. L., Morgan, R. O., Maslow, K., .. Kunik, M. E. (2014). A controlled trial of Partners in Dementia Care: Veteran outcomes after six and twelve months. Alzheimer's Research \& Therapy, 6(1), 9-9. doi:10.1186/alzrt242

Bédard, M., Molloy, D. W., Squire, L., Dubois, S., Lever, J. A., \& O'Donnell, M. (2001). The Zarit burden interview: A new short version and screening version. The Gerontologist, 41(5), 652-657. doi:10.1093/geront/41.5.652

Bekhet, A. K., \& Zauszniewski, J. A. (2008). Theoretical substruction illustrated by the theory of learned resourcefulness. Research and Theory for Nursing Practice, 22(3), 205. 
Belle, S. H., Burgio, L., Burns, R., Coon, D., Czaja, S. J., Gallagher-Thompson, D., . . Song, Z. (2006). Enhancing the quality of life of dementia caregivers from different ethnic or racial groups. Annals of Internal Medicine, 145(10), 727-W216.

Black, B. S., Johnston, D., Morrison, A., Rabins, P. V., Lyketsos, C. G., \& Samus, Q. M. (2012). Quality of life of community-residing persons with dementia based on self-rated and caregiver-rated measures. Quality of Life Research: An International Journal of Quality of Life Aspects of Treatment, Care and Rehabilitation, 21(8), 1379-1389. Retrieved from http://doi.org/10.1007/s11136-011-0044-z

Black, B. S., Johnston, D., Rabins, P. V., Morrison, A., Lyketsos, C., \& Samus, Q. M., (2013). Unmet needs of community residing persons with dementia and their informal caregivers: findings from the maximizing independence at home study. Journal of the American Geriatrics Society, 61(12), 2087-2095. doi: 10.1111/jgs.12549

Brandão, D., Ribeiro, O., \& Martín, I. (2016). Underuse and unawareness of residential respite care services in dementia caregiving: Constraining the Need for Relief. Health \& Social Work, 41(4), 254-262. doi:10.1093/hsw/hlw041

Brodaty, H., Thomson, C., Thompson, C., \& Fine, M. (2005). Why caregivers of people with dementia and memory loss don't use services. International Journal of Geriatric Psychiatry, 20(6), 537-546. doi: 10.1002/gps.1322

Brodaty, H., \& Donkin, M. (2009). Family caregivers of people with dementia. Dialogues in Clinical Neuroscience, 11(2), 217-228. Retrieved from https://www.ncbi.nlm.nih.gov/pmc/articles/PMC3181916/ 
Brown, E. L., Friedemann, M. L., \& Mauro, A. C. (2014). Use of adult day care service centers in an ethnically diverse sample of older adults. Journal of applied gerontology: the official journal of the Southern Gerontological Society, 33(2), 189-206.

Brown, J., \& Chen, S. (2008). Help-seeking patterns of older spousal caregivers of older adults with dementia. Issues in Mental Health Nursing, 29(8), 839-852. doi: $10.1080 / 01612840802182854$

Brown, J. W., Chen, S.-1., Mitchell, C. \& Province, A. (2007). Help-seeking by older husbands caring for wives with dementia. Journal of Advanced Nursing, 59(4), 352-360. doi: $10.1111 /$ j.1365-2648.2007.04290.x

Bynum, J. W. (2014). The long reach of Alzheimer's disease: Patients, practice, and policy. Health Affairs, 33(4), 534-540. doi:10.1377/hlthaff.2013.1247

Carpenter, R., \& Theeke, L. A. (2018). Strategies for recruiting a sample of adults with type 2 diabetes from primary care clinics in rural Appalachia: Incorporating cultural competence. International Journal of Nursing Sciences, 5(3), 230-237. doi:https://doi.org/10.1016/j.ijnss.2018.06.003

Coon, D.W., Besst, D.A., Doucet, J.S., Chavez, A., Fenzi, M., Raach, K., Schaus, D., Vargo, C., Harmon, J.R., Pratt, A., Hawthornthwaite, S., \& Hirsch, S. (2016). CarePRO: Embedding an evidence-based intervention for caregiver empowerment. Arizona Geriatrics Society, 22, 9-11. Retrieved from http://www.arizonageriatrics. org/assets/Journals/2016\%20azgs\%20fall\%20journal\%20lowresolution\%20final\%20proof\%20-\%2010\%205\%2016.pdf

Cordell, C. B., Borson, S., Boustani, M., Chodosh, J., Reuben, D., Verghese, J., . . Fried, L. B. (2013). Alzheimer's Association recommendations for operationalizing the detection of 
cognitive impairment during the Medicare Annual Wellness Visit in a primary care setting. Alzheimer's \& Dementia, 9(2), 141-150.

doi:https://doi.org/10.1016/j.jalz.2012.09.011

Cox, C. B., \& Pardasani, M. (2013). Alzheimer's in the workplace: a challenge for social work. Journal Of Gerontological Social Work, 56(8), 643-656. doi:10.1080/01634372.2013.821693

Cunningham, C., \& Hennessy, E. (2015). Co-morbidity and systemic inflammation as drivers of cognitive decline: New experimental models adopting a broader paradigm in dementia research. Alzheimer's Research and Therapy, 7(33). 1-13. doi:10.1186/s13195-015-01172

Cuoto, R. A. (2006). Appalachia. In P. J. Obermiller \& M. E. Maloney (Eds.), Appalachia: Social context past and present (5th ed., pp. 3-14). Dubuque, IA: Kendall/Hunt Publishing Company.

Cutrona, C. E., \& Russell, D. W. (1987). The provisions of social relationships and adaptation to stress. Advances in Personal Relationships, 1, 37-67. Retrieved from http://depts.washington.edu/uwcssc/sites/default/files//hw00/d40/uwcssc/sites/default/file s/Social $\% 20$ Provisions $\% 20$ Scale $0 . p d f$

Dam, A. E. H., de Vugt, M. E., Klinkenberg, I. P. M., Verhey, F. R. J., \& van Boxtel, M. P. J. (2016). A systematic review of social support interventions for caregivers of people with dementia: Are they doing what they promise? Maturitas, 85, 117-130. Retrieved from http://www.sciencedirect.com/science/article/pii/S037851221530092X. doi:https://doi.org/10.1016/j.maturitas.2015.12.008 
Daulatzai, M. (2013). Neurotoxic saboteurs: Straws that break the hippo's (Hippocampus) back drive cognitive impairment and Alzheimer's disease. Neurotoxicity Research, 24(3), 407459. doi:10.1007/s12640-013-9407-2

Dizazzo-Miller, R., Pociask, F., \& Samuel, P. (2013). Understanding resource needs of persons with dementia and their caregivers. Michigan Family Review, 17(1), 1-20. Retrieved from https://quod.lib.umich.edu/m/mfr/4919087.0017.102/--understanding-resource-needs-ofpersons-with-dementia?rgn=main. doi:http://dx.doi.org/10.3998/mfr.4919087.0017.102

Dukes Holland, K., \& Holahan, C. (2003). The relation of social support and coping to positive adaptation to breast cancer. Psychology and Health, 18(1), 15-29.

doi:10.1080/0887044031000080656

Eichler, T., Thyrian, J. R., Hertel, J., Michalowsky, B., Wucherer, D., Dreier, A., ... Hoffmann, W. (2015). Rates of formal diagnosis of dementia in primary care: The effect of screening. Alzheimer's \& Dementia: Diagnosis, Assessment \& Disease Monitoring, 1(1), 87-93. Retrieved from http://www.sciencedirect.com/science/article/pii/S2352872915000111. doi:https://doi.org/10.1016/j.dadm.2014.11.007

Eichler, T. S., Thyrian, J. R., \& Hoffmann, W. (2014). Rates of formal diagnosis in people screened positive for dementia in primary care and the effect of screening on diagnosis rates: Results of the DelpHhi trial. Alzheimer's \& Dementia: The Journal of the Alzheimer's Association, 10(4), P610-P611. Retrieved from https://doi.org/10.1016/j.jalz.2014.05.1038. doi:10.1016/j.jalz.2014.05.1038

Ervin, K., \& Reid, C. (2015). Service utilisation by carers of people with dementia in rural Victoria. Australasian Journal on Ageing, 34(4), E1-E6. doi:10.1111/ajag.12162 
Family Caregiver Alliance National Center on Caregiving (FCANCC). (2014, December 13, 2014). Caregivers count too! Section 3: What should family caregiver assessments include? Retrieved December 15, 2014, 2014, from https://caregiver.org/caregiverscount-too-s3-what-should-assessments-include

Family Caregiver Alliance: National Center on Caregiving (FCANCC) and the Benjamin Rose Institute on Aging: The Margaret Blenkner Research Institute. (2012). Selected caregiver assessment measures: A resource inventory for practitioners $2^{\text {nd }}$ edition (Family Caregiver Alliance (FCA) and the Benjamin Rose Institute on Aging, Trans.) Selected caregiver assessment measures: A resource inventory for practitioners (2nd ed., pp. 79): Family Caregiver Alliance (FCA) and the Benjamin Rose Institute on Aging

Faul, F., Erdfelder, E., Buchner, A., \& Lang, A. (2009). Statistical power analyses using G*Power 3.1: Tests for correlation and regression analyses. Behavior Research Methods, 41(4), 1149-1160. doi:10.3758/brm.41.4.1149

Faul, F., Erdfelder, E., Lang, A.-G., \& Buchner, A. (2007). G*Power 3: A flexible statistical power analysis program for the social, behavioral, and biomedical sciences. Behavior Research Methods, 39(2), 175-191.

Fishbein, M. and Ajzen, I. 1975. Belief, attitudes, intention and behaviour: an introduction to theory and research, Reading, MA: Addison-Wesley.

Flynn, R., \& Mulcahy, H. (2013). Early-onset dementia: The impact on family care-givers. British Journal Of Community Nursing, 18(12), 598-606. Retrieved from: http://search.ebscohost.com/login.aspx?direct=true\&db=hch\&AN=93253470\&site=ehost $\underline{\text {-live }}$ 
Foldes, S. S., Moriarty, J. P., Farseth, P. H., Mittelman, M. S., \& Hall Long, K. (2018). Medicaid Savings From The New York University Caregiver Intervention for Families with Dementia. Gerontologist, 58(2), e97-e106. doi:10.1093/geront/gnx077

Forbes, D., Strain, L., Blake, C., Peacock, S., Harrison, W., Woytkiw, T., .. . Gibson, M. (2015). Dementia care evidence: Contextual dimensions that influence use in Canadian northern rural home care centres. Online Journal of Rural Nursing \& Health Care, 15(1), 117-149. Retrieved from http://search.ebscohost.com/login.aspx?direct=true\&db=c8h\&AN=103297498\&site=ehos t-live. doi:10.14574/ojrnhc.v15i1.344

Fraenkel, R. J., \& Wallen, E. N. (2000). How to design and evaluate research in education (4th ed.). San Francisco: McGraw-Hill.

Funkhouser, E., Vellala, K., Baltuck, C., Cacciato, R., Durand, E., McEdward, D., . . National Dental, P. C. G. (2017). Survey methods to optimize response rate in the national dental practice-based research network. Evaluation \& the Health Professions, 40(3), 332-358. doi:10.1177/0163278715625738

Garre-Olmo, J., López-Pousa, S., Vilalta-Franch, J., de Gracia, B. M., \& Vilarrasa, A. B. (2009). Grouping and trajectories of the neuropsychiatric symptoms in patients with Alzheimer's disease, part I: Symptom clusters. Journal of Alzheimer's disease: JAD, 22(4), 11571167. doi: 10.3233/JAD2010101212

Gaugler, J. E., Mittelman, M. S., Hepburn, K., \& Newcomer, R. (2010). Clinically significant changes in burden and depression among dementia caregivers following nursing home admission. BMC Medicine, 8, 85-85. doi:10.1186/1741-7015-8-85 
Gaugler, J. E., Roth, D. L., Haley, W. E., \& Mittelman, M. S. (2008). Can counseling and support reduce burden and depressive symptoms in caregivers of people with Alzheimer's Disease during the transition to institutionalization? Results from the New York University Caregiver Intervention Study. Journal of the American Geriatrics Society, 56(3), 421-428. doi:10.1111/j.1532-5415.2007.01593.x

Gauthier, S., Reisberg, B., Zaudig, M., Petersen, R. C., Ritchie, K., Broich, K., ... Winblad, B. (2006). Mild cognitive impairment. Lancet, 367(9518), 1262-1270. doi:10.1016/S0140$6736(06) 68542-5$

Gerdner, L. A., Tripp-Reimer, T., \& Simpson, H. C. (2007). Hard lives, God's help, and struggling through: Caregiving in Arkansas delta. Journal of Cross Cultural Gerontology, 22, 355-374. doi: 10.1007/s10823-007-9047-1

Gitlin, L. N., \& Hodgson, N. A. (2016). Who should assess the needs of and care for a dementia patient's caregiver? AMA Journal of Ethics, 18(12), 1171-1181. Retrieved from https://journalofethics.ama-assn.org/article/who-should-assess-needs-and-care-dementiapatients-caregiver/2016-12. doi:10.1001/journalofethics.2016.18.12.ecas1-1612.

Gitlin, L. N., Burgio, L. D., Mahoney, D., Burns, R., Zhang, S., Schulz, R., . . Ory, M. G. (2003). Effect of multicomponent interventions on caregiver burden and depression: The Resources for Enhancing Alzheimer's Caregiver Health (REACH) multisite initiative at 6-month follow-up. Psychology and Aging, 18(3), 361-374. doi:10.1037/0882-7974.18.3.361

Gitlin, L. N., Roth, D. L., \& Huang, J. (2014). Mediators of the impact of a home-based intervention (beat the blues) on depressive symptoms among older African Americans. 
Psychology and aging, 29(3), 601-611. Retrieved from https://www.ncbi.nlm.nih.gov/pmc/articles/PMC4176772/ doi:10.1037/a0036784

Gitlin, L. N., Winter, L., Dennis, M. P., Hodgson, N., Hauck, W. W., Gitlin, L. N., . . \& Hauck, Walter. W. (2010). A biobehavioral home-based intervention and the well-being of patients with dementia and their caregivers: The COPE randomized trial. JAMA: Journal of the American Medical Association, 304(9), 983-991. doi:10.1001/jama.2010.1253

Goins, R. T., Spencer, S. M., \& Byrd, J. C. (2009). Research on rural caregiving. Journal of Applied Gerontology, 28(2), 139-170. doi: 10.1177/0733464808326294

Górska, S., Forsyth, K., Irvine, L., Maciver, D., Prior, S., Whitehead, J., . . \& \& Reid, J. (2013). Service-related needs of older people with dementia: perspectives of service users and their unpaid carers. International Psychogeriatrics, 25(7), 1107-1114. doi:10.1017/S1041610213000343

Grober, E., Wakefield, D., Ehrlich, A. R., Mabie, P., \& Lipton, R. B. (2017). Identifying memory impairment and early dementia in primary care. Alzheimer's \& Dementia: Diagnosis, Assessment \& Disease Monitoring, 6, 188-195. Retrieved from http://www.sciencedirect.com/science/article/pii/S2352872917300064. doi:https://doi.org/10.1016/j.dadm.2017.01.006

Hall, G. R., \& Buckwalter, K. C. (1987). Progressively lowered stress threshold: A conceptual model for care of adults with Alzheimer's disease. Archives of Psychiatric Nursing, 1, $399-406$.

Hamuro, A., Isono, H., Sugai, Y., Torii, S., Furuta, N., Mimura, M., \& Kamijima, K. (2007). Behavioral and psychological symptoms of dementia in untreated Alzheimer's disease patients. Psychogeriatrics, 7(1), 4-7. doi:10.1111/j.1479-8301.2006.00153.x 
Harrison, F., Low, L. F., Barnett, A., Gresham, M., \& Brodaty, H. (2014). What do clients expect of community care and what are their needs? The community care for the elderly: Needs and Service Use Study (CENSUS). Australasian journal on ageing, 33(3), 206-213. doi:10.1111/ajag.12118

Harrow, B. S., Mahoney, D. F., Mendelsohn, A. B., Ory, M. G., Coon, D. W., Belle, S. H., \& Nichols, L. O. (2004). Variation in cost of informal caregiving and formal-service use for people with Alzheimer's disease. American Journal of Alzheimer's Disease \& Other Dementias $^{\circledR}, 19(5), 299-308$. doi:10.1177/153331750401900507

Heinrich, S., Laporte Uribe, F., Wübbeler, M., Hoffmann, W., \& Roes, M. (2016). Knowledge evaluation in dementia care networks: a mixed-methods analysis of knowledge evaluation strategies and the success of informing family caregivers about dementia support services. International Journal of Mental Health Systems, 10, 1-10. doi:10.1186/s13033016-0100-8

Hoven, M. R. (2012). Investigating the relationships between perceived social support and parent self-efficacy in parents of preschool-aged children: (Master of Arts Thesis). University of British Columbia. Retrieved from https://open.library.ubc.ca/cIRcle/collections/24/items/1.0073240

Howe, A. L., Schofield, H., \& Herrman, H. (1997). Caregiving: A common or uncommon experience? Social Science \& Medicine, 45(7), 1017-1029. doi:10.1016/S02779536(97)00017-8

Ingebrestsen, R., \& Solem, P. E. (1998). Spouses of persons with dementia: Attachment, loss and coping. [Journal]. Norwegian Journal of Epidemiology, 8(2), 149-156. Retrieved from 
file://C:/Users/Kristi\%20Childers/Downloads/457-Article\%20Text-1729-1-1020091029.pdf

Iapichino, E., Rucci, P., Corbani, I. E., Apter, G., Quartieri Bollani, M., Cauli, G. , Gala, C., and Bassi, M. (2016). Development and validation of an abridged version of the Social Provisions Scale (SPS-10) in Italian. Journal of Psychopathology, 22. 157-163. Retrieved from http://www.jpsychopathol.it/wp-content/uploads/2016/05/08_Art_Ass_Rucci1.pdf

Jarrott, S. E., Zarit, S. H., Stephens, M. A. P., Townsend, A., \& Greene, R. (2005). Instrumental help and caregivers' distress: Effects of change in informal and formal help. American Journal of Alzheimer's Disease \& Other Dementias ${ }^{\circledR}, 20(3), 181-190$. doi:10.1177/153331750502000308

Johnston, D., Samus, Q., Morrison, A., Leoutsakos, J., Hicks, K., Handel, S., ... Black, B. (2011). Identification of community-residing individuals with dementia and their unmet needs for care. International Journal of Geriatric Psychiatry, 26(3), 292-298. http://doi.org/10.1002/gps.2527

Kelley, K., Clark, B., Brown, V., \& Sitzia, J. (2003). Good practice in the conduct and reporting of survey research. International Journal for Quality in HealthCare, 15(3), 261-266. Retrieved from http://intqhc.oxfordjournals.org/content/intqhc/15/3/261.full.pdf

Kosberg, J. I., Kaufman, A. V., Burgio, L. D., Leeper, J. D., \& Sun, F. (2007). Family caregiving to those with dementia in rural Alabama. [Article]. Journal of Aging \& Health, 19(1), 321. doi: $10.1177 / 0898264306293604$

Krause, N., \& Markides, K. (1990). Measuring social support among older adults. The International Journal of Aging and Human Development, 30(1), 37-53. doi: https://doi.org/10.2190/CY26-XCKW-WY1V-VGK3 
Kuhn, D., Hollinger-Smith, L., Presser, J., Civian, J., \& Batsch, N. (2008). Powerful tools for caregivers online: An innovative approach to support employees. Journal of Workplace Behavioral Health, 23(1/2), 51-69.

Lawton, M. P., Kleban, M. H., Moss, M., Rovine, M., \& Glicksman, A. (1989). Measuring caregiving appraisal. Journal of Gerontology: Psychological Sciences, 44(3), 61-71. doi: 10.1093/geronj/44.3.P61

Lawton, M. P., Moss, M., Fulcomer, M., \& Kleban, M. (1982). A research and service oriented multi-level assessment instrument. Journal of Gerontology, 37(1), 91-99. doi\#: 10.1093/geronj/37.1.91

Lawton, M. P., Moss, M., Fulcomer, M., \& Kleban, M. (2013). Madlyn and Leonard Abramson Center for Jewish Life (formerly Philadelphia Geriatric Center) multi-level assessment instrument manual for full length MAI (PGC-MAI). Retrieved from https://www.abramsoncenter.org/media/1201/lawtons-pgc-multi-level-assessmentinstrument.pdf

Lawton, M. P., Moss, M., Hoffman, C., \& Perkinson, M. (2000). Two transitions in daughter's caregiving careers. The Gerontologist, 40(4), 431-448. doi: 10.1093/geront/40.4.437

Lincoln, Y. S., \& Guba, E. G. (1985). Naturalistic inquiry. Newbury Park, CA: SAGE Publications.

Liu, Y., Almeida, D. M., Rovine, M. J., \& Zarit, S. H. (2018). modeling cortisol daily rhythms of family caregivers of individuals with dementia: Daily stressors and adult day services Use. Journals of Gerontology Series B: Psychological Sciences \& Social Sciences, 73(3), 457-467. doi:10.1093/geronb/gbw140 
Long, E. (2018). 2018 National Alzheimers and Dementia Resource Center (NADRC): Evaluating dementia services and supports-Instrument resource list. Retrieved from National Alzheimer's and Dementia Resource Center: https://nadrc.acl.gov/node/70

Loos, C., \& Bowd, A. (1997). Caregivers of persons with Alzheimer's disease: some neglected implications of the experience of personal loss and grief. Death Studies, 21(5), 501-514. doi:10.1080/074811897201840

Lykens, K., Moayad, N., Biswas, S., Reyes-Ortiz, C., \& Singh, K. P. (2014). Impact of a community based implementation of REACH II Program for caregivers of Alzheimer's patients. PLoS ONE, 9(2), 1-7. doi:10.1371/journal.pone.0089290

Macleod, A., Tatangelo, G., McCabe, M., \& You, E. (2017). "There isn't an easy way of finding the help that's available." Barriers and facilitators of service use among dementia family caregivers: a qualitative study. International Psychogeriatrics, 29(5), 765-776. doi:10.1017/S1041610216002532

Martindale-Adams, J., Nichols, L. O., Zuber, J., Burns, R., \& Graney, M. J. (2016). Dementia caregivers' use of services for themselves. The Gerontologist, 56(6), 1053-1061. doi:10.1093/geront/gnv121

Mast, M. E. (2013). To use or not to use. A literature review of factors that influence family caregivers' use of support services. Journal Of Gerontological Nursing, 39(1), 20-28. Retrieved from http://search.ebscohost.com/login.aspx?direct=true $\& d b=$ cmedm\&AN=23413449\&site $=e$ host-live.

Masumi, S., Hayat, J., Meguro, K., Thet, O. O., Jafri, S., Atsushi, Y., ... \& Reisberg, B. (2003). Correlation between functional assessment staging and the 'Basic Age' by the Binet scale 
supports the retrogenesis model of Alzheimer's disease: a preliminary study.

Psychogeriatrics, 3(2), 82. doi:10.1046/j.1479-8301.2003.00015.x

Mcgarvey, E. L., Leon-verdin, M., Killos, L. F., Guterbock, T., \& Cohn, W. F. (2011). Health disparities between appalachian and non-appalachian counties in Virginia USA. Journal of Community Health, 36(3), 348-356. doi:10.1007/s10900-010-9315-9

Morgan, D. G., Semchuk, K. M., Stewart, N. J., \& D’Arcy, C. (2002). Rural families caring for a relative with dementia: Barriers to use of formal services. Social Science \& Medicine, 55(7), 1129-1142. doi: http://dx.doi.org/10.1016/S0277-9536(01)00255-6

Morhardt, D. (2011). Accessing community-based and long-term care services: Challenges facing persons with Frontotemporal Dementia and their families. Journal of Molecular Neuroscience, 45(3), 737-741. doi:10.1007/s12031-011-9612-5

Murphy, B., Schofield, H., Nankervis, J., Bloch, S., Herrman, H., \& Singh, B. (1997). Women with multiple roles: The emotional impact of caring for ageing parents. Ageing and Society, 17(May), 277-291.

Murray, S. A., Kendall, M., Boyd, K., \& Sheikh, A. (2005). Illness trajectories and palliative care. BMJ: British Medical Journal, 330(7498), 1007-1011. doi:10.1136/bmj.330.7498.1007

National Association of Area Agencies on Aging (2015). Aging services. Retrieved from http://www.n4a.org/

National Institutes of Health (NIH), National Institute on Aging (NIA) and National Institute of Neurological Disorders and Stroke. (2013). Lewy body dementia: Information for patients, families, and professionals [Pub ID: 13-7907]. Retrieved from 
http://www.ninds.nih.gov/disorders/dementiawithlewybodies/dementiawithlewybodies.ht

$\underline{\mathrm{m}}$

Neville, C., Beattie, E., Fielding, E., \& MacAndrew, M. (2015). Literature review: Use of respite by carers of people with dementia. Health \& Social Care in the Community, 23(1), 51-63. Retrieved from http://search.ebscohost.com/login.aspx?direct=true\&db=c8h\&AN=103921927\&site=ehos t-live. doi:10.1111/hsc. 12095

Newman, M. A., Smith, M. C., Pharris, M. D., \& Jones, D. (2008). The focus of the discipline revisited. Advances in Nursing Science, 31(1), E16-E27.

doi:10.1097/01.ANS.0000311533.65941.f1

Nightingale, F. (2003). Notes on nursing: What it is, and what it is not. New York: Barnes \& Noble Books.

O'Connell, B., Hawkins, M., Ostaszkiewicz, J., \& Millar, L. (2012). Carers' perspectives of respite care in Australia: An evaluative study. Contemporary Nurse: A Journal for the Australian Nursing Profession, 41(1), 111-119. Retrieved from http://search.ebscohost.com/login.aspx?direct=true\&db=c8h\&AN=2011678935\&site=eh $\underline{\text { ost-live }}$

Orpin, P., Stirling, C., Hetherington, S., \& Robinson, A. (2014). Rural dementia carers: Formal and informal sources of support. Ageing and Society, 34(2), 185-208. doi:10.1017/s0144686x12000827

Ory, M. G., Hoffman, R., R., Yee, J., L., Tennstedt, S., \& Schulz, R. (1999). Prevalence and impact of caregiving: A detailed comparison between dementia and nondementia 
caregivers. The Gerontologist, 39(2). 177-185. Retrieved from http://www.caregiving.org/pdf/research/Ory_Schulz_NAC_survey.pdf

Pallant, J. (2012). SPSS survival manual: A step by step guide to data analysis using SPSS for Windows (4th ed.). New York: McGraw-Hill: Open University Press.

Parveen, S., Peltier, C., \& Oyebode, J. R. (2017). Perceptions of dementia and use of services in minority ethnic communities: A scoping exercise. Health \& Social Care in the Community, 25(2), 734-742. doi:10.1111/hsc.12363

Pathak, K. P., \& Montgomery, A. (2015). General practitioners' knowledge, practices, and obstacles in the diagnosis and management of dementia. Aging \& Mental Health, 19(10), 912-920. Retrieved from http://search.ebscohost.com/login.aspx?direct=true\&db=c8h\&AN=109816087\&site=ehos t-live. doi:10.1080/13607863.2014.976170

Pearlin, L. I., Mullan, J. T., Semple, S. J., \& Skaff, M. M. (1990). Caregiving and the stress process: An overview of concepts and their measures. The Gerontologist, 30(5), 583-594. Retrieved from https://doi.org/10.1093/geront/30.5.583

Peterson, K., Hahn, H., Lee, A. J., Madison, C. A., \& Atri, A. (2016). In the Information Age, do dementia caregivers get the information they need? Semi-structured interviews to determine informal caregivers' education needs, barriers, and preferences. $B M C$ Geriatrics, 16, 164. Retrieved from http://www.ncbi.nlm.nih.gov/pmc/articles/PMC5035467/. doi:10.1186/s12877-016-0338- 
Phillipson, L., \& Jones, S. C. (2010). Thematic analysis of focus groups with dementia caregivers: Beliefs that influence the use of respite services. Centre for Health Initiatives, University of Wollongong, Wollongong, NSW, Australia [unpublished manuscript].

Phillipson, L., \& Jones, S. C. (2011). Residential respite care: The caregiver's last resort. Journal of Gerontological Social Work, 54(7), 691-711.

doi:http://dx.doi.org/10.1080/01634372.2011.593613

Phillipson, L., \& Jones, S. C. (2012). Use of day centers for respite by help-seeking caregivers of individuals with dementia. Journal of Gerontological Nursing, 38(4), 24-34. doi: 10.3928/00989134-20120307-05

Phillipson, L., Jones, S. C., \& Magee, C. (2014). A review of the factors associated with the nonuse of respite services by carers of people with dementia: Implications for policy and practice. . Health \& Social Care in the Community, 22(1), 1-12. doi: 10.1111/hsc.12036

Phillipson, L., Magee, C., \& Jones, S. C. (2013). Why carers of people with dementia do not utilise out-of-home respite services. Health \& Social Care in the Community, 21(4), 411422. doi: $10.1111 /$ hsc. 12030

Ploeg, J., Denton, M., Tindale, J., Hutchison, B., Brazil, K., Akhtar-Danesh, N., . . Plenderleith, J. M. (2009). Older adults' awareness of community health and support services for dementia care. Canadian Journal on Aging, 28(4), 359-370. doi: http://dx.doi.org.www.libproxy.wvu.edu/10.1017/S0714980809990195

Polit, D. F., \& Beck, C. T. (2008). Nursing research: Generating and assessing evidence for nursing practice (8th ed.). Philadelphia: Lippincott Williams and Wilkins.

Polit, D. F., \& Beck, C. T. (2014). Essentials of Nursing Research (8th ed.). Philadelphia: Lippincott Williams \& Wilkins. 
Potter, A. J. (2018). Factors associated with caregivers use of support services and caregivers' nonuse of services sought. Journal of Aging, 30(2), 155. doi:10.1080/08959420.2017.1414539

Prichep, L. S., John, E. R., Ferris, S. H., Rausch, L., Fang, Z., ... \& Reisberg, B. (2006 March). Prediction of longitudinal cognitive decline in normal elderly with subjective complaints using electrophysiological imaging, Neurobiology of Aging, 27(3), 471-481, doi: http://dx.doi.org/10.1016/j.neurobiolaging.2005.07.021

Radloff, L. (1977). The CES-D scale: A self-report depression scale for research in the general population. Applied Psychological Measurement, 1(3), 385-401. doi:10.1177/014662167700100306

Rajmohan, V., \& Mohandas, E. (2007). The limbic system. Indian Journal of Psychiatry, 49(2), 132-139. doi:10.4103/0019-5545.33264

Ratzan, S. C., \& Parker, R. M. (2000). Introduction In: National Library of Medicine Current Bibliographies in Medicine: Health Literacy. In C. R. Selden, Zorn, M., Ratzan, S. C., Parker, R. M. (Ed.). Bethesda, MD: National Institutes of Health, U.S. Department of Health and Human Services. Retrieved from https://www.ncbi.nlm.nih.gov/books/NBK216033/?report=reader

Rea, P. (2015). Essential clinical anatomy of the nervous sytem. [e-book]. St. Louis, MO, USA: Aademic Press, ProQuest ebrary via WVU library.

Reed, B. R. (2012). Alzheimer's Disease and caregiving. Retrieved from the Family Caregiver Alliance website https://www.caregiver.org/alzheimers-disease-caregiving

Reinhard, S. C., Given, B., Petlick, N. H., \& Bemis, A. (2008). Family caregiving and caregiver assessment. In R. G. Hughes (Ed.), Patient safety and quality: An evidence-based 
handbook for nurses (pp. 1-64). Rockville, MD: Agency for Healthcare Research and Quality (AHRQ). Retrieved from http://www.ahrq.gov/professionals/cliniciansproviders/resources/nursing/resources/nurseshdbk/index.html.

Reisberg, B., \& Franssen, E. H. (1999). Clinical stages of Alzheimer's disease. An atlas of Alzheimer's disease. New York, London: The Parthenon Publishing Group, 11-29.

Reisberg, B., Monteiro, I., Torossian, C., Auer, S., Shulman, M. B., Ghimire, S., . . Xu, J. (2014). The BEHAVE-AD assessment system: A perspective, a commentary on new findings, and a historical review. Dementia and geriatric cognitive disorders, 38(1-2), 89-146. doi:10.1159/000357839

Reuben, D. B., Evertson, L. C., Wenger, N. S., Serrano, K., Chodosh, J., Ercoli, L., \& Tan, Z. S. (2013). The University of California at Los Angeles Alzheimer's and Dementia Care Program for comprehensive, coordinated, patient-centered care: Preliminary data. Journal of the American Geriatrics Society, 61(12), 2214-2218. doi:10.1111/jgs.12562

Robinson, A., Lea, E., Hemmings, L., Vosper, G., McCann, D., Weeding, F., \& Rumble, R. (2012). Seeking respite: Issues around the use of day respite care for the carers of people with dementia. Ageing \& Society, 32(2), 196-218. doi: 10.1017/S0144686X11000195

Robinson, K. M., Buckwalter, K., \& Reed, D. (2013). Differences between dementia caregivers who are users and nonusers of community services. Public Health Nursing, 30(6), p501510. doi: $10.1111 / \mathrm{phn} .12041$

Robinson, K. M., Buckwalter, K. C., \& Reed, D. (2005). Predictors of the use of services among dementia caregivers. Western Journal of Nursing Research, 27(126-1402). doi: $10.1177 / 0193945904272453$ 
Robinson, L., Clare, L., \& Evans, K. (2005). Making sense of dementia and adjusting to loss: Psychological reactions to a diagnosis of dementia in couples. Aging \& Mental Health, 9(4), 337-347. Retrieved from http://search.ebscohost.com/login.aspx?direct=true\&db=c8h\&AN=2009037716\&site=eh $\underline{\text { ost-live }}$

Rosswurm, M. A., Dent, D. M., Armstrong-Persily, C., Woodburn, P., \& Davis, B. (1996). Illness experiences and health recovery behaviors of patients in southern Appalachia. Western Journal of Nursing Research, 18(4), 441-459.

Russ, K. A. (2010). Working with clients of Appalachian culture. Retrieved from https://www.counseling.org/resources/library/VISTAS/2010-V-Online/Article_69.pdf

Russell, D., Cutrona, C. E., Rose, J., \& Yurko, K. (1984). Social and emotional loneliness: An examination of Weiss's typology of loneliness. Journal of Personality and Social Psychology, 46(6), 1313-1321. doi: http://dx.doi.org/10.1037/0022-3514.46.6.1313

Russell, D. W., \& Cutrona, C. E. (1991). Social support, stress, and depressive symptoms among the elderly: Test of a process model. Psychology and Aging, 6(2), 190-201. doi:10.1037/0882-7974.6.2.190\

Sadler, G. R., Lee, H. C., Lim, R. S. H., \& Fullerton, J. (2010). Research article: Recruitment of hard-to-reach population subgroups via adaptations of the snowball sampling strategy. Nursing \& Health Sciences, 12(3), 369-374. doi:10.1111/j.1442-2018.2010.00541.x

Schofield, H. (1998). Family caregivers: Disability, illness and ageing London: St. Leonards: Allen \& Unwin in association with the Victorian Health Promotion Foundation; [London: UCL] [distributor], 1998. 
Schofield, H. L., Bloch, S., Nankervis, J., Murphy, B., Singh, B.S., \& Herman, H. E. (1999). Health and well-being of women family carers: A comparative study with a generic focus. Australian and New Zealand Journal of Public Health, 23(6), 585-589. Retrieved from http://search.proquest.com/docview/215709096?accountid=2837

Schofield, H., \& Herrman, H. (1993). Characteristics of carers in Victoria. Family Matters, (34), 21-26. Retrieved from Australian Institute of Family Studies website: http://www.aifs.gov.au/institute/pubs/fm1/fm34hs.html

Schofield, H. L., Herrman, H. E., Bloch, S., Howe, A. \& Singh, B. (1997). A profile of Australian family caregivers: Diversity of roles and circumstances. Australian and New Zealand Journal of Public Health, 21(1), 59-66. doi: 10.1111/j.146742X.1997.tb01655.x

Schofield, H., Murphy, B., Bloch, S., Herrman, H., \& Singh, B. (1997). Family caregiving: measurement of emotional wellbeing and various aspects of the caregiving role. Psychological Medicine, 27, 647-57. Retrieved from https://www.ncbi.nlm.nih.gov/pubmed/9153685

Schulz, R. (2006). Resources for Enhancing Caregiver Health (R.E.A.C.H.), 1996-2001, baseline and follow up data. Retrieved from: https://www.icpsr.umich.edu/icpsrweb/ICPSR/studies/03678

Sinclair, M., O’Toole, J., Malawaraarachchi, M., \& Leder, K. (2012). Comparison of response rates and cost-effectiveness for a community-based survey: Postal, internet and telephone modes with generic or personalised recruitment approaches. BMC Medical Research Methodology, 12, 132-132. doi:10.1186/1471-2288-12-132 
Singh, P., Hussain, R., Khan, A., Irwin, L., \& Foskey, R. (2014). Dementia care: Intersecting informal family care and formal care systems. Journal of Aging Research, 2014, 9. Retrieved from http://dx.doi.org/10.1155/2014/486521. doi:10.1155/2014/486521

Standford Medicine Older Adult and Family Center (SMOAFC). (2015). REACH I \& II: Resources for Enhancing Alzheimer's Caregiver Health. Retrieved from http://oafc.stanford.edu/projects/reach.html

Stockwell-Smith, G., Kellett, U., \& Moyle, W. (2010). Why carers of frail older people are not using available respite services: An Australian study. Journal of Clinical Nursing, 19(1314), 2057. doi:10.1111/j.1365-2702.2009.03139.x

Sun, F., Roff, L. L., Klemmack, D., \& Burgio, L. D. (2008). The influences of gender and religiousness on Alzheimer disease caregivers' use of informal support and formal services. Journal Of Aging And Health, 20(8), 937-953. doi: 10.1177/0898264308324652

Takai, Y., Yamamoto-Mitani, N., Okamoto, Y., Fukahori, H., Ko, A., \& Tanaka, M. (2013). Family caregiver strategies to encourage older relatives with dementia to use social services. Journal of Advanced Nursing, 69(12), 2675-2685. Retrieved from http://search.ebscohost.com/login.aspx?direct=true\&db=c8h\&AN=104157617\&site=ehos t-live. doi:10.1111/jan.12155

Thorpe, J. M., Van Houtven, C. H., Sleath, B. L., \& Thorpe, C. T. (2010). Rural-urban differences in preventable hospitalizations among community-dwelling veterans with dementia. The Journal of Rural Health, 26(2), 146-155. doi: 10.1111/j.17480361.2010.00276.x 
Torraco, R. J. (2005). Writing integrative literature reviews: Guidelines and examples. Human Resource Development Review, 4(3), 356-367. Retrieved from http://search.proquest.com/docview/221810269?accountid=2837

Tremont, G., Davis, J. D., Ott, B. R., Galioto, R., Crook, C., Papandonatos, G. D., ... Bishop, D. S. (2017). Randomized trial of the family intervention: Telephone Tracking Caregiver for dementia caregivers: Use of community and healthcare resources. Journal of the American Geriatrics Society, 65(5), 924-930. doi: 10.111/jgs.14684

Turner, A., Eccles, F. J. R., Elvish, R., Simpson, J., \& Keady, J. (2017). The experience of caring for patients with dementia within a general hospital setting: a meta-synthesis of the qualitative literature. Aging \& Mental Health, 21(1), 66-76. Retrieved from http://search.ebscohost.com/login.aspx?direct=true\&db=c8h\&AN=120794069\&site=ehos t-live. doi:10.1080/13607863.2015.1109057

Valcour, V. G., Masaki, K. H., Curb, J., \& Blanchette, P. (2000). The detection of dementia in the primary care setting. Archives of Internal Medicine, 160(19), 2964-2968. Retrieved from http://dx.doi.org/10.1001/archinte.160.19.2964. doi:10.1001/archinte.160.19.2964

Valle, R., Yamada, A. M., \& Barrio, C. (2004). Ethnic differences in social network help-seeking strategies among Latino and Euro-American dementia caregivers. Aging \& Mental Health, 8(6), 535-543. Retrieved from http://search.ebscohost.com.www.libproxy.wvu.edu/login.aspx?direct=true $\& \mathrm{db}=\mathrm{c} 8 \mathrm{~h} \& \mathrm{~A}$ $\underline{\mathrm{N}=2005072450 \& \text { site }=\text { ehost-live }}$

Vecchio, N., Fitzgerald, J. A., Radford, K., \& Fisher, R. (2016). The association between cognitive impairment and community service use patterns in older people living in 
Australia. Health \& Social Care in the Community, 24(3), 321-333.

doi:10.1111/hsc.12212

Walker, L. O., \& Avant, K. C. (2011). Strategies for theory construction in nursing (5th ed.). Boston: Pearson (Prentice Hall).

Wang, Y., Xiao, L. D., Ullah, S., He, G.-P., \& De Bellis, A. (2017). Evaluation of a nurse-led dementia education and knowledge translation programme in primary care: A cluster randomized controlled trial. Nurse Education Today, 49, 1-7. Retrieved from http://www.sciencedirect.com/science/article/pii/S0260691716302544.

doi:https://doi.org/10.1016/j.nedt.2016.10.016

Watson, J. (2008a). Background. In Nursing: The philosophy and science of caring, Revised Edition (pp. 1-12). Boulder, Colorado: University Press of Colorado. Retrieved from http://www.jstor.org/stable/j.ctt1d8h9wn.6

Watson, J. (2008b). Part V. Health, healing, humanity, and heart-centered knowing for Caritas Nursing. In Nursing: The Philosophy and Science of Caring, Revised Edition (pp. 227230). Boulder, Colorado: University Press of Colorado. Retrieved from http://www.jstor.org/stable/j.ctt1d8h9wn.25

Weiss, R. S. (1974). The provisions of social relationships. In Z. Rubin (Ed.), Doing unto others (pp. 17-26). Englewood Cliffs, NJ: Prentice Hall.

Weiss, J., Tumosa, N., Perweiler, E., Bailey, D., Blackwell, E., Forceia, M. A., . . \& Worstell, M. (2017). Workforce Gaps in Dementia Education and Training. Paper presented at the Research Summit on dementia care: Building evidence for services and supports, National Institutes of Health (NIH) campus Bethesda, MD. Retrieved from https://aspe.hhs.gov/system/files/pdf/257826/WorkforceGaps.pdf 
West Virginia Bureau for Public Health Statistics (2015). West Virginia Vital statistics.

Retrieved from http://www.wvdhhr.org/bph/hsc/statserv/VitalStats.asp

West Virginia (WV) Bureau of Senior Services Family Alzheimer's In-Home Respite (FAIR).

Retrieved from http://www.wvseniorservices.gov/HelpatHome/tabid/56/Default.aspx

Whitlatch, C. J., \& Orsulic-Jeras, S. (2018). Meeting the informational, educational, and psychosocial support needs of persons living with dementia and their family caregivers.

The Gerontologist, 58(suppl_1), S58-S73. doi:10.1093/geront/gnx162

Wilks, S. E., \& Croom, B. (2008). Perceived stress and resilience in Alzheimer's disease caregivers: Testing moderation and mediation models of social support. Aging \& Mental Health, 12(3), 357-365. doi: 10.1080/13607860801933323

Wolf, Z. R., \& Heinzer, M. M. (1999). Substruction: Illustrating the connections from research question to analysis. Journal of Professional Nursing, 15(1), 33-37. doi: https://doi.org/10.1016/S8755-7223(99)80023-4

World Health Organization \& Alzheimer's Disease International. (2015). Dementia: A public health priority. Retrieved from http://www.who.int/mental health/publications/dementia report 2012/en/

Ziliak, J. P. (2010). The Appalachian Regional Development Act and economic change [Discussion Paper Series DP2010-14]. University of Kentucky Center for Poverty Research Discussion Paper Series. Retrieved from http://www.ukcpr.org/Publications/DP2010-14.pdf 
Appendix A Abbreviations used throughout text of dissertation in alphabetical order AARP: American Association of Retired Persons (Organization)

ACT: Advanced Caregiver Training (Program)

AD: Alzheimer's Dementia

ADI: Alzheimer's Disease International (Organization)

ADL/IADL: Activities of Daily Living/Instrumental Activities of Daily Living

ADRD: Alzheimer's Disease or Related Dementia

ARC: Appalachian Regional Commission (Organization)

BPSD: Behavioral and Psychological Symptoms of Dementia

BRI-CC: Benjamin Rose Institute Care Consultation

CAFFHO: Community Academic Faith Family Health Organization

CAM: Caregiver Assessment of Managing (Instrument)

CarePRO: Care Partners REACHING OUT (Program)

CES-D: Center for Epidemiologic Studies Depression Scale (Instrument)

CG: Caregiver

CG/CR: dyad Caregiver/Care Recipient dyad

CG/PWD: dyad Caregiver/Person with dementia dyad

CJD: Creutzfeldt-Jakob Disease

CR: Care Recipient (Used interchangeably with Person with Dementia)

CSB: Caregiver Skill Building (Program)

DLB: Dementia with Lewy Bodies

FAIR: Family Alzheimer's In-Home Respite (Program in state of West Virginia)

FCANCC: Family Caregiver Alliance National Center on Caregiving (Organization) 
GED: General Education Development

HCP: Health Care Provider

JHDCNA: Johns Hopkins Dementia Care Needs Assessment (Instrument)

MCI: Mild Cognitive Impairment

NIA: National Institute on Aging (United States Government Organization)

NIH: National Institutes of Health (United States Government Organization)

NINDS: National Institute of Neurological Disorders and Stroke (United States Government

Organization)

NYU-CI: New York University Caregiver Intervention (Program)

PCG-MAI: Philadelphia Geriatric Center Short Length Multi-Level Assessment Instrument

PWD: Person with Dementia (Used interchangeably in paper with Care Recipient)

REACH I AND II: Resources for Enhancing Caregiver Health I (one) and II (two)

REM: Rapid Eye Movement (Stage of Sleep)

SD: Standard Deviation (Statistic)

SHARE: Support, Health, Activities, Resource, and Education (Program)

SPS: Social Provisions Scale (Instrument)

SPS R-10: Social Provisions Scale Revised- 10 item (Instrument)

SPSS: Statistical Package for the Social Sciences (Software Package)

UNA: Unmet Needs Assessment (Instrument)

UNADLT: Unmet Needs Assessment Daily Living Tasks (Subscale in Instrument)

UNAES: Unmet Needs Assessment Emotional Support (Subscale in Instrument)

UNAFC: Unmet Needs Assessment Family Concerns (Subscale in Instrument)

UNAFS: Unmet Needs Assessment Finding Services (Subscale in Instrument) 
UNAHI: Unmet Needs Assessment Health Information (Subscale in Instrument)

UNALA: Unmet Needs Assessment Living Arrangements (Subscale in Instrument)

UNALF: Unmet Needs Assessment Legal and Financial (Subscale in Instrument)

VCP: Victorian Caregiver Program (Program in Australia)

VIF: Variance Inflation Factor (Statistic)

WHO: World Health Organization

WV: West Virginia (State) 
Appendix B Table 1: CG variables used in study Descriptive Statistics

\begin{tabular}{|c|c|c|c|c|c|}
\hline Variable & $\begin{array}{c}\text { Options \& } \\
\text { Ranges }\end{array}$ & Caregiver & $\begin{array}{c}\text { Options \& } \\
\text { Ranges }\end{array}$ & Person with Dementia & $\begin{array}{c}\text { \# no } \\
\text { response or } \\
\text { "prefer not } \\
\text { to say" }\end{array}$ \\
\hline Gender & Female or Male & $\begin{array}{l}\text { Female } n=12 \\
\text { Male } n=31\end{array}$ & Female or Male & $\begin{array}{l}\text { Female } n=15 \\
\text { Male } n=28\end{array}$ & 0 \\
\hline Age & $\begin{array}{l}\text { Fill in age in years } \\
31-90 \text { years range }\end{array}$ & $\begin{array}{l}\text { Mean } 61.78 \text { years }(\mathrm{SD} \\
11.66)\end{array}$ & $\begin{array}{l}\text { Fill in age in years } \\
61-95 \text { years range }\end{array}$ & $\begin{array}{l}\text { Mean } 80.13 \text { years }(\mathrm{SD} \\
9.42)\end{array}$ & $\begin{array}{l}3 \text { CG \& } 3 \\
\text { PWD }\end{array}$ \\
\hline Race & $\begin{array}{l}\text { African American } \\
\text { (Non-Hispanic) } \\
\text { Caucasian (Non- } \\
\text { Hispanic) }\end{array}$ & $\begin{array}{l}1 \\
42 \\
42\end{array}$ & $\begin{array}{l}\text { African American } \\
\text { (Non-Hispanic) } \\
\text { Caucasian (Non- } \\
\text { Hispanic) }\end{array}$ & $\begin{array}{l}1 \\
42\end{array}$ & 0 \\
\hline Marital Status & $\begin{array}{l}\text { Single } \\
\text { In a relationship } \\
\text { Married }\end{array}$ & $\begin{array}{l}1(2.3 \%) \\
2(4.7 \%) \\
31(72.1 \%)\end{array}$ & $\begin{array}{l}\text { Single } \\
\text { Married }\end{array}$ & $\begin{array}{l}3(7 \%) \\
20(46.5) \\
1(2.3 \%)\end{array}$ & 0 \\
\hline
\end{tabular}




\begin{tabular}{|c|c|c|c|c|c|}
\hline Variable & $\begin{array}{c}\text { Options \& } \\
\text { Ranges }\end{array}$ & Caregiver & $\begin{array}{c}\text { Options \& } \\
\text { Ranges }\end{array}$ & Person with Dementia & $\begin{array}{c}\text { \# no } \\
\text { response or } \\
\text { "prefer not } \\
\text { to say" }\end{array}$ \\
\hline & $\begin{array}{l}\text { Divorced or } \\
\text { Separated } \\
\text { Widow/Widower }\end{array}$ & $\begin{array}{l}7(16.3 \%) \\
2(4.7 \%)\end{array}$ & $\begin{array}{l}\text { Divorced or } \\
\text { Separated } \\
\text { Widow/Widower }\end{array}$ & $19(44.2 \%)$ & \\
\hline $\begin{array}{l}\text { Level } \\
\text { Education }\end{array}$ & $\begin{array}{l}<\text { Highschool } \\
\text { Highschool or } \\
\text { GED } \\
\text { Post-High } \\
\text { School/Post GED } \\
\text { College Degree } \\
\text { Professional or } \\
\text { Graduate Degree }\end{array}$ & $\begin{array}{l}1(2.3 \%) \\
13(30.2 \%) \\
5(11.6 \%) \\
13(30.2 \%) \\
11(25.6 \%)\end{array}$ & $\begin{array}{l}<\text { Highschool } \\
\text { Highschool or GED } \\
\text { Post-High } \\
\text { School/Post GED } \\
\text { College Degree } \\
\text { Professional or } \\
\text { Graduate Degree }\end{array}$ & $\begin{array}{l}13(30.2 \%) \\
13(30.2 \%) \\
3(7 \%) \\
4(9.3 \%) \\
9(20.9 \%)\end{array}$ & $1 \mathrm{PWD}$ \\
\hline
\end{tabular}




\begin{tabular}{|c|c|c|c|c|c|}
\hline Variable & $\begin{array}{c}\text { Options \& } \\
\text { Ranges }\end{array}$ & Caregiver & $\begin{array}{c}\text { Options \& } \\
\text { Ranges }\end{array}$ & Person with Dementia & $\begin{array}{c}\text { \# no } \\
\text { response or } \\
\text { "prefer not } \\
\text { to say" }\end{array}$ \\
\hline $\begin{array}{l}\text { Household } \\
\text { Income }\end{array}$ & $\begin{array}{l}<10,000 \\
10,000-39,999 \\
40,000-79,999 \\
80,000-124,999 \\
125,000-150,000 \\
>150,000\end{array}$ & $\begin{array}{l}2(4.7 \%) \\
17(39.5 \%) \\
7(16.3 \%) \\
4(9.3 \%) \\
5(11.6 \%) \\
5(11.6 \%)\end{array}$ & $\begin{array}{l}<10,000 \\
10,000-39,999 \\
40,000-79,999 \\
80,000-124,999 \\
125,000-150,000 \\
>150,000\end{array}$ & $\begin{array}{l}6(14 \%) \\
23(53.5 \%) \\
6(14 \%) \\
1(2.3 \%) \\
1(2.3 \%) \\
2(2.3 \%)\end{array}$ & 3 PWD \\
\hline Owns Home & Yes or No & $\begin{array}{l}\text { Yes } 38(88.4 \%) \\
\text { No } 4(9.3 \%)\end{array}$ & Yes or No & $\begin{array}{l}\text { Yes } 28(65.1 \%) \\
\text { No } 14(32.6 \%)\end{array}$ & $\begin{array}{l}1 \mathrm{CG} \\
1 \mathrm{PWD}\end{array}$ \\
\hline $\begin{array}{l}\text { Advanced Care } \\
\text { Directives }\end{array}$ & Yes or No & $\begin{array}{l}\text { Yes } 23(53.5 \%) \\
\text { No } 17(39.5 \%)\end{array}$ & Yes or No & $\begin{array}{l}\text { Yes } 34(79.1 \%) \\
\text { No } 9(20.9 \%)\end{array}$ & $3 \mathrm{CG}$ \\
\hline $\begin{array}{l}\text { Primary Care } \\
\text { Provider }\end{array}$ & Yes or No & $\begin{array}{l}\text { Yes } 40(93 \%) \\
\text { No } 2(4.7 \%)\end{array}$ & Yes or No & $\begin{array}{l}\text { Yes } 42(97.7) \\
\text { No } 1(2.3 \%)\end{array}$ & $1 \mathrm{CG}$ \\
\hline
\end{tabular}




\begin{tabular}{|c|c|c|c|c|c|}
\hline Variable & $\begin{array}{c}\text { Options \& } \\
\text { Ranges }\end{array}$ & Caregiver & $\begin{array}{c}\text { Options \& } \\
\text { Ranges }\end{array}$ & Person with Dementia & $\begin{array}{c}\text { \# no } \\
\text { response or } \\
\text { "prefer not } \\
\text { to say" }\end{array}$ \\
\hline $\begin{array}{l}\text { Chronic health } \\
\text { conditions treated }\end{array}$ & Yes or No & $\begin{array}{l}\text { Yes } 33(76.7 \%) \\
\text { No } 9(20.9 \%)\end{array}$ & $\begin{array}{l}\text { Yes or No (in } \\
\text { addition to } \\
\text { dementia) }\end{array}$ & $\begin{array}{l}\text { Yes } 36(83.7 \%) \\
\text { No } 7(16.3 \%)\end{array}$ & 0 \\
\hline $\begin{array}{l}\text { Relationship to } \\
\text { PWD }\end{array}$ & $\begin{array}{l}\text { Spouse or Partner } \\
\text { Adult Child } \\
\text { Sibling } \\
\text { Other (no relationship } \\
\text { identified) } \\
\text { Parent (Mother) } \\
\text { Daughter in Law } \\
\text { Niece } \\
\text { Sister in Law }\end{array}$ & $\begin{array}{l}13(30.2 \%) \\
19(44.2 \%) \\
2(4.7 \%) \\
2(4.7 \%) \\
2(4.7 \%) \\
3(7 \%) \\
1(2.3 \%) \\
1(2.3 \%)\end{array}$ & N/A & N/A & 0 \\
\hline
\end{tabular}




\begin{tabular}{|c|c|c|c|c|c|}
\hline Variable & $\begin{array}{c}\text { Options \& } \\
\text { Ranges }\end{array}$ & Caregiver & $\begin{array}{c}\text { Options \& } \\
\text { Ranges }\end{array}$ & Person with Dementia & $\begin{array}{c}\text { \# no } \\
\text { response or } \\
\text { "prefer not } \\
\text { to say" }\end{array}$ \\
\hline $\begin{array}{l}\text { Type of } \\
\text { Dementia }\end{array}$ & $\mathrm{N} / \mathrm{A}$ & $\mathrm{N} / \mathrm{A}$ & $\begin{array}{l}\text { Alzheimers } \\
\text { Vascular } \\
\text { Lewy Bodies } \\
\text { Frontotemporal } \\
\text { Parkinson's' } \\
\text { Mixed } \\
\text { Vascular } \\
\text { Don't Know or } \\
\text { Can't remember } \\
\text { Posterior Cortical } \\
\text { Atrophy } \\
\text { Alzheimers }\end{array}$ & $\begin{array}{l}11(25.6 \%) \\
10(23.3 \%) \\
2(4.7 \%) \\
2(4.7 \%) \\
3(7 \%) \\
1(2.3 \%) \\
6(14 \%) \\
5(11.6 \%) \\
1(2.3 \%) \\
1(2.3 \%)\end{array}$ & $1 \mathrm{PWD}$ \\
\hline
\end{tabular}




\begin{tabular}{|c|c|c|c|c|c|}
\hline Variable & $\begin{array}{c}\text { Options \& } \\
\text { Ranges }\end{array}$ & Caregiver & $\begin{array}{c}\text { Options \& } \\
\text { Ranges }\end{array}$ & Person with Dementia & $\begin{array}{c}\text { \# no } \\
\text { response or } \\
\text { "prefer not } \\
\text { to say" }\end{array}$ \\
\hline & & & $\begin{array}{l}\text { Other (due to } \\
\text { aneurysm, CVA, } \\
\text { infection CNS) }\end{array}$ & & \\
\hline When PWD DX & N/A & N/A & $\begin{array}{l}<1 \text { year } \\
1 \text { year } \\
2 \text { years } \\
3 \text { years } \\
4 \text { years } \\
5 \text { years } \\
6 \text { years } \\
7 \text { years } \\
8 \text { years }\end{array}$ & $\begin{array}{l}2(4.7 \%) \\
7(16.3 \%) \\
4(9.8 \%) \\
8(18.6 \%) \\
4(9.3 \%) \\
5(11.6 \%) \\
3(7 \%) \\
3(7 \%) \\
1(2.3 \%)\end{array}$ & 2 PWD \\
\hline
\end{tabular}




\begin{tabular}{|c|c|c|c|c|c|}
\hline Variable & $\begin{array}{c}\text { Options \& } \\
\text { Ranges }\end{array}$ & Caregiver & $\begin{array}{c}\text { Options \& } \\
\text { Ranges }\end{array}$ & Person with Dementia & $\begin{array}{c}\text { \# no } \\
\text { response or } \\
\text { "prefer not } \\
\text { to say" }\end{array}$ \\
\hline & & & $\begin{array}{l}10 \text { years } \\
11 \text { years } \\
12 \text { years }\end{array}$ & $\begin{array}{l}2(4.7 \%) \\
1(2.3 \%) \\
1(2.3 \%)\end{array}$ & \\
\hline $\begin{array}{l}\text { How long CG } \\
\text { providing care }\end{array}$ & $\begin{array}{l}1 \text { day to } 6 \text { months } \\
1 \text { to } 2 \text { years } \\
3 \text { to } 4 \text { years } \\
5 \text { to } 7 \text { years } \\
8 \text { to } 10 \text { years } \\
\text { Over } 10 \text { years }\end{array}$ & $\begin{array}{l}4(9.3 \%) \\
7(16.3 \%) \\
11(25.6 \%) \\
12(27.9 \%) \\
2(4.7 \%) \\
5(11.6 \%)\end{array}$ & $\mathrm{N} / \mathrm{A}$ & N/A & $2 \mathrm{CG}$ \\
\hline $\begin{array}{l}\text { Hours spend } \\
\text { providing care } \\
\text { per week }\end{array}$ & $\begin{array}{l}<6 \mathrm{hrs} / \text { week } \\
6-10 \mathrm{hrs} / \text { week } \\
11-15 \mathrm{hrs} / \text { week }\end{array}$ & $\begin{array}{l}3(7 \%) \\
2(4.7 \%) \\
6(14 \%)\end{array}$ & N/A & N/A & $1 \mathrm{CG}$ \\
\hline
\end{tabular}




\begin{tabular}{|c|c|c|c|c|c|}
\hline Variable & $\begin{array}{c}\text { Options \& } \\
\text { Ranges }\end{array}$ & Caregiver & $\begin{array}{c}\text { Options \& } \\
\text { Ranges }\end{array}$ & Person with Dementia & $\begin{array}{c}\text { \# no } \\
\text { response or } \\
\text { "prefer not } \\
\text { to say" }\end{array}$ \\
\hline & $\begin{array}{l}16-20 \mathrm{hrs} / \text { week } \\
21-25 \mathrm{hrs} / \text { week } \\
26-30 \mathrm{hrs} / \text { week } \\
31-35 \mathrm{hrs} / \text { week } \\
>40 \mathrm{hrs} / \text { week }\end{array}$ & $\begin{array}{l}3(7 \%) \\
2(4.7 \%) \\
1(2.3 \%) \\
1(2.3 \%) \\
24(55.8 \%)\end{array}$ & & & \\
\hline $\begin{array}{l}\text { Living in same } \\
\text { home as PWD }\end{array}$ & Yes or No & $\begin{array}{l}\text { Yes } 25(58.1 \%) \\
\text { No } 18(41.9 \%)\end{array}$ & N/A & N/A & 0 \\
\hline Where lives & $\begin{array}{l}\text { Private home or } \\
\text { apartment without } \\
\text { service }\end{array}$ & $35(81.4 \%)$ & $\begin{array}{l}\text { Private Home or } \\
\text { apartment without } \\
\text { home care services }\end{array}$ & $25(58.1 \%)$ & 0 \\
\hline
\end{tabular}




\begin{tabular}{|c|c|c|c|c|c|}
\hline Variable & $\begin{array}{c}\text { Options \& } \\
\text { Ranges }\end{array}$ & Caregiver & $\begin{array}{c}\text { Options \& } \\
\text { Ranges }\end{array}$ & Person with Dementia & $\begin{array}{c}\text { \# no } \\
\text { response or } \\
\text { "prefer not } \\
\text { to say" }\end{array}$ \\
\hline & $\begin{array}{l}\text { Private Home or } \\
\text { apartment with } \\
\text { home care services } \\
\text { Nursing Home } \\
\text { (LTC) }\end{array}$ & $\begin{array}{l}(16.3 \%) \\
1(2.3 \%)\end{array}$ & $\begin{array}{l}\text { Private home or } \\
\text { apartment with } \\
\text { home care services } \\
\text { Assisted Living } \\
\text { Nursing Home } \\
\text { (LTC) } \\
\text { Lives with Adult } \\
\text { Daughter }\end{array}$ & $\begin{array}{l}11(25.6 \%) \\
3(7 \%) \\
3(7 \%) \\
1(2.3 \%)\end{array}$ & \\
\hline Who living with & $\begin{array}{l}\text { Alone } \\
\text { Spouse Only } \\
\text { Spouse \& others }\end{array}$ & $\begin{array}{l}4(9.3 \%) \\
23(53.5 \%) \\
6(14 \%) \\
4(9.3 \%)\end{array}$ & N/A & N/A & 0 \\
\hline
\end{tabular}




\begin{tabular}{|c|c|c|c|c|c|}
\hline Variable & $\begin{array}{c}\text { Options \& } \\
\text { Ranges }\end{array}$ & Caregiver & $\begin{array}{c}\text { Options \& } \\
\text { Ranges }\end{array}$ & Person with Dementia & $\begin{array}{c}\text { \# no } \\
\text { response or } \\
\text { "prefer not } \\
\text { to say" }\end{array}$ \\
\hline & $\begin{array}{l}\text { Live with child, no } \\
\text { spouse } \\
\text { Live with others } \\
\text { (not spouse or } \\
\text { child) } \\
\text { Live with parents } \\
\text { Live with child } \\
\text { AND others not } \\
\text { related }\end{array}$ & $\begin{array}{l}1(2.3 \%) \\
4(9.3 \%) \\
1(2.3 \%)\end{array}$ & & & \\
\hline State \& County & $\begin{array}{l}\text { WV (no county } \\
\text { identified) } \\
\text { WV, Cabell Co. }\end{array}$ & $\begin{array}{l}6(14 \%) \\
8(18.6 \%)\end{array}$ & & & 0 \\
\hline
\end{tabular}




\begin{tabular}{|c|c|c|c|c|c|}
\hline Variable & $\begin{array}{c}\text { Options \& } \\
\text { Ranges }\end{array}$ & Caregiver & $\begin{array}{c}\text { Options \& } \\
\text { Ranges }\end{array}$ & Person with Dementia & $\begin{array}{c}\text { \# no } \\
\text { response or } \\
\text { "prefer not } \\
\text { to say" }\end{array}$ \\
\hline $\begin{array}{l}\text { State \& County } \\
\text { continued... }\end{array}$ & $\begin{array}{l}\text { WV, Monongalia } \\
\text { Co. } \\
\text { WV, Tucker Co. } \\
\text { OH, Lawrence Co. } \\
\text { WV, Putnam Co. } \\
\text { WV, Kanawha Co. } \\
\text { WV, Raleigh Co. } \\
\text { WV, Taylor Co. } \\
\text { OH, No county } \\
\text { identified } \\
\text { WV, Wayne Co } \\
\text { WV, Marshall Co. }\end{array}$ & $\begin{array}{l}5(11.6 \%) \\
1(2.3 \%) \\
2(4.7 \%) \\
4(9.3 \%) \\
1(2.3 \%) \\
2(4.7 \%) \\
1(2.3 \%) \\
2(4.7 \%) \\
3(7 \%) \\
1(2.3 \%)\end{array}$ & & & \\
\hline
\end{tabular}




\begin{tabular}{|c|c|c|c|c|c|}
\hline Variable & $\begin{array}{c}\text { Options \& } \\
\text { Ranges }\end{array}$ & Caregiver & $\begin{array}{c}\text { Options \& } \\
\text { Ranges }\end{array}$ & Person with Dementia & $\begin{array}{c}\text { \# no } \\
\text { response or } \\
\text { "prefer not } \\
\text { to say" }\end{array}$ \\
\hline & $\begin{array}{l}\text { PA, Green County } \\
\text { KY, Carter Co. } \\
\text { OH, Gallia Co. }\end{array}$ & $\begin{array}{l}1(2.3 \%) \\
1(2.3 \%) \\
1(2.3 \%)\end{array}$ & & & \\
\hline Primary CG & Yes or No & $\begin{array}{l}\text { Yes } 33(76.7 \%) \\
\text { No } 10(23.3 \%)\end{array}$ & N/A & N/A & 0 \\
\hline $\begin{array}{l}\text { \# of other } \\
\text { persons for } \\
\text { whom } \\
\text { providing care }\end{array}$ & $\begin{array}{l}\text { None } \\
1 \text { other person } \\
2 \text { other persons } \\
3 \text { or more persons }\end{array}$ & $\begin{array}{l}27(62.8 \%) \\
4(9.3 \%) \\
4(9.3 \%) \\
4(9.3 \%)\end{array}$ & N/A & N/A & $3 \mathrm{CG}$ \\
\hline $\begin{array}{l}\text { \# hours paid } \\
\text { help receive per } \\
\text { week }\end{array}$ & $\mathrm{N} / \mathrm{A}$ & $\mathrm{N} / \mathrm{A}$ & $\begin{array}{l}\text { Mean } 12.12 \\
\text { (SD 16.81) } \\
\text { Range 0hrs-58hrs }\end{array}$ & $\begin{array}{cc}\text { HRS } & \text { Frequency }(\%) \\
0 & 21(48.8 \%) \\
2 & 2(4.7 \%) \\
3 & 2(4.7 \%) \\
10 & 2(4.7 \%)\end{array}$ & 0 \\
\hline
\end{tabular}




\begin{tabular}{|c|c|c|c|c|c|c|}
\hline \multirow[t]{2}{*}{ Variable } & $\begin{array}{c}\text { Options \& } \\
\text { Ranges }\end{array}$ & Caregiver & $\begin{array}{c}\text { Options \& } \\
\text { Ranges }\end{array}$ & Perso & Dementia & $\begin{array}{c}\text { \# no } \\
\text { response or } \\
\text { "prefer not } \\
\text { to say" }\end{array}$ \\
\hline & & & & $\begin{array}{l}13 \\
16 \\
21 \\
23 \\
24 \\
28 \\
30 \\
36 \\
40 \\
45 \\
56 \\
58\end{array}$ & $\begin{array}{l}1(2.3 \%) \\
2(4.7 \%) \\
2(4.7 \%) \\
1(2.3 \%) \\
2(4.7 \%) \\
1(2.3 \%) \\
1(2.3 \%) \\
1(2.3 \%) \\
2(4.7 \%) \\
1(2.3 \%) \\
1(2.3 \%) \\
1(2.3 \%)\end{array}$ & \\
\hline
\end{tabular}




\begin{tabular}{|c|c|c|c|c|c|c|}
\hline Variable & $\begin{array}{c}\text { Options \& } \\
\text { Ranges }\end{array}$ & Caregiver & $\begin{array}{c}\text { Options \& } \\
\text { Ranges }\end{array}$ & Pers & with Dementia & $\begin{array}{c}\text { \# no } \\
\text { response or } \\
\text { "prefer not } \\
\text { to say" }\end{array}$ \\
\hline $\begin{array}{l}\text { \# hours unpaid } \\
\text { help receive per } \\
\text { week }\end{array}$ & $\mathrm{N} / \mathrm{A}$ & $\mathrm{N} / \mathrm{A}$ & $\begin{array}{l}\text { Mean } 4.95 \\
(\mathrm{SD} 7.89) \\
\text { Range } 0 \text { hrs-36 hrs }\end{array}$ & $\begin{array}{l}\text { HRS } \\
\begin{array}{l}0 \\
1 \\
1 \\
2 \\
3 \\
4 \\
5 \\
6 \\
8 \\
10 \\
15 \\
20 \\
25 \\
36\end{array}\end{array}$ & $\begin{array}{l}\text { Frequency }(\%) \\
\qquad \begin{array}{l}18(41.9 \%) \\
2(4.7 \%) \\
5(11.6 \%) \\
2(4.7 \%) \\
3(7 \%) \\
2(4.7 \%) \\
1(2.3 \%) \\
2(4.7 \%) \\
2(4.7 \%) \\
2(4.7 \%) \\
2(4.7 \%) \\
1(2.3 \%) \\
1(2.3 \%)\end{array}\end{array}$ & 0 \\
\hline $\begin{array}{l}\text { Employed } \\
\text { outside home }\end{array}$ & $\begin{array}{l}\text { No, Retired } \\
\text { No, Full time } \\
\text { home maker }\end{array}$ & $\begin{array}{l}19(44.2 \%) \\
5(11.6 \%)\end{array}$ & $\mathrm{N} / \mathrm{A}$ & & $\mathrm{N} / \mathrm{A}$ & 0 \\
\hline
\end{tabular}




\begin{tabular}{|c|c|c|c|c|c|}
\hline Variable & $\begin{array}{c}\text { Options \& } \\
\text { Ranges }\end{array}$ & Caregiver & $\begin{array}{c}\text { Options \& } \\
\text { Ranges }\end{array}$ & Person with Dementia & $\begin{array}{c}\text { \# no } \\
\text { response or } \\
\text { "prefer not } \\
\text { to say" }\end{array}$ \\
\hline & $\begin{array}{l}\text { No, I am disabled } \\
\text { Yes, work full } \\
\text { time ( } 36-40 \\
\text { hrs/week) } \\
\text { Yes, work part } \\
\text { time (12-36 } \\
\text { hrs/week) } \\
\text { Yes, part time (4- } \\
12 \text { hrs/week) }\end{array}$ & $\begin{array}{l}1(2.3 \%) \\
16(37.2 \%) \\
1(2.3 \%) \\
1(2.3 \%)\end{array}$ & & & \\
\hline $\begin{array}{l}\text { Faith/spiritual } \\
\text { beliefs affecting } \\
\text { life decisions }\end{array}$ & $\begin{array}{l}\text { Yes } \\
\text { No }\end{array}$ & $\begin{array}{l}35(81.4 \%) \\
8(18.6 \%)\end{array}$ & $\mathrm{N} / \mathrm{A}$ & N/A & 0 \\
\hline
\end{tabular}




\begin{tabular}{|c|c|c|c|c|c|}
\hline Variable & $\begin{array}{c}\text { Options \& } \\
\text { Ranges }\end{array}$ & Caregiver & $\begin{array}{c}\text { Options \& } \\
\text { Ranges }\end{array}$ & Person with Dementia & $\begin{array}{c}\text { \# no } \\
\text { response or } \\
\text { "prefer not } \\
\text { to say" }\end{array}$ \\
\hline $\begin{array}{l}\text { Regular } \\
\text { attendance } \\
\text { faith/spiritual } \\
\text { based meetings }\end{array}$ & $\begin{array}{l}\text { Yes } \\
\text { No }\end{array}$ & $\begin{array}{l}19(44.2 \%) \\
24(55.8 \%)\end{array}$ & $\mathrm{N} / \mathrm{A}$ & $\mathrm{N} / \mathrm{A}$ & 0 \\
\hline
\end{tabular}


Appendix C Figure 1: Andersen's Behavioral Model of Service Use

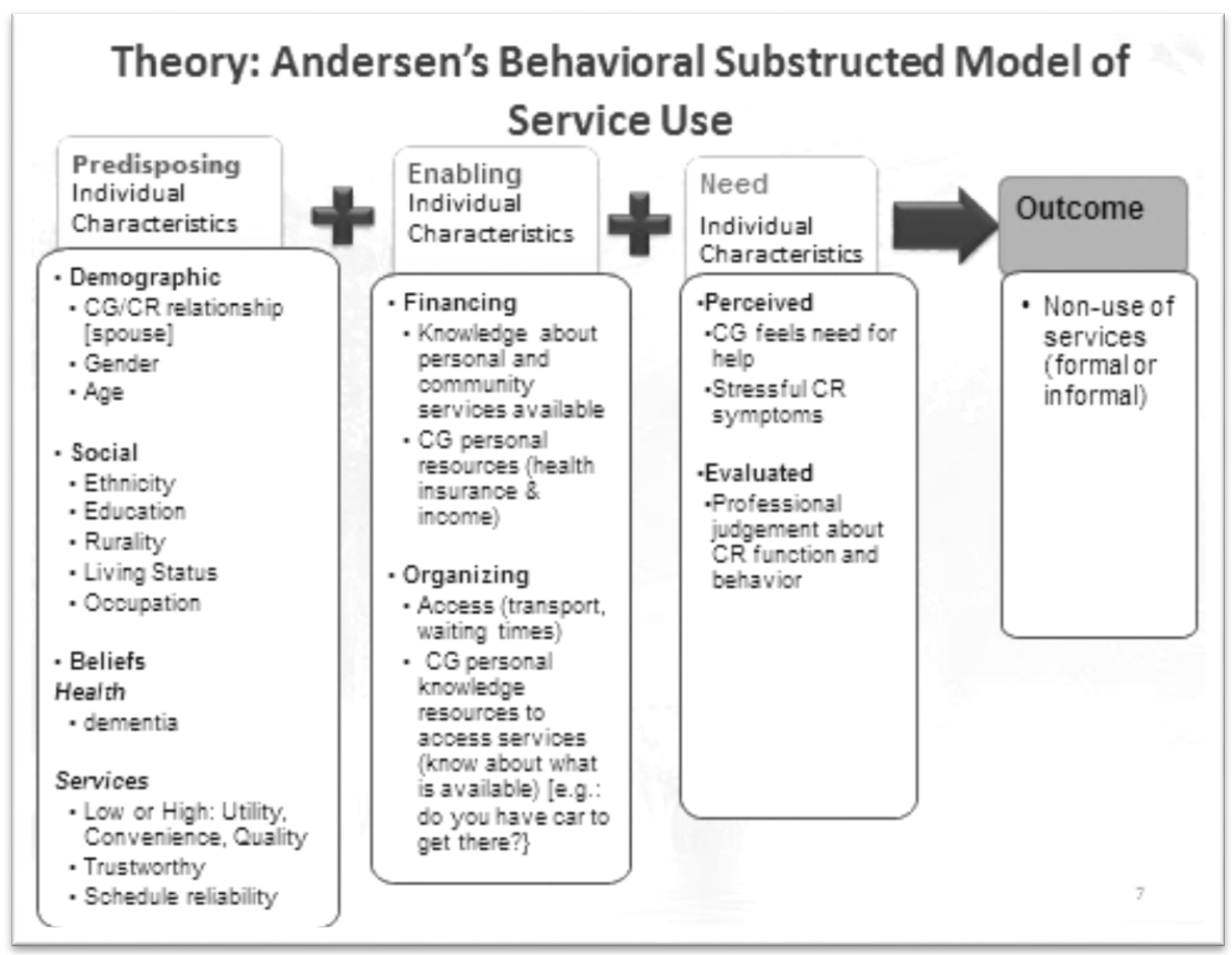

Extrapolated from Phase 5 of Andersen's Behavioral Model of Health Service Use (Andersen, 2008, p. 651) by Kristina M. Childers 
Appendix C Figure 2: Andersen's Behavioral Substructed Model of Service Use with variables from this Study (following data collection)

Theory: Andersen's Behavioral Substructed Model of Service Use

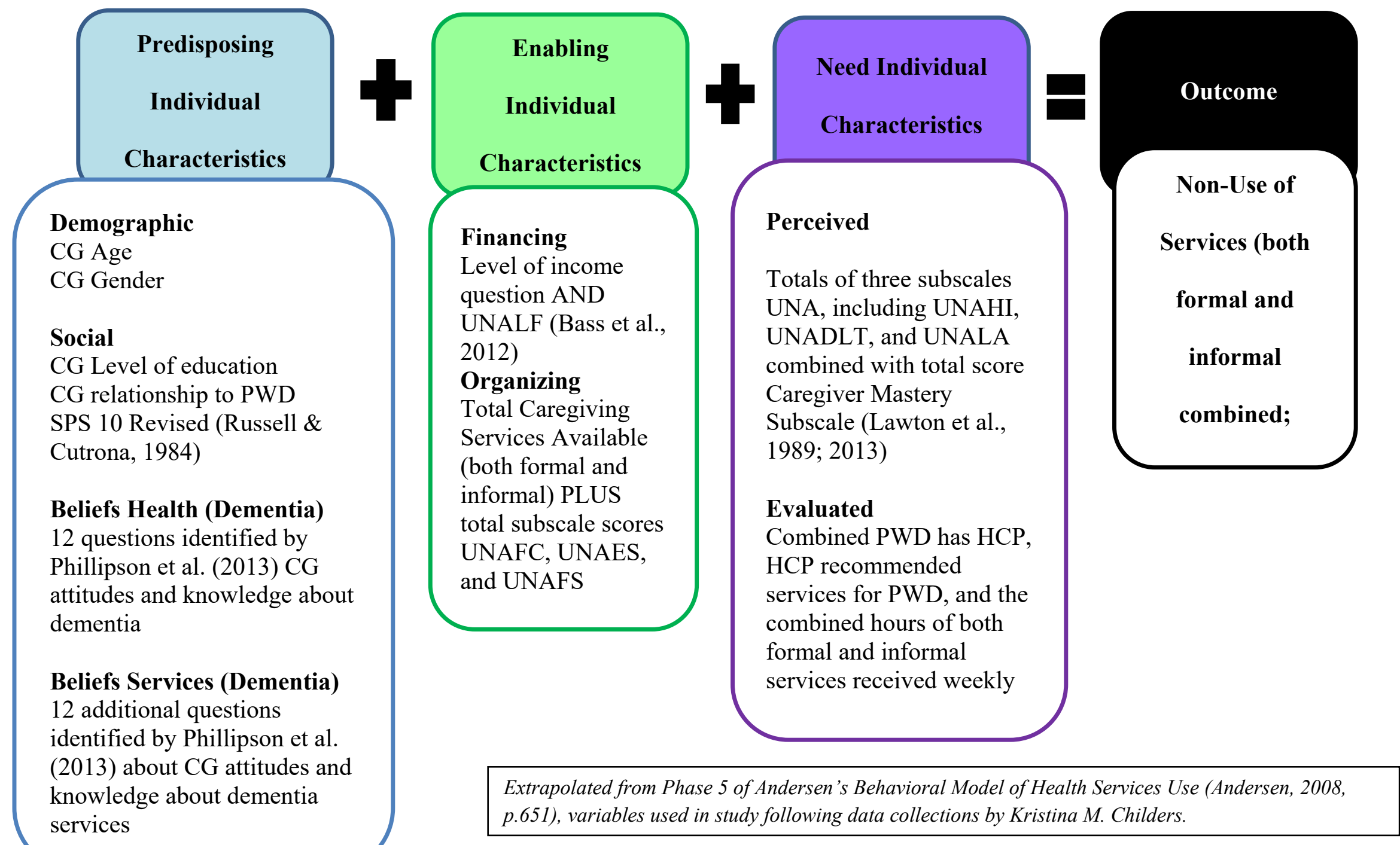


Appendix D Table 2 Inclusion and Exclusion Literature Search

Databases used: Academic Search Complete, Ageline, CINAHL with Full text, Education research complete, Health Source: Nursing/Academic Edition, MEDLINE, PsycArticles, PsycINFO, Women's studies. All databases via West Virginia University Libraries

Keywords: rural, rural spousal caregivers, rural caregivers, caregiver, dementia patient, dementia, Appalachia, Appalachian

\begin{tabular}{|c|c|}
\hline Inclusion Criteria & Exclusion Criteria \\
\hline English & Language other than English \\
\hline Peer reviewed journals & Non peer review \\
\hline Research article & Editorials \\
\hline Human subjects & Non-human subjects \\
\hline $\begin{array}{l}\text { Adulthood (age } 18 \text { years and } \\
\text { older) }\end{array}$ & Infants, children (under 18 years of age) \\
\hline $\begin{array}{l}\text { Informal caregivers of patients } \\
\text { with dementia }\end{array}$ & $\begin{array}{l}\text { Caregivers of patients with illnesses other } \\
\text { than dementia }\end{array}$ \\
\hline $\begin{array}{l}\text { Rural caregivers in the United } \\
\text { States }\end{array}$ & Rural caregivers outside the United States \\
\hline $\begin{array}{l}\text { Dates: January } 2000 \text { through } \\
\text { August } 2011\end{array}$ & Those in a long-term care facility \\
\hline
\end{tabular}

*Original Search for Appalachian, caregiver, dementia yielded zero results. 
Appendix E Table 3: Andersen's Model, Study Variables, and Measures Literature Review and Survey

\begin{tabular}{|c|c|c|c|c|c|c|}
\hline $\begin{array}{c}\text { Andersen's } \\
\text { Behavioral } \\
\text { Model of } \\
\text { Health Service } \\
\text { Use }\end{array}$ & Study Variables & Measure & $\begin{array}{c}\text { Reliability \& } \\
\text { Validity }\end{array}$ & $\begin{array}{c}\text { Study } \\
\text { Citation }\end{array}$ & $\begin{array}{l}\text { Survey } \\
\text { (page \#) }\end{array}$ & $\begin{array}{c}\text { Not Used \& } \\
\text { Reason }\end{array}$ \\
\hline \multicolumn{7}{|c|}{ Predisposing Characteristics } \\
\hline Demographics & $\begin{array}{l}\text { Demographic } \\
\text { variables } \\
\text { CG/CR relationship } \\
\text { Gender } \\
\text { Age }\end{array}$ & $\begin{array}{l}\text { Relationship } \\
\text { CG/CR } \\
\text { Gender CG/CR } \\
\text { Age CG/CR } \\
\text { When was CR dx? } \\
\text { How long have } \\
\text { you been } \\
\text { providing care? } \\
\text { How many hours } \\
\text { of care per week } \\
\text { do you provide? } \\
\text { Do you provide } \\
\text { care for another } \\
\text { person in addition } \\
\text { to the CR (child, } \\
\text { another adult, } \\
\text { etc.)? } \\
\text { CRS }\end{array}$ & $\begin{array}{l}\text { Not } \\
\text { Applicable } \\
\text { (N/A) }\end{array}$ & $\begin{array}{l}\text { Phillipson et } \\
\text { al. (2013) } \\
\text { Suggested } \\
\text { Questions } \\
\text { added from } \\
\text { CRN:BR }\end{array}$ & $\begin{array}{l}\text { P.2 Relationship } \\
\text { to PWD } \\
\text { P.1 gender, age, } \\
\text { race/ethnicity } \\
\text { CRN:BR- p.3 } \\
\text { when diagnosed, } \\
\text { how long } \\
\text { providing care, \# } \\
\text { hours per week } \\
\text { provide care } \\
\text { P.4 to 5- How } \\
\text { many other } \\
\text { people do you } \\
\text { provide unpaid } \\
\text { care or assistance } \\
\text { in addition to } \\
\text { PWD }\end{array}$ & $\begin{array}{l}\text { CRS Not } \\
\text { used as } \\
\text { questions } \\
\text { covered in }\end{array}$ \\
\hline
\end{tabular}




\begin{tabular}{|c|c|c|c|c|c|c|}
\hline $\begin{array}{c}\text { Andersen's } \\
\text { Behavioral } \\
\text { Model of } \\
\text { Health Service } \\
\text { Use }\end{array}$ & Study Variables & Measure & $\begin{array}{c}\text { Reliability \& } \\
\text { Validity }\end{array}$ & $\begin{array}{c}\text { Study } \\
\text { Citation }\end{array}$ & $\begin{array}{l}\text { Survey } \\
\text { (page \#) }\end{array}$ & $\begin{array}{c}\text { Not Used \& } \\
\text { Reason }\end{array}$ \\
\hline & & & & & & $\begin{array}{l}\text { other } \\
\text { instruments }\end{array}$ \\
\hline Social & $\begin{array}{l}\text { Ethnicity/Race } \\
\text { Education } \\
\text { Rurality } \\
\text { Living status } \\
\text { Occupation } \\
\text { Language spoken at } \\
\text { home (other than } \\
\text { English) } \\
\text { SPS 10 R (Russell \& } \\
\text { Cutrona, 1984) }\end{array}$ & $\begin{array}{l}\text { Ethnicity/Race } \\
\text { CG/CR } \\
\text { Ed. Level of } \\
\text { CG/CR } \\
\text { Rurality CG/CR } \\
\text { Do you live with } \\
\text { CR? } \\
\text { Occupation } \\
\text { CG/CR }\end{array}$ & $\begin{array}{l}\text { N/A } \\
\text { SPS } \alpha=0.85\end{array}$ & $\begin{array}{l}\text { Phillipson, et } \\
\text { al. (2014) } \\
\text { Phillipson et } \\
\text { al. (2013) } \\
\\
\text { Russell and } \\
\text { Cutrona } \\
\text { (1984) }\end{array}$ & $\begin{array}{l}\text { P.1-Race or } \\
\text { ethnicity; } \\
\text { education } \\
\text { P.3 Living in } \\
\text { same home as } \\
\text { CR or PWD } \\
\text { P.1- all } \\
\text { respondents } \\
\text { spoke English as } \\
\text { primary } \\
\text { language } \\
\text { P.5 "Are you } \\
\text { employed } \\
\text { outside the } \\
\text { home?" }\end{array}$ & $\begin{array}{l}\text { Rurality- all } \\
\text { counties } \\
\text { identified as } \\
\text { "Appalachia" } \\
\text { are } \\
\text { considered } \\
\text { rural per } \\
\text { ARC } \\
\text { Occupation } \\
\text { not used as } \\
\text { there was } \\
\text { already } \\
\text { question } \\
\text { regarding } \\
\text { employed } \\
\text { outside } \\
\text { home, } \\
\text { retired, } \\
\text { disabled part- } \\
\text { time or full } \\
\text { time. }\end{array}$ \\
\hline
\end{tabular}




\begin{tabular}{|c|c|c|c|c|c|c|}
\hline $\begin{array}{c}\text { Andersen's } \\
\text { Behavioral } \\
\text { Model of } \\
\text { Health Service } \\
\text { Use }\end{array}$ & Study Variables & Measure & $\begin{array}{c}\text { Reliability \& } \\
\text { Validity }\end{array}$ & $\begin{array}{c}\text { Study } \\
\text { Citation }\end{array}$ & $\begin{array}{l}\text { Survey } \\
\text { (page \#) }\end{array}$ & $\begin{array}{c}\text { Not Used \& } \\
\text { Reason }\end{array}$ \\
\hline $\begin{array}{l}\text { Beliefs Health } \\
\text { (Dementia) }\end{array}$ & $\begin{array}{l}\text { Health belief } \\
\text { (Dementia)statements } \\
\text { including beliefs } \\
\text { about dementia } \\
\text { STATEMENTS: } \\
\text { "embarrassment to be } \\
\text { in public with CR" } \\
\text { "concern about } \\
\text { guests in home" } \\
\text { [stigma] } \\
\text { Four treatment } \\
\text { effectiveness for } \\
\text { dementia belief } \\
\text { statements }\end{array}$ & $\begin{array}{l}\text { Questions about } \\
\text { stigma, dementia, } \\
\text { treatment beliefs }\end{array}$ & $\begin{array}{l}\text { Stigma } \alpha= \\
0.64\end{array}$ & $\begin{array}{l}\text { Phillipson et } \\
\text { al. (2013) }\end{array}$ & $\begin{array}{l}\text { P. } 11 \text { bottom of } \\
\text { page to page } 12 \text {, } \\
\text { last question } \\
\text { regarding beliefs } \\
\text { (dementia) is " } \mathrm{A} \\
\text { variety of } \\
\text { treatments are } \\
\text { available for } \\
\text { dementia" }\end{array}$ & \\
\hline $\begin{array}{l}\text { Beliefs } \\
\text { (Service) }\end{array}$ & $\begin{array}{l}\text { Low or High utility } \\
\text { Low or High } \\
\text { convenience } \\
\text { Low or High Quality } \\
\text { Trustworthy } \\
\text { Schedule reliability } \\
\text { of service } \\
\text { Willing to pay for } \\
\text { service (CRN:BR) }\end{array}$ & $\begin{array}{l}\text { Phillipson et al. } \\
2013-12 \text { questions } \\
\text { about services } \\
\text { (dementia) } \\
\text { including } \\
\text { government, } \\
\text { access to services, } \\
\text { value of service } \\
\text { use, or potential } \\
\text { negative outcome }\end{array}$ & $\alpha=0.66$ & $\begin{array}{l}\text { Phillipson et } \\
\text { al. (2013) } \\
\text { CRN: BR }\end{array}$ & $\begin{array}{l}\text { P. } 12 \text { beginning } \\
\text { with "The } \\
\text { government } \\
\text { should provide } \\
\text { more money for } \\
\text { respite programs } \\
\text { and services to } \\
\text { caregivers." }\end{array}$ & $\begin{array}{l}\text { CRN:BR } \\
\text { questions } \\
\text { were similar } \\
\text { to Phillipson } \\
\text { et al. (2013) } \\
\text { The "Willing } \\
\text { to pay for } \\
\text { service" was } \\
\text { not utilized to } \\
\text { limit }\end{array}$ \\
\hline
\end{tabular}




\begin{tabular}{|c|c|c|c|c|c|c|}
\hline $\begin{array}{c}\text { Andersen's } \\
\text { Behavioral } \\
\text { Model of } \\
\text { Health Service } \\
\text { Use }\end{array}$ & Study Variables & Measure & $\begin{array}{c}\text { Reliability \& } \\
\text { Validity }\end{array}$ & $\begin{array}{c}\text { Study } \\
\text { Citation }\end{array}$ & $\begin{array}{l}\text { Survey } \\
\text { (page \#) }\end{array}$ & $\begin{array}{c}\text { Not Used \& } \\
\text { Reason }\end{array}$ \\
\hline & $\begin{array}{l}\text { CG with "duty to } \\
\text { care" } \\
\text { Two questions r/t } \\
\text { belief statements } \\
\text { about government } \\
\text { role dementia care }\end{array}$ & $\begin{array}{l}\text { for PWD if use } \\
\text { service } \\
\text { Questions CRN: } \\
\text { BR }\end{array}$ & & & & $\begin{array}{l}\text { respondent } \\
\text { burden. }\end{array}$ \\
\hline \multicolumn{7}{|c|}{ Enabling Characteristics } \\
\hline Financing & $\begin{array}{l}\text { Personal \& } \\
\text { Community Level- } \\
\text { staff \& services } \\
\text { where people live, } \\
\text { and work Have } \\
\text { personal knowledge } \\
\text { and resources (health } \\
\text { insurance \& income) } \\
\text { CG income } \\
\text { Subscale from UNA } \\
\text { (Bass et al., 2012) } \\
\text { UNALF }\end{array}$ & $\begin{array}{l}\text { "Unmet Needs" } \\
\text { Scale Financial } \\
\text { and Legal=4 } \\
\text { questions } \\
\text { answered "yes" or } \\
\text { "no" as to CG } \\
\text { needing or wanting } \\
\text { help or assistance } \\
\text { with the } \\
\text { instrument topic- }\end{array}$ & 更 & $\begin{array}{l}\text { Phillipson et } \\
\text { al. (2014) } \\
\text { Phillipson et } \\
\text { al. (2013) }\end{array}$ & $\begin{array}{l}\text { P.8 UNALF dd) } \\
\text { through jj) }\end{array}$ & $\begin{array}{l}\text { The } \\
\text { Phillipson et } \\
\text { al. (2014) } \\
\text { questions not } \\
\text { included } \\
\text { about } \\
\text { community } \\
\text { level staff, } \\
\text { health } \\
\text { insurance not } \\
\text { asked as used } \\
\text { UNALF and } \\
\text { CG income. } \\
\text { Also, later } \\
\text { used } \\
\text { Caregiving }\end{array}$ \\
\hline
\end{tabular}




\begin{tabular}{|c|c|c|c|c|c|c|}
\hline $\begin{array}{c}\text { Andersen's } \\
\text { Behavioral } \\
\text { Model of } \\
\text { Health Service } \\
\text { Use }\end{array}$ & Study Variables & Measure & $\begin{array}{c}\text { Reliability \& } \\
\text { Validity }\end{array}$ & $\begin{array}{c}\text { Study } \\
\text { Citation }\end{array}$ & $\begin{array}{l}\text { Survey } \\
\text { (page \#) }\end{array}$ & $\begin{array}{c}\text { Not Used \& } \\
\text { Reason }\end{array}$ \\
\hline & & & & & & $\begin{array}{l}\text { service } \\
\text { available } \\
\text { which } \\
\text { identified } \\
\text { which } \\
\text { services CG } \\
\text { had available } \\
\text { and which } \\
\text { ones he/she } \\
\text { used. }\end{array}$ \\
\hline Organizing & $\begin{array}{l}\text { Access (transport, } \\
\text { waiting times) } \\
\text { CG personal } \\
\text { knowledge of } \\
\text { resources to access } \\
\text { services (know about } \\
\text { what is available) } \\
\text { \{e.g.: do have you } \\
\text { have car?\} } \\
\text { CG informal } \\
\text { support? }\end{array}$ & $\begin{array}{l}\text { Do you drive? } \\
\text { Do you have a } \\
\text { dependable car } \\
\text { (source of } \\
\text { transportation) } \\
\text { available to you } \\
\text { most days? }\end{array}$ & $\begin{array}{l}\text { N/A } \\
\text { N/A }\end{array}$ & $\begin{array}{l}\text { Suggest } \\
\text { Questions } \\
\text { added from } \\
\text { CRN:BR }\end{array}$ & $\begin{array}{l}\text { P. } 7 \text { UNAFC } \\
\text { Questions a-g } \\
\text { P. } 8 \text { UNAES } \\
\text { Questions kk-nn } \\
\text { P. } 8 \text { UNAFS } \\
\text { Questions oo-tt } \\
\\
\text { P.9-10 Formal } \\
\text { services }\end{array}$ & $\begin{array}{l}\text { Did not use } \\
\text { CRN:BR as } \\
\text { UNAFC, } \\
\text { UNAES, } \\
\text { UNAFS and } \\
\text { Caregiving } \\
\text { services } \\
\text { available } \\
\text { incorporated } \\
\text { the } \\
\text { information }\end{array}$ \\
\hline
\end{tabular}




\begin{tabular}{|c|c|c|c|c|c|c|}
\hline $\begin{array}{c}\text { Andersen's } \\
\text { Behavioral } \\
\text { Model of } \\
\text { Health Service } \\
\text { Use }\end{array}$ & Study Variables & Measure & $\begin{array}{c}\text { Reliability \& } \\
\text { Validity }\end{array}$ & $\begin{array}{c}\text { Study } \\
\text { Citation }\end{array}$ & $\begin{array}{l}\text { Survey } \\
\text { (page \#) }\end{array}$ & $\begin{array}{c}\text { Not Used \& } \\
\text { Reason }\end{array}$ \\
\hline & $\begin{array}{l}\text { Caregiver having } \\
\text { social support } \\
\text { network to rely } \\
\text { upon? } \\
39 \text { Services used to } \\
\text { create Vignettes } \\
\text { Caregiving Services } \\
\text { Available (both } \\
\text { Formal and Informal) }\end{array}$ & $\begin{array}{l}\text { "Unmet Needs" } \\
\text { Scale } \\
\text { UNAFC } \\
\text { UNAES } \\
\text { UNAFS } \\
\text { Do you have } \\
\text { family/friend who } \\
\text { assists you in CG } \\
\text { role? (y/n) } \\
\text { Vignettes } \\
\text { Lists of services } \\
\text { from article } \\
\text { including meals } \\
\text { delivered, home } \\
\text { health care, etc. }\end{array}$ & $\alpha=0.92$ & $\begin{array}{l}\text { Bass et } \\
\text { al.(2003), Bass } \\
\text { et al. (2012) } \\
\text { Robinson et al. } \\
(2005 ; \text { 2013) } \\
\text { Ploeg et al. } \\
\text { (2009) } \\
\text { Phillipson et } \\
\text { al. (2014) }\end{array}$ & $\begin{array}{l}\text { available and do } \\
\text { you use service } \\
\text { currently, do not } \\
\text { use service, used } \\
\text { in past but no } \\
\text { longer use } \\
\text { P.10 Informal } \\
\text { Services } \\
\text { Available/Used } \\
\text { Begin after } \\
\text { "Veteran } \\
\text { Services" with } \\
\text { "Help with } \\
\text { transportation..." }\end{array}$ & $\begin{array}{l}\text { Did not use } \\
\text { Ploeg et } \\
\text { al.(2009) as } \\
\text { similar } \\
\text { questions } \\
\text { covered as } \\
\text { above so } \\
\text { vignettes not } \\
\text { needed } \\
\text { (duplicated } \\
\text { material) } \\
\text { Did not use } \\
\text { Robinson et } \\
\text { al. (2005, } \\
2013) \text { as } \\
\text { materials } \\
\text { were already } \\
\text { addressed by } \\
\text { Predisposing } \\
\text { SPS } 10 \text { R }\end{array}$ \\
\hline
\end{tabular}




\begin{tabular}{|c|c|c|c|c|c|c|}
\hline $\begin{array}{c}\text { Andersen's } \\
\text { Behavioral } \\
\text { Model of } \\
\text { Health Service } \\
\text { Use }\end{array}$ & Study Variables & Measure & $\begin{array}{c}\text { Reliability \& } \\
\text { Validity }\end{array}$ & $\begin{array}{c}\text { Study } \\
\text { Citation }\end{array}$ & $\begin{array}{l}\text { Survey } \\
\text { (page \#) }\end{array}$ & $\begin{array}{c}\text { Not Used \& } \\
\text { Reason }\end{array}$ \\
\hline \multicolumn{7}{|c|}{ Need Characteristics } \\
\hline Perceived & $\begin{array}{l}\text { CG feels need for } \\
\text { help } \\
\text { Stressful CR } \\
\text { symptoms } \\
\text { CR needs asst. with } \\
\text { ADLs? } \\
\text { CR wandering? (y/n) } \\
\text { CR behavior } \\
\text { problems? } \\
\text { CG burden } \\
\text { CG depressive } \\
\text { symptoms } \\
\text { Effect of respite on } \\
\text { CG \& CR (stress } \\
\text { level, health } \\
\text { improved, benefits, } \\
\text { frequency, length, } \\
\text { cost of use) and } 2 \\
\text { open ended questions } \\
\text { from researchers } \\
\text { about effects of } \\
\text { respite }\end{array}$ & $\begin{array}{l}\text { PGC-MAI } \\
\text { currently known as } \\
\text { CAM } \\
\text { ZBI (4 item) } \\
\text { [Bédard et al., } \\
2001 \text { as cited in } \\
\text { Phillipson et al.) } \\
\text { CES-D screener } \\
\text { (Radloff, 1977)- } \\
\text { from Phillipson et } \\
\text { al. (2013) } \\
\text { CPRSS }\end{array}$ & $\begin{array}{l}\text { PGC-MAI } \\
\text { (CAM) } \\
\alpha=0.67-0.76\end{array}$ & $\begin{array}{l}\text { Robinson, } \\
\text { Buckwalter et } \\
\text { al. (2005) } \\
\text { developed by } \\
\text { Lawton et al., } \\
1993 \text { as cited } \\
\text { in Robinson, } \\
\text { Buckwalter et } \\
\text { al. (2005) } \\
\text { Phillipson et } \\
\text { al. (2013) }\end{array}$ & $\begin{array}{l}\text { Caregiver } \\
\text { Assessment of } \\
\text { Mastery } \\
\text { Subscale (CAM) } \\
\text { p. } 11 \text { (top table } \\
\text { on page) }\end{array}$ & $\begin{array}{l}\text { ZBI, CES-D, } \\
\text { CPRSS Not } \\
\text { used due to } \\
\text { respondent } \\
\text { burden and } \\
\text { CAM } \\
\text { assessed CG } \\
\text { feelings } \\
\text { about how } \\
\text { CG never to } \\
\text { nearly always } \\
\text { feels guilt, } \\
\text { uncertainty, } \\
\text { need to do } \\
\text { more, or } \\
\text { could do a } \\
\text { better job for } \\
\text { PWD } \\
\text { Did not use } \\
\text { respite } \\
\text { questions }\end{array}$ \\
\hline
\end{tabular}




\begin{tabular}{|c|c|c|c|c|c|c|}
\hline $\begin{array}{c}\text { Andersen's } \\
\text { Behavioral } \\
\text { Model of } \\
\text { Health Service } \\
\text { Use }\end{array}$ & Study Variables & Measure & $\begin{array}{c}\text { Reliability \& } \\
\text { Validity }\end{array}$ & $\begin{array}{c}\text { Study } \\
\text { Citation }\end{array}$ & $\begin{array}{l}\text { Survey } \\
\text { (page \#) }\end{array}$ & $\begin{array}{l}\text { Not Used \& } \\
\text { Reason }\end{array}$ \\
\hline & $\begin{array}{l}\text { UNAHI, UNADT, } \\
\text { UNALA }\end{array}$ & $\begin{array}{l}\text { What is effect of } \\
\text { respite on } \mathrm{CG} / \mathrm{CR} \\
\text { health? }\end{array}$ & & $\begin{array}{l}\text { Bass et al. } \\
(2003 ; 2012)\end{array}$ & $\begin{array}{l}\text { P.7 UNAHI } \\
\text { questions h-v } \\
\text { P.7 UNADT } \\
\text { questions w-aa } \\
\text { P. } 8 \text { UNALA } \\
\text { questions uu-ww }\end{array}$ & $\begin{array}{l}\text { because not } \\
\text { all CG used } \\
\text { respite } \\
\text { Did ask } \\
\text { question } \\
\text { about CG and } \\
\text { PWD other } \\
\text { health } \\
\text { conditions P. } \\
2\end{array}$ \\
\hline Evaluated & $\begin{array}{l}\text { Professional } \\
\text { judgement about CR } \\
\text { function and behavior } \\
\text { JHDCNA }\end{array}$ & $\begin{array}{l}\text { PCG-MAI, a.k.a. } \\
\text { CAM }\end{array}$ & $\begin{array}{l}\text { PGC-MAI } \\
\text { (CAM) } \\
\alpha=0.67-0.76\end{array}$ & $\begin{array}{l}\text { Lawton et al. } \\
\text { (1993) } \\
\text { Johnston et al. } \\
\text { (2011) and } \\
\text { Black et al. } \\
\text { (2013) } \\
\text { Reinhard et al. } \\
\text { (2008) } \\
\text { Alzheimer's } \\
\text { Association } \\
\text { and National } \\
\text { Alliance for }\end{array}$ & $\begin{array}{l}\text { P.2 "Is there a } \\
\text { primary health } \\
\text { care provider?" } \\
\text { for both CG and } \\
\text { CR } \\
\text { P.2 "Did your } \\
\text { health care } \\
\text { provider } \\
\text { recommend } \\
\text { services for the } \\
\text { PWD?" } \\
\text { P.5 two } \\
\text { questions about } \\
\text { hours per week }\end{array}$ & $\begin{array}{l}\text { Did not use } \\
\text { CAM here as } \\
\text { it was closer } \\
\text { to Perceived } \\
\text { Need and due } \\
\text { to respondent } \\
\text { burden, did } \\
\text { not include } \\
\text { professional } \\
\text { judgement } \\
\text { other than } \\
\text { diagnosis of a } \\
\text { dementia by } \\
\text { HCP. }\end{array}$ \\
\hline
\end{tabular}




\begin{tabular}{|c|c|c|c|c|c|c|}
\hline $\begin{array}{c}\text { Andersen's } \\
\text { Behavioral } \\
\text { Model of } \\
\text { Health Service } \\
\text { Use }\end{array}$ & Study Variables & Measure & $\begin{array}{c}\text { Reliability \& } \\
\text { Validity }\end{array}$ & $\begin{array}{c}\text { Study } \\
\text { Citation }\end{array}$ & $\begin{array}{l}\text { Survey } \\
\text { (page \#) }\end{array}$ & $\begin{array}{c}\text { Not Used \& } \\
\text { Reason }\end{array}$ \\
\hline & $\begin{array}{l}\text { PWD had Health } \\
\text { Care Provider (HCP) } \\
\text { HCP recommended } \\
\text { services } \\
\text { Total Weekly hours } \\
\text { of formal and } \\
\text { informal help } \\
\text { received per week }\end{array}$ & & & $\begin{array}{l}\text { Family } \\
\text { Caregivers } \\
(2004) \\
\text { Family } \\
\text { Caregiver } \\
\text { Alliance } \\
(2014)\end{array}$ & $\begin{array}{l}\text { paid and unpaid } \\
\text { help received for } \\
\text { PWD. }\end{array}$ & $\begin{array}{l}\text { Did not use } \\
\text { JHDCNA as } \\
\text { It was more } \\
\text { for interview } \\
\text { in home and } \\
\text { to follow } \\
\text { interview } \\
\text { with more } \\
\text { assessment, } \\
\text { which did not } \\
\text { lend itself to } \\
\text { the scope of } \\
\text { this study. }\end{array}$ \\
\hline \multicolumn{7}{|c|}{ Non-use of Services } \\
\hline $\begin{array}{l}\text { Formal and } \\
\text { Informal }\end{array}$ & $\begin{array}{l}\text { Formal (paid) and } \\
\text { informal services } \\
\text { (unpaid) } \\
\text { (e.g.: home services, } \\
\text { day care, support } \\
\text { group, help with } \\
\text { transportation, etc.) } \\
\text { List of services used } \\
\text { within } 12 \text { months }\end{array}$ & $\begin{array}{l}\text { Gitlin's questions } \\
\text { from REACH I } \\
\text { (List of eight } \\
\text { formal and six } \\
\text { informal services } \\
\text { used in past month } \\
\text { yes/no) }\end{array}$ & $\mathrm{N} / \mathrm{A}$ & $\begin{array}{l}\text { Gitlin et al. } \\
(2003) \\
\text { Phillipson et } \\
\text { al. (2014) } \\
\text { Phillipson et } \\
\text { al. (2013) }\end{array}$ & $\begin{array}{l}\text { P.8 Formal } \\
\text { services begin at } \\
\text { the bottom of } \\
\text { page } 8 \text { and } \\
\text { continue to } \\
\text { Veteran Services } \\
\text { P.9 Informal } \\
\text { Services begin } \\
\text { following } \\
\text { "Veteran } \\
\text { Services" and } \\
\text { continue to }\end{array}$ & $\begin{array}{l}\text { Did not use } \\
\text { O'Connell } \\
\text { questions as } \\
\text { they } \\
\text { compared } \\
\text { very closely } \\
\text { with } \\
\text { Phillipson et } \\
\text { al. (2013) } \\
\text { and VCP } \\
\text { were similar. } \\
\text { Had to limit }\end{array}$ \\
\hline
\end{tabular}




\begin{tabular}{|c|c|c|c|c|c|c|}
\hline $\begin{array}{c}\text { Andersen's } \\
\text { Behavioral } \\
\text { Model of } \\
\text { Health Service } \\
\text { Use }\end{array}$ & Study Variables & Measure & $\begin{array}{c}\text { Reliability \& } \\
\text { Validity }\end{array}$ & $\begin{array}{c}\text { Study } \\
\text { Citation }\end{array}$ & $\begin{array}{l}\text { Survey } \\
\text { (page \#) }\end{array}$ & $\begin{array}{c}\text { Not Used \& } \\
\text { Reason }\end{array}$ \\
\hline & $\begin{array}{l}\text { la } \\
\text { VCP-multiple } \\
\text { measures about using } \\
\text { services in Australia }\end{array}$ & $\begin{array}{l}\text { Phillipson et al. } \\
(2013,2014) \text { list of } \\
\text { services } \\
\text { CRS }\end{array}$ & & $\begin{array}{l}\text { O'Connell et } \\
\text { al. (2012) } \\
\text { Brodaty et al., } \\
2005\end{array}$ & $\begin{array}{l}\text { "Friend or family } \\
\text { member offered } \\
\text { suggestions to } \\
\text { you to help with } \\
\text { caregiving", }\end{array}$ & $\begin{array}{l}\text { \# questions } \\
\text { for } \\
\text { respondent } \\
\text { burden. }\end{array}$ \\
\hline
\end{tabular}

CG-Caregiver, CR-Care Recipient a.k.a. Person with Dementia (PWD), N/A- Not Applicable, dx-diagnosis, Ed.- Education, $\boldsymbol{\alpha}$ Cronbach's alpha, e.g.-for example, r/t-related to; CRN:BR-Caregiver Research Network: Benjamin Rose; CRS - Carer Respite Survey; UNS - Unmet Needs Scale; SPS - Social Provision Scale; PGC-MAI - Philadelphia Geriatric Center Short-Length MultiLevel Assessment Instrument; $\boldsymbol{C A M}$ - Caregiver Appraisal Measure; ZBI - Zarit Burden Interview; CES-D - the Center for Epidemiologic Studies Depression Scale; JHDCNA - John's Hopkins Dementia Care Needs Assessment; REACH - Resources for Enhancing Alzheimer's Caregiver Health (REACH I 9/15/1995 through 8/31/2000); VCP - Victorian Carers Program; CPRSSCarer's Perceptions of Respite Services (O'Connell, Hawkins, Ostaszkiewicz, \& Millar, 2012) measures CG satisfaction and perceptions regarding respite care, part of the Carer Respite Survey (O'Connell et al., 2012). 
Appendix F Table 4. Expanded SPS and supporting literature

\begin{tabular}{|c|c|c|c|}
\hline Expanded SPS Question & $\begin{array}{l}\text { Reference } \\
\text { \& Method }\end{array}$ & Comment & $\begin{array}{c}\text { Andersen's } \\
\text { Model } \\
\text { Variable }\end{array}$ \\
\hline $\begin{array}{l}\text { Does the person with dementia see } \\
\text { his or her health care provider } \\
\text { regularly (for example: every } 6 \\
\text { months)? Response is yes/no and } \\
\text { comment by respondent. }\end{array}$ & $\begin{array}{l}\text { Phillipson } \\
\text { et al. (2014) } \\
\text { Quantitative }\end{array}$ & $\begin{array}{l}\text { Determines if physician } \\
\text { services were available, } \\
\text { which helps support the } \\
\text { CG role. }\end{array}$ & $\begin{array}{l}\text { Need, } \\
\text { Evaluated }\end{array}$ \\
\hline $\begin{array}{l}\text { Do you feel this frequency of } \\
\text { provider visits is adequate to meet } \\
\text { his or her (PWD) health care } \\
\text { needs? Response is yes/no and } \\
\text { comment by respondent. }\end{array}$ & $\begin{array}{l}\text { Phillipson } \\
\text { et al. (2014) } \\
\text { Quantitative }\end{array}$ & $\begin{array}{l}\text { Is provider evaluating } \\
\text { patient and does the CG } \\
\text { feel this is an } \\
\text { appropriate interval } \\
\text { (needs being met)? }\end{array}$ & $\begin{array}{l}\text { Need, } \\
\text { Perceived }\end{array}$ \\
\hline $\begin{array}{l}\text { Where did/do you obtain } \\
\text { information about dementia and } \\
\text { caregiving? (For example: the } \\
\text { health care provider's office, } \\
\text { family members, online websites } \\
\text { such as Alzheimers.org or the } \\
\text { Family Caregiving Alliance, or } \\
\text { other places). Response is } \\
\text { comment by respondent. }\end{array}$ & $\begin{array}{l}\text { Johnston et } \\
\text { al. (2011) } \\
\text { Quantitative }\end{array}$ & $\begin{array}{l}\text { Top three unmet needs } \\
\text { were education about } \\
\text { dementia; information } \\
\text { about resources and } \\
\text { services; \& CG mental } \\
\text { health } \\
\text { If the CG was unaware } \\
\text { of the available service, }\end{array}$ & $\begin{array}{l}\text { Need, } \\
\text { Perceived }\end{array}$ \\
\hline
\end{tabular}




\begin{tabular}{|c|c|c|c|}
\hline & $\begin{array}{l}\text { Ploeg et al. } \\
\text { (2009) } \\
\text { Quantitative } \\
\text { Stockwell- } \\
\text { Smith et al. } \\
\text { (2010) } \\
\text { Qualitative }\end{array}$ & $\begin{array}{l}\text { he or she did not use the } \\
\text { service or resource } \\
\text { CG and potential CG } \\
\text { identified the physician } \\
\text { as first choice to get } \\
\text { information, second } \\
\text { source was informal } \\
\text { support, and third choice } \\
\text { was home health } \\
\text { services } \\
\text { Findings indicated many } \\
\text { CG reported incorrect } \\
\text { information or lack of } \\
\text { knowledge about } \\
\text { available services }\end{array}$ & $\begin{array}{l}\text { Enabling } \\
\text { Organizing } \\
\text { (seeking } \\
\text { information } \\
\text { about } \\
\text { dementia) } \\
\text { Enabling, } \\
\text { Organizing }\end{array}$ \\
\hline $\begin{array}{l}\text { Did the health care provider refer } \\
\text { you to formal (paid) services for } \\
\text { caregiving? (Ex: home health, } \\
\text { Meals on Wheels, Adult Day Care, }\end{array}$ & $\begin{array}{l}\text { Robinson et } \\
\text { al. (2012) } \\
\text { Qualitative }\end{array}$ & $\begin{array}{l}\text { Identified formal and } \\
\text { informal services. } \\
\text { Getting correct } \\
\text { information to access }\end{array}$ & $\begin{array}{l}\text { Need, } \\
\text { Evaluated }\end{array}$ \\
\hline
\end{tabular}




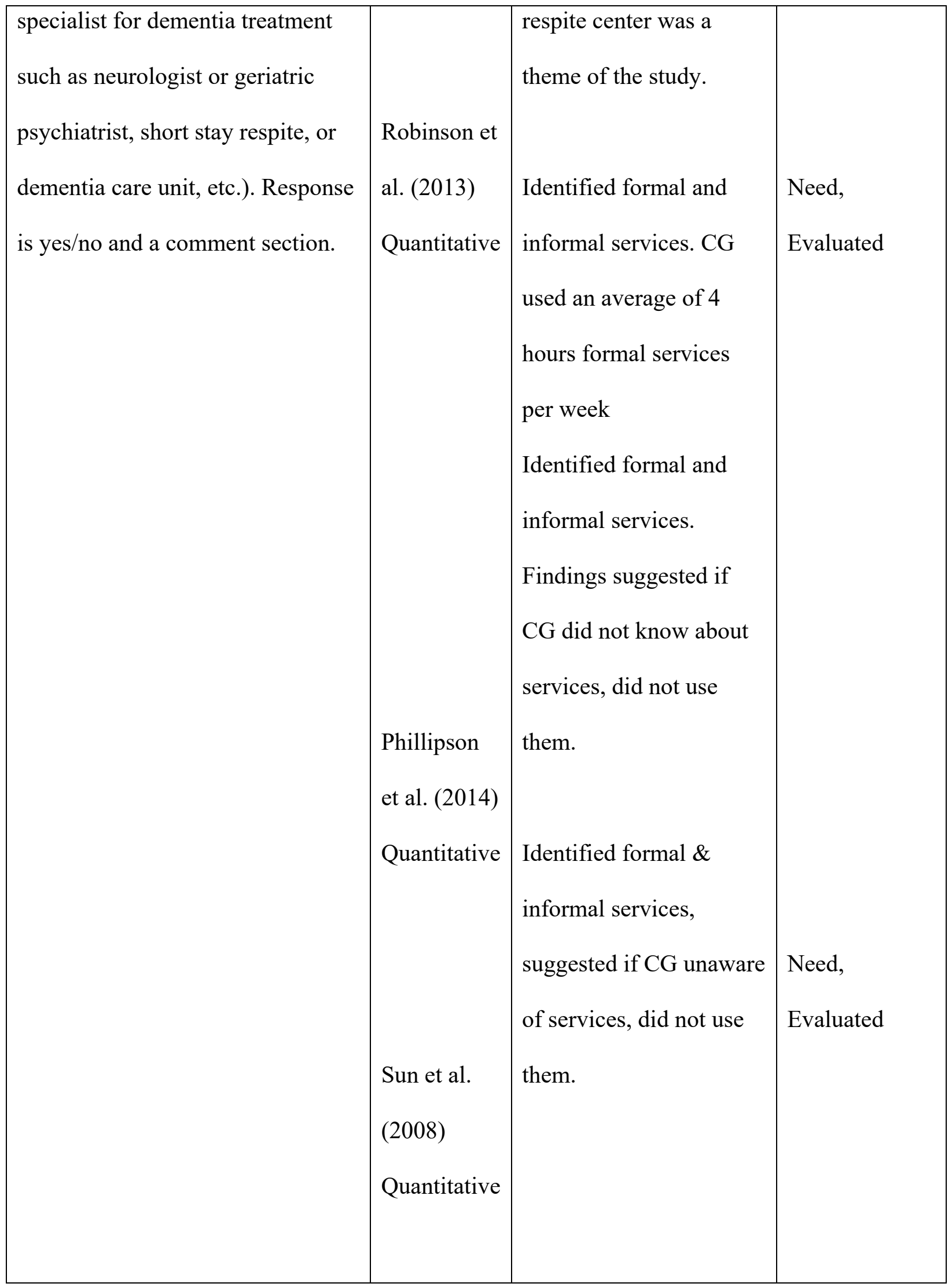




\begin{tabular}{|c|c|c|c|}
\hline & & $\begin{array}{l}\text { Identified specific } \\
\text { formal and informal } \\
\text { services. }\end{array}$ & $\begin{array}{l}\text { Need, } \\
\text { Evaluated }\end{array}$ \\
\hline $\begin{array}{l}\text { Would there be a formal service } \\
\text { you and the person with dementia } \\
\text { would use or be interested in } \\
\text { using? } \\
\text { Response is yes/no and comment. }\end{array}$ & $\begin{array}{l}\text { Phillipson } \\
\text { \& Jones } \\
\text { (2011; } \\
\text { 2012) } \\
\text { Qualitative } \\
\text { Phillipson } \\
\text { et al. (2013) } \\
\text { Quantitative }\end{array}$ & $\begin{array}{l}\text { If CG believed there } \\
\text { would be negative } \\
\text { outcome for CR, did not } \\
\text { use services, and if CG } \\
\text { belief was positive } \\
\text { outcome, tended to use } \\
\text { service. } \\
\text { Negative service beliefs } \\
\text { were more strongly } \\
\text { resource. } \\
\text { associated with non-use } \\
\text { of services than health } \\
\text { beliefs. }\end{array}$ & $\begin{array}{l}\text { Beliefs Service } \\
\text { Predisposing } \\
\text { Ber } \\
\text { Perceived } \\
\text { Need, }\end{array}$ \\
\hline
\end{tabular}




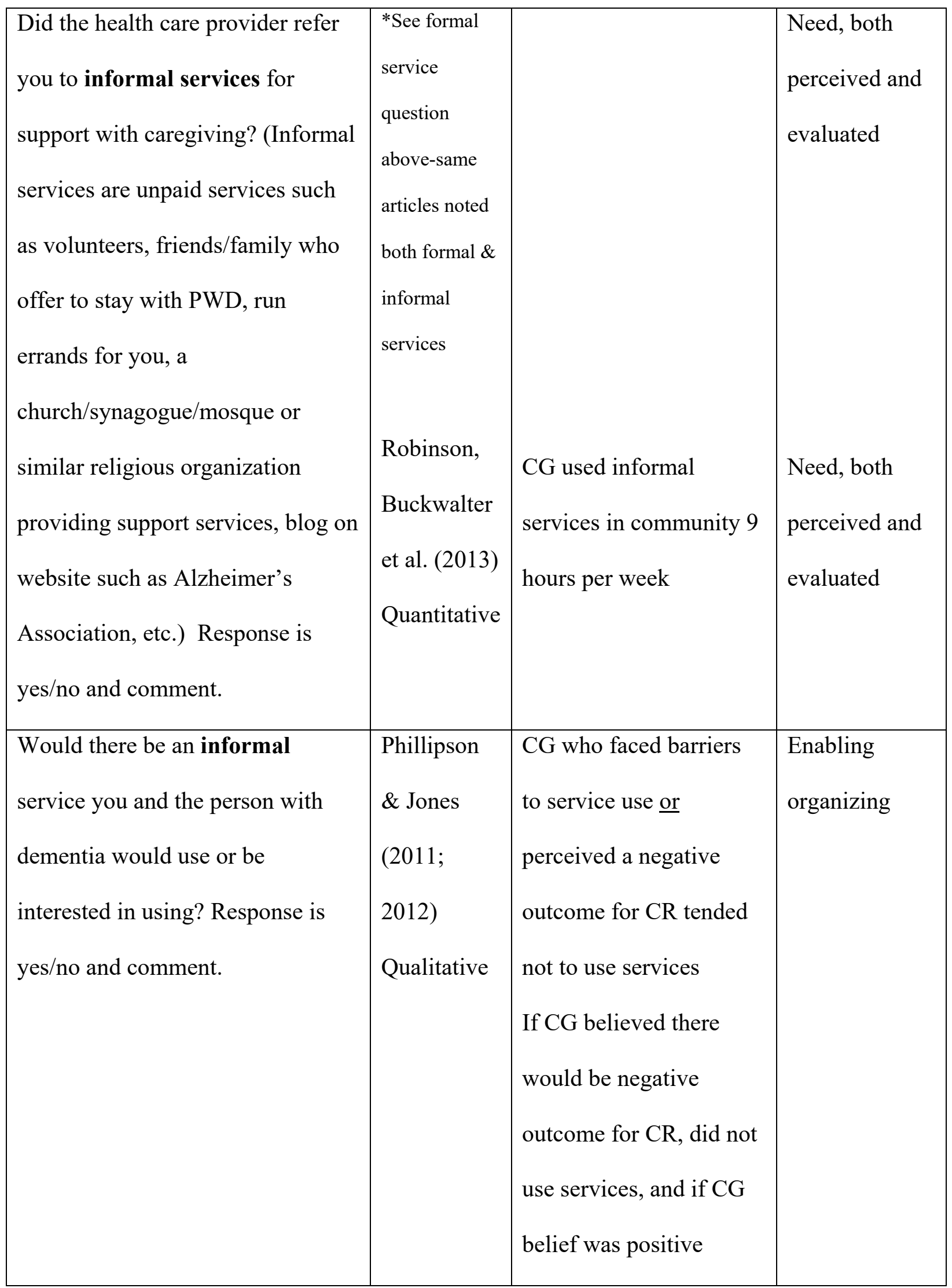




\begin{tabular}{|c|c|c|c|}
\hline & $\begin{array}{l} \\
\text { Phillipson } \\
\text { et al. (2013) } \\
\text { Quantitative }\end{array}$ & $\begin{array}{l}\text { outcome, tended to use } \\
\text { service. } \\
\text { Negative service beliefs } \\
\text { were more strongly } \\
\text { associated with non-use } \\
\text { of services than health } \\
\text { beliefs. }\end{array}$ & Predisposing \\
\hline $\begin{array}{l}\text { What is the health status of the } \\
\text { person with dementia? (Does he or } \\
\text { she have other chronic illnesses } \\
\text { such as high blood pressure, } \\
\text { diabetes, kidney disease, heart } \\
\text { failure, lung disease, etc.?) } \\
\text { Response is please explained. }\end{array}$ & $\begin{array}{l}\text { Brown \& } \\
\text { Chen, } \\
\text { (2008) } \\
\text { Qualitative } \\
\text { Phillipson } \\
\text { \& Jones } \\
\text { (2011; } \\
\text { 2012) } \\
\text { Qualitative }\end{array}$ & $\begin{array}{l}\text { CR change in behavior } \\
\text { or health precipitated } \\
\text { help-seeking } \\
\text { CG not using services } \\
\text { did not perceived current } \\
\text { need for respite but } \\
\text { reported may use in } \\
\text { future with deterioration } \\
\text { of CR functional and } \\
\text { cognitive state. A second } \\
\text { reason CG reported non- } \\
\text { use related to believing } \\
\text { the CR would have a } \\
\text { negative outcome. A }\end{array}$ & $\begin{array}{l}\text { Need, } \\
\text { Perceived }\end{array}$ \\
\hline
\end{tabular}




\begin{tabular}{|c|c|c|c|}
\hline & $\begin{array}{l}\text { Robinson et } \\
\text { al. (2005) } \\
\text { Quantitative }\end{array}$ & $\begin{array}{l}\text { third non-use reported } \\
\text { CG belief was that the } \\
\text { services could not } \\
\text { provide quality care for } \\
\text { the CR health or } \\
\text { behavior needs. } \\
\text { CG strongest reason to } \\
\text { use services: frequency } \\
\text { of CR ADL problems, } \\
\text { and CR frequency of } \\
\text { memory or behavior } \\
\text { problems (the strongest } \\
\text { reason to seek services) }\end{array}$ & $\begin{array}{l}\text { Need, } \\
\text { Perceived }\end{array}$ \\
\hline $\begin{array}{l}\text { What is the functional status of the } \\
\text { person with dementia? (Can CR } \\
\text { bathe, groom, and feed himself or } \\
\text { herself? Does CR manage own } \\
\text { finances? Does CR drive? Does } \\
\text { CR manage own medication } \\
\text { without help?) Response is } \\
\text { comment. }\end{array}$ & $\begin{array}{l}\text { *See above } \\
\text { for CR } \\
\text { health } \\
\text { status-same } \\
\text { references } \\
\& \\
\text { comments }\end{array}$ & $\begin{array}{l}\text { *See above for CR } \\
\text { health status-same } \\
\text { references and } \\
\text { comments }\end{array}$ & $\begin{array}{l}\text { Need, } \\
\text { Perceived }\end{array}$ \\
\hline
\end{tabular}




\begin{tabular}{|c|c|c|c|}
\hline $\begin{array}{l}\text { How would you describe your } \\
\text { health status? (Do you have other } \\
\text { chronic illnesses such as high } \\
\text { blood pressure, diabetes, kidney } \\
\text { disease, heart failure, lung disease, } \\
\text { etc.?) Response is comment. }\end{array}$ & $\begin{array}{l}\text { O’Connell } \\
\text { et al.(2012) } \\
\text { Quantitative }\end{array}$ & $\begin{array}{l}\text { Reported main reasons } \\
\text { for use of respite were } \\
\text { CG own health and } \\
\text { needs }\end{array}$ & $\begin{array}{l}\text { Need, } \\
\text { Perceived }\end{array}$ \\
\hline $\begin{array}{l}\text { How would you describe your own } \\
\text { functional status? (Are you able to } \\
\text { care for yourself, walk without } \\
\text { assistance, drive, etc.?). Response } \\
\text { is comment. }\end{array}$ & $\begin{array}{l}\text { O'Connell } \\
\text { et al. (2012) } \\
\text { Quantitative }\end{array}$ & $\begin{array}{l}\text { Reported main reasons } \\
\text { for use of respite were } \\
\text { CG own health and } \\
\text { needs }\end{array}$ & $\begin{array}{l}\text { Need, } \\
\text { Perceived }\end{array}$ \\
\hline $\begin{array}{l}\text { Do you live with the person with } \\
\text { dementia (in same residence such } \\
\text { as apartment, house, trailer, etc.)? } \\
\text { Response is yes/no. }\end{array}$ & $\begin{array}{l}\text { Robinson et } \\
\text { al. (2013) } \\
\text { Quantitative }\end{array}$ & $\begin{array}{l}\text { Service non-users lived } \\
\text { with CR }\end{array}$ & $\begin{array}{l}\text { Enabling, } \\
\text { Organizing }\end{array}$ \\
\hline $\begin{array}{l}\text { Are you working at another job or } \\
\text { place of employment in addition to } \\
\text { the caregiving role? Response is } \\
\text { yes/no. }\end{array}$ & $\begin{array}{l}\text { O’Connell } \\
\text { et al. (2012) } \\
\text { Quantitative }\end{array}$ & $\begin{array}{l}\text { More use of services if } \\
\text { accommodated CG work } \\
\text { schedule. Also, fourth } \\
\text { reason to use respite } \\
\text { services for CR was to } \\
\text { meet CG work } \\
\text { commitments (overnight } \\
\text { respite most used service }\end{array}$ & $\begin{array}{l}\text { Enabling, } \\
\text { Organizing } \\
\text { (* possible } \\
\text { Financial also) }\end{array}$ \\
\hline
\end{tabular}




\begin{tabular}{|c|c|c|c|}
\hline & & $\begin{array}{l}\text { if CG had health issues } \\
\text { or work issues) }\end{array}$ & \\
\hline $\begin{array}{l}\text { If yes (you are working another } \\
\text { job), how many hours per week do } \\
\text { you work at the job? Response is } \\
\text { to choose one of the following: } \\
\text { between } 1-10,11-20,21-30,31-40 \text {, } \\
\text { or more than } 41 \text { hours per week. }\end{array}$ & $\begin{array}{l}\text { *Added to } \\
\text { Quantify \# } \\
\text { hours } \\
\text { worked. }\end{array}$ & $\begin{array}{l}\text { Benjamin Rose Institute: } \\
\text { CGN suggested question }\end{array}$ & $\begin{array}{l}\text { Enabling, } \\
\text { Organizing } \\
(* \text { possible } \\
\text { Financial also) }\end{array}$ \\
\hline $\begin{array}{l}\text { Does the person with dementia } \\
\text { have health insurance to help with } \\
\text { medical needs (such as } \\
\text { prescription medications, } \\
\text { wheelchair, walker, etc.)? } \\
\text { Response is yes/no and comment. }\end{array}$ & $\begin{array}{l}\text { Phillipson } \\
\text { et al. (2014: } \\
\text { 2013) } \\
\text { Quantitative }\end{array}$ & $\begin{array}{l}\text { Health insurance assists } \\
\text { with affording potential } \\
\text { services. }\end{array}$ & $\begin{array}{l}\text { Enabling, } \\
\text { Financial }\end{array}$ \\
\hline CG age and level of education & $\begin{array}{l}\text { Phillipson } \\
\text { et al. (2014; } \\
\text { 2013) } \\
\text { Quantitative } \\
\text { O'Connell } \\
\text { et al. (2012) } \\
\text { Quantitative }\end{array}$ & $\begin{array}{l}\text { CG who were older } \\
\text { tended not to use } \\
\text { services. } \\
\text { Assessed CG age and } \\
\text { level of education }\end{array}$ & $\begin{array}{l}\text { Predisposing } \\
\text { demographic } \\
\text { Predisposing } \\
\text { demographic } \\
\text { age; } \\
\text { Predisposing }\end{array}$ \\
\hline
\end{tabular}




\begin{tabular}{|c|c|c|c|}
\hline & $\begin{array}{l}\text { Robinson et } \\
\text { al. (2013) } \\
\text { Quantitative }\end{array}$ & $\begin{array}{l}\text { Non-service user CGs } \\
\text { were older, lived with } \\
\text { CR, and were spouses. } \\
\text { Additionally, CG } \\
\text { received less social } \\
\text { support and reported } \\
\text { more depressive } \\
\text { symptoms but provided } \\
\text { fewer hours of care to } \\
\text { CR with fewer } \\
\text { cognitive, functional, } \\
\text { and behavior problems. } \\
\text { physician as main source } \\
\text { of support }\end{array}$ & $\begin{array}{l}\text { Social level of } \\
\text { education } \\
\text { Non-Users } \\
\text { (outcome) } \\
\text { Social } \\
\text { Predisposing } \\
\text { Soln }\end{array}$ \\
\hline $\begin{array}{l}\text { Is anyone helping you with } \\
\text { caregiving? }\end{array}$ & $\begin{array}{l}\text { Phillipson } \\
\text { et al. (2013) }\end{array}$ & $\begin{array}{l}\text { Informal support for } \mathrm{CG} \\
\text { in caring role }\end{array}$ & $\begin{array}{l}\text { Enabling, } \\
\text { Organizing }\end{array}$ \\
\hline
\end{tabular}

CG-Caregiver, CR-Care Recipient a.k.a. Person with Dementia (PWD) 
Appendix F Social Provisions Scale-Short Version

Social Provisions Scale-Short Version

Copyright by Daniel Russell and Carolyn Cutrona, 1984

$\begin{array}{cccc}\text { STRONGLY DISAGREE } & \text { DISAGREE } & \text { AGREE } & \text { STRONGLY AGREE } \\ 1 & 2 & 3 & 4\end{array}$

$\underline{\text { Rating }}$

1. There are people I can depend on to help me if I really need it.

2. I feel that I do not have close personal relationships with other people.

3. There is no one I can turn to for guidance in times of stress.

4. There are people who enjoy the same social activities that I do.

5. I do not think other people respect my skills and abilities.

6. If something went wrong, no one would come to my assistance.

7. I have close relationships that provide me with a sense of emotional security and well-being.

8. I have relationships where my competence and skills are recognized.

9. There is no one who shares my interests and concerns.

10. There is a trustworthy person I could turn to for advice if I were having problems.

Scoring: 
A score for each social provision is derived such that a high score indicates that the individual is receiving that provision. Items that are asterisked should be reversed before scoring (i.e., $4=1$, $3=2,2=3,1=4)$.

1. Guidance: $3^{*}, 16$

2. Reassurance of Worth: $9 *, 13$

3. Social Integration: $5,14^{*}$

4. Attachment: 2*,11

5. Reliable Alliance: $1,10^{*}$ 
Appendix F Social Provisions Scale 24 item

\section{Social Provisions Scale}

\section{(C) Daniel Russell \& Carolyn Cutrona, 1984}

Instructions: In answering the following questions, think about your current relationships with friends, family members, co-workers, community members, and so on. Please indicate to what extent each statement describes your current relationships with other people. Use the following scale to indicate your opinion.

\section{STRONGLY DISAGREE DISAGREE 1

So, for example, if you feel a statement is very true of your current relationships, you would respond with a 4 (strongly agree). If you feel a statement clearly does not describe your relationships, you would respond with a 1 (strongly disagree).

$\underline{\text { Rating }}$

1. There are people I can depend on to help me if I really need it.

2. I feel that I do not have close personal relationships with other people.

3. There is no one I can turn to for guidance in times of stress.

4. There are people who depend on me for help.

5. There are people who enjoy the same social activities I do.

6. Other people do not view me as competent.

7. I feel personally responsible for the well-being of another person.

8. I feel part of a group of people who share my attitudes and beliefs.

9. I do not think other people respect my skills and abilities. 
10. If something went wrong, no one would come to my assistance.

11. I have close relationships that provide me with a sense of emotional security and well-being.

12. There is someone I could talk to about important decisions in my life.

13. I have relationships where my competence and skill are recognized.

14. There is no one who shares my interests and concerns.

Rating

15. There is no one who really relies on me for their well-being.

16. There is a trustworthy person I could turn to for advice if I were having problems.

17. I feel a strong emotional bond with at least one other person.

18. There is no one I can depend on for aid if I really need it.

19. There is no one I feel comfortable talking about problems with.

20. There are people who admire my talents and abilities.

21. I lack a feeling of intimacy with another person.

22. There is no one who likes to do the things I do.

23. There are people who I can count on in an emergency.

24. No one needs me to care for them.

Scoring:

A score for each social provision is derived such that a high score indicates that the individual is receiving that provision. Items that are asterisked should be reversed before scoring (i.e., $4=1,3=2,2=3,1=4$ ). 
1. Guidance: $3^{*}, 12,16,19^{*}$

2. Reassurance of Worth: $6^{*}, 9^{*}, 13,20$

3. Social Integration: $5,8,14^{*}, 22^{*}$

4. Attachment: $2^{*}, 11,17,21^{*}$

5. Nurturance: $4,7,15^{*}, 24^{*}$

6. Reliable Alliance: $1,10^{*}, 18^{*}, 23$ 
Appendix F Expanded Social Provisions Scale

\section{Expanding the SPS 24-item Trial Additional Questions}

Please complete this AFTER you completed the SPS 24-item.

Does the person with dementia see his or her health care provider regularly? (For example: every 6 months) Yes No

Comments:

Do you feel the number of health care visits is adequate to meet the person with dementia's health care needs? Yes No

Comments:

Where did/do you obtain information about dementia and caregiving? (For example: the health care provider's office, family members, online websites such as Alzheimer's.org or the Family Caregiver Alliance, or other places)

Did the health care provider refer you to formal services for support with caregiving? (Formal services are those services that cost money, such as Home Health services, Meals on Wheels or other meal services, Adult Day Care Services, a specialist for dementia such as a geriatric psychiatrist or neurologist, short stay respite in a nursing home or dementia care unit, etc.)<smiles></smiles>

Comment: 
Would there be a formal service you and the person with dementia would use or be interested in using? _ Yes _ _ No

Please explain:

Did the health care provider refer you to informal services for support with caregiving?

(Informal services are unpaid services, such as volunteers, friends or family who offer to stay with the person with dementia, run errands for you, a church/synagogue/mosque or similar religious organization providing support services, the Alzheimer's Association website or similar website, etc.). __ Yes _ No Comment:

Would there be an informal service you and the person with dementia would use or be interested in using? _ Yes _ N No Please explain:

What is the health status of the person with dementia? (Does he or she have other chronic illnesses such as high blood pressure, diabetes, kidney disease, heart failure, lung disease such as asthma or chronic obstructive pulmonary disease [COPD], etc.?)

What is the functional status of the person with dementia? (Can he or she bathe, groom, feed himself or herself? Does he or she manage his or her own finances? Does he or she drive? Does the person with dementia manage his or her own medications without help?) Comment: 
What is your age? Years

What is the highest level of education you have completed?

$\square$ Elementary

$\square$ Middle School/Junior High

High School/GED

$\square$ Some College

$\square \quad$ Trade School

$\square$ Bachelor's Degree

$\square$ Some Graduate School

$\square$ Graduate Degree

$\square \quad$ Advanced Graduate Studies/PhD

$\square$ Do not know/unsure 
Does anyone assist you with caregiving for the person with dementia? _ Y Yes _ _ No How do they assist you? (listening to you, providing transportation, staying with the person with dementia while you run errands, sharing the care of the person with dementia, bringing you meals, etc.?

How would you describe your health status? (Do you have other chronic illnesses such as high blood pressure, diabetes, kidney disease, heart failure, lung disease such as asthma or chronic obstructive pulmonary disease [COPD], etc.?)

Comment:

How would you describe your own functional status? (Are you able to care for yourself, walk without assistance, drive, etc.?)

Comment:

Do you live with the person with dementia (in the same residence such as apartment, house, trailer, etc.) ? _ Y Yes _ No

Are you working at another job or place of employment in addition to the caregiving role? Yes _ No

If yes, how many hours per week do you work at that job? 1-10 hours per week 11-20 hours per week 21-30 hours per week 
31-40 hours per week

More than 41 hours per week

Does the person with dementia have health insurance to help with medical needs (such as prescription medications, wheelchairs, walkers, etc.)? Yes No

Comment: 


\section{Appendix G Permission Letters for Measures}

The researcher, Kristina M. Childers, requested permission from authors of each scale. The following are emails from the authors, or the link for those in public domain.

\section{Unmet Needs Assessment}

\section{Dr. Bass Permission Letter for Unmet Needs Assessment}

Dear Ms. Childers,

You have Dr. Bass's permission to use the Benjamin Rose Institute Unmet Need Scale (attached) for the purposes you outlined in your email. Please use the following reference when citing the tool:

Bass, D.M., Judge, K.S., Snow, L., Wilson, N., Looman, W., McCarthy, K., Morgan, R., \& C. Abloorh-

Odjidja, C., Kunik, M. (2012). Negative caregiving effects among caregivers of veterans with dementia. The American Journal of Geriatric Psychiatry; 20: 239-247. doi: 10.1097/JGP.0b013e31824108ca.

We'd be interested in hearing how your dissertation goes and whether the tool ends up being useful.

Regards,

Kate McCarthy

Senior Research Assistant 
Appendix G Permission Letter Philadelphia Geriatric Center Multilevel Assessment Instrument (PCG-MAI)

Lawton, Moss, Fulcomer, and Kleban (1982)

The instrument is Public Domain and may be used if proper attribution is given.

Permission is Granted for the Philadelphia Geriatric Center Multilevel Assessment Instrument

(PGC-MAI) https://www.abramsoncenter.org/media/1201/lawtons-pgc-multi-level-assessmentinstrument.pdf

The above link will take you to a 135-page pdf document. Page 2 is where permission is granted.

This scale is in the public domain and may be reproduced with proper attribution.

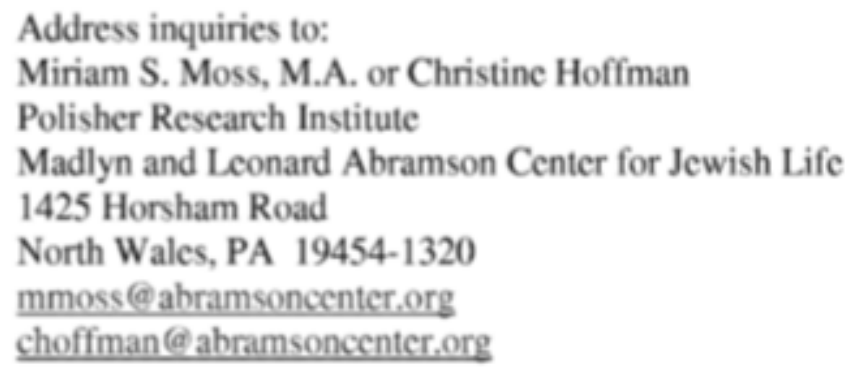


Appendix G Permission Letter Caregiver Mastery Subscale, part of the Caregiving Appraisal

Scale

Lawton, Moss, Hoffman, and Perkinson (2000, p.2).

Permission is granted to use or reproduce this scale, provided that proper attribution is given.

The permission for the Caregiver Mastery Subscale is below and also available from

https://www.abramsoncenter.org/media/1194/caregiving-appraisal-scale.pdf 


\section{Appendix G Permission Letter Dr. Lyn Phillipson}

Permission from Dr. Lyn Phillipson for use of questions about Health (Dementia) Beliefs and Beliefs about Services (Dementia)

My PhD thesis is available for free download from the University of Wollongong Library at: http://ro.uow.edu.au/theses/

All of the research instruments, information and consent forms are available for your reference in the Appendices of the thesis. This should assist you to answer your questions below regarding the scales utilised etc in the different survey questions.

All the best with the thesis. I would be interested to know about your results. The study of particular cultural groups and their service utilisation is a very interesting and important one so good luck!

As acknowledgement of the use of my work, can I please ask that you reference the use of the survey and any scales as:

Phillipson, L., Magee, C., \& Jones, S. C. (2013). Why carers of people with dementia do not utilise out-of-home respite services. Health \& Social Care in the Community, 21(4), 411-422. doi: $10.1111 /$ hsc. 12030

Please let me know if I can be of any further assistance

Sincerely

Lyn

Dr Lyn Phillipson

Senior Lecturer, School of Health and Society Associate Director, Centre for Health Initiatives

Faculty of Social Sciences

University of Wollongong

$+61242214773$

Iphillip@uow.edu.au 


\section{Appendix G Permission Dr. Gitlin}

Gitlin questions from Resources for Enhancing Caregiver Health (R.E.A.C.H.) are discussed in Gitlin, Roth, and Huang (2014). The variables and questions are available

@ https://www.icpsr.umich.edu/icpsrweb/ICPSR/studies/03678/datasets/0014/variables/HOMH? $\underline{\operatorname{archive}=\mathrm{ICPSR}}$.

A rationale for scoring is included in Gitlin, Roth, and Huang (2014)

(a) https://www.ncbi.nlm.nih.gov/pmc/articles/PMC4176772/: “A total utilization index was computed by summing the number of services used".

The R.E.A.C.H. data set is available from the Inter-University Consortium for Political and Social Research (ICPSR) (Shulz, 2006)

@ https://www.icpsr.umich.edu/icpsrweb/ICPSR/studies/03678.

Additional documentation on the Instrument "Formal Care and Services Use (REACH II)" is found on page 23 of Evaluating Dementia Services Evaluating Dementia Services and Supports: Instrument Resource List as prepared for E. Long@ @ https://nadrc.acl.gov/node/70 “Credit the developers by using the complete citation. No other permissions are required."

The public-use data files in this collection are available for access by the general public. Access does not require affiliation with an ICPSR member institution. 


\section{Notes}

- The public-use data files in this collection are available for access by the general public. Access does not require affiliation with an ICPSR member institution.

- The citation of this study may have changed due to the new version control system that has been implemented. Please see version history for more details.

\section{NACDA \\ Program on Aging}

This study is maintained and distributed by the National Archive of Computerized D.ata on Aging (NACDA), the aging program within ICPSR. NACDA is sponsored by the National Institute on Aging (NIA) at the National Institutes of Heath $(\mathrm{NIH})$. 
Appendix G Letter of Permission Social Provisions Scale (SPS) Russell and Cutrona 2015

to me

\section{Russell, Daniel W [HD FS] <drussell@iastate.edu>}

Aug 3, 2015,

4:01 PM

You have my permission to use the Social Provisions Scale as part of your dissertation research.

Daniel W. Russell, Ph.D.

Professor, Department of Human

Development \& Family Studies

lowa State University

(515) 294-4187

Fax: 294-2502

Dr. Cutrona provided permission and attached the scales and information to the email.

Cutrona, Carolyn [PSYCH] <ccutrona@iastate.edu>

to me $\mathrm{v}$

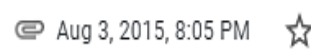

You have my permission to use the Social Provisions Scale. Are you referring to the 10-item version?

All the best,

Carolyn Cutrona

From: Kristi Childers [mailto:kristikylechilders@gmail.com]

Sent: Monday, August 03, 2015 12:51 PM

To: bgottlie@uoguelph.ca; Russell, Daniel W [HD FS] 〈drussell@iastate.edu〉; Cutrona, Carolyn [PSYCH] <ccutrona@iastate.edu>

Subject: SPS-10

*..

3 Attachments
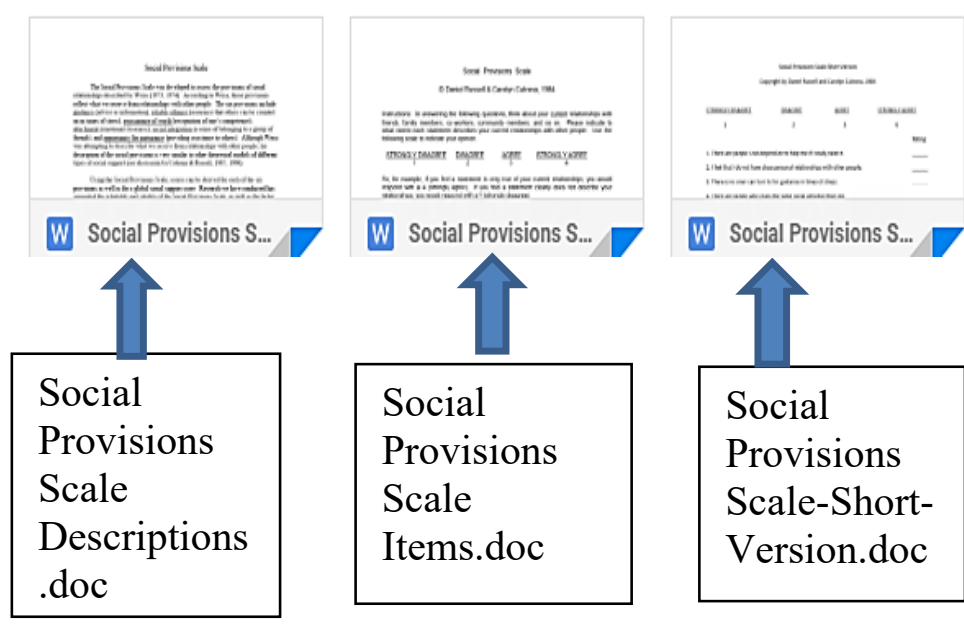

Social

Provisions

Scale

Items.doc

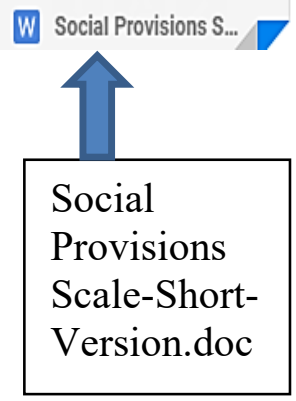


Appendix H Expanded Social Provisions Scale

\section{Expanding the SPS 24-item Trial Additional Questions}

Please complete this AFTER you completed the SPS 24-item.

Does the person with dementia see his or her health care provider regularly? (For example: every 6 months) Yes No

Comments:

Do you feel the number of health care visits is adequate to meet the person with dementia's health care needs? Yes No

Comments:

Where did/do you obtain information about dementia and caregiving? (For example: the health care provider's office, family members, online websites such as Alzheimer's.org or the Family Caregiver Alliance, or other places)

Did the health care provider refer you to formal services for support with caregiving?

(Formal services are those services that cost money, such as Home Health services, Meals on Wheels or other meal services, Adult Day Care Services, a specialist for dementia such as a geriatric psychiatrist or neurologist, short stay respite in a nursing home or dementia care unit, etc.)

Yes No

Comment: 
Would there be a formal service you and the person with dementia would use or be interested in using? __ Yes __ No

Please explain:

Did the health care provider refer you to informal services for support with caregiving?

(Informal services are unpaid services, such as volunteers, friends or family who offer to stay with the person with dementia, run errands for you, a church/synagogue/mosque or similar religious organization providing support services, the Alzheimer's Association website or similar website, etc.). ___ Yes _ No Comment:

Would there be an informal service you and the person with dementia would use or be interested in using? _ Please explain:

What is the health status of the person with dementia? (Does he or she have other chronic illnesses such as high blood pressure, diabetes, kidney disease, heart failure, lung disease such as asthma or chronic obstructive pulmonary disease [COPD], etc.?)

What is the functional status of the person with dementia? (Can he or she bathe, groom, feed himself or herself? Does he or she manage his or her own finances? Does he or she drive? Does the person with dementia manage his or her own medications without help?)

Comment: 
What is your age? Years

What is the highest level of education you have completed?

$\square \quad$ Elementary

$\square$ Middle School/Junior High

$\square$ High School/GED

$\square$ Some College

$\square \quad$ Trade School

$\square$ Bachelor's Degree

$\square \quad$ Some Graduate School

$\square$ Graduate Degree

$\square \quad$ Advanced Graduate Studies/PhD

$\square$ Do not know/unsure 
Does anyone assist you with caregiving for the person with dementia? _ Yes _ _ No

How do they assist you? (listening to you, providing transportation, staying with the person with dementia while you run errands, sharing the care of the person with dementia, bringing you meals, etc.?

How would you describe your health status? (Do you have other chronic illnesses such as high blood pressure, diabetes, kidney disease, heart failure, lung disease such as asthma or chronic obstructive pulmonary disease [COPD], etc.?)

Comment:

How would you describe your own functional status? (Are you able to care for yourself, walk without assistance, drive, etc.?)

Comment:

Do you live with the person with dementia (in the same residence such as apartment, house, trailer, etc.) ? _ Y Yes _ No

Are you working at another job or place of employment in addition to the caregiving role? Yes No

If yes, how many hours per week do you work at that job?

1-10 hours per week 11-20 hours per week 21-30 hours per week 
31-40 hours per week

More than 41 hours per week

Does the person with dementia have health insurance to help with medical needs (such as prescription medications, wheelchairs, walkers, etc.)? Yes No

Comment: 
Appendix I Research Survey

SURVEY APPALACHIAN CAREGIVERS

Research ID: 1604083616

The following questions ask about you as the caregiver and the care recipient (person with dementia).

\begin{tabular}{|c|c|c|}
\hline Question & Caregiver & $\begin{array}{c}\text { Care Recipient (Person } \\
\text { with Dementia) }\end{array}$ \\
\hline What is the gender? & $\begin{array}{ll}\square & \text { Male } \\
\square & \text { Female }\end{array}$ & $\begin{array}{ll}\square & \text { Male } \\
\square & \text { Female }\end{array}$ \\
\hline What is age in years? & Years & Years \\
\hline What race is identified? & $\begin{array}{ll} & \text { American Indian or } \\
& \text { Alaskan Native } \\
\square & \text { Asian or Pacific } \\
\text { Island } \\
\square \quad \text { Black (not Hispanic) } \\
\square \quad \text { Hispanic or Latino } \\
\square \quad \text { White (not Hispanic) } \\
\square \quad \text { Other } \\
\end{array}$ & $\begin{array}{ll} & \text { American Indian or } \\
& \text { Alaskan Native } \\
\square & \text { Asian or Pacific } \\
& \text { Island } \\
\square & \text { Black (not Hispanic) } \\
\square & \text { Hispanic or Latino } \\
\square & \text { White (not Hispanic) } \\
\square & \text { Other } \\
\end{array}$ \\
\hline $\begin{array}{l}\text { What language is used } \\
\text { at home? }\end{array}$ & $\begin{array}{ll}\square & \text { English } \\
\square & \text { Spanish } \\
\square & \text { Chinese } \\
\square & \text { Other }\end{array}$ & $\begin{array}{ll}\square & \text { English } \\
\square & \text { Spanish } \\
\square & \text { Chinese } \\
\square & \text { Other }\end{array}$ \\
\hline Marital Status & $\begin{array}{ll}\square & \text { Single } \\
\square & \text { In a relationship } \\
\square & \text { Married } \\
\square & \text { Divorced/Separated } \\
\square & \text { Widow/widower }\end{array}$ & $\begin{array}{ll}\square & \text { Single } \\
\square & \text { In a relationship } \\
\square & \text { Married } \\
\square & \text { Divorced/Separated } \\
\square & \text { Widow/widower }\end{array}$ \\
\hline $\begin{array}{l}\text { Highest Level of } \\
\text { Education completed? }\end{array}$ & $\begin{array}{ll}\square & \text { Less than High School } \\
\square \quad \text { High School (or GED) } \\
\square \quad \text { Post High School } \\
\square \quad \text { College Degree } \\
\square \quad \text { Profession/Grad Degree } \\
\square \quad \text { Do not know/unsure }\end{array}$ & $\begin{array}{ll}\square & \text { Less than High School } \\
\square & \text { High School (or GED) } \\
\square & \text { Post High School } \\
\square & \text { College Degree } \\
\square & \text { Profession/Grad Degree } \\
\square & \text { Do not know/unsure }\end{array}$ \\
\hline
\end{tabular}




\begin{tabular}{|c|c|c|}
\hline Question & Caregiver & $\begin{array}{c}\text { Care Recipient (Person } \\
\text { with Dementia) }\end{array}$ \\
\hline $\begin{array}{l}\text { What is current } \\
\text { household income in } \\
\text { U.S. dollars? }\end{array}$ & $\begin{array}{ll}\square & \text { Under } \$ 10,000 \\
\square & \$ 10,000-\$ 39,999 \\
\square & \$ 40,000-\$ 79,999 \\
\square & \$ 80,000-\$ 124,999 \\
\square & \$ 125,000-\$ 150,000 \\
\square & \text { Over } \$ 150,000 \\
\square & \text { Would rather not say }\end{array}$ & $\begin{array}{ll}\square & \text { Under } \$ 10,000 \\
\square & \$ 10,000-\$ 39,999 \\
\square & \$ 40,000-\$ 79,999 \\
\square & \$ 80,000-\$ 124,999 \\
\square & \$ 125,000-\$ 150,000 \\
\square & \text { Over } \$ 150,000 \\
\square & \text { Would rather not say }\end{array}$ \\
\hline $\begin{array}{l}\text { Do you own your } \\
\text { home? }\end{array}$ & $\begin{array}{l}\square \text { Yes } \\
\square \text { No }\end{array}$ & $\begin{array}{l}\square \text { Yes } \\
\square \quad \text { No }\end{array}$ \\
\hline $\begin{array}{l}\text { Are there Advanced } \\
\text { Care Directives } \\
\text { (paperwork that tells your } \\
\text { doctors and /or family what } \\
\text { to do if you aren't capable) }\end{array}$ & $\begin{array}{l}\square \text { Yes } \\
\square \text { No }\end{array}$ & $\begin{array}{l}\square \quad \text { Yes } \\
\square \quad \text { No }\end{array}$ \\
\hline $\begin{array}{l}\text { Is there a primary } \\
\text { health care provider? } \\
\text { (Physician, Nurse } \\
\text { practitioner, PA?) }\end{array}$ & $\begin{array}{l}\square \text { Yes } \\
\square \text { No }\end{array}$ & $\begin{array}{l}\square \text { Yes } \\
\square \text { No }\end{array}$ \\
\hline $\begin{array}{l}\text { Are there chronic } \\
\text { health conditions } \\
\text { treated with } \\
\text { medications or other }\end{array}$ & $\begin{array}{l}\square \quad \text { Yes } \\
\square \text { No }\end{array}$ & $\begin{array}{l}\text { In addition to the } \\
\text { diagnosed Dementia } \\
\square \text { Yes } \\
\square \text { No }\end{array}$ \\
\hline
\end{tabular}




\begin{tabular}{|l|l|l|}
\hline \multicolumn{1}{|c|}{ Question } & Caregiver & Care Recipient (Person \\
with Dementia)
\end{tabular}

What is your relationship to the person for whom you provide care?

Spouse or Partner

Adult child

Sibling
Friend/other non-relative

Other:

Did your health care provider recommend services for the person with dementia?

Yes

No

What is the diagnosed type of dementia of the person for whom you care?

Alzheimer's Disease

Dementia

Dementia with Lewy bodies

Frontotemporal Dementia

Parkinson's Dementia

Huntington's Disease

Wernicke-Korsakoff Syndrome
Mixed Dementia (two or more types)

Vascular Dementia

Other:

I do not know or cannot remember. 
When was care recipient diagnosed with dementia? year(s) months ago

How long have you been providing care?
1 day to 6 months
5 to 7 years
7 months to 1 year
8 to 10 years
1 to 2 years
Over 10 years
3 to 4 years

How many HOURS PER WEEK do YOU provide care, assistance, supervision or companionship to the care recipient (person with dementia)

Less than 6 hours

6-10 hours

11-15 hours

16-20 hours

21-25 hours

26-30 hours

31-35 hours

36-40 hours

$\square$ Over 40 hours

Do you live in the same home as the care recipient?
Yes
No

\section{Where are you currently living?}

Private home/apartment with no home care services

Private home/apartment with home care services or supported housing services Boarding house or other care/group home Assisted Living

$\square$ Other 
Where is the care recipient currently living?

Private home/apartment with no home care services

Private home/apartment with home care services or supported housing

Boarding house or other care/group home

Assisted Living

Nursing home

Other

Who are you currently living with?

Live alone

Live with spouse only

Live with spouse and others

Live with child

Live with other(s) (not related)

Live in group setting with nonrelative(s) (e.g. nursing home, Intermediate care facility, group home, assisted living)

Live with parent(s)

In what state and county do you reside?

County

WV

VA

$\mathrm{OH}$

KY

PA

Other

In what state and county does the person for whom you provide care (person with dementia) reside?

County:

WV

VA

$\mathrm{OH}$

KY

PA

Other

Are you the main (primary) caregiver for the person with dementia?

Yes

No, I am one of two or more caregivers who share in care

How many other people do you provide unpaid care or assistance for in addition to the person with dementia?

None (0)

2

1

3 or more 
On average, how many HOURS PER WEEK of PAID help cares for the person with dementia? (Excluding residential care; including adult day care, home attendant care, etc.)

HOURS/WEEK

On average, how many HOURS PER WEEK of UNPAID help is received from family, friends, or volunteers other than you?

HOURS/WEEK

Are you employed outside the home?

No, I am retired

$\square$ No, I am a full-time

homemaker

No, I am disabled

$\square$ Yes, I work full time (36-40

hours per week)
Yes, I work part time (less than

36 hours, more than 12 hours)

Yes, I work part time (4- 12

hours per week)

Do you have faith (spiritual) beliefs that affect important decisions in your life?

$\square$ Yes

$\square$ No

Do you regularly attend church/synagogue/mosque or faith-based meetings?

Yes

$\square$ No 
On the Rating line next to each item put in a number from 1 STRONGLY DISAGREE to 4 STRONGLY AGREE.

$\underline{\text { STRONGLY DISAGREE DISAGREE }} \underline{\text { AGREE }}$ STRONGLY AGREE

$\begin{array}{llll}1 & 2 & 3 & 4\end{array}$

\section{$\underline{\text { Rating }}$}

1. There are people I can depend on to help me if I really need it.

2. I feel that I do not have close personal relationships with other people.

3. There is no one I can turn to for guidance in times of stress.

4. There are people who enjoy the same social activities that I do.

5. I do not think other people respect my skills and abilities.

6. If something went wrong, no one would come to my assistance.

7. I have close relationships that provide me with a sense of emotional security and well-being.

8. I have relationships where my competence and skills are recognized.

9. There is no one who shares my interests and concerns.

10. There is a trustworthy person I could turn to for advice if I were having problems. 
Please read the list of topics. After reading each topic, I would like you to tell me if you or the person with dementia needs more information or help with this topic. Circle No (0) or Yes (1).

\section{Do you need more information about or help with this?}

a) Getting family members or friends to help your loved one with dementia because of his/her memory problems?

b) How to discuss your loved one with dementia's memory problems with other family members?

c) Planning who would help your loved one with dementia if you were no longer able to help?

d) Discussing the future course of your loved one with dementia's illness with other family members?

e) Getting family members to help care for your loved one with dementia?

f) Dealing with disagreements among family members about how to help your loved one with dementia?

g) Getting family or friends to accept that your loved one with dementia has memorv nroblems?

h) Getting treatments your loved one with dementia needs?

i) Try things that may prevent your loved one with dementia's memory problems from getting worse?

j) Things your loved one with dementia can do to help stay healthy?

k) Tests for diagnosing your loved one with dementia's memory problems?

1) Getting information about your loved one with dementia's memory

m) Understanding the causes of your loved one with dementia's memory

n) How to deal with other health conditions or problems he/shehas?

o) Making plans for how to deal with future changes in your loved one with dementia's memory problems?

p) Seeing that your loved one with dementia takes his/her medications in the correct amounts and at the correct times?

q) The way your loved one with dementia's medications are supposed to help?

r) The possible side effects of medications?

s) Knowing the future course of your loved one with dementia's memory

t) Keeping written notes to use when talking with your loved one with dementia's doctors or other service providers?

u) Getting instructions on how to care for your loved one with dementia's health conditions?

v) Scheduling follow-up visits with your loved one with dementia's doctors?

w) How to best manage your loved one with dementia's daily chores, such as shopping or doing laundry?

\begin{tabular}{|c|c|}
\hline NO & YES \\
\hline 0 & 1 \\
\hline 0 & 1 \\
\hline 0 & 1 \\
\hline 0 & 1 \\
\hline 0 & 1 \\
\hline 0 & 1 \\
\hline 0 & 1 \\
\hline 0 & 1 \\
\hline 0 & 1 \\
\hline 0 & 1 \\
\hline 0 & 1 \\
\hline 0 & 1 \\
\hline 0 & 1 \\
\hline 0 & 1 \\
\hline 0 & 1 \\
\hline 0 & 1 \\
\hline 0 & 1 \\
\hline 0 & 1 \\
\hline 0 & 1 \\
\hline 0 & 1 \\
\hline 0 & 1 \\
\hline 0 & 1 \\
\hline 0 & 1 \\
\hline
\end{tabular}




\begin{tabular}{|c|c|c|}
\hline Do you need more information about or help with this? & NO & YES \\
\hline $\begin{array}{l}\text { x) How to best manage your loved one with dementia's personal care such } \\
\text { as bathing and dressing? }\end{array}$ & 0 & 1 \\
\hline y) Ways to make the place where your loved one with dementia lives safe? & 0 & 1 \\
\hline $\begin{array}{l}\text { z) Getting someone to stay with your loved one with dementia so he/she } \\
\text { would not be left alone? }\end{array}$ & 0 & 1 \\
\hline aa) Whether your loved one with dementia should stop driving a car? & 0 & 1 \\
\hline bb) Your getting a break from caring for yourloved one with dementia? & 0 & 1 \\
\hline $\begin{array}{l}\text { cc) Finding services that care for your loved one with dementia when } \\
\text { family are not available? }\end{array}$ & 0 & 1 \\
\hline $\begin{array}{l}\text { dd) How to manage your loved one with dementia's finances such as bank } \\
\text { accounts and paying bills? }\end{array}$ & 0 & 1 \\
\hline $\begin{array}{l}\text { ee) Dealing with legal issues related to your loved one with dementia's } \\
\text { illness such as updating a will? }\end{array}$ & 0 & 1 \\
\hline $\begin{array}{l}\text { ff) Finding out what your loved one with dementia wants if he/she is unable to } \\
\text { speak for him/herself? }\end{array}$ & 0 & 1 \\
\hline gg) Having a living will, advance directives, healthcare durable power of & 0 & 1 \\
\hline hh) Understanding the help available from the VA? & 0 & 1 \\
\hline ii) Understanding Medicare or Medicaid? & 0 & 1 \\
\hline jj) Paying for services not covered by insurances? & 0 & 1 \\
\hline kk) Finding someone to talk with who understands your situation? & 0 & 1 \\
\hline $\begin{array}{l}\text { 11) Finding someone for your loved one with dementia to talk with who } \\
\text { understands his/her situation? }\end{array}$ & 0 & 1 \\
\hline mm) Getting emotional support or counseling for your loved one with & 0 & 1 \\
\hline nn) Getting emotional support or counseling for you? & 0 & 1 \\
\hline oo) Knowing which service providers to ask for different types of help? & 0 & 1 \\
\hline $\begin{array}{l}\text { pp) Services offered by the County Area Agency on Aging/ state chapter of } \\
\text { the Alzheimer's Association? }\end{array}$ & 0 & 1 \\
\hline qq) Getting your loved one with dementia to accept help from service & 0 & 1 \\
\hline rr) Getting different service providers to work together? & 0 & 1 \\
\hline ss) Finding services you or your loved one with dementia needs? & 0 & 1 \\
\hline tt) Getting transportation to locations where services are provided? & 0 & 1 \\
\hline $\begin{array}{l}\text { uu) Knowing the different places where your loved one with dementia could } \\
\text { live if he/she needed to move from his/her current home? }\end{array}$ & 0 & 1 \\
\hline vv) Paying for assisted living or nursing home care? & 0 & 1 \\
\hline ww) Getting information about assisted living facilities or nursing homes? & 0 & 1 \\
\hline
\end{tabular}


In the past month, did you use the following services? (Check all that apply)

Homemaker (paid person to help with cleaning, cooking, household chores)?

Home health services (Nurse, Occupational therapy, Physical therapy,

Respiratory therapy)

Visiting Nurse

Meals delivered to home (example: Meals on Wheels)

Transportation services (cab, ambulance, bus, etc.)

Adult Day Care

Support Services

Veteran Services

Help with transportation (unpaid person gave you and/or loved one with dementia a ride to an appointment, store, errands, etc.)

Informal emotional support (friend or family member showed compassion and concern for your well-being)

Informational support from friend or family member

Friend "just listened"

Friend or family member offered suggestions to you to help with caregiving Are the following services available in your area, and do you use the service? 


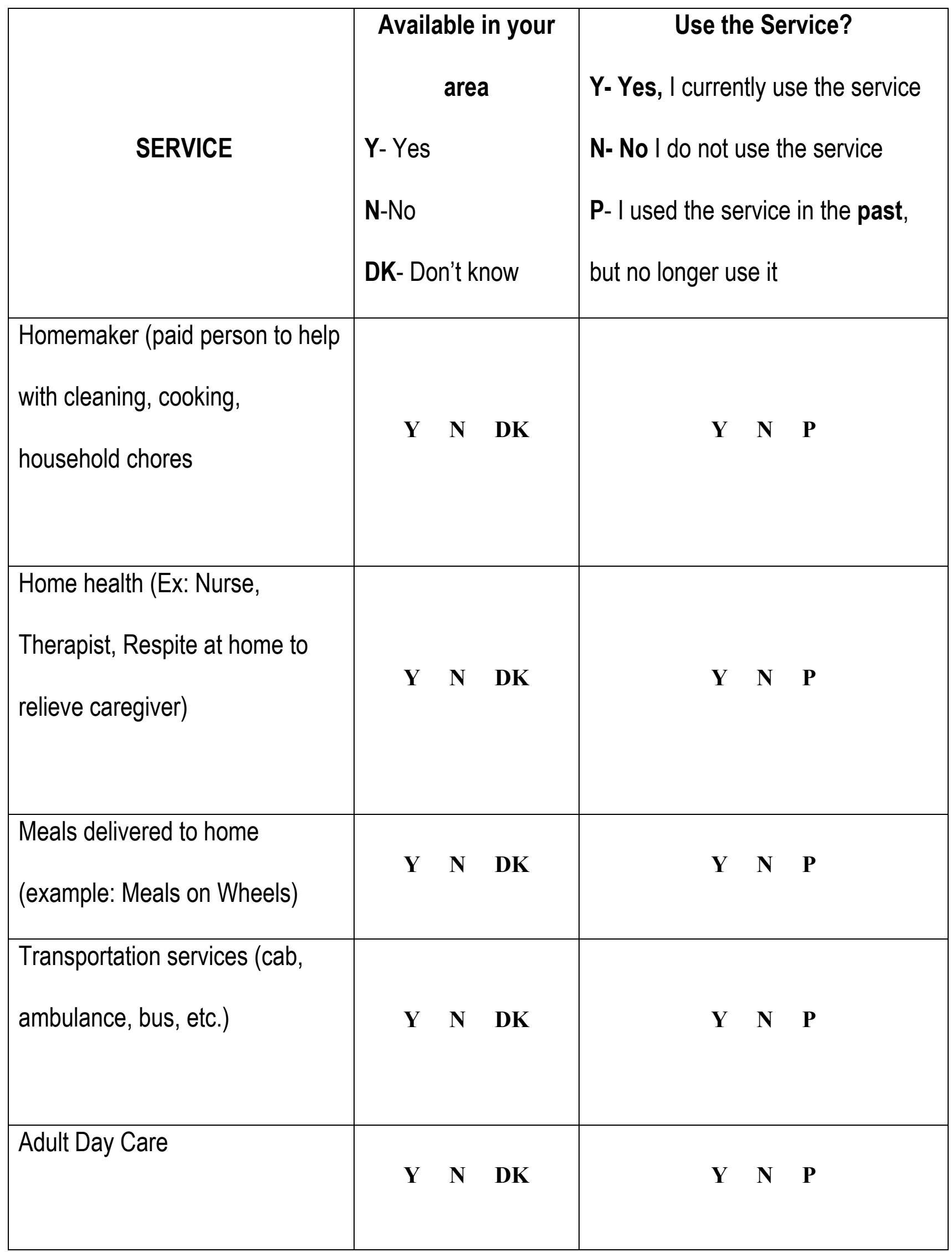




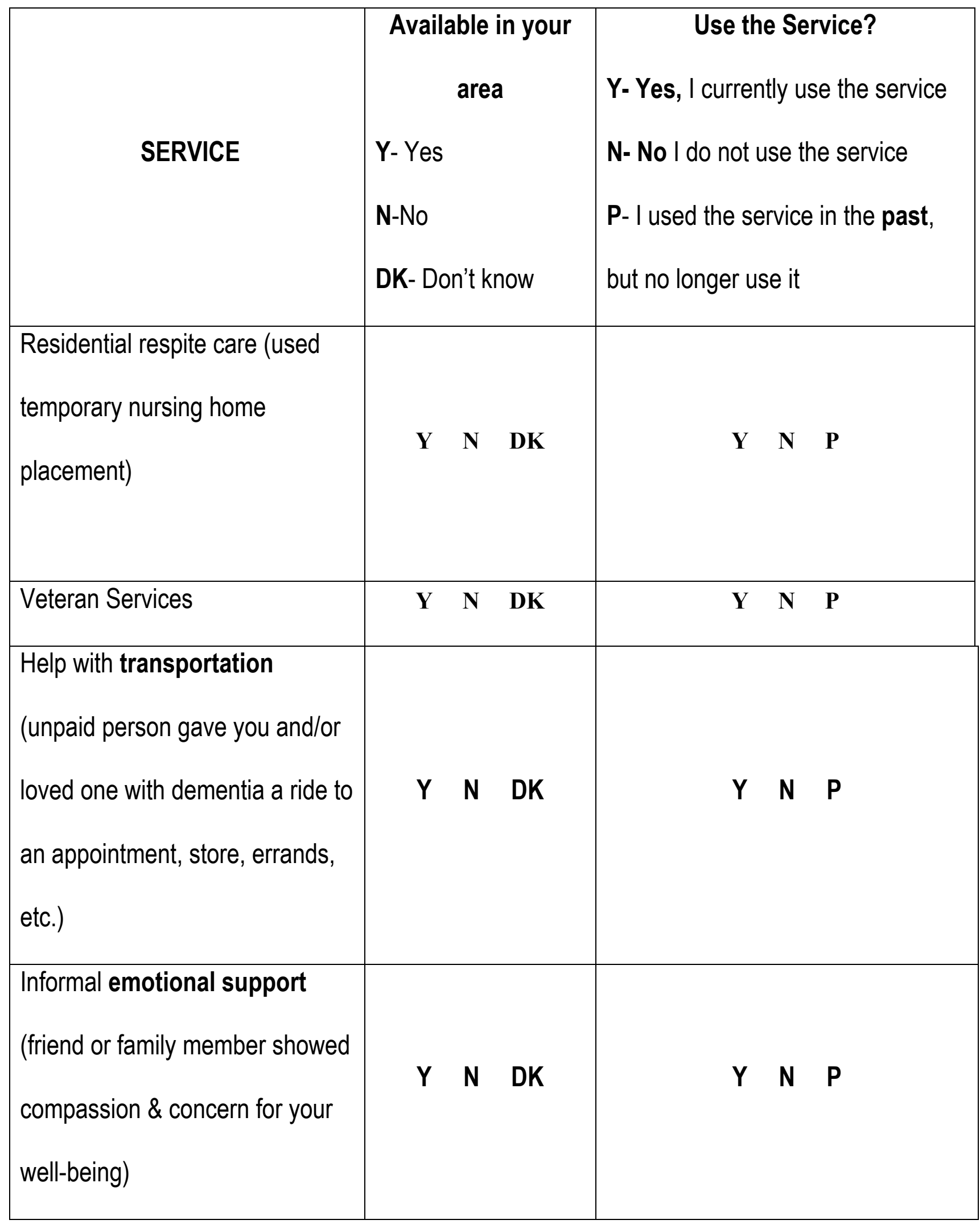




\begin{tabular}{|c|c|c|c|}
\hline SERVICE & $\begin{array}{l}\text { Availab } \\
\text { Y-Yes } \\
\text { N-No } \\
\text { DK- Don }\end{array}$ & $\begin{array}{l}\text { ble in your } \\
\text { area } \\
\text { 't know }\end{array}$ & $\begin{array}{l}\text { Use the Service? } \\
\text { Y- Yes, I currently use the service } \\
\text { N- No I do not use the service } \\
\text { P- I used the service in the past, } \\
\text { but no longer use it }\end{array}$ \\
\hline $\begin{array}{l}\text { Friend or family member offered } \\
\text { suggestions to you to help with } \\
\text { caregiving }\end{array}$ & Y & N DK & Y \\
\hline Friend "just listened" & $\mathbf{Y}$ & $\mathrm{N} \quad \mathrm{DK}$ & $\begin{array}{lll}Y & N & P\end{array}$ \\
\hline $\begin{array}{l}\text { Informational support from } \\
\text { friend or family member }\end{array}$ & $\mathbf{Y}$ & $\mathrm{N}$ DK & $\mathbf{Y}$ \\
\hline $\begin{array}{l}\text { Friend or family shared } \\
\text { caregiving responsibilities with } \\
\text { you (provided respite- "gave me } \\
\text { a break") }\end{array}$ & & N DK & $\mathbf{N}$ \\
\hline
\end{tabular}

Comments: 
Please read the statement and put a check in the box that says how often the sentence applies to you on a scale of 1 - Never to 5- Nearly Always

\begin{tabular}{|c|c|c|c|c|c|}
\hline Statement & $\begin{array}{c}\text { Never } \\
1\end{array}$ & $\begin{array}{c}\text { Rarely } \\
2\end{array}$ & $\begin{array}{c}\text { Sometimes } \\
3\end{array}$ & $\begin{array}{c}\text { Quite } \\
\text { Frequently } \\
4\end{array}$ & $\begin{array}{c}\text { Nearly } \\
\text { Always } \\
5\end{array}$ \\
\hline $\begin{array}{l}\text { No matter how much I do, } \\
\text { somehow, I feel guilty } \\
\text { about not doing enough for } \\
\text { the person with dementia. }\end{array}$ & & & & & \\
\hline $\begin{array}{l}\text { How often do you feel } \\
\text { uncertain about what to do } \\
\text { about the person with } \\
\text { dementia? }\end{array}$ & & & & & \\
\hline $\begin{array}{l}\text { How often do you feel you } \\
\text { should be doing more for } \\
\text { the person with dementia? }\end{array}$ & & & & & \\
\hline $\begin{array}{l}\text { How often do you feel that } \\
\text { you could do a better job in } \\
\text { caring for the person with } \\
\text { dementia? }\end{array}$ & & & & & \\
\hline
\end{tabular}


After reading the following statements about dementia, please rate your belief about the statement on a scale of 1-Strongly Disagree to 4- Strongly Agree

\begin{tabular}{|c|c|c|c|c|}
\hline Statement & $\begin{array}{c}\text { Strongly } \\
\text { Disagree } \\
1\end{array}$ & $\begin{array}{c}\text { Disagree } \\
2\end{array}$ & $\begin{array}{c}\text { Agree } \\
\\
3\end{array}$ & $\begin{array}{c}\text { Strongly } \\
\text { Agree } \\
4\end{array}$ \\
\hline $\begin{array}{l}\text { It is embarrassing taking the person } \\
\text { with dementia out in public. }\end{array}$ & & & & \\
\hline $\begin{array}{l}\text { I am self-conscious when in public } \\
\text { with the person with dementia. }\end{array}$ & & & & \\
\hline $\begin{array}{l}\text { I avoid company at home when the } \\
\text { person with dementia is there. }\end{array}$ & & & & \\
\hline $\begin{array}{l}\text { It is easy to have guests in the home } \\
\text { when the person with dementia is there. }\end{array}$ & & & & \\
\hline Dementia is a type of mental illness. & & & & \\
\hline $\begin{array}{l}\text { I feel embarrassed about the person } \\
\text { with dementia's memory problems }\end{array}$ & & & & \\
\hline $\begin{array}{l}\text { I feel embarrassed about the person } \\
\text { with dementia's behavior problems }\end{array}$ & & & & \\
\hline $\begin{array}{l}\text { Early treatment can delay progression } \\
\text { of dementia. }\end{array}$ & & & & \\
\hline
\end{tabular}




\begin{tabular}{|c|c|c|c|c|}
\hline Statement & $\begin{array}{l}\text { Strongly } \\
\text { Disagree } \\
1\end{array}$ & $\begin{array}{c}\text { Disagree } \\
2\end{array}$ & $\begin{array}{c}\text { Agree } \\
\\
3\end{array}$ & $\begin{array}{c}\text { Strongly } \\
\text { Agree } \\
4\end{array}$ \\
\hline $\begin{array}{l}\text { Delaying treatment has negative effect } \\
\text { on dementia progression. }\end{array}$ & & & & \\
\hline $\begin{array}{l}\text { Medication slows deterioration in } \\
\text { dementia. }\end{array}$ & & & & \\
\hline $\begin{array}{l}\text { Effective treatments are available for } \\
\text { dementia. }\end{array}$ & & & & \\
\hline $\begin{array}{l}\text { A variety of treatments are available } \\
\text { for dementia. }\end{array}$ & & & & \\
\hline $\begin{array}{l}\text { The government should provide more } \\
\text { money for respite programs and } \\
\text { services to assist caregivers. }\end{array}$ & & & & \\
\hline $\begin{array}{l}\text { The government should help families } \\
\text { care for persons at home }\end{array}$ & & & & \\
\hline $\begin{array}{l}\text { Service use may lead to the person with } \\
\text { dementia deterioration. }\end{array}$ & & & & \\
\hline
\end{tabular}




\begin{tabular}{|l|c|c|c|c|}
\hline \multicolumn{1}{|c|}{ Statement } & Strongly & Disagree & Agree & Strongly \\
\hline pervice use may cause conflict with the & & Disagree & & Agree \\
\hline Service use may provide no benefit to & & & & \\
the person with dementia. & & & & \\
\hline My family member or friend with & & & & \\
dementia refuses or does not want to & & & & \\
attend a day center. & & & & \\
\hline I do not know how to access services. & & & & \\
\hline Services are not available when needed & & & & \\
\hline loved one with dementia physical or & & & & \\
\hline Services are not close to where I live & & & & \\
\hline Service waiting times are too long & & & & \\
\hline The service cannot provide for my & & & & \\
\hline
\end{tabular}


If there is anything you would like to say about the reason you have not used resources/supports to help you care for the person with dementia, please share your thoughts, concerns, or comments below.

Please return completed survey with SIGNED consent form in self- addressed stamped envelope provided. Thank you for taking your valuable time. 
Appendix J Tables 6.1 Q1A - 6.12 Q4C Correlations and Linear Regressions from Research Questions

Table 6.1. Question (Q) A: Predisposing characteristic variables related to non-use of both services in Appalachia (N=40)

\begin{tabular}{|c|c|c|c|c|c|c|c|c|c|c|c|}
\hline Variable & & & & & Pear & & & & & $\beta$ & Adjusted \\
\hline & $\begin{array}{l}\text { Beliefs } \\
\text { Services }\end{array}$ & $\begin{array}{l}\text { Beliefs } \\
\text { Dementia }\end{array}$ & SPS & Faith & $\begin{array}{l}\text { CG } \\
\text { relat. } \\
\text { PWD }\end{array}$ & $\begin{array}{l}\text { CG } \\
\text { Level of } \\
\text { Ed. }\end{array}$ & $\begin{array}{l}\text { CG } \\
\text { Age } \\
\text { Yrs }\end{array}$ & $\begin{array}{l}\text { Gender } \\
\mathrm{CG}\end{array}$ & $\begin{array}{l}\text { Non-use of } \\
\text { Formal }+ \\
\text { Informal } \\
\text { Services(14 }\end{array}$ & & \\
\hline Gender CG & & & & & & & & & -.065 & & \\
\hline $\begin{array}{l}\text { CG Age } \\
\text { Years }\end{array}$ & & & & & & & & .104 & .130 & & \\
\hline $\begin{array}{l}\text { CG Level } \\
\text { Education }\end{array}$ & & & & & & & $-.307 *$ & -.048 & -.022 & & \\
\hline $\begin{array}{l}\text { CG relat. } \\
\text { PWD }\end{array}$ & & & & & & -.178 & -.108 & .034 & -.139 & & \\
\hline
\end{tabular}




\begin{tabular}{|c|c|c|c|c|c|c|c|c|c|c|c|}
\hline $\begin{array}{l}\text { Faith } \\
\text { decisions }\end{array}$ & & & & & .136 & .020 & $.338 *$ & .028 & .008 & & \\
\hline SPS & & & & .120 & .060 & .232 & -.044 & $.304 *$ & $-.327 *$ & -.139 & $.083 * *$ \\
\hline $\begin{array}{l}\text { Beliefs } \\
\text { Dementia }\end{array}$ & & & -.223 & -.150 & -.064 & .050 & .047 & .144 & -.110 & & \\
\hline $\begin{array}{l}\text { Beliefs } \\
\text { Services }\end{array}$ & & $.812 *$ & -.133 & -.214 & .021 & -.021 & -.108 & .003 & -.158 & & \\
\hline Mean & 54.33 & 28.05 & 30.55 & .80 & 2.70 & 3.55 & 61.78 & .28 & 8.90 & & \\
\hline SD & 7.20 & 5.30 & 3.91 & .41 & 2.14 & 1.24 & 11.66 & .45 & 1.66 & & \\
\hline
\end{tabular}

$* p<.05 \quad * * \mathrm{p}=.039$ 
Appendix J Table 6.2 Q1B Predisposing characteristic variables related to non-use of formal services in Appalachia (N=40)

\begin{tabular}{|c|c|c|c|c|c|c|c|c|c|c|c|}
\hline Variable & & & & & Pea & & & & & $\beta$ & Adjusted \\
\hline & $\begin{array}{l}\text { Beliefs } \\
\text { Services }\end{array}$ & $\begin{array}{l}\text { Beliefs } \\
\text { Dementia }\end{array}$ & SPS & Faith & $\begin{array}{l}\text { CG } \\
\text { relat. } \\
\text { PWD }\end{array}$ & $\begin{array}{l}\text { CG } \\
\text { Level of } \\
\text { Ed. }\end{array}$ & $\begin{array}{l}\text { CG } \\
\text { Age } \\
\text { Yrs }\end{array}$ & $\begin{array}{l}\text { Gender } \\
\mathrm{CG}\end{array}$ & $\begin{array}{l}\text { Non-Use } \\
\text { Formal } \\
\text { Services } \\
\text { (8) }\end{array}$ & & $\begin{array}{l}\text { ariables } \\
\text { red into } \\
\text { uation }\end{array}$ \\
\hline Gender CG & & & & & & & & & -.039 & & \\
\hline $\begin{array}{l}\text { CG Age } \\
\text { Years }\end{array}$ & & & & & & & & .104 & -.159 & & \\
\hline $\begin{array}{l}\text { CG Level } \\
\text { Education }\end{array}$ & & & & & & & $-.307^{*}$ & -.048 & .145 & & \\
\hline $\begin{array}{l}\text { CG relat. } \\
\text { PWD }\end{array}$ & & & & & & -.178 & -.108 & .034 & .107 & & \\
\hline $\begin{array}{l}\text { Faith } \\
\text { decisions }\end{array}$ & & & & & .136 & .020 & $.338^{*}$ & .028 & .106 & & \\
\hline
\end{tabular}




\begin{tabular}{|c|c|c|c|c|c|c|c|c|c|}
\hline SPS & & & & .120 & .060 & .232 & -.044 & $.304 *$ & -.014 \\
\hline $\begin{array}{l}\text { Beliefs } \\
\text { Dementia }\end{array}$ & & & -.223 & -.150 & -.064 & .050 & .047 & .144 & $-.293 *$ \\
\hline $\begin{array}{l}\text { Beliefs } \\
\text { Services }\end{array}$ & & $.812 *$ & -.133 & -.214 & .021 & -.021 & -.108 & .003 & -.070 \\
\hline Mean & 54.33 & 28.05 & 30.55 & .80 & 2.70 & 3.55 & 61.78 & .28 & 6.90 \\
\hline $\mathrm{SD}$ & 7.20 & 5.30 & 3.91 & .41 & 2.14 & 1.24 & 11.66 & .45 & 1.32 \\
\hline
\end{tabular}

No variables loaded into the equation. 
Appendix J Table 6.3 Q1C Predisposing characteristic variables related to non-use of informal services in Appalachia (N=40)

\begin{tabular}{|c|c|c|c|c|c|c|c|c|c|c|c|}
\hline Variable & & & & & Pear & & & & & $\beta$ & Adjusted \\
\hline & $\begin{array}{l}\text { Beliefs } \\
\text { Services }\end{array}$ & $\begin{array}{l}\text { Beliefs } \\
\text { Dementia }\end{array}$ & SPS & Faith & $\begin{array}{l}\text { CG } \\
\text { relat. } \\
\text { PWD }\end{array}$ & $\begin{array}{l}\text { CG } \\
\text { Level of } \\
\text { Ed. }\end{array}$ & $\begin{array}{l}\text { CG } \\
\text { Age } \\
\text { Yrs }\end{array}$ & $\begin{array}{l}\text { Gender } \\
\mathrm{CG}\end{array}$ & $\begin{array}{l}\text { Non-Use } \\
\text { Informal } \\
\text { Services } \\
\text { (6) }\end{array}$ & & \\
\hline Gender CG & & & & & & & & & -.034 & & \\
\hline $\begin{array}{l}\text { CG Age } \\
\text { Years }\end{array}$ & & & & & & & & .104 & .255 & & \\
\hline $\begin{array}{l}\text { CG Level } \\
\text { Education }\end{array}$ & & & & & & & $-.307^{*}$ & -.048 & -.137 & & \\
\hline $\begin{array}{l}\text { CG relat. } \\
\text { PWD }\end{array}$ & & & & & & -.178 & -.108 & .034 & -.223 & & \\
\hline $\begin{array}{l}\text { Faith } \\
\text { decisions }\end{array}$ & & & & & .136 & .020 & $.338^{*}$ & .028 & -.076 & & \\
\hline
\end{tabular}




\begin{tabular}{|c|c|c|c|c|c|c|c|c|c|c|c|}
\hline SPS & & & & .120 & .060 & .232 & -.044 & $.304^{*}$ & $-.315^{*}$ & -.134 & $.076^{* *}$ \\
\hline $\begin{array}{l}\text { Beliefs } \\
\text { Dementia }\end{array}$ & & & -.223 & -.150 & -.064 & .050 & .047 & .144 & .122 & & \\
\hline $\begin{array}{l}\text { Beliefs } \\
\text { Services }\end{array}$ & & $.812 *$ & -.133 & -.214 & .021 & -.021 & -.108 & .003 & -.103 & & \\
\hline Mean & 54.33 & 28.05 & 30.55 & .80 & 2.70 & 3.55 & 61.78 & .28 & 2.00 & & \\
\hline SD & 7.20 & 5.30 & 3.91 & .41 & 2.14 & 1.24 & 11.66 & .45 & 1.32 & & \\
\hline
\end{tabular}

$* p<.05 \quad * * p=.047$ 
Appendix J Table 6.4 Q2A Enabling characteristic variables related to non-use of both services in Appalachia (N=38)

\begin{tabular}{|c|c|c|c|c|c|c|c|}
\hline Variable & & & Pearso & & & $\beta$ & Adj. R \\
\hline & $\begin{array}{l}\text { Enabling } \\
\text { Organizing }\end{array}$ & $\begin{array}{l}\text { Legal \& } \\
\text { Financial }\end{array}$ & $\begin{array}{l}\text { Household } \\
\text { income }\end{array}$ & $\begin{array}{l}\text { CGS } \\
\text { Available }\end{array}$ & $\begin{array}{c}\text { Non-use of } \\
\text { Formal }+ \\
\text { Informal } \\
\text { Services (14) }\end{array}$ & & \\
\hline CGS Available & & & & & $.375 *$ & $.173 * *$ & .117 \\
\hline Household income & & & & -.212 & -.186 & & \\
\hline Legal \& Financial & & & -.239 & .050 & .163 & & \\
\hline $\begin{array}{l}\text { Enabling } \\
\text { Organizing }\end{array}$ & & $.610^{*}$ & -.149 & .153 & -.050 & & \\
\hline Mean & 5.39 & 2.18 & 3.21 & 17.66 & 8.97 & & \\
\hline SD & 4.21 & 2.20 & 1.56 & 3.78 & 1.75 & & \\
\hline
\end{tabular}

$* p<.05 \quad * * p=.020$

Enabling Organizing Unmet Needs Assessment Family Concerns, Emotional Support, and Finding Services= Enabling Organizing

Total Unmet Needs Assessment Legal and Financial= Legal and Financial

Total formal and informal Caregiving Services Available= CGS Available 
Appendix J Table 6.5 Q2B Enabling characteristic variables related to non-use of formal services in Appalachia (N=38)

\begin{tabular}{|c|c|c|c|c|c|c|c|}
\hline Variable & & & Pearso & & & $\beta$ & Adj. R \\
\hline & $\begin{array}{l}\text { Enabling } \\
\text { Organizing }\end{array}$ & $\begin{array}{l}\text { Legal \& } \\
\text { Financial }\end{array}$ & $\begin{array}{l}\text { Household } \\
\text { income }\end{array}$ & $\begin{array}{l}\text { CGS } \\
\text { Available }\end{array}$ & $\begin{array}{c}\text { Non-Use } \\
\text { Formal } \\
\text { Services (8) }\end{array}$ & & \\
\hline CGS Available & & & & & -.023 & & \\
\hline Household income & & & & -.212 & .062 & & \\
\hline Legal \& Financial & & & -.239 & .050 & -.039 & & \\
\hline $\begin{array}{l}\text { Enabling } \\
\text { Organizing }\end{array}$ & & $.610^{*}$ & -.149 & .153 & -.140 & -.060 & -.092 \\
\hline Mean & 5.39 & 2.18 & 3.21 & 17.66 & 6.89 & & \\
\hline SD & 4.21 & 2.20 & 1.56 & 3.78 & 1.35 & & \\
\hline
\end{tabular}

$* p<.05$

Nothing was significant in the model with Dependent variable Non-use Formal Services. The variables enabling organizing and Legal and Financial are correlated $(\mathrm{r}=.610 ; p=.000)$. 
Appendix J Table 6.6 Q2C Enabling characteristic variables related to non-use of informal services in Appalachia (N=38)

\begin{tabular}{|c|c|c|c|c|c|c|c|}
\hline Variable & & & Pearso & & & $\beta$ & Adj. R \\
\hline & $\begin{array}{l}\text { Enabling } \\
\text { Organizing }\end{array}$ & $\begin{array}{l}\text { Legal \& } \\
\text { Financial }\end{array}$ & $\begin{array}{l}\text { Household } \\
\text { income }\end{array}$ & $\begin{array}{l}\text { CGS } \\
\text { Available }\end{array}$ & $\begin{array}{l}\text { Non-Use } \\
\text { Informal } \\
\text { Services (6) }\end{array}$ & & \\
\hline CGS Available & & & & & $.401 *$ & $.176^{* *}$ & .137 \\
\hline Household income & & & & -.212 & -.238 & & \\
\hline Legal \& Financial & & & -.239 & .050 & .196 & & \\
\hline $\begin{array}{l}\text { Enabling } \\
\text { Organizing }\end{array}$ & & $.610^{*}$ & -.149 & .153 & .059 & & \\
\hline Mean & 5.39 & 2.18 & 3.21 & 17.66 & 2.08 & & \\
\hline SD & 4.21 & 2.20 & 1.56 & 3.78 & 1.72 & & \\
\hline
\end{tabular}

${ }^{*} p<.05 \quad * * p=.020$ 
Appendix J Table 6.7 Q3A Need characteristic variables related to non-use of both services in Appalachia $(\mathrm{N}=42)$

\begin{tabular}{|c|c|c|c|c|c|c|}
\hline \multirow[t]{2}{*}{ Variable } & \multicolumn{4}{|c|}{ Pearson $r$} & \multirow[t]{2}{*}{$\beta$} & \multirow{2}{*}{$\begin{array}{l}\text { Adj. R } \\
\text { Square }\end{array}$} \\
\hline & $\begin{array}{c}\text { Total } \\
\text { Caregiver } \\
\text { Mastery } \\
\text { Scores }\end{array}$ & $\begin{array}{l}\text { Perceived } \\
\text { Need }\end{array}$ & $\begin{array}{c}\text { Evaluated } \\
\text { Need }\end{array}$ & $\begin{array}{c}\text { Non-use of } \\
\text { Formal }+ \\
\text { Informal } \\
\text { Services (14) }\end{array}$ & & \\
\hline Evaluated Need & & & & $-.319 *$ & $-.031 * *$ & .079 \\
\hline Perceived Need & & & .067 & -.172 & & \\
\hline $\begin{array}{l}\text { Total Caregiver Mastery } \\
\text { Scores }\end{array}$ & & .200 & -.141 & -.175 & & \\
\hline Mean & 12.81 & 9.29 & 16.93 & 9.05 & & \\
\hline SD & 3.33 & 6.83 & 18.03 & 1.74 & & \\
\hline
\end{tabular}

$* p<.05 * * \mathrm{p}=.040$ 
Appendix J Table 6.8 Q3B Need characteristic variables related to non-use of formal services in Appalachia (N=42)

\begin{tabular}{|c|c|c|c|c|c|c|}
\hline \multirow[t]{2}{*}{ Variable } & \multicolumn{4}{|c|}{ Pearson $r$} & \multirow[t]{2}{*}{$\beta$} & \multirow{2}{*}{$\begin{array}{l}\text { Adj. R } \\
\text { Square }\end{array}$} \\
\hline & $\begin{array}{c}\text { Total } \\
\text { Caregiver } \\
\text { Mastery } \\
\text { Scores }\end{array}$ & $\begin{array}{l}\text { Perceived } \\
\text { Need }\end{array}$ & $\begin{array}{c}\text { Evaluated } \\
\text { Need }\end{array}$ & $\begin{array}{c}\text { Non-use of } \\
\text { Formal } \\
\text { Services (8) }\end{array}$ & & \\
\hline Evaluated Need & & & & $-.522 *$ & $-.038 * *$ & .264 \\
\hline Perceived Need & & & .067 & -.220 & & \\
\hline $\begin{array}{l}\text { Total Caregiver Mastery } \\
\text { Scores }\end{array}$ & & .200 & -.141 & -.064 & & \\
\hline Mean & 12.81 & 9.29 & 16.93 & 6.95 & & \\
\hline SD & 3.33 & 6.83 & 18.03 & 1.31 & & \\
\hline
\end{tabular}

$* p<.05 * * p=.002$ 
Appendix J Table 6.9 Q3C Need characteristic variables related to non-use of informal services in Appalachia $(\mathrm{N}=42)$

\begin{tabular}{|c|c|c|c|c|c|c|}
\hline \multirow[t]{2}{*}{ Variable } & \multicolumn{4}{|c|}{ Pearson $r$} & \multirow[t]{2}{*}{$\beta$} & \multirow{2}{*}{$\begin{array}{l}\text { Adj. R } \\
\text { Square }\end{array}$} \\
\hline & $\begin{array}{c}\text { Total } \\
\text { Caregiver } \\
\text { Mastery } \\
\text { Scores }\end{array}$ & $\begin{array}{c}\text { Perceived } \\
\text { Need }\end{array}$ & $\begin{array}{c}\text { Evaluated } \\
\text { Need }\end{array}$ & $\begin{array}{c}\text { Non-use of } \\
\text { Informal } \\
\text { Services (6) }\end{array}$ & & \\
\hline Evaluated Need & & & & .075 & & \\
\hline Perceived Need & & & .067 & -.007 & & \\
\hline $\begin{array}{l}\text { Total Caregiver Mastery } \\
\text { Scores }\end{array}$ & & .200 & -.141 & -.131 & & \\
\hline Mean & 12.81 & 9.29 & 16.93 & 2.10 & & \\
\hline SD & 3.33 & 6.83 & 18.03 & 1.69 & & \\
\hline
\end{tabular}

$* p<.05$

Nothing significantly correlated and nothing significant entered into the model. 
Appendix J Table 6.10 Q4A Strongest explanatory model of non-use of services both services $(\mathrm{N}=42)$

\begin{tabular}{|c|c|c|c|c|c|c|}
\hline \multirow[t]{2}{*}{ Variable } & \multicolumn{4}{|c|}{ Pearson $r$} & \multirow[t]{2}{*}{$\beta$} & \multirow{2}{*}{$\begin{array}{l}\text { Adj. R } \\
\text { Square }\end{array}$} \\
\hline & $\begin{array}{l}\text { Evaluated } \\
\text { Need }\end{array}$ & $\begin{array}{l}\text { Caregiving } \\
\text { Services } \\
\text { Available }\end{array}$ & $\begin{array}{l}\text { Total SPS } \\
\text { Scale Score }\end{array}$ & $\begin{array}{l}\text { Non-use of } \\
\text { Formal }+ \\
\text { Informal } \\
\text { Services } \\
\text { (14) }\end{array}$ & & \\
\hline Total SPS Scale Score & & & & $-.350^{*}$ & & \\
\hline Caregiving Services Available & & & -.212 & $.454^{*}$ & $.210 * *$ & .187 \\
\hline Evaluated Need & & .125 & .011 & $-.362 *$ & $-.041 * * *$ & .353 \\
\hline & & & & & & \\
\hline Mean & 16.83 & 17.93 & 30.52 & 9.00 & & \\
\hline SD & 17.89 & 4.12 & 3.92 & 1.74 & & \\
\hline
\end{tabular}

$* p<.05 \quad * * p=.000 \quad * * * \mathrm{p}=.002$ 
Appendix J Table 6.11 Q4B Strongest explanatory model of non-use of formal services $(\mathrm{N}=42)$

\begin{tabular}{|c|c|c|c|c|c|c|}
\hline \multirow[t]{2}{*}{ Variable } & \multicolumn{4}{|c|}{ Pearson $\mathrm{r}$} & \multirow[t]{2}{*}{$\beta$} & \multirow{2}{*}{$\begin{array}{l}\text { Adj. R } \\
\text { Square }\end{array}$} \\
\hline & $\begin{array}{l}\text { Evaluated } \\
\text { Need }\end{array}$ & $\begin{array}{l}\text { Caregiving } \\
\text { Services } \\
\text { Available }\end{array}$ & $\begin{array}{l}\text { Total SPS } \\
\text { Scale Score }\end{array}$ & $\begin{array}{l}\text { Non-use } \\
\text { Formal } \\
\text { Services (8) }\end{array}$ & & \\
\hline Total SPS Scale Score & & & & -.014 & & \\
\hline Caregiving Services Available & & & -.212 & .039 & & \\
\hline Evaluated Need & & .125 & .011 & $-.527 *$ & $-.038 * *$ & .259 \\
\hline Mean & 16.83 & 17.93 & 30.52 & 6.95 & & \\
\hline SD & 17.89 & 4.20 & 3.92 & 1.31 & & \\
\hline
\end{tabular}

$* p<.05 \quad * * p=.000$ 
Appendix J Table 6.12 Q4C Strongest explanatory model of non-use of informal services ( $\mathrm{N}=42)$

\begin{tabular}{|c|c|c|c|c|c|c|}
\hline \multirow[t]{2}{*}{ Variable } & \multicolumn{4}{|c|}{ Pearson $r$} & $\beta$ & Adj. R \\
\hline & $\begin{array}{l}\text { Evaluated } \\
\text { Need }\end{array}$ & $\begin{array}{l}\text { Caregiving } \\
\text { Services } \\
\text { Available }\end{array}$ & $\begin{array}{l}\text { Total SPS } \\
\text { Scale Score }\end{array}$ & $\begin{array}{l}\text { Non-use } \\
\text { Formal } \\
\text { Services (8) }\end{array}$ & & \\
\hline Total SPS Scale Score & & & & $-.349 *$ & & \\
\hline Caregiving Services Available & & & -.212 & $.436^{*}$ & $.176^{* *}$ & .169 \\
\hline Evaluated Need & & .125 & .011 & .034 & & \\
\hline Mean & 16.83 & 17.93 & 30.52 & 2.05 & & \\
\hline SD & 17.89 & 4.20 & 3.92 & 1.70 & & \\
\hline
\end{tabular}

$* p<.05 \quad * * p=.004$ 
Appendix K Table 7 Inclusion and Exclusion Criteria for Study

\begin{tabular}{|c|c|}
\hline Inclusion Criteria & Exclusion Criteria \\
\hline $\begin{array}{l}\text { Informal (unpaid) CG will have a family to } \\
\text { the CR (PWD) }\end{array}$ & $\begin{array}{l}\text { Formal (paid) CG and/or } \\
\text { Non-familial relationship to CR (PWD) }\end{array}$ \\
\hline $\begin{array}{l}\text { CG/PWD reside within an Appalachian } \\
\text { designated county per the Appalachian } \\
\text { Regional Commission designation. }\end{array}$ & $\begin{array}{l}\text { Either the CG or PWD living outside an } \\
\text { Appalachian county as designated by the } \\
\text { Appalachian Regional Commission. }\end{array}$ \\
\hline $\begin{array}{l}\text { CG is age } 18 \text { years or older and } \\
\text { speaks/reads/comprehends written and spoken } \\
\text { English. }\end{array}$ & $\begin{array}{l}\text { CG or PWD below age } 18 \text { years and/or } \\
\text { unable to speak/read/comprehend written and } \\
\text { spoken English. }\end{array}$ \\
\hline $\begin{array}{l}\text { The PWD must be an adult over the age of } 18 \\
\text { years and be diagnosed with dementia (either } \\
\text { Alzheimer's or related dementia [ADRD]) by } \\
\text { a health care provider. (CG will self-report } \\
\text { diagnosis of dementia by a licensed health } \\
\text { care provider such as a physician, nurse } \\
\text { practitioner, physician's assistant). }\end{array}$ & $\begin{array}{l}\text { PWD has neurocognitive impairment known } \\
\text { not to be dementia (such as mass, traumatic } \\
\text { brain injury without dementia, etc.) or PWD } \\
\text { not diagnosed by a licensed health care } \\
\text { provider as having dementia. }\end{array}$ \\
\hline $\begin{array}{l}\text { The caregiver must assist the PWD in some } \\
\text { manner with household activities (e.g. } \\
\text { housework, meal preparation, supervising } \\
\text { outside services), personal care (e.g. hygiene, } \\
\text { grooming, toileting), financial management, }\end{array}$ & $\begin{array}{l}\text { The CG does not assist the PWD in any } \\
\text { manner with household activities (e.g. } \\
\text { housework, meal preparation, supervising } \\
\text { outside services), personal care (e.g. hygiene, } \\
\text { grooming, toileting), financial management, }\end{array}$ \\
\hline
\end{tabular}




\begin{tabular}{|l|l|}
\hline $\begin{array}{l}\text { shopping and transportation, emotional or } \\
\text { physical support (e.g. social activities, }\end{array}$ & shopping and transportation, emotional or \\
cheerful friend), or monitoring care (e.g. & physical support (e.g. social activities, \\
evaluating treatment outcomes, administering & evaluating treatment outcomes, administering \\
medications). & medications). \\
\hline $\begin{array}{l}\text { CR can live in assisted living or long-term } \\
\text { care facility, or not with CG (but both live in }\end{array}$ & \\
Appalachian designated county per & \\
Appalachian Regional Commission). & \\
\hline
\end{tabular}

CG-caregiver; CR- care recipient also known as Person with Dementia (PWD) 
Appendix L Map of Counties in WV and surrounding States with Respondent Counties

Identified

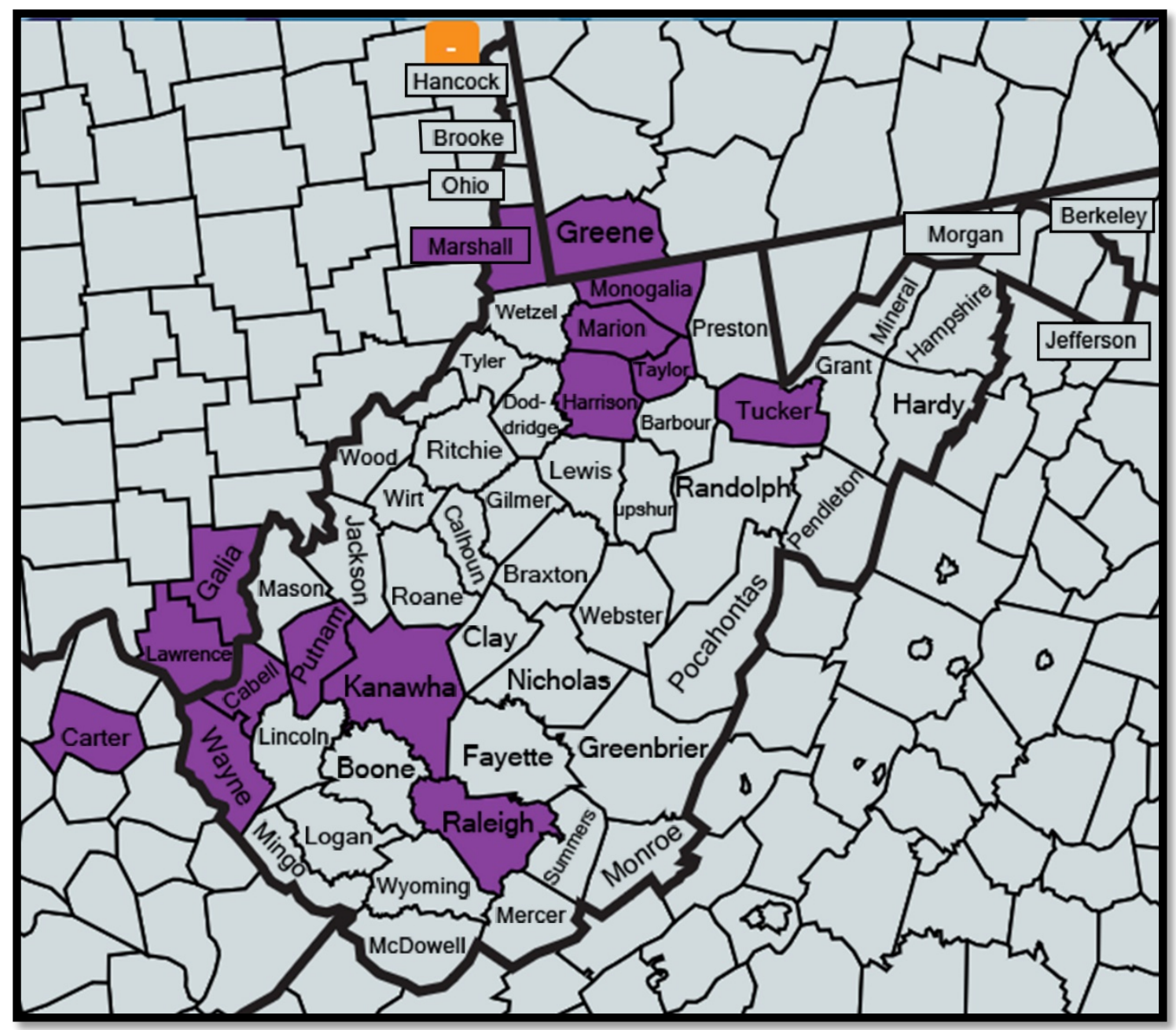


Appendix M All Questions Unmet Needs Assessment (UNA)

Benjamin Rose Institute Unmet Needs Assessment (Bass et al., 2012, 2013)

Please read the list of topics. After reading each topic, I would like you to tell me if you or your loved one with dementia need more information or help with this topic. Circle either No (0) or Yes (1).

\begin{tabular}{|c|c|c|}
\hline \multirow[t]{2}{*}{$\begin{array}{c}\text { UNMET NEED ASSESSMENT - BENJAMIN } \\
\text { ROSE INSTITUTE ON AGING }\end{array}$} & $\begin{array}{l}\text { B. 17. Do you need } \\
\text { more information } \\
\text { about or help with } \\
\text { this? }\end{array}$ & \\
\hline & NO & YES \\
\hline $\begin{array}{l}\text { FAMILY CONCERNS } \\
\text { a) Getting family members or friends to help your } \\
\text { loved one with dementia because of his/her } \\
\text { memory problems? }\end{array}$ & 0 & 1 \\
\hline $\begin{array}{l}\text { b) How to discuss your loved one with dementia's } \\
\text { memory problems with other family members? }\end{array}$ & 0 & 1 \\
\hline $\begin{array}{l}\text { c) Planning who would help your loved one with } \\
\text { dementia if you were no longer able to help? }\end{array}$ & 0 & 1 \\
\hline $\begin{array}{l}\text { d) Discussing the future course of your loved } \\
\text { one with dementia's illness with other family } \\
\text { members? }\end{array}$ & 0 & 1 \\
\hline $\begin{array}{l}\text { e) Getting family members to cooperate in helping } \\
\text { your loved one with dementia? }\end{array}$ & 0 & 1 \\
\hline $\begin{array}{l}\text { f) Dealing with disagreements among family } \\
\text { members about how to help your loved one } \\
\text { with dementia? }\end{array}$ & 0 & 1 \\
\hline $\begin{array}{l}\text { g) Getting family or friends to accept that your } \\
\text { loved one with dementia has memory } \\
\text { problems? }\end{array}$ & 0 & 1 \\
\hline HEALTH INFORMATION & & \\
\hline $\begin{array}{l}\text { h) Getting treatments your loved one with dementia } \\
\text { needs? }\end{array}$ & 0 & 1 \\
\hline $\begin{array}{l}\text { i) Try things that may prevent your loved one with } \\
\text { dementia's memory problems from getting } \\
\text { worse? }\end{array}$ & 0 & 1 \\
\hline
\end{tabular}




\begin{tabular}{|l|l|l|}
\hline $\begin{array}{l}\text { j) Things your loved one with dementia can do to } \\
\text { help stay healthy? }\end{array}$ & 0 & 1 \\
\hline $\begin{array}{l}\text { k) Tests for diagnosing your loved one with } \\
\text { dementia's memory problems? }\end{array}$ & 0 & 1 \\
\hline $\begin{array}{l}\text { 1) Getting information about your loved one with } \\
\text { dementia's memory problems? }\end{array}$ & 0 & 1 \\
\hline $\begin{array}{l}\text { m) Understanding the causes of your loved one with } \\
\text { dementia's memory problems? }\end{array}$ & 0 & 1 \\
\hline $\begin{array}{l}\text { n) How to deal with other health conditions or } \\
\text { problems he/shehas? }\end{array}$ & 0 & 1 \\
\hline $\begin{array}{l}\text { o) Making plans for how to deal with future } \\
\text { changes in yourloved one with dementia's } \\
\text { memory problems? }\end{array}$ & 0 & 1 \\
\hline $\begin{array}{l}\text { p) Seeing that your loved one with dementia } \\
\text { takes his/her medications in the correct } \\
\text { amounts and at the correct times? }\end{array}$ & 0 & 1 \\
\hline $\begin{array}{l}\text { q) The way your loved one with dementia's } \\
\text { medications are supposed to help? }\end{array}$ & 0 & 1 \\
\hline $\begin{array}{l}\text { r) The possible side effects of medications? } \\
\text { s) Knowing the future course of your loved one with } \\
\text { dementia's memory problems? }\end{array}$ & 0 & 1 \\
\hline $\begin{array}{l}\text { t) Keeping written notes to use when talking } \\
\text { with your loved one with dementia's doctors } \\
\text { or other service providers? }\end{array}$ & $\begin{array}{l}\text { your loved one with dementia's health } \\
\text { conditions? }\end{array}$ & 0 \\
\hline $\begin{array}{l}\text { v) Scheduling follow-up visits with your loved one } \\
\text { with dementia's doctors? }\end{array}$ & 0 & 1 \\
\hline
\end{tabular}


Benjamin Rose Institute Unmet Needs Assessment (Bass et al., 2012, 2013) Continued p. 3

Please read the list of topics. After reading each topic, I would like you to tell me if you or your loved one with

Dementia need more information or help with this topic. Circle either No (0) or Yes (1).

UNMET NEED ASSESSMENT - BENJAMIN ROSE INSTITUTE ON B. 17. Do you need more AGING

information about or help with thic?

DAILY LIVING TASKS

w) How to best manage your loved one with dementia's daily chores, such as shopping or doing laundry?

x) How to best manage your loved one with dementia's personal care such as bathing and dressing?

y) Ways to make the place where your loved one with dementia lives safe?

z) Getting someone to stay with your loved one with dementia so he/she would not be left alone?

aa) Whether your loved one with dementia should stop driving a car? RESPITE SERVICES

bb) Your getting a break from caring for your loved one with dementia?

cc) Finding services that care for your loved one with dementia when family are not available?

\section{LEGAL AND FINANCIAL ISSUES}

dd) How to manage your loved one with dementia 's finances such as bank accounts and paying bills?

ee) Dealing with legal issues related to your loved one with dementia 's illness such as updating a will?

ff) Finding out what your loved one with dementia wants if he/she is unable to speak for him/herself?

gg) Having a living will, advance directives, or durable power of attorney for health care?

hh) Understanding the help available from the VA?

ii) Understanding Medicare or Medicaid?

ji) Paying for services not covered by insurances?

\section{EMOTIONAL SUPPORT OR COUNSELING}

kk) Finding someone to talk with who understands your situation?

11) Finding someone for your loved one with dementia to talk with who understands his/her situation?

$\mathrm{mm}$ ) Getting emotional support or counseling for your loved one with dementia?

nn) Getting emotional support or counseling for you?

\section{FINDING OR ARRANGING FOR SERVICES}

oo) Knowing which service providers to ask for different types of help?

pp) Services offered by the County Area Agency on Aging/ state chapter of the Alzheimer's Association?

qq) Getting your loved one with dementia to accept help from service providers?

\begin{tabular}{|c|c|}
\hline NO & YES \\
\hline 0 & 1 \\
\hline 0 & 1 \\
\hline 0 & 1 \\
\hline 0 & 1 \\
\hline 0 & 1 \\
\hline 0 & 1 \\
\hline 0 & 1 \\
\hline 0 & 1 \\
\hline 0 & 1 \\
\hline 0 & 1 \\
\hline 0 & 1 \\
\hline 0 & 1 \\
\hline 0 & 1 \\
\hline 0 & 1 \\
\hline 0 & 1 \\
\hline 0 & 1 \\
\hline 0 & 1 \\
\hline 0 & 1 \\
\hline 0 & 1 \\
\hline 0 & 1 \\
\hline 0 & 1 \\
\hline
\end{tabular}


rr) Getting different service providers to work together?

ss) Finding services you or your loved one with dementia needs?

tt) Getting transportation to locations where services are provided? 1 
Benjamin Rose Institute Unmet Needs Assessment (Bass et al., 2012, 2013) Continued p. 5

Please read the list of topics. After reading each topic, I would like you to tell me if you or your loved one with Dementia need more information or help with this topic. Select No (0) or Yes (1).

\begin{tabular}{|l|c|c|}
\hline \multicolumn{1}{|c|}{$\begin{array}{c}\text { UNMET NEED ASSESSMENT - BENJAMIN ROSE INSTITUTE } \\
\text { ON AGING }\end{array}$} & $\begin{array}{l}\text { B. 17. Do you need more } \\
\text { information about or help with } \\
\text { thic? }\end{array}$ \\
\hline $\begin{array}{l}\text { LIVING ARRANGEMENTS } \\
\text { uu) Knowing the different places where your loved one with dementia } \\
\text { could live if he/she needed to move from his/her current home? }\end{array}$ & 0 & YES \\
\hline vv) Paying for assisted living or nursing home care? & 0 & 1 \\
\hline $\begin{array}{l}\text { ww) Getting information about assisted living facilities or nursing } \\
\text { homes? }\end{array}$ & 0 & 1 \\
\hline
\end{tabular}


Appendix N Variables by Research Questions with Measures Table 8

\section{PREDISPOSING}

What estimate of the variance in non-use of services in Appalachia can be explained by the predisposing characteristics of demographic, social, and belief variables?

\begin{tabular}{|c|c|}
\hline & Measures in study \\
\hline Demographic & $\begin{array}{l}\text { CG gender } \\
\text { CG age }\end{array}$ \\
\hline Social & $\begin{array}{l}\text { CG level of education } \\
\text { CG relationship to PWD } \\
\text { SPS } 10 \mathrm{R}\end{array}$ \\
\hline Health Beliefs (Dementia) & $\begin{array}{l}12 \text { Phillipson et al. (2013) questions about knowledge } \\
\text { dementia and treatment }\end{array}$ \\
\hline Health Beliefs Services (Dementia) & $\begin{array}{l}12 \text { different Phillipson et al, (2013) CG beliefs about } \\
\text { services- useful, not useful, harmful }\end{array}$ \\
\hline
\end{tabular}

\begin{tabular}{|c|c|}
\hline \multicolumn{2}{|c|}{$\begin{array}{l}\text { ENABLING } \\
\text { What estimate of the variance in the non-use of services in Appalachia can be explained by } \\
\text { enabling factors of financial and organizations resource variables? }\end{array}$} \\
\hline \multirow{2}{*}{\multicolumn{2}{|c|}{$\begin{array}{l}\text { What estimate of the variance in the non-use of services in Appalachia can be explained by } \\
\text { enabling factors of financial and organizations resource variables? }\end{array}$}} \\
\hline & \\
\hline & Measures in Study \\
\hline \multirow[t]{2}{*}{ Financial } & Household income \\
\hline & UNALF subscale total score \\
\hline \multirow[t]{2}{*}{ Organizing } & Total Caregiving Services Available (both F \& I) plus \\
\hline & total subscale scores UNAFC, UNAES, and UNAFS \\
\hline
\end{tabular}


UNA- Unmet Needs Assessment, Bass et al., 2012 LF-Legal Financial; FC-Family Concerns; ES-Emotional Support; FS-Finding Services

\begin{tabular}{|l|l|}
\hline \multicolumn{2}{|c|}{ NEED } \\
What estimate of the variance in non-use of services in Appalachia can be explained by the \\
\hline Evaluated & Combined variable: \\
\hline & PWD had HCP; \\
\hline Perceived & HCP recommended services; total weekly hours of \\
& formal and informal help received per week \\
\hline & \begin{tabular}{l} 
Combined variable: \\
Total score subscales UNAHI, UNADT, and UNALA; \\
PLUS \\
Total score Caregiver Mastery Subscale (Lawton et al., \\
\hline
\end{tabular} \\
\hline
\end{tabular}

UNA- Unmet Needs Assessment (Bass et al., 2012) HI-Health Information; DT- Daily living Tasks; LA- Living Arrangements

\begin{tabular}{|l|l|}
\hline \multicolumn{2}{|c|}{ Combined Variables from Three Research Questions } \\
What combination of predisposing, enabling, and need factors constitutes the strongest \\
explanatory or associative model of non-use of services? \\
\hline used) \\
\hline Predisposing & Strongest variable each question (Measure \\
\hline
\end{tabular}




\begin{tabular}{|l|l|}
\hline Enabling & $\begin{array}{l}\text { Total Caregiving Services Available (both } \\
\text { formal \& informal) }\end{array}$ \\
\hline Need & $\begin{array}{l}\text { Evaluated Need Combined variable: } \\
\text { PWD had HCP; } \\
\text { HCP recommended services; total weekly } \\
\text { hours of formal and informal help received } \\
\text { per week }\end{array}$ \\
\hline
\end{tabular}


Appendix O Table 9 Similarities and Differences

Combined Services, 8 Formal, 6 Informal Services and User/Non-User

\begin{tabular}{|c|c|c|c|c|c|}
\hline $\begin{array}{c}\text { Question and Non-Use of } \\
\text { Services }\end{array}$ & $\mathbf{N}$ & $\begin{array}{c}\text { Adjusted R } \\
\text { Square } \\
\end{array}$ & F Values & Sig. & $\begin{array}{l}\text { Most Robust } \\
\text { Explanatory }\end{array}$ \\
\hline $\begin{array}{l}\text { Q1 Combined (14 Both formal } \\
\text { (Gitlin) and Informal (CGS) }\end{array}$ & 40 & 0.083 & 4.550 & 0.039 & $\begin{array}{l}14 \text { Both, but } \\
\text { Formal DNL } \\
\text { separately }\end{array}$ \\
\hline Q1 8 Formal (Gitlin) & 40 & DNL & DNL & DNL & \\
\hline Q1 6 Informal (CGS) & 40 & 0.076 & 4.196 & 0.047 & \\
\hline \multicolumn{6}{|c|}{$\begin{array}{l}\text { Q1 Logistic Regression "User" } \mathrm{n}=17 \text { "Non-User" } \mathrm{n}=23 p=.048 \text { Odds Ratio is .728 (if CG } \\
\text { has higher SPS score, he/she is . } 728 \text { times as likely to have a higher Non-use of services; so } \\
\text { individuals who are higher in SPS are } 27 \% \text { less likely to be a non-user). }\end{array}$} \\
\hline $\begin{array}{l}\text { Q2 Combined (14 Both formal } \\
\text { (Gitlin) and Informal (CGS) }\end{array}$ & 38 & 0.117 & 5.894 & .020 & \\
\hline Q2 8 Formal (Gitlin) & 38 & DNL & DNL & DNL & \\
\hline Q2 6 Informal (CGS) & 38 & 0.137 & 6.878 & 0.013 & $\begin{array}{l}6 \text { Informal } \\
\text { Services Not } \\
\text { Used }\end{array}$ \\
\hline \multicolumn{6}{|c|}{ Q2 Logistic Regression "User" $\mathrm{n}=15$ "Non-User" $\mathrm{n}=23$ No significant variables. } \\
\hline $\begin{array}{l}\text { Q3 Combined (14 Both formal } \\
\text { (Gitlin) and Informal (CGS) }\end{array}$ & 42 & 0.162 & 2.456 & 0.078 & \\
\hline Q3 8 Formal (Gitlin) & 42 & 0.255 & 15.004 & 0.000 & $\begin{array}{l}8 \text { Formal } \\
\text { Services } \\
\text { Evaluated } \\
\quad \text { Need }\end{array}$ \\
\hline Q3 6 Informal (CGS) & 42 & DNL & DNL & DNL & \\
\hline \multicolumn{6}{|c|}{$\begin{array}{l}\text { Q3 Logistic Regression "User" } \mathrm{n}=16 \text { "Non-User" } \mathrm{n}=26 p=.029 \text { Odds Ratio is } .955 \text { (if CG } \\
\text { had hcp, hcp recommended services, \& CG had formal \& informal hours help per week, } \\
\text { he/she is } .955 \text { times as likely to have a higher Non-use of services; so individuals with higher } \\
\text { Evaluated Need are } 5 \% \text { less likely to be a non-user) }\end{array}$} \\
\hline & & & & & $\begin{array}{l}\text { Predictor and } \\
\text { Most Robust } \\
\text { Explanatory }\end{array}$ \\
\hline $\begin{array}{l}\text { Q4 Combined (14 Both formal } \\
\text { (Gitlin) and Informal (CGS) } \\
\text { Model } 2\end{array}$ & 42 & 0.353 & 12.197 & 0.002 & $\begin{array}{c}\text { Total } \\
\text { Caregiving } \\
\text { Services } \\
\text { Available .210 } \\
\ldots \ldots \ldots \ldots \ldots \ldots . . . . . . \\
\text { Evaluated } \\
\text { Need }-.041\end{array}$ \\
\hline Q4 8 Formal (Gitlin) & 42 & 0.259 & 15.350 & 0.000 & $\begin{array}{c}\text { Evaluated } \\
\text { Need }\end{array}$ \\
\hline
\end{tabular}




\begin{tabular}{|l|c|c|c|c|c|}
\hline Q4 6 Informal (CGS) & 42 & 0.169 & 9.364 & 0.004 & $\begin{array}{c}\text { Total CG } \\
\text { Services } \\
\text { Available }\end{array}$ \\
\hline
\end{tabular}

Q4 Logistic Regression "User" $\mathrm{n}=17$ "Non-User" $\mathrm{n}=25$ Evaluated Need $p=.011$ Odds Ratio is .935 (If CG has higher evaluated need [health care provider, who recommends services, using formal and informal services at home, he/she is .935 times as likely to have a higher Non-use of services; so individuals who are higher in SPS are 7.5\% less likely to be a non-user For Total Caregiving Services Available both formal and informal $p=.05$, Odds Ratio 1.251; So those who have higher total caregiving services, especially informal services available are $25 \%$ more likely to be a non-user.

Q1 is Research Question 1; Q2 is Research Question 2; Q3 is Research Question 3; Q4 is Research Question 4 DNL- variable did not load into equation; Evaluated Need with having HCP, HCP recommended services, and paid hours help with volunteer hours help per week; Total CG Services Available Both Formal and Informal 
Appendix P Family Caregiver Supports Included with Survey Packet

Please complete survey first, then contact any agency below that you do not already use.

The following resources can help guide family caregivers of persons with dementia and serve as a place to start getting support. Each phone number is for the National organization, which can provide your local contact information.

\begin{tabular}{|c|c|c|c|}
\hline $\begin{array}{l}\text { National } \\
\text { Organization }\end{array}$ & Contact Information & Brief Description & $\begin{array}{l}\text { Suggested } \\
\text { Questions }\end{array}$ \\
\hline $\begin{array}{l}\text { Alzheimer's } \\
\text { Association }\end{array}$ & $\begin{array}{l}\text { 24/7 Toll-Free Helpline } \\
\mathbf{8 0 0 - 2 7 2 - 3 9 0 0} \\
\text { www.alz.org } \\
\text { Caregiver Center is } \\
\text { http://www.alz.org/care/ }\end{array}$ & $\begin{array}{l}\text { Provide education } \\
\& \text { support service to } \\
\text { individuals with } \\
\text { Dementias, families, } \\
\text { and Caregivers }\end{array}$ & $\begin{array}{l}\text { Request the } \\
\text { Alzheimer's } \\
\text { Association } \\
\text { Caregiver } \\
\text { Handbook }\end{array}$ \\
\hline $\begin{array}{l}\text { Administration } \\
\text { on Aging } \\
\text { (AoA) }\end{array}$ & $\begin{array}{l}\text { www.aoa.gov } \\
\text { Eldercare Locator (to } \\
\text { find local resources): } \\
\text { (800) 677-1116 }\end{array}$ & $\begin{array}{l}\text { Federal agency } \\
\text { helping with } \\
\text { information for } \\
\text { older people and } \\
\text { caregivers }\end{array}$ & $\begin{array}{l}\text { Supportive } \\
\text { Services and } \\
\text { Senior Centers } \\
\text { Program }\end{array}$ \\
\hline $\begin{array}{l}\text { Family } \\
\text { Caregiver } \\
\text { Alliance }\end{array}$ & $\begin{array}{l}\text { 1-800-445-8106 } \\
\text { www.caregiver.org } \\
\end{array}$ & $\begin{array}{l}\text { Resources for } \\
\text { family caregivers, } \\
\text { like Family Care } \\
\text { Navigator, a state- } \\
\text { by-state list of } \\
\text { services }\end{array}$ & $\begin{array}{l}\square \text { Caregiver Facts } \\
\text { and Tips Sheets } \\
\text { The website } \\
\text { has links to } \\
\text { videos and } \\
\text { webinars }\end{array}$ \\
\hline $\begin{array}{l}\text { AARP } \\
\text { Caregiver } \\
\text { Hotline }\end{array}$ & $\begin{array}{l}\text { 1-877-333-5885 Mon.- } \\
\text { Fri. 7am-11pm EST, Sat. } \\
\text { 9am-5pm } \\
\text { www.aarp.org/caregiving }\end{array}$ & $\begin{array}{l}\text { Connects members } \\
\text { with local resources } \\
\text { to service agencies } \\
\text { in their area }\end{array}$ & $\begin{array}{l}\text { Ask about } \\
\text { Prepare to } \\
\text { Care Guide }\end{array}$ \\
\hline
\end{tabular}

The above list is to help you get started. The guidebooks and websites have links or contact information for many other support agencies and services. Perhaps try one agency per week or request the Caregiver Guides (Caregiver Handbook and Prepare to Care Guide) and begin reading.

Please see the back of this page for further information. 


\section{Other caregivers of persons with dementia report it is helpful to:}

Eat Healthy, Exercise, and Get enough Sleep: In order to help others, you need to be your best self. All three of these steps help you manage stress and protect your own health and safety.

Ask for Help: This may be hard to do, but having support of family members, friends, church members, and neighbors can help you keep the person with dementia as safe and healthy as you can. Sharing in care can be asking someone to call or visit at a certain time of day, help drive the person with dementia to an appointment, shopping for or making food, etc. The person with dementia's health care team can work with you, as well as support groups, such as the Alzheimer's Association. Using supports such as home health, meal delivery, adult day care, and respite care help keep both caregivers and persons with dementia safe and as healthy as possible.

Stay organized: Use calendars, medication schedules and lists to keep track of the person with dementia. Keep lists in one notebook or folder with health care information (medications, medical history, and health care provider phone numbers) and insurance information. [Share the information with all caregivers helping the person with dementia.] Keep a list of support services in the folder/notebook.

Plan for future care: Living Wills, Medical Power of Attorney, Transportation and Driving Safety, End-of-life decisions, financial matters, Legal issues, Safety of the home (fire, fall prevention, firearms, cooking, etc.) The guidebooks listed on the front page give you details about each issue.

Knowledge about dementia is a way to give the best care. Many books, websites, and services (like those listed on the front of this paper) have medical information about dementia, the stages of dementia, what to expect, how to handle certain actions and challenges, working with family (as well as friends, neighbors, etc.), and other parts of caregiving for a person with dementia. 
Appendix Q Revised Scales and Subscale Internal Consistencies and Reliabilities

Revised Unmet Needs Assessment Benjamin Rose Institute (Bass et al., 2012; 2013)

\begin{tabular}{|c|c|c|c|c|c|c|}
\hline \multicolumn{7}{|c|}{ Bass et al. (2013) Benjamin Rose Unmet Needs Data } \\
\hline Variable name & $\begin{array}{l}\text { Possible } \\
\text { range }\end{array}$ & $\begin{array}{l}\text { Actual } \\
\text { range }\end{array}$ & $\begin{array}{l}\text { Mean } \\
(\mathrm{SD})\end{array}$ & Median & Mode & Literature reported values \\
\hline $\begin{array}{l}\text { FAMILY } \\
\text { CONCERNS }\end{array}$ & $0-7$ & $0-7$ & 1.76 & 1.00 & 0 & \\
\hline $\begin{array}{l}\text { HEALTH } \\
\text { INFORMATION }\end{array}$ & $0-15$ & $0-15$ & 6.07 & 6.50 & 0 & \\
\hline $\begin{array}{l}\text { DAILY LIVING } \\
\text { TASKS }\end{array}$ & $0-5$ & $0-5$ & 1.6 & 1.00 & 0 & \\
\hline $\begin{array}{l}\text { RESPITE } \\
\text { SERVICES }\end{array}$ & $0-2$ & $0-2$ & 1.02 & 1.00 & 2 & \\
\hline $\begin{array}{l}\text { LEGAL AND } \\
\text { FINANCIAL } \\
\text { ISSUES }\end{array}$ & $0-7$ & $0-7$ & 2.12 & 2.00 & 0 & \\
\hline $\begin{array}{l}\text { EMOTIONAL } \\
\text { SUPPORT OR } \\
\text { COUNSELING }\end{array}$ & $0-4$ & $0-4$ & 1.45 & 1.00 & 0 & \\
\hline $\begin{array}{l}\text { FINDING OR } \\
\text { ARRANGING FOR } \\
\text { SERVICES }\end{array}$ & $0-6$ & $0-5$ & 2.02 & 2.00 & 1 & \\
\hline $\begin{array}{l}\text { LIVING } \\
\text { ARRANGEMENTS }\end{array}$ & $0-3$ & $0-3$ & 1.62 & 2.00 & 3 & \\
\hline $\begin{array}{l}\text { Total UNMET } \\
\text { NEEDS } \\
\text { ASSESSMENT } \\
\text { scale }\end{array}$ & $0-49$ & $0-40$ & $\begin{array}{l}17.67 \\
(12.291)\end{array}$ & 19.00 & 0 & $\begin{array}{l}\text { Range } 0-39 \text {, Mean 17.04, SD } 11.8 \\
\text { for } 39 \text { question scale (Bass et al., } \\
2012 \text {, p.243) } \\
\text { Unmet Need High 6-month } \\
\text { comparison (18.2) v intervention } \\
\text { group (18.1) } \\
\text { 12-month comparison (14.2) v } \\
\text { Intervention group (11.1). Mean } \\
\text { change - } 4.0 \text { comparison group v } \\
6.4 \text { intervention group (Bass et al., } \\
2013 \text {, p. 1384) } \\
\text { Bass et al. (2014) Baseline Mean } \\
6.24 \text { (SD 6.56). }\end{array}$ \\
\hline
\end{tabular}


Phillipson, Magee, \& Jones (2013) Beliefs Health Services and Service Beliefs

\begin{tabular}{|c|c|c|c|c|c|}
\hline $\begin{array}{l}\text { Variable } \\
\text { Name(s) }\end{array}$ & $\begin{array}{l}\text { Possible } \\
\text { Range }\end{array}$ & $\begin{array}{l}\text { Actual } \\
\text { Range }\end{array}$ & Mean & $\begin{array}{l}\text { Standard } \\
\text { Deviation }\end{array}$ & $\begin{array}{l}\text { Literature } \\
\text { Reported Values }\end{array}$ \\
\hline $\begin{array}{l}\text { 11 Health } \\
\text { Belief } \\
\text { Statements (4 } \\
\text { about } \\
\text { dementia } \\
\text { stigma, } 3 \\
\text { from } \\
\text { qualitative } \\
\text { study from } \\
\text { above } \\
\text { authors, } 4 \\
\text { beliefs about } \\
\text { effectiveness } \\
\text { of treatment } \\
\text { for dementia } \\
\text { (Bond et al., } \\
\text { 2005) }\end{array}$ & $\begin{array}{l}7-56 \\
\text { Behavioral } \\
4-16 \\
\text { Control } \\
1-4 \\
\text { Access } \\
4-16 \\
\text { Suitability } \\
\text { 1-4 } \\
\text { Normative } \\
\text { 3-12 } \\
\text { Tot. Family } \\
\text { 1-4 } \\
\text { Govt. } \\
\text { 4-8 }\end{array}$ & $\begin{array}{l}10-38 \\
\text { Behavioral } \\
2-12 \\
\text { Control } \\
1-4 \\
\text { Access } \\
1-13 \\
\text { Suitability } \\
1-3 \\
\text { Normative } \\
3-12 \\
\text { Tot.Family } \\
\text { 1-4 } \\
\text { Govt. } \\
\text { 4-8 }\end{array}$ & $\begin{array}{l}28.47 \text { total } \\
\text { Behavioral } \\
8.60 \\
\text { Control } \\
2.89 \\
\text { Access } \\
8.28 \\
\text { Suitability } \\
1.97 \\
\text { Normative } \\
9.95 \\
\text { Tot.Family } \\
2.91 \\
\text { Govt. } \\
8.0\end{array}$ & $\begin{array}{l}5.457 \text { total } \\
\text { Behavioral } \\
2.548 \\
\text { Control } \\
1.022 \\
\text { Access } \\
3.089 \\
\text { Suitability } \\
0.545 \\
\text { Normative } \\
1.713 \\
\text { Tot. Family } \\
0.840 \\
\text { Govt. } \\
1.048\end{array}$ & $\begin{array}{l}\text { Phillipson et al., } \\
(2013) \text { reported } \\
\text { Service Beliefs as } \\
\text { mean }=49 \text { for } \\
\text { Behavioral } \\
\text { Beliefs } \mathrm{SD}= \\
(43.4) \\
\text { Control mean }= \\
46, \mathrm{SD}=(40.7) \\
\text { Access mean }=48 \text {, } \\
\mathrm{SD}=(42.5) \\
\text { Suitability mean= } \\
21, \mathrm{SD}=(18.6) \\
\text { Normative (needs } \\
\text { as defined by } \\
\text { professionals }) \\
\text { reported as total } \\
\text { family beliefs } \\
\text { mean }=6.78, \text { and } \\
\mathrm{SD}=(1.18) \text { and }\end{array}$ \\
\hline
\end{tabular}




\begin{tabular}{|l|l|l|l|l|}
\hline & & & & $\begin{array}{l}\text { total government } \\
\text { beliefs mean= } \\
6.93, \mathrm{SD}=(1.04)\end{array}$ \\
\hline
\end{tabular}

Phillipson, L., Magee, C., and Jones, S.C. (2013). Why carers of people with dementia do no utilize out-of-home respite services. Health and Social Care in the Community, 21(4), 411-422. Doi: $10.1111 /$ hsc. 12030

Normative Beliefs in table with two scores for total family and total government- ran freq. with descriptives both ways. 
Social Provisions Scale (Russell \& Cutrona, 1984 )

\begin{tabular}{|l|l|l|l|l|l|}
\hline $\begin{array}{l}\text { Variable } \\
\text { Name(s) }\end{array}$ & $\begin{array}{l}\text { Possible } \\
\text { Range }\end{array}$ & $\begin{array}{l}\text { Actual } \\
\text { Range }\end{array}$ & Mean & $\begin{array}{l}\text { Standard } \\
\text { Deviation }\end{array}$ & $\begin{array}{l}\text { Literature Reported } \\
\text { Values }\end{array}$ \\
\hline $\begin{array}{l}\text { Total SPS } \\
\text { Scale }\end{array}$ & $10-40$ & $16-37$ & 30.42 & 3.935 & $\begin{array}{l}\text { Hoven (2012) mean } \\
34.54 \text { (SD is 4.66) }\end{array}$ \\
& & & & $\begin{array}{l}\text { Iapichino et al., (2016) } \\
\text { mean 35.13 (SD 4.4). }\end{array}$ \\
\hline
\end{tabular}


Appendix R Focused Content Analysis Respondent Comments $(\mathrm{n}=24)$

\begin{tabular}{|c|c|c|}
\hline Topics & $\mathbf{n}=$ & $\begin{array}{c}\text { Literature Reporting Comparable } \\
\text { Findings } \\
\end{array}$ \\
\hline $\begin{array}{l}\text { "Don't need additional services/don't need } \\
\text { services yet" }\end{array}$ & 4 & $\begin{array}{l}\text { Do not use because PWD doesn't } \\
\text { need a lot of assistance with ADLs, } \\
\text { IADLs, or already using service that } \\
\text { meets current needs (Brodaty et al., } \\
\text { 2005; Phillipson et al., 2013). }\end{array}$ \\
\hline "Not Qualifying/Not eligible for services" & 7 & $\begin{array}{l}\text { To qualify for some caregiving } \\
\text { support resources, the PWD must be } \\
\text { eligible for Medicare and/or } \\
\text { Medicaid (Foldes, Moriarty, Farseth, } \\
\text { Mittelman, \& Hall Long, 2018; } \\
\text { Morhardt, 2011; Tremont et al., } \\
\text { 2017). Brown, Friedemann, \& } \\
\text { Mauro (2014) note some adult day } \\
\text { services will offer sliding scale } \\
\text { payment if not able to afford, } \\
\text { because32 do not qualify for other } \\
\text { services. }\end{array}$ \\
\hline "Lack of Knowledge of existing services" & 3 & $\begin{array}{l}\text { Neville, Beattie, Fielding, \& } \\
\text { MacAndrew (2015) reported a } \\
\text { significant contributer to non-use of } \\
\text { respite services includes lack of } \\
\text { knowledge by CGs of availability of } \\
\text { the services. (p. 53) } \\
\text { Mast (2013) identified key factors } \\
\text { about services are marketing and } \\
\text { information for families. } \\
\text { A significant barrier to service use } \\
\text { was lack of knowledge about } \\
\text { dementia services, including } \\
\text { dementia dedicated services and } \\
\text { social services (Parveen et al., 2017; } \\
\text { Phillipson et al., 2013; Singh, } \\
\text { Hussain, Khan, Irwin, \& Foskey, } \\
\text { 2014) }\end{array}$ \\
\hline "No stranger in home" & 5 & $\begin{array}{l}\text { Nothing specific in literature about } \\
\text { "stranger in home", but CG wanting } \\
\text { continuity of staff or personnel } \\
\text { Górska et al. (2013). Researchers } \\
\text { described CG who had consistent } \\
\text { support personnel reported enjoying } \\
\text { interaction with same paid service, } \\
\text { but CG who experienced }\end{array}$ \\
\hline
\end{tabular}




\begin{tabular}{|c|c|c|}
\hline Topics & $\mathbf{n}=$ & $\begin{array}{c}\text { Literature Reporting Comparable } \\
\text { Findings }\end{array}$ \\
\hline & & $\begin{array}{l}\text { inconsistent staff reported increased } \\
\text { distress (Górska et al., 2013). } \\
\text { Macleod et al. (2017) listed CG may } \\
\text { not use services because of negative } \\
\text { experience with services or not } \\
\text { wanting a "stranger" in the home. } \\
\text { O'Connell et al. (2012) also reported } \\
\text { CGs wanted stability and continuity } \\
\text { of staff, requesting commitment } \\
\text { from staff to role as carers. }\end{array}$ \\
\hline "Too much information too soon (overwhelmed) & 1 & $\begin{array}{l}\text { CGs identified being overwhelmed } \\
\text { with too much information (Brodaty } \\
\text { \& Donkin, 2009; McCabe, You, \& } \\
\text { Tatangelo, 2016; Neville et al., } \\
\text { 2015; and Robinson et al., 2012) }\end{array}$ \\
\hline "Needs help accessing resources" & 1 & $\begin{array}{l}\text { Mast (2013) described family help } \\
\text { seeking patterns as an important } \\
\text { experiential factor influencing the } \\
\text { use or non-use of services. } \\
\text { Macleod et al. (2017) reported one } \\
\text { of six barriers to service use as } \\
\text { difficulty finding information about } \\
\text { relevant services or support. }\end{array}$ \\
\hline $\begin{array}{l}\text { Opposition/Struggle between CG/PWD about } \\
\text { need/refusal of services (Hard for PWD to } \\
\text { accept help or PWD may deny need for help) }\end{array}$ & 4 & $\begin{array}{l}\text { Research indicates the PWD may } \\
\text { refuse support services (Robinson et } \\
\text { al., 2012; Takai, Yamamoto-Mitani, } \\
\text { Okamoto, Fukahori, \& Tanaka, } \\
\text { 2013; Tremont et al., 2017). }\end{array}$ \\
\hline $\begin{array}{l}\text { "Wanting permission to seek more } \\
\text { resources/support help" }\end{array}$ & 4 & $\begin{array}{l}\text { Neville et al. (2015) identified three } \\
\text { steps CG needs to do to accept } \\
\text { respite services: recognize need to } \\
\text { step away from CG role; giving } \\
\text { themselves permission to leave role } \\
\text { temporarily; and having available } \\
\text { social support services to take a } \\
\text { break from the CG role. } \\
\text { CG may have opposed to giving } \\
\text { permission to self to use respite care } \\
\text { because of guilt from deserting } \\
\text { PWD, not meeting marital or family } \\
\text { duty, or effect on PWD behavior or } \\
\text { emotions (Neville et al., 2015). } \\
\text { "Permission" was not specific in the } \\
\text { caregiving literature, perhaps "self- }\end{array}$ \\
\hline
\end{tabular}




\begin{tabular}{|c|c|c|}
\hline Topics & $\mathbf{n}=$ & $\begin{array}{c}\text { Literature Reporting Comparable } \\
\text { Findings }\end{array}$ \\
\hline & & $\begin{array}{l}\text { care" or "empowerment" may be } \\
\text { similar, but this needs further study } \\
\text { (Coon et al., 2016; Whitlatch \& } \\
\text { Orsulic-Jeras, 2018) }\end{array}$ \\
\hline "Navigation" & 1 & $\begin{array}{l}\text { Mast (2013) reported coordination } \\
\text { of services as a factor influencing } \\
\text { service use } \\
\text { Macleod et al. (2017) specified most } \\
\text { CGs wanted a central point of } \\
\text { contact, many times a case-manager } \\
\text { was the most beneficial, to act as an } \\
\text { entry to services. } \\
\text { Reuben et al. (2013) report low } \\
\text { utilization of services because of } \\
\text { community based care being poorly } \\
\text { integrated into the health care } \\
\text { system, deficit of knowledge about } \\
\text { dementia and dementia services, as } \\
\text { well as deficient coordinating due to } \\
\text { lack of time by health care } \\
\text { providers. The researchers } \\
\text { recommended using a nurse } \\
\text { practitioner as a Dementia Care } \\
\text { Manager in a co-management model } \\
\text { to coordinate and liaise with health } \\
\text { care providers, families, and } \\
\text { community-based organizations } \\
\text { (Reuben et al., 2013). }\end{array}$ \\
\hline "Need for financial resources" & 1 & $\begin{array}{l}\text { Mast (2013) included cost and } \\
\text { affordability as a factor affecting } \\
\text { service use. Both Coon et al. (2016) } \\
\text { and (World Health Organization and } \\
\text { Alzheimer's Disease International } \\
(2015)\end{array}$ \\
\hline "Feeling family obligation to provide care" & 1 & $\begin{array}{l}\text { MacLeod et al. (2017) described one } \\
\text { of six barriers to service use as CG } \\
\text { beliefs about responsibility of the } \\
\text { caregiving role (duty or obligation). } \\
\text { Phillipson et al., (2014) described } \\
\text { CG with sense of duty or obligation } \\
\text { to provide care tended to not use } \\
\text { services. } \\
\text { Mast (2013) identified relationship } \\
\text { factor encouraging service use as the }\end{array}$ \\
\hline
\end{tabular}




\begin{tabular}{|l|l|l|}
\hline \multicolumn{1}{|c|}{ Topics } & $\mathbf{n}=$ & $\begin{array}{l}\text { Literature Reporting Comparable } \\
\text { Findings }\end{array}$ \\
\hline & & $\begin{array}{l}\text { dynamics of CG relationship to } \\
\text { PWD (obligation, culpability, } \\
\text { betrayal). }\end{array}$ \\
\hline $\begin{array}{l}\text { "CG lacks trust someone can do as well as } \\
\text { he/she can" }\end{array}$ & 1 & $\begin{array}{l}\text { MacLeod et al. (2017) included a } \\
\text { mistrust of services as a barrier to } \\
\text { service use. } \\
\text { Orpin et al. (2014) stated CGs were } \\
\text { averse to give the responsibility of } \\
\text { PWD care to others, had to have a } \\
\text { lot of trust to share responsibility, } \\
\text { and frequently used professional } \\
\text { providers. }\end{array}$ \\
\hline
\end{tabular}

